\title{
Structure and Mechanism of the Flavoenzyme Lipoamide Dehydrogenase from Escherichia coli
}

\author{
Dissertation \\ for the award of the degree \\ "Doctor rerum naturalium" \\ of the Georg-August-Universität Göttingen
}

within the doctoral program Biomolecules: Structure-Function-Dynamics of the Georg-August-University School of Science (GAUSS)

submitted by

Michael Tietzel

from Holzminden

Göttingen 2015 
Members of the Thesis Committee

Prof. Dr. Kai Tittmann (Reviewer)

Department of Molecular Enzymology

Georg-August-Universität Göttingen

Prof. Dr. Ralf Ficner (Reviewer)

Department of Molecular Structural Biology

Georg-August-Universität Göttingen

Prof. Dr. Marina Rodnina

Department of Physical Biochemistry

Max Planck Institute for Biophysical Chemistry Göttingen

\section{Further members of the Examination Board}

Dr. Fabian Commichau

Jun.-Prof. Dr. Ricardo Mata

Dr. Manfred Konrad
Department of General Microbiology Georg-August-Universität Göttingen

Institute of Physical Chemistry Georg-August-Universität Göttingen

Enzyme Biochemistry Max Planck Institute for Biophysical Chemistry Göttingen

Date of oral examination: 10.09.2015 
Herewith I declare that I prepared this thesis "Structure and Mechanism of the Flavoenzyme Lipoamide Dehydrogenase from Escherichia coli" independently and with no other sources and aids than quoted. This thesis (wholly or in part) has not been submitted elsewhere for any academic award or qualification.

Göttingen, 31.07.2015

Michael Tietzel 


\section{Table of Contents}

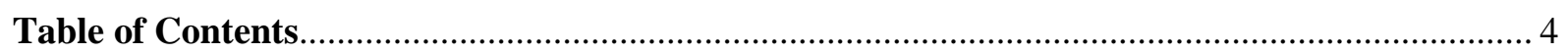

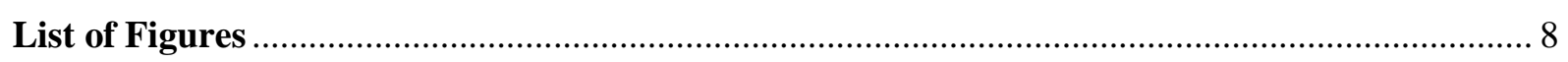

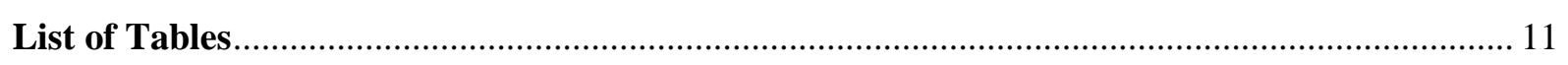

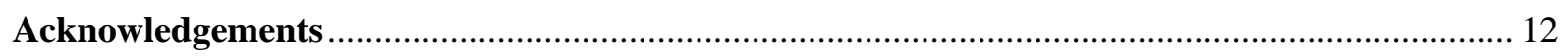

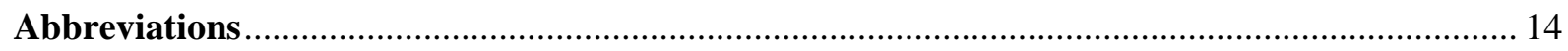

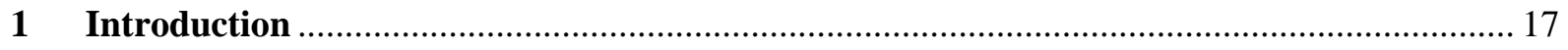

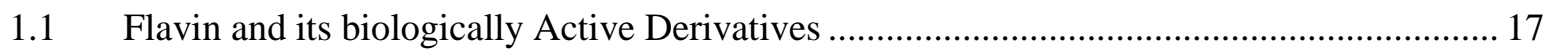

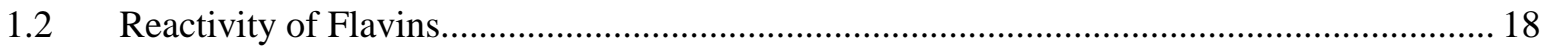

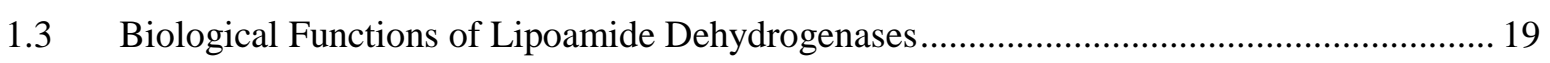

1.4 Three Dimensional Structure of E. coli Lipoamide Dehydrogenase .................................... 21

1.5 Catalytic Mechanism of E. coli Lipoamide Dehydrogenase ................................................ 23

1.6 The Physiological Importance of E. coli Lipoamide Dehydrogenase ................................... 25

1.7 The Relevance of Covalent Flavin-C4a-cysteinyl Adducts................................................ 28

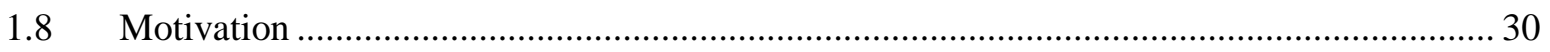

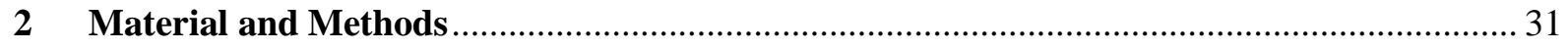

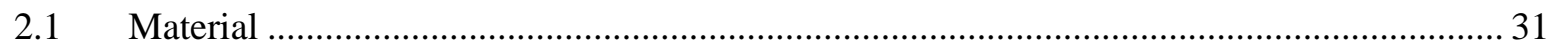

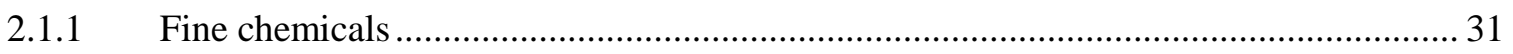

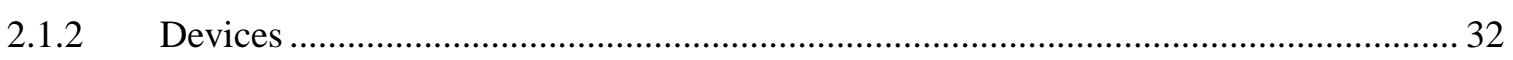

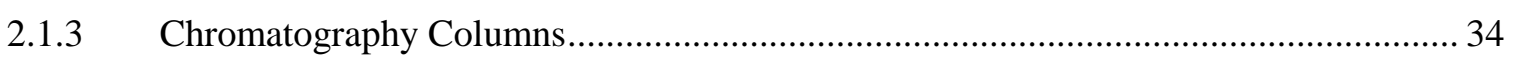

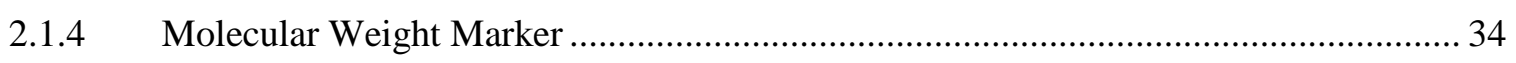

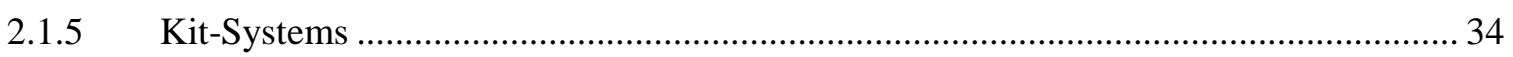

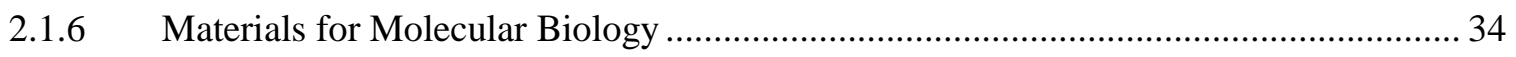

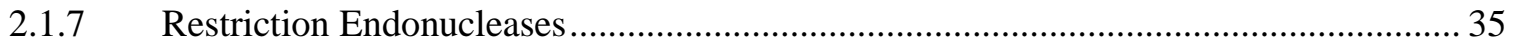

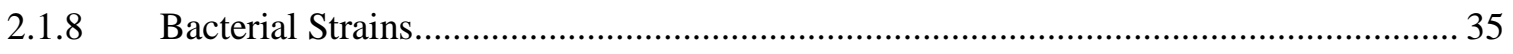

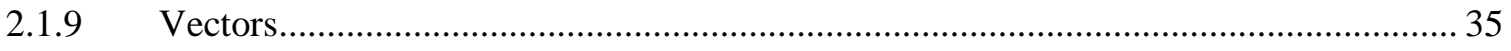

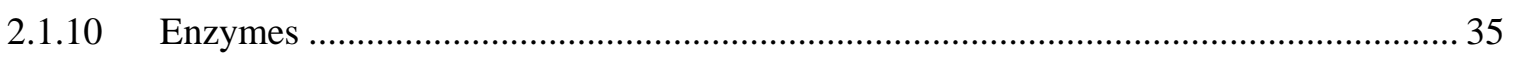

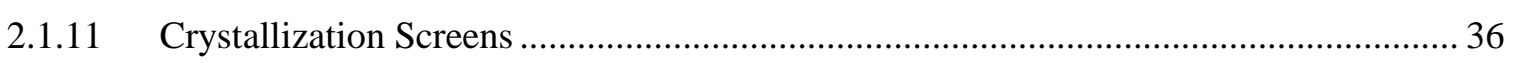

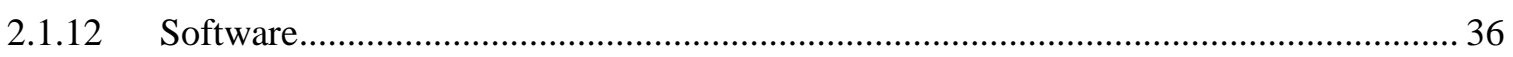

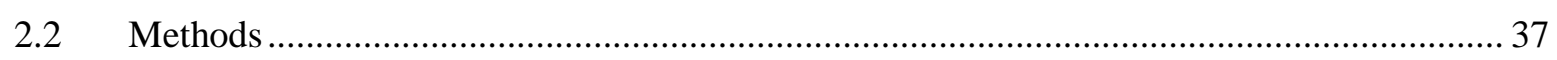

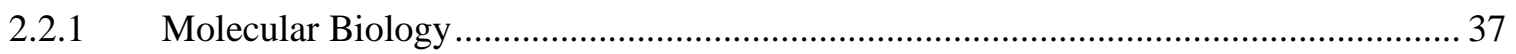

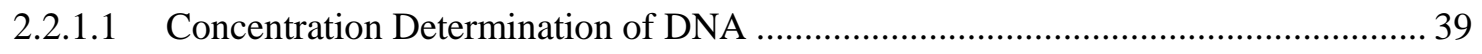

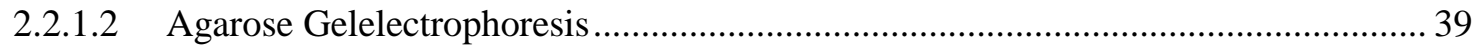

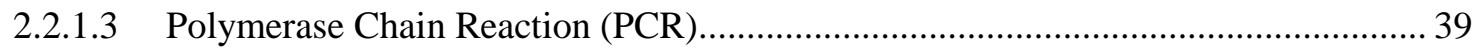

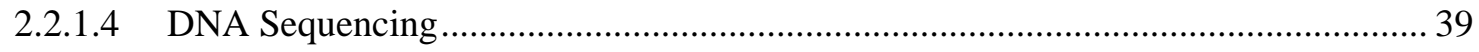




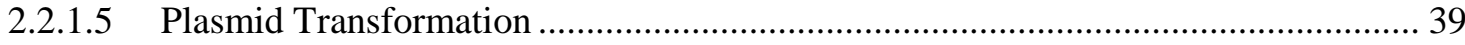

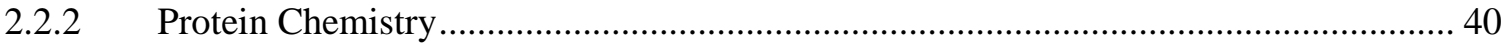

2.2.2.1 Sodium Dodecyl Sulfate - Polyacrylamide Gel Electrophoresis (SDS-PAGE) ........ 40

2.2.2.2 Protein Expression and Purification of EcE3 and multiple Variants from pGS523.. 40

2.2.2.3 Protein Expression and Purification of EcE3 and multiple Variants from pET28a .. 41

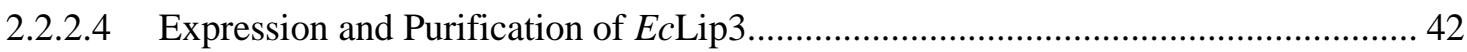

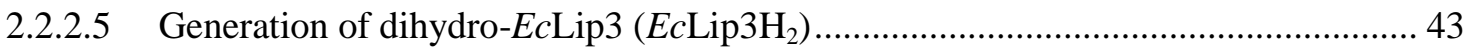

2.2.2.6 Concentration Determination of Proteins ................................................................... 43

2.2.2.7 Concentration Determination of dihydro-EcLip3 …............................................... 43

2.2.2.8 Determination of Extinction Coefficients of Multiple Variants .................................. 44

2.2.2.9 Determination of Redox Potentials of selected $E c E 3$ variants ................................ 45

2.2.2.10 Photobleaching of $E c E 3$ and Variants thereof................................................. 45

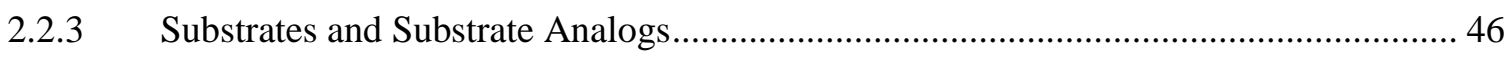

2.2.3.1 Concentration Determination of Substrates and Cofactors ........................................ 46

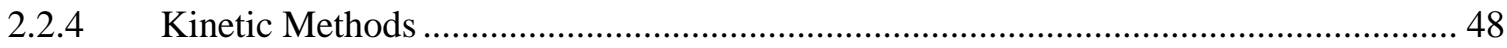

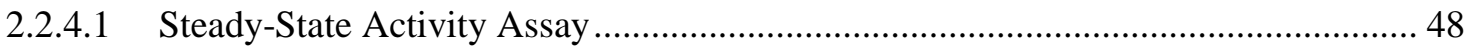

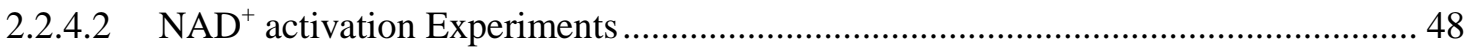

2.2.4.3 pH Dependency Experiments under a constant ionic Strength ................................. 48

2.2.4.4 Fast Kinetics-Stopped Flow Absorbance Spectroscopy ............................................ 48

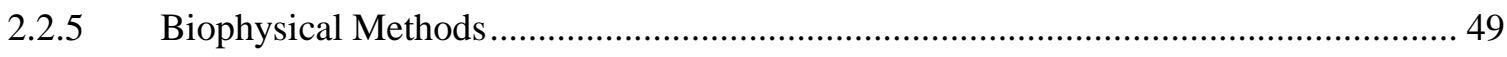

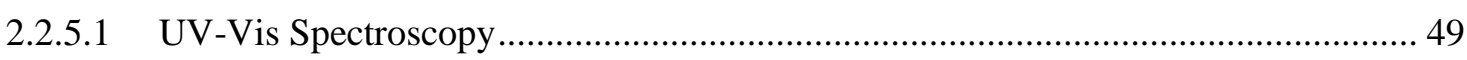

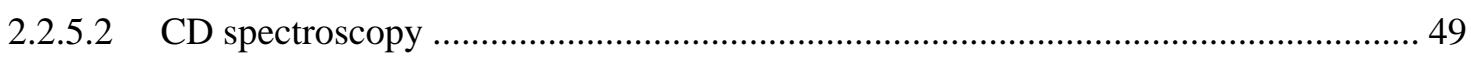

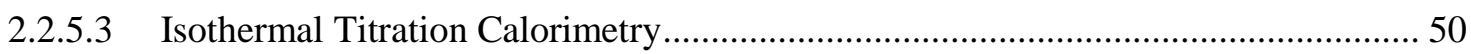

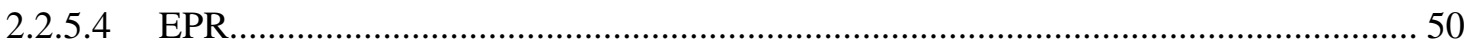

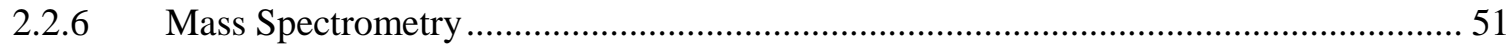

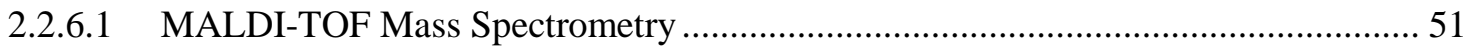

2.2.6.2 Determination of the Lipoylation State of EcLip3 .................................................. 51

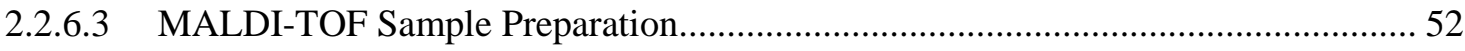

2.2.6.4 MALDI-TOF Measurement and Data Evaluation...................................................... 52

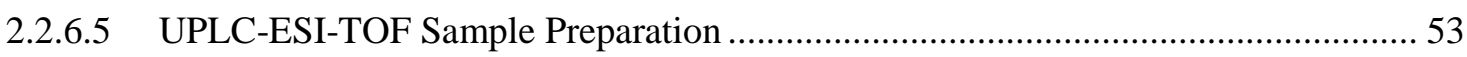

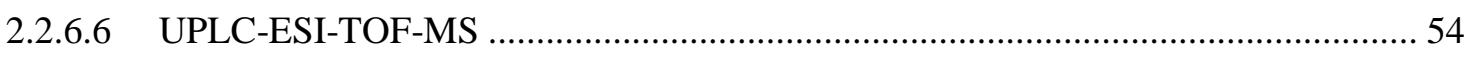

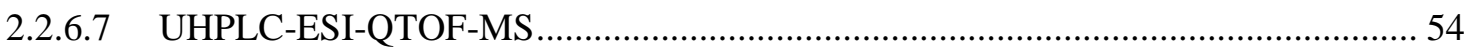

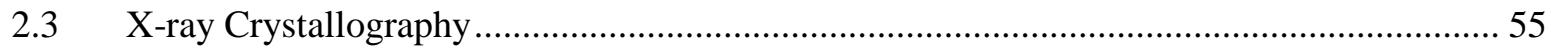

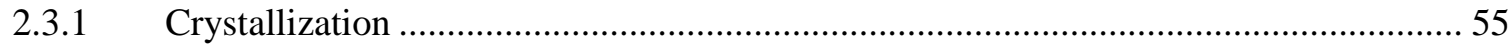

2.3.2 Data Processing and Molecular Replacement .............................................................. 56

2.3.3 Model Building, Refinement and Validation .............................................................. 56

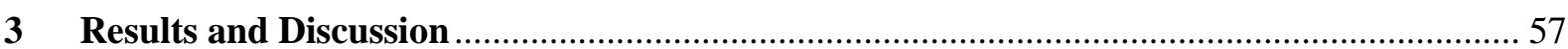




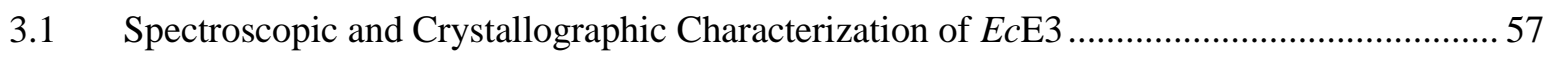

3.1.1 Kinetic Characterization of EcE3 in dependence of Lipoamide, $\mathrm{NAD}^{+}$and $\mathrm{pH}$............57

3.1.2 Detection of Reaction Intermediates using Stopped-flow Absorbance Spectroscopy... 60

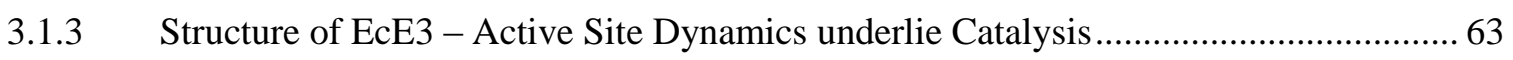

3.1.4 Spectroscopic and Crystallographic Investigation of the two-electron reduced State -

$\mathrm{EH}_{2}$

3.1.5 Spectral Evidence for the Cysteinyl-C4a Adduct in a monothiol variant of $E c E 3$........ 69

3.1.6 Crystallographic detection of the Cysteinyl-C4a Adduct.............................................. 72

3.1.7 Is the geometry of the FAD-C4a-cysteinyl adduct the fundament for its transience? ... 75

3.2 Investigation of the reductive half-reaction of $E c E 3$ using $E c L i p 3$ as native Substrate........ 77

3.2.1 Characterization of $E c \mathrm{Lip} 3$ and its Reduction to the dihydro-form $\left(E c \mathrm{Lip} 3 \mathrm{H}_{2}\right) \ldots \ldots \ldots . . .78$

3.2.2 $E c{\mathrm{Lip} 3 \mathrm{H}_{2}}_{2}$ as native Substrate to track the reductive Half-reaction of $E c \mathrm{E} 3$................. 81

3.2.3 Evidence for the Transience of the Cysteinyl-C4a Adduct .......................................... 83

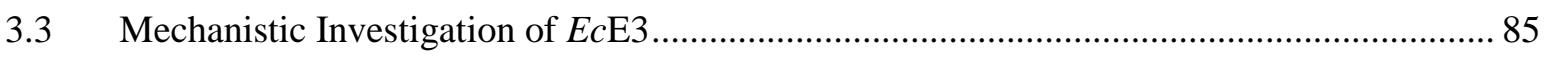

3.3.1 Characterization of the Reductive Half-Reaction using selected Active Site Variants. 85

3.3.1.1 Histidine445 and Glutamate450 - The Role of the Catalytic Diad during Catalysis 87

3.3.1.2 Tyrosine19 acts as structural Element rather than as a catalytic Residue ................. 90

3.3.2 Characterization of the Oxidative Half-reaction using selected Active Site Variants... 91

3.3.2.1 Lys54 and Glu189 are crucial Mediators of the Flavin Redox Potential.................... 97

3.3.2.2 A direct Involvement of Arg386 in a Proton Transfer Reaction is questionable ...... 97

3.4 Converting $E c E 3$ into a light-sensitive blue Light Receptor ............................................. 99

3.4.1 $E c E 3 C 45 \mathrm{~A} / \mathrm{K} 54 \mathrm{Q}$ and C45S/K54Q - PseudoLOV variants ..................................... 99

3.4.2 Photobleaching of pseudoLOV Variants leads to irreversible Alterations at the Flavin

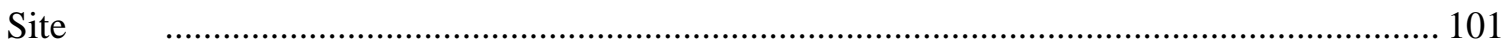

3.4.3 Mass Spectrometric Analysis of the photobleached Flavin Cofactor ........................... 104

3.4.3.1 C4a-cysteinyl Adduct Formation in LOV Domains prevents Photodegradation of the

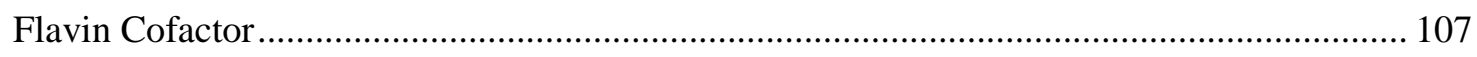

3.4.3.2 Photodegradation of enzyme-bound FAD leads to Double Bond formation in the ribityl part.

3.4.4 The H-bonding Network around the Flavin - The next Step on the Way to a light-

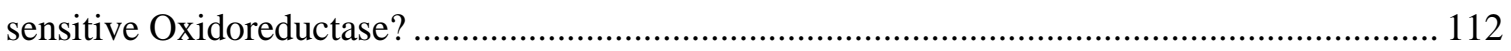

3.5 Expanding the Active Site - Glu354 is also a Mediator of the Flavin Redox Potential...... 114

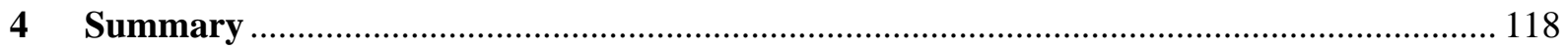

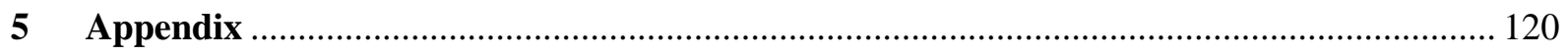

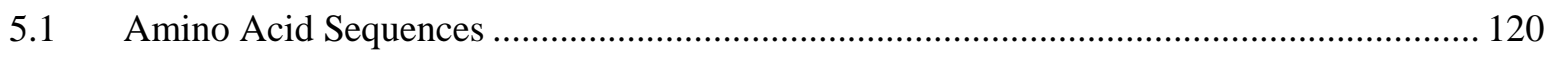

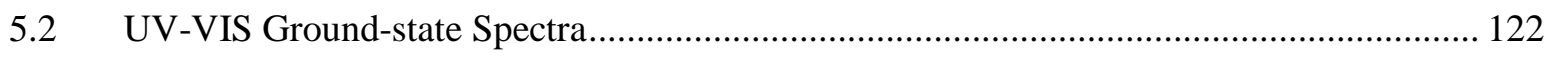




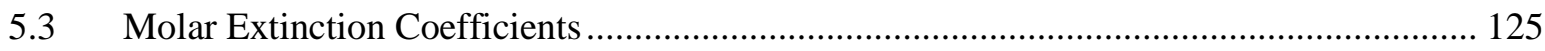

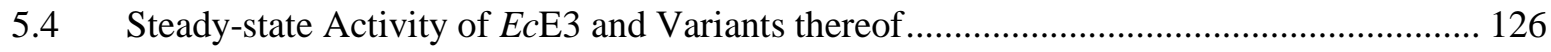

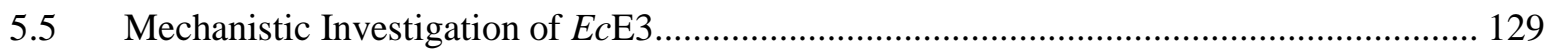

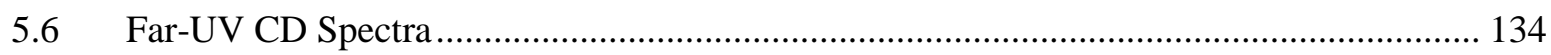

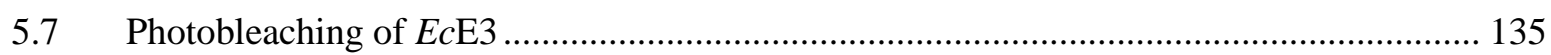

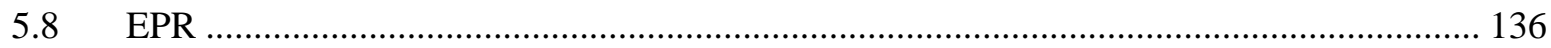

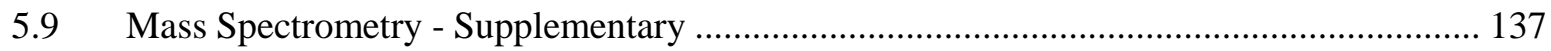

5.10 Redox Potentials of selected EcE3 Variants ................................................................... 147

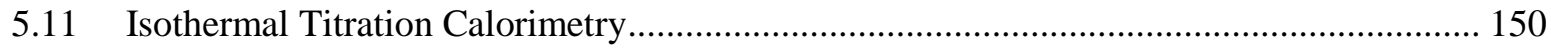

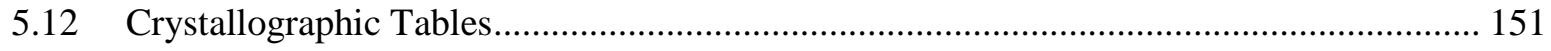

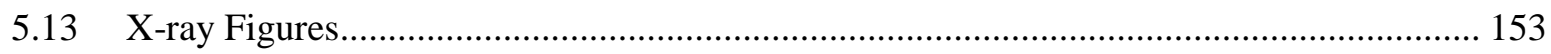

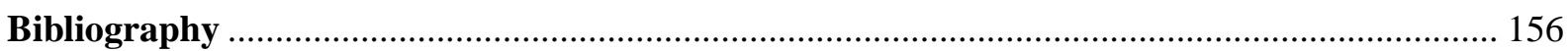

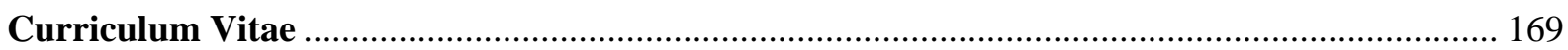




\section{List of Figures}

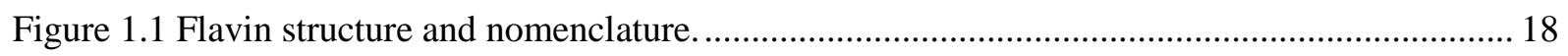

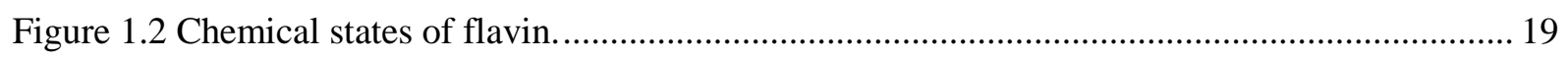

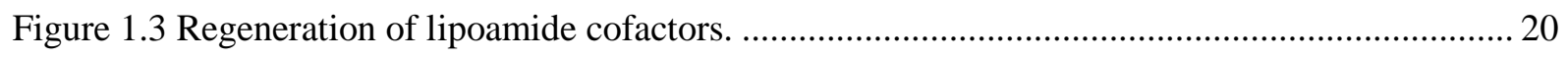

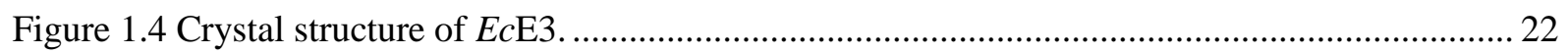

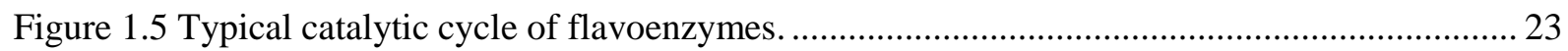

Figure 1.6 Simplified, general reaction scheme of lipoamide dehydrogenases. .................................. 24

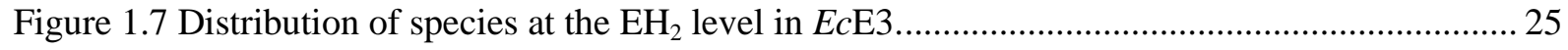

Figure 1.8 Comparison of mixed acid pathway for glucose fermentation. ......................................... 27

Figure 1.9 LOV domain photocycle and light-driven structural rearrangement. ................................. 29

Figure 2.1 Simplified reaction scheme of the EcLip3 reduction by TCEP. ....................................... 43

Figure 2.2 Reaction scheme of the concentration determination of free thiol groups........................... 43

Figure 2.3 MALDI-TOF mass spectrum of the Peptide Calibration Standard ...................................... 53

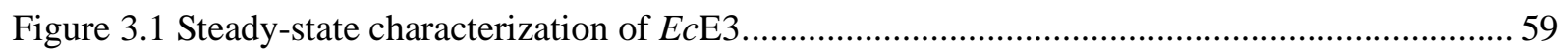

Figure 3.2 Stopped-flow absorbance traces of $E c E 3$ during reduction with NADH........................... 62

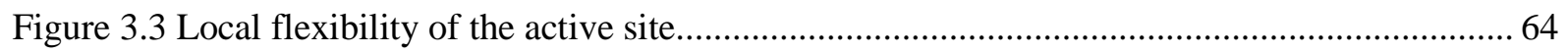

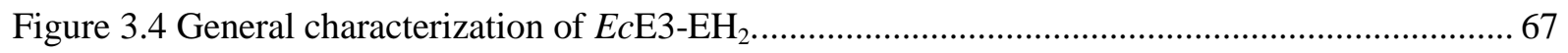

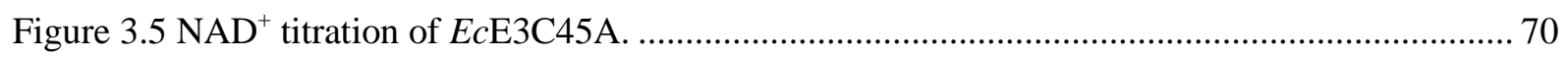

Figure 3.6 The flexibility of cysteine 50 is determined by its hydrogen bond donor............................ 73

Figure 3.7 Evidence for cohesive electron density between Cys50 and FAD-C4a............................... 74

Figure 3.8 Structural model of the covalent C4a-cysteinyl adduct. ..................................................... 75

Figure 3.9 Comparison of the geometries of C4a-cysteinyl adducts in LOV domains and in EcE3 ..... 77

Figure 3.10 In vitro and native substrates of lipoamide dehydrogenases............................................. 78

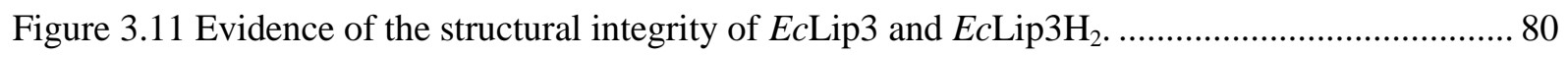

Figure 3.12 Reduction of EcE3 by $E c \mathrm{Lip}_{3} \mathrm{H}_{2}$ under single turnover conditions................................. 82

Figure 3.13 Sequential stopped-flow absorbance analysis of the $E c \mathrm{E} 3$ reduction by $E c \mathrm{Lip}_{3} \mathrm{H}_{2}$ and

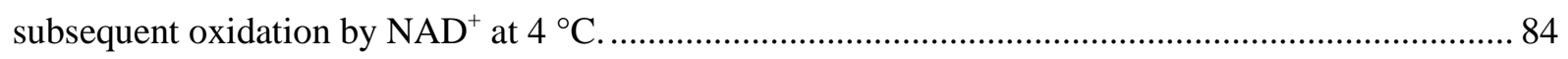

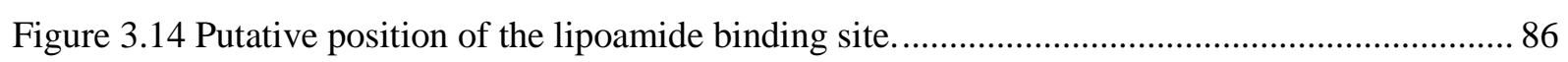

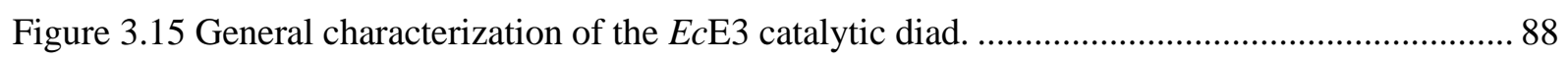

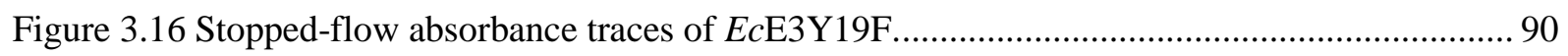

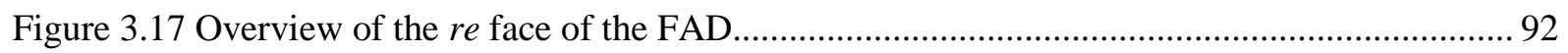

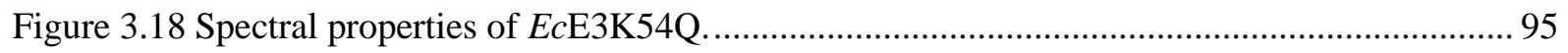

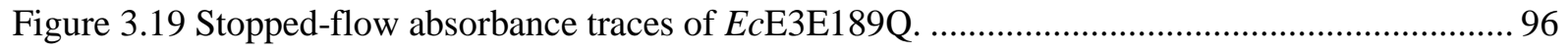

Figure 3.20 Converting $E c E 3$ into a pseudoLOV protein. ............................................................... 100 
Figure 3.21 Comparison of abosorbance changes in variants of pseudoLOVs and LOV domains upon

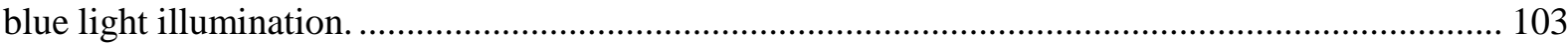

Figure 3.22 UPLC-ESI-TOF-MS analysis of cofactors of bleached and unbleached psLOV............ 105

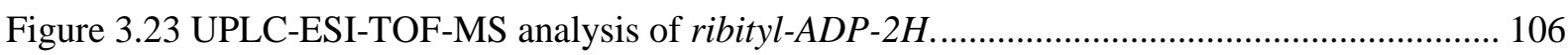

Figure 3.24 The double bond in $F A D-2 H$ and in ribityl-ADP-2H is located the ribityl part. ............ 107

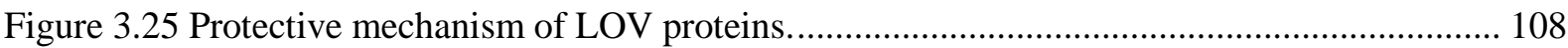

Figure 3.26 Putative FAD derivatives formed upon pseudoLOV photobleaching. ............................ 111

Figure 3.27 Comparison of the H-bonding network around the flavin cofactors in YF1 and $E c$ E3 ... 113

Figure 3.28 Kinetic and spectroscopic comparison between EcE3E354K and EcE3wt.................. 115

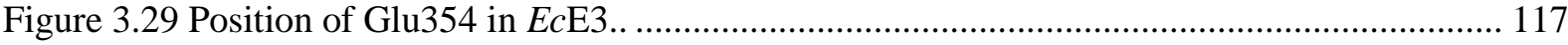

Figure 5.1 Reduction of $E c \mathrm{E} 3$ with NADH under single turnover conditions.................................. 129

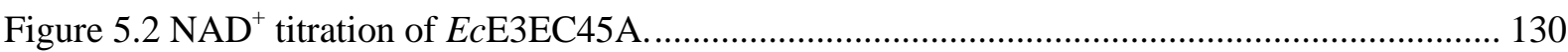

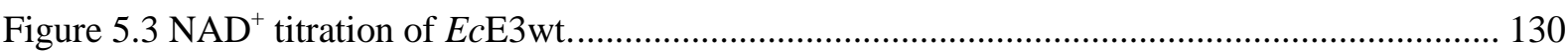

Figure 5.4 Primary amino acid sequences of E3 from different organisms. ....................................... 131

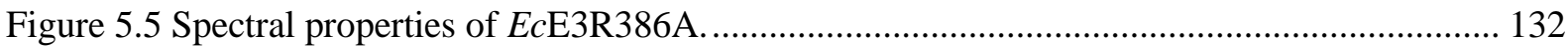

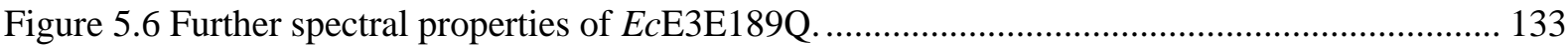

Figure 5.7 Photobleaching of wild type, monothiol or non-thiol variants of $E c E 3$. ......................... 135

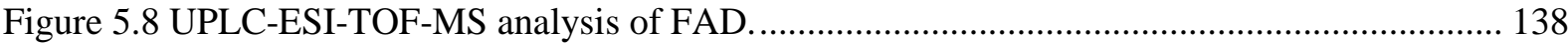

Figure 5.9 UPLC-ESI-TOF-MS analysis of FAD released of $E c \mathrm{E} 3 \mathrm{C} 45 \mathrm{~A} / \mathrm{K} 54 \mathrm{Q}$ by heat precipitation.

Figure 5.10 UPLC-ESI-TOF-MS analysis of FAD released of $E c \mathrm{E} 3 \mathrm{C} 45 \mathrm{~A} / \mathrm{K} 54 \mathrm{Q}$ by TCA precipitation.

Figure 5.11 UPLC-ESI-TOF-MS analysis of FAD released of $E c \mathrm{E} 3 \mathrm{C} 45 \mathrm{~A} / \mathrm{K} 54 \mathrm{Q}$ by acetone precipitation.

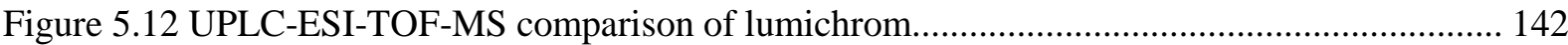

Figure 5.13 Evidence for lumichrom formation in EcE3C45A/K54Q by UPLC-ESI-TOF-MS....... 143

Figure 5.14 Evidence for FAD-2H formation in $E c$ E3C45A/K54Q by UPLC-ESI-TOF-MS............ 144

Figure 5.15 Ribityl-ADP-2H formation in EcE3C45A/K54Q by UPLC-ESI-TOF-MS..................... 145

Figure 5.16 UHPLC-ESI-QTOF-MS/MS analysis of FAD and FAD-2H...................................... 146

Figure 5.17 Determination of the redox potential of EcE3C45A/K54Q …..................................... 147

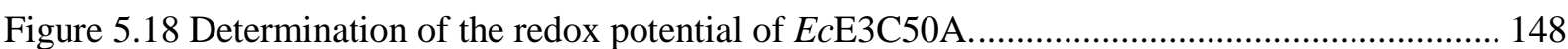

Figure 5.19 Determination of the redox potential of $E c \mathrm{E} 3 \mathrm{C} 45 \mathrm{~S} / \mathrm{C} 50 \mathrm{~S}$............................................. 149

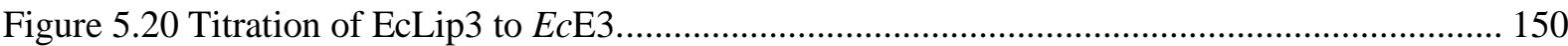

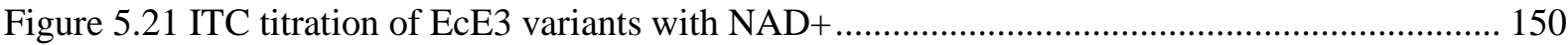

Figure 5.22 The placeholder ability of sulfate ions serving as precipitant........................................ 153

Figure 5.23 Further bending of the flavin cofactor at the two-electron reduced state. ....................... 153

Figure 5.24 B-factor representation of the $E c \mathrm{E} 3$ active site at $2.69 \AA$ A.............................................. 154 


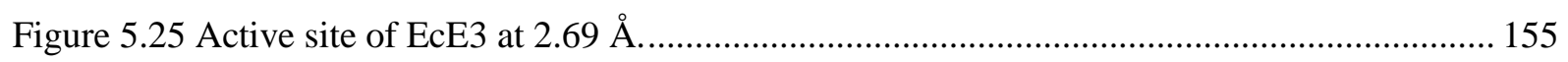




\section{List of Tables}

Table 2.1 List of used primer pairs (Synthesized by Sigma-Aldrich, Taufkirchen). 37

Table 2.2 Overview of buffers used for purification of $E c E 3$ from pGS523

Table 2.3 Overview of buffers used for purification of $E c E 3$ from $p E T 28$ a.

Table 2.4 Overview of buffers used for purification of Lip3 42

Table 2.5 Molar extinction coefficients of $E c \mathrm{E} 3$ and respective variants 44

Table 2.6 Redox pairs for the redox potential titrations. 45

Table 2.7 Spectroscopic characteristics and structures of relevant cofactors. 46

Table 2.8 Acquisition parameters for CD spectroscopy experiments 50

Table 2.9 Possible tryptic fragments of EcLip3 for the lipoylation state analysis 51

Table 2.10 ZipTip preparation for MALDI-TOF samples 52

Table 2.11 Expected and acquired masses of the Peptide Calibration Standard 53

Table 3.1 Steady-state parameters of $E c E 3$ variants involved in the reductive half-reaction. 87

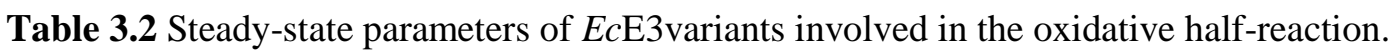
93

Table 5.1 Expected fragmentation pattern of $E c L$ Lip3 after tryptic digestion 137

Table 5.2 Redox potentials of relevant enzymes 147

Table 5.3 Crystallographic data and refinement statistics. 151 


\section{Acknowledgements}

Many people have been helping me during this thesis and I wish to express my deepest thanks to the following:

I would like to thank Prof. Dr. Kai Tittmann for the supervision of this thesis and for contribution of numerous ideas, discussions and inspirations. Especially during difficult stages of the project he showed great support.

I would like to thank Prof. Dr. Ralf Ficner for external co-supervision of this thesis and for being a member of the thesis committee. I would also like to thank him for the generous ability to use the instruments and facilities of his department.

I would like to thank Prof. Dr. Marina Rodnina for taking part in the thesis committees and for good advices concerning kinetic issues.

Furthermore I am grateful to Dr. Fabian Commichau, Jun. Prof. Ricardo Mata and Dr. Manfred Konrad for being members of the extended thesis committee.

I am deeply thankful to Dr. Piotr Neumann for his continuous support in all theoretical and practical aspects of protein crystallography. Especially, I would like to thank him for carrying out the majority of the x-ray data processing work. I would also like to thank him for suggestions and comments in the field of structural biology in general - and life in general.

Dr. Florian Brodhun and Dr. Piotr Neumann I acknowledge for proofreading of the thesis.

Prof. Dr. Andreas Möglich and Dr. Ralph Diensthuber I would like to thank for a perfect and exciting cooperation in the field of flavoproteins and their photochemistry. In addition, I wish you all the best at your new positions.

I acknowledge Prof. Dr. Ivo Feussner for the generous ability to use the instruments of his department. Moreover, I would like to thank Dr. Kirstin Feussner for the mass spectrometric analysis of several flavin cofactors and many good advices concerning MS sample preparation and MS analysis.

Prof. Dr. Robert Bittl and Christopher Engelhard I acknowledge for several EPR control measurements during the "pseudoLOV"-project.

Prof. Dr. Bruce Palfey and Lena-Sophie Dreher I acknowledge for the determination of redox potentials of selected $E c \mathrm{E} 3$ variants. 
Dr. Achim Dickmanns and Michael Franke I would like to thank for the easy access to the pipetting robot and concomitant crystallization trials.

Dr. Kathrin Schröder-Tittmann I would like to thank for a nice collaboration in the structural and functional characterization of the PDH complexes from human and E. coli.

I acknowledge Dr. Oliver Valerius for his brilliant assistance in the field of MALDI-TOF mass spectrometry. He made it possible to measure and to evaluate data whenever I needed and thus contributed to the flow of the work.

Prof. Dr. Reinhard Jahn and Dr. Angel Perez Lara I would like to thank for very nice cooperation in the field of Isothermal Titration Calorimetry.

Prof. Dr. Holger Stark, Dr. Ashwin Chari and David Haselbach I acknowledge for our close collaboration embedded in the SFB860 and the ProteoPlex project.

Gerd Mader, Christoph Mader, Daniel Weinrich and Malte Bürsing I would like to thank for their technical support.

I would particularly like to thank Dr. Danilo Meyer for his supervision during the Master Thesis. He always took his time to discuss certain issues about the project which made the work much more comfortable.

I would like to thank all former and present members of the bioanalytics / molecular enzymology department for their help in the lab and the great working atmosphere.

Finally, I am deeply grateful to my parents for their endless support during my studies and life in general. 


\section{Abbreviations}

$\AA$

$\mathrm{A}_{260}$ or $\mathrm{A}_{280}$

$\mathrm{ACN}$

Ala

app

Apo

Arg

Asn

Asp

ATP

asu

bp

Carb

CD

$\mathrm{cm}$

cps

CV

Cys

Da

DHB

$\varepsilon$

Ec

$E c \mathrm{E} 1$

$E c \mathrm{E} 2$

$E c \mathrm{E} 3$

EcLip3

E. coli

EDTA

$\mathrm{E}_{\mathrm{ox}}$

$\mathrm{EH}_{2}$

$\mathrm{EH}_{4}$

EIC

EPR

ESI
Ångström

absorption at 260 or $280 \mathrm{~nm}$

acetonitrile

alanine

apparent

apoenzyme, enzyme without cofactor

arginine

asparagine

asparaginic acid

adenosine triphosphate

asymmetric unit

base pairs

carbenicillin

circular dichroism

centimeter

counts per second

column volume

cysteine

dalton

2,5-dihydroxybenzoic acid

molar extinction coefficient

Escherichia coli

Escherichia coli pyruvate dehydrogenase

Escherichia coli dihydrolipoyl transacetylase

Escherichia coli dihydrolipoyl dehydrogenase

innermost lipoyl domain of Escherichia coli dihydrolipoyl transacetylase

Escherichia coli

ethylendiamintetraacetate

oxidized form $E$. coli lipoamide dehydrogenase

two-electron reduced form $E$. coli lipoamide dehydrogenase

four-electron reduced form E. coli lipoamide dehydrogenase

extracted ion chromatogram

electron paramagnetic resonance

electrospray ionization 
FA formic acid

FAD flavin adenine dinucleotide

FMN flavin mononucleotide

Gln glutamine

Glu glutamic acid

Gly glycine

HEPES 4-(2-hydroxyethyl)-1-piperazineethanesulfonic acid

His histidine

Ile isoleucine

IPTG isopropyl- $\beta$-D-thiogalactoside

ITC isothermal titration calorimetry

$k \quad$ rate constant

$K_{\mathrm{D}} \quad$ dissociation constant

$K \quad$ equilibrium constant

KPP potassium phosphate buffer

$\lambda \quad$ wavelength

LB media Luria-Bertani-Media

Leu leucine

Lys lysine

$\mathrm{NADH} / \mathrm{NAD}^{+}$nicotinamide adenine dinucleotide (reduced form/oxidized form)

MS mass spectrometry

NMR nuclear magnetic resonance

obs observed

OD optical density

PDHc pyruvate dehydrogenase complex

PDB protein data bank

$\mathrm{pH} \quad$ negative logarithm of $\mathrm{H}^{+}$concentration

Phe phenylalanine

PEG polyethylene glycol

Pro proline

PMSF phenylmethylsulfonylfluorid

rpm revolutions per minute

Ser serine

SDS-PAGE sodium dodecylsulfate polyacrylamide gelelektrophoresis 
TCA trichloroacetic acid

TCEP Tris-(2-carboxyethyl)phosphine hydrochloride

ThDP thiamin diphosphate

Thr threonine

TOF time of flight

Tris tris (hydroxymethyl)-aminomethan

Trp tryptophane

TWC total wavelength chromatogram

Tyr tyrosine

U unit

UV/Vis ultraviolet and visible fraction of electromagnetic radiation

Val valine 


\section{Introduction}

\subsection{Flavin and its biologically Active Derivatives}

Enzymes are versatile biocatalysts present in every organism in all domains of life. Understanding their mode of actions at an atomic level is of primary interest since enzymes are both potential drug targets for the pharmaceutical industry and potential catalysts under physiological conditions for the chemical industry. A subclass are flavoenzymes, enzymes that bind derivatives of riboflavin (vitamin B2), and of which a plethora has already been identified and characterized (Palfey and Fagan 2010). Flavoenzymes also harbor the aforementioned versatility with respect to the ability of catalyzing chemical reactions. Hence, they are involved in a multitude of biological processes including energy production, light-induced signal transduction and DNA repair to name only a few (Conrad, Manahan, and Crane 2014; Palfey and Fagan 2010). The probably most fascinating feature of flavoenzymes is their aptitude to act as carriers for reducing equivalents thereby transferring electrons from a donor reactant to an acceptor reactant. Thus, flavoenzymes play a pivotal role in metabolism acting as key players in the respiratory chain.

Flavins consist of a tricyclic isoalloxazine moiety attached to a ribityl chain at the N10 atom (Figure 1.1). The most prominent enzyme bound flavin derivatives are flavin mononucleotide (FMN) and flavin adenine dinucleotide (FAD). FMN results from phosphorylation at the 5'-OH atom of the ribityl chain and FAD is the product from an esterification of AMP and FMN. Generally, flavins and enzymes form noncovalent complexes characterized by small binding constants making a covalent attachment of the cofactor to the enzyme almost unnecessary. The tight binding is achieved by interactions of the protein with the ribityl chain and the 5'-phosphate group, and, in case of FAD, by interactions of a secondary structure element (Rossmann fold) with its ADP portion (Hanukoglu 2015). However, covalent attachment occasionally occurs to form the holoprotein via the 8-a (methyl) or the C6 atom of the dimethylbenzene moiety (Palfey and Fagan 2010) or at both sides at once (Shoyama et al. 2012). 


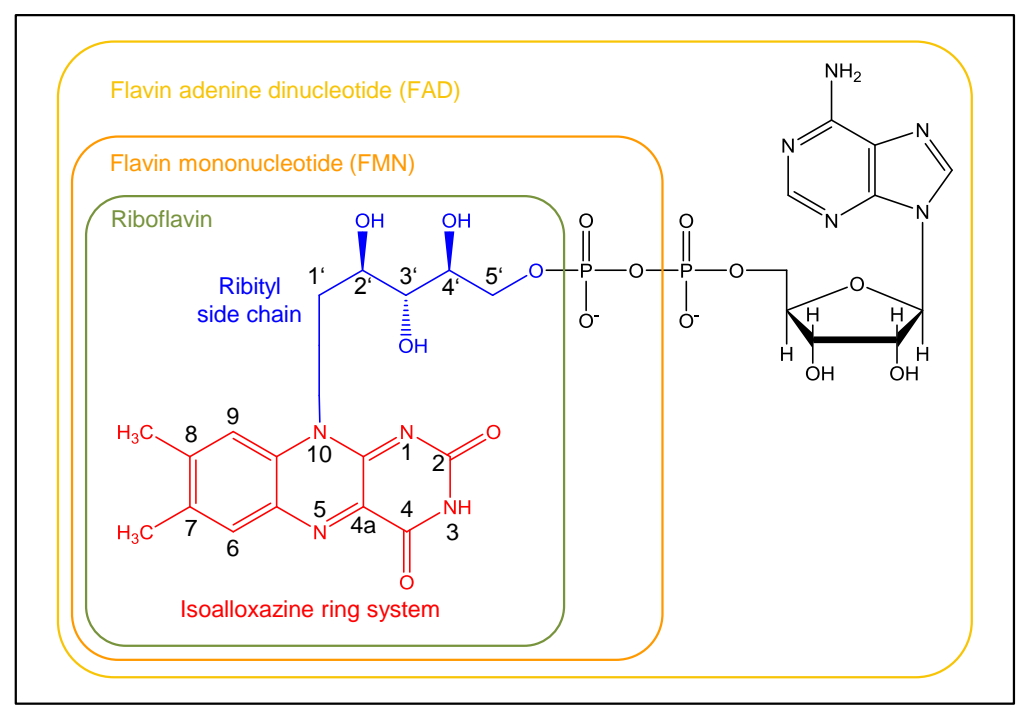

Figure 1.1 Flavin structure and nomenclature.

\subsection{Reactivity of Flavins}

The reactive part of flavins is the heterocyclic isoalloxazine ring system and three stable oxidation states can be assigned to the cofactor: fully oxidized, semiquinone (1-electron reduced), and hydroquinone (2-electron reduced) (Figure 1.2). The ring system is planar in solution and also in most protein structures, but it was also demonstrated that the flavin redox potential can be tuned by conformational effects leading to deviations from this planarity. Especially bending of the ring system along the N5-N10 axis was demonstrated to influence the ability to accept one or two electrons (Hasford, Kemnitzer, and Rizzo 1997; Lyubimov et al. 2007; Walsh and Miller 2003) making the electron-deficient isoalloxazine a target for nucleophilic attack at N5, C4a, and C6 (Palfey and Fagan 2010). Oxidized flavins harbor a shiny yellow color and are highly polarizable whereas the resulting reactivity is mainly influenced by interactions with the respective enzyme.

The single-electron reduced state is called semiquinone which is thermodynamically unstable in aqueous solutions (Gibson, Massey, and Atherton 1962). However, some proteins provide surroundings drastically increasing the lifetime of the semiquinone whereas others completely prevent its formation (Beel et al. 2012; Massey and Palmer 1966). Flavin semiquinones either exist as neutral (blue) radicals or as anionic (red) radicals in which the free electron is delocalized over the isoalloxazine (Ehrenberg, Müller, and Hemmerich 1967; Müller et al. 1970). EPR spectroscopy demonstrated that the highest spin density can be found at $\mathrm{C} 4$ a for the neutral radical and at $\mathrm{N} 5$ for the anionic radical. During redox reactions they can act as single-electron donors/acceptors or also form radical pairs (Palfey and Fagan 2010).

The flavin hydroquinone is pale yellow. It is the two-electron reduced state which is mostly planar and thus antiaromatic. However, the aforementioned bending along the N5-N10 axis was also observed in 
small-molecule structures and some protein structures, but to a much more pronounced extent $\left(>30^{\circ}\right)$ (Palfey and Fagan 2010). Bending is most likely determined by protonation of N1 since the protonated hydroquinone was shown to adopt a butterfly conformation whereas the anionic hydroquinone is planar (Rizzo 2001; Rodríguez-Otero et al. 2002; Zheng and Ornstein 1996). In contrast to semiquinones, hydroquinones can react as single-electron donors, as hydride donors, or as nucleophiles at N5 or C4a (Palfey and Fagan 2010).

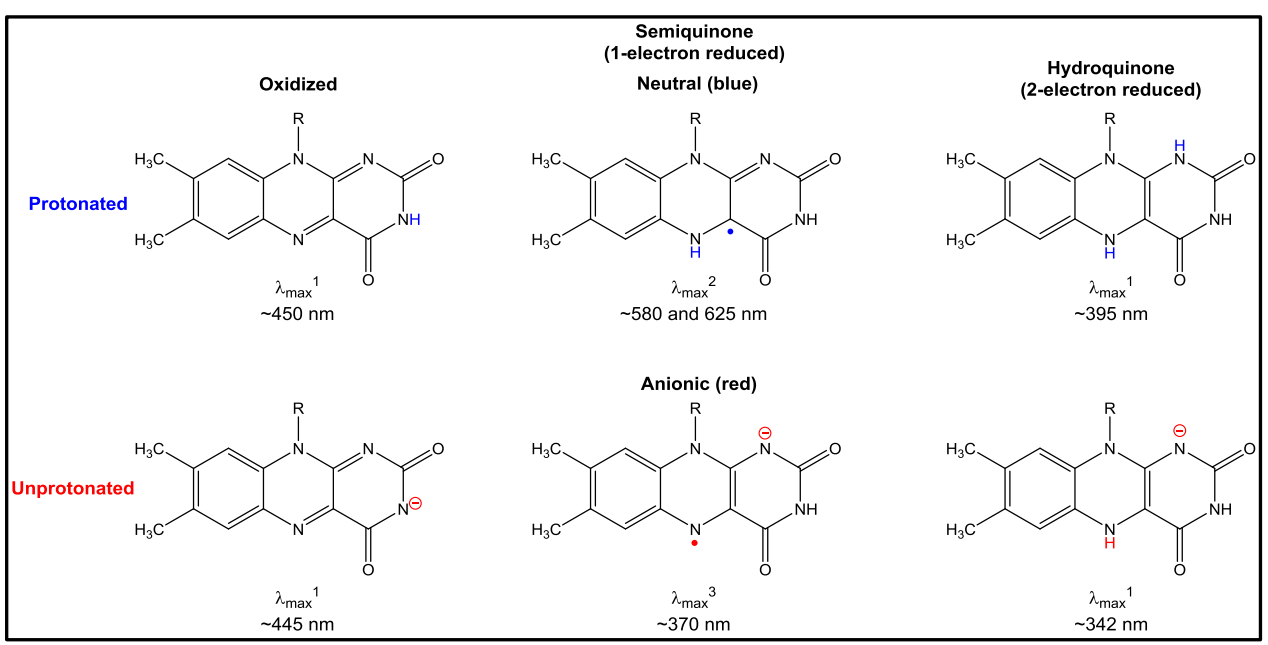

Figure 1.2 Chemical states of flavin.

The absorption maxima are approximate values and may slightly differ due to the respective enzyme surrounding. Wavelengths below $300 \mathrm{~nm}$ were not taken into account. ${ }^{1}$ (Ghisla et al. 1974) ${ }^{2}$ (Hitomi et al. 1997; Massey and Palmer 1966) ${ }^{3}$ (Choong and Massey 1980; Talfournier et al. 2001b).

\subsection{Biological Functions of Lipoamide Dehydrogenases}

Lipoamide dehydrogenases (EC 1.8.1.4) belong to the flavoenzymes family of pyridine nucleotidedisulfide oxidoreductases which share several structural and mechanistic properties (Palfey and Fagan 2010). This family further includes: glutathione reductase (EC 1.6.4.2), mycothione reductase (EC 1.8.1.15), trypanothione reductase (EC 1.8.1.12), and mercuric reductase (EC 1.16.1.1), all pivotal for maintaining metabolic and cellular functions. Lipoamide dehydrogenases are part of ketoacid dehydrogenase multienzyme complexes and the glycine cleavage system and catalyze the reoxidation of lipoamide cofactors with concomitant transfer of reducing equivalents to $\mathrm{NAD}^{+}$thereby generating NADH (Figure 1.3) (Perham 2000; Reed 1974, 2001). 


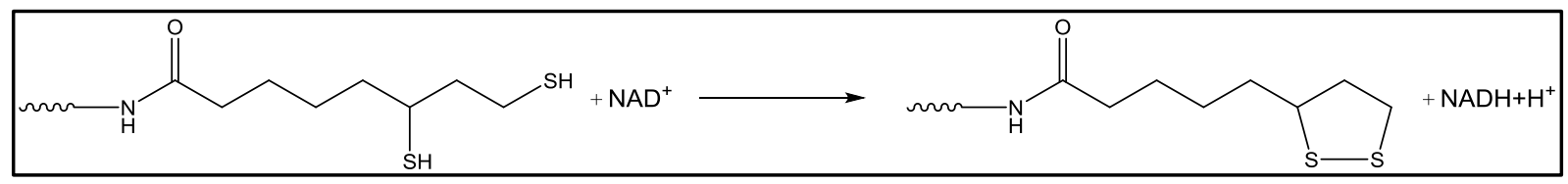

Figure 1.3 Regeneration of lipoamide cofactors.

Lipoamide dehydrogenases catalyze the $\mathrm{NAD}^{+}$-dependent oxidation of the dihydrolipoyl cofactors that are covalently linked to the acyltransferase components of multienzyme complexes.

As part of the pyruvate dehydrogenase multienzyme complex (PDHc) lipoamide dehydrogenases are directly involved in energy metabolism by linking glycolysis and citric acid cycle. PDHc is present in a multitude of organisms, namely in respiratory eubacteria and the mitochondria of eukaryotes (Bates et al. 1977; Patel and Roche 1990; Perham 2000). It catalyzes the irreversible conversion of pyruvate, co-enzyme A and $\mathrm{NAD}^{+}$into $\mathrm{CO}_{2}$, acetyl-CoA and $\mathrm{NADH}$. Acetyl-CoA then serves as a precursor for the citric acid cycle and the biosynthesis of fatty acids and steroids, while NADH eventually feeds reducing equivalents into the respiratory chain for oxidative phosphorylation (ATP synthesis) (Patel and Roche 1990). PDHc consists of multiple copies of three major enzyme components: a thiamine diphosphate-dependent pyruvate dehydrogenase (E1 (EC 1.2.4.1)), a dihydrolipoamide transacetylase (E2 (EC 2.3.1.12)), which carries lipoyl groups covalently linked to the $\varepsilon$-amino group of lysine residues (N6-(lipoyl)-lysine, lipoamide cofactor), and the aforementioned lipoamide dehydrogenase (E3) (Korotchkina and Patel 2001; Patel and Korotchkina 2001). An important and outstanding ability of PDHc is the mechanism of covalent substrate channeling between the different components. The N6-(lipoyl)-lysine conjugate is structurally highly flexible. A $14 \AA$ "swinging arm" permits active site coupling between the E1, E2 and E3 components by movement of the lipoyl moiety itself and by additional movement of the whole protein domain. This domain (Lip-domain) carries the lipoamide cofactor thus providing an appendage that is capable to bridge the physical gaps between the active centers on the different components (Perham and Reche 1998; Perham 2000).

In all PDHc, the reaction cycle is catalyzed by those enzymes and can be subdivided into several steps. Initially, E1 binds pyruvate and catalyzes its irreversible decarboxylation yielding 2-hydroxyethylthiamine diphosphate. Afterwards, E1 transfers the C2-fragment to the oxidized dithiolane ring of the lipoamide cofactor of E2. This reductive acetylation results in the formation of a high-energy thioester linkage between a sulfur atom of the reduced lipoamide dithiolane moiety and the acetyl fragment derived from pyruvate (Patel and Roche 1990). In the following, E2 itself catalyzes the acyl group transfer from reduced S-acetyldihydrolipoamide to Coenzyme A leading to the formation of acetylCoA (Akiyama and Hammes 1981). The reduced lipoamide cofactor must be oxidized in order to permit subsequent cycles of catalysis. This reaction step is catalyzed by lipoamide dehydrogenases under transient reduction of an intrinsic disulfide bond and further transfer of reducing equivalents to 
the enzyme-bound flavin (Argyrou, Blanchard, and Palfey 2002). Generation of NADH by E3 finally completes the reaction cycle.

\subsection{Three Dimensional Structure of E. coli Lipoamide Dehydrogenase}

Several crystal structures of lipoamide dehydrogenases from various species have been solved over the years (Brautigam et al. 2005; Mattevi et al. 1993; Mattevi, Schierbeek, and Hol 1991); that of the E. coli enzyme recently (Chandrasekhar et al. 2013). The tertiary structures reflect very high similarity and only minor structural differences can be observed in surface exposed loop regions (Chandrasekhar et al. 2013). In addition, lipoamide dehydrogenase is structurally homologous to glutathione reductase, a further member of the flavoenzyme disulfide reductases. All described lipoamide dehydrogenases are homo-dimeric enzymes with two active sites formed at the dimer interface (Figure 1.4A). Each monomer thereby consists of four domains. A large N-terminal FAD-binding domain (residues 1-149; referring to E. coli numbering) which contacts the three remaining domains: the NAD-binding domain (150-282), the central domain (283-350), and the interface domain (351-474) (Figure 1.4B). The dimeric form of E. coli lipoamide dehydrogenase is based on its active site architectures involving residues of both monomers, respectively. The redox active disulfide bridge Cys45 to Cys50 which directly interacts with lipoamide cofactors is located at the dimer interface on the si face of the tightly but non-covalently bound flavin (Figure 1.4C). Above this electron transfer site, at the re face of the flavin, the $\mathrm{NAD}^{+} / \mathrm{NADH}$ binding site is housed. The highly conserved catalytic diad (His445 and Glu450) from the adjacent monomer involved in acid/base chemistry completes the active site (Benen, Van Berkel, Dieteren, et al. 1992; Kim and Patel 1992b). 


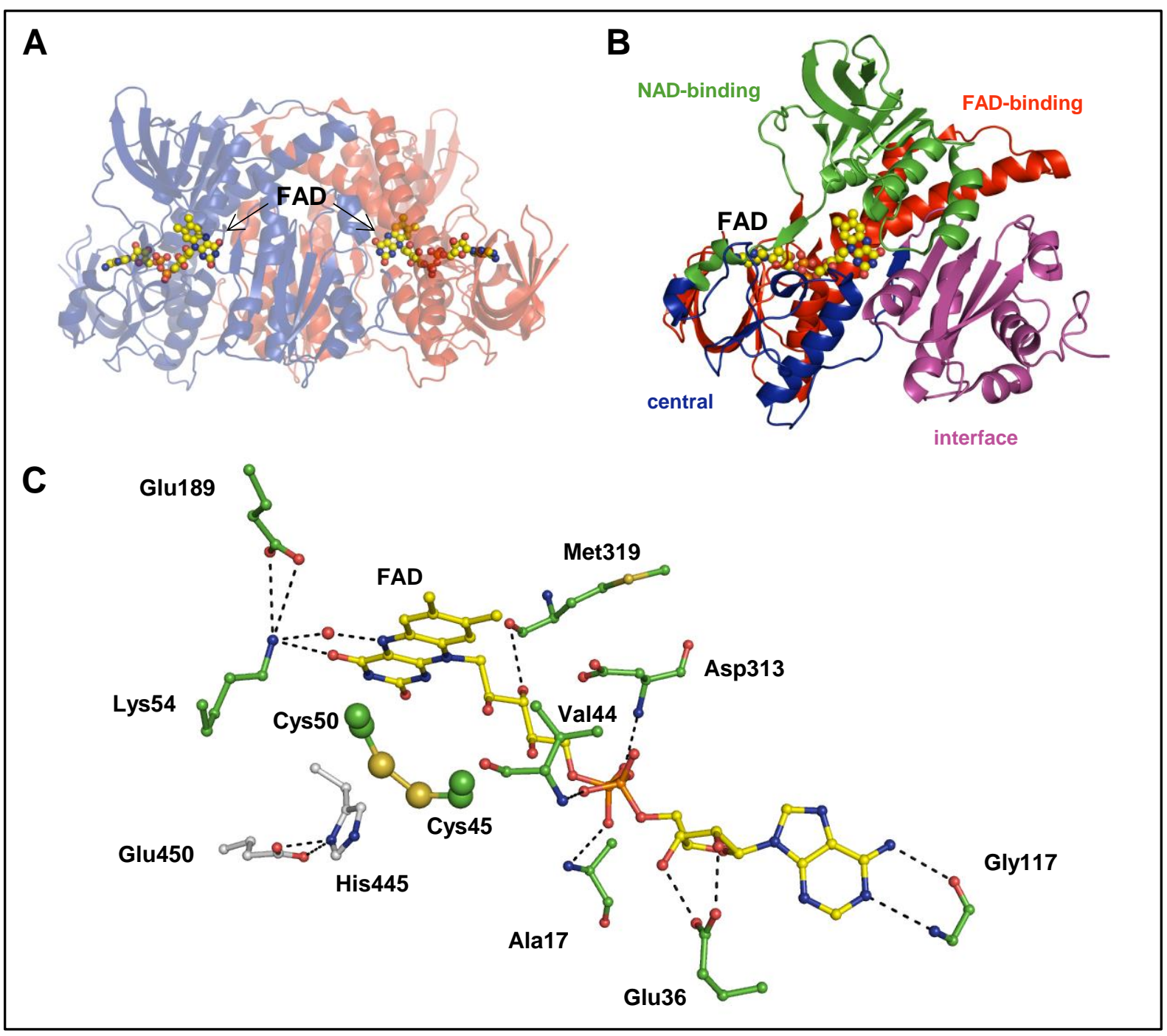

Figure 1.4 Crystal structure of $E c \mathrm{E} 3$.

(A) Structure of the biologically functional dimer of $E c E 3$ (Chandrasekhar et al. 2013) in cartoon representation with the bound flavin cofactor (ball representation). Individual monomers are shown in blue and red. (B) Domain structure of $E c E 3$ in cartoon representation: N-terminal FAD-binding (red), NAD-binding domain (green), central domain (blue) and C-terminal interface domain (purple). (C) Interactions of the FAD molecule with E3. The enzymatic disulfide (ball representation) is located on the $s i$ face of the flavin. The nearby histidine is involved in acid/base chemistry. Interactions are indicated by dashed lines. Residues from the neighboring subunit are shown in light grey. 


\subsection{Catalytic Mechanism of E. coli Lipoamide Dehydrogenase}

Generally, the catalytic cycles of flavoenzymes can be divided into reductive and oxidative halfreactions (Figure 1.5). In the reductive half-reaction, the oxidized flavoenzyme is reduced by the first substrate, yielding in a reduced flavoenzyme. In the oxidative half-reaction, a second substrate oxidizes the reduced enzyme (Palfey and Fagan 2010). This usually results in ping-pong kinetic mechanisms since often the product of the reductive half-reaction dissociates prior to the beginning of the oxidative half-reaction (Palfey and Fagan 2010; Reed 1973).

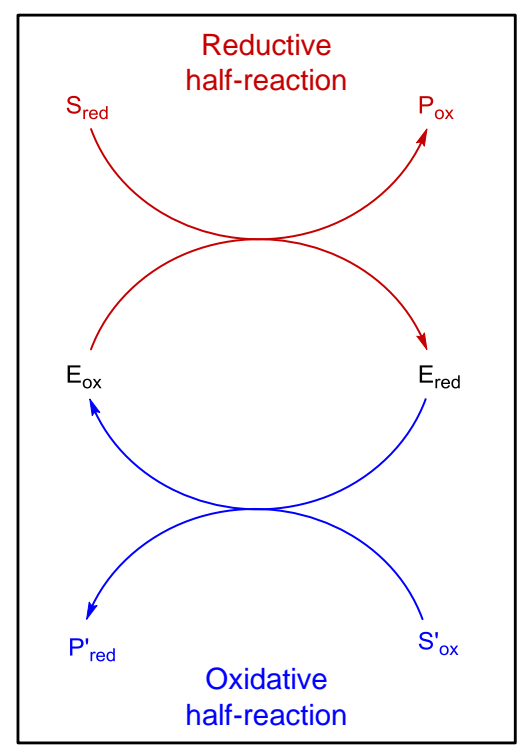

Figure 1.5 Typical catalytic cycle of flavoenzymes.

The ping-pong kinetic mechanism of $E c \mathrm{E} 3$ involves two half-reactions, each completely reversible and has been studied in detail (Argyrou et al. 2003, 2002; Sahlman and Williams 1989; Wilkinson and Williams 1979, 1981). In the reductive half-reaction, the substrate dihydrolipoamide binds to the oxidized state of EcE3 $\left(\mathrm{E}_{\mathrm{ox}}\right)$, and Cys45 forms a mixed disulfide with the substrate. Transfer of electrons yield to thiolate formation of Cys50 and thus the two-electron reduced state $\left(\mathrm{EH}_{2}\right)$. This reaction is general-acid-base catalyzed by an essential and conserved catalytic diad located at the Cterminus of the adjacent monomer of the homo-dimeric enzyme (Benen, Van Berkel, Dieteren, et al. 1992; Kim and Patel 1992b). Release of lipoamide completes the reductive half-reaction (Figure 1.6). In the oxidative half-reaction, $\mathrm{NAD}^{+}$binds to the two-electron reduced state of the enzyme $\left(\mathrm{EH}_{2}\right)$. This induces an internal electron transfer from the redox active disulfide towards the flavin cofactor yielding in a transiently formed $\left[\mathrm{NAD}^{+}-\mathrm{FADH}_{2}\right]$ intermediate. The internal electron transfer is thought to proceed via a covalent adduct between Cys50 and the $\mathrm{C} 4 \mathrm{a}$ position of the $\mathrm{FAD}$, which is termed 
"FAD-C4a-cysteinyl adduct" (Miller et al. 1990; Thorpe and Williams 1976a, 1976b). The flavin is immediately reoxidized by $\mathrm{NAD}^{+}$generating $\mathrm{NADH}$ and the oxidized form of the enzyme $\left(\mathrm{E}_{\mathrm{ox}}\right)$. Release of NADH completes the oxidative half-reaction preparing the enzyme for another round of catalysis.

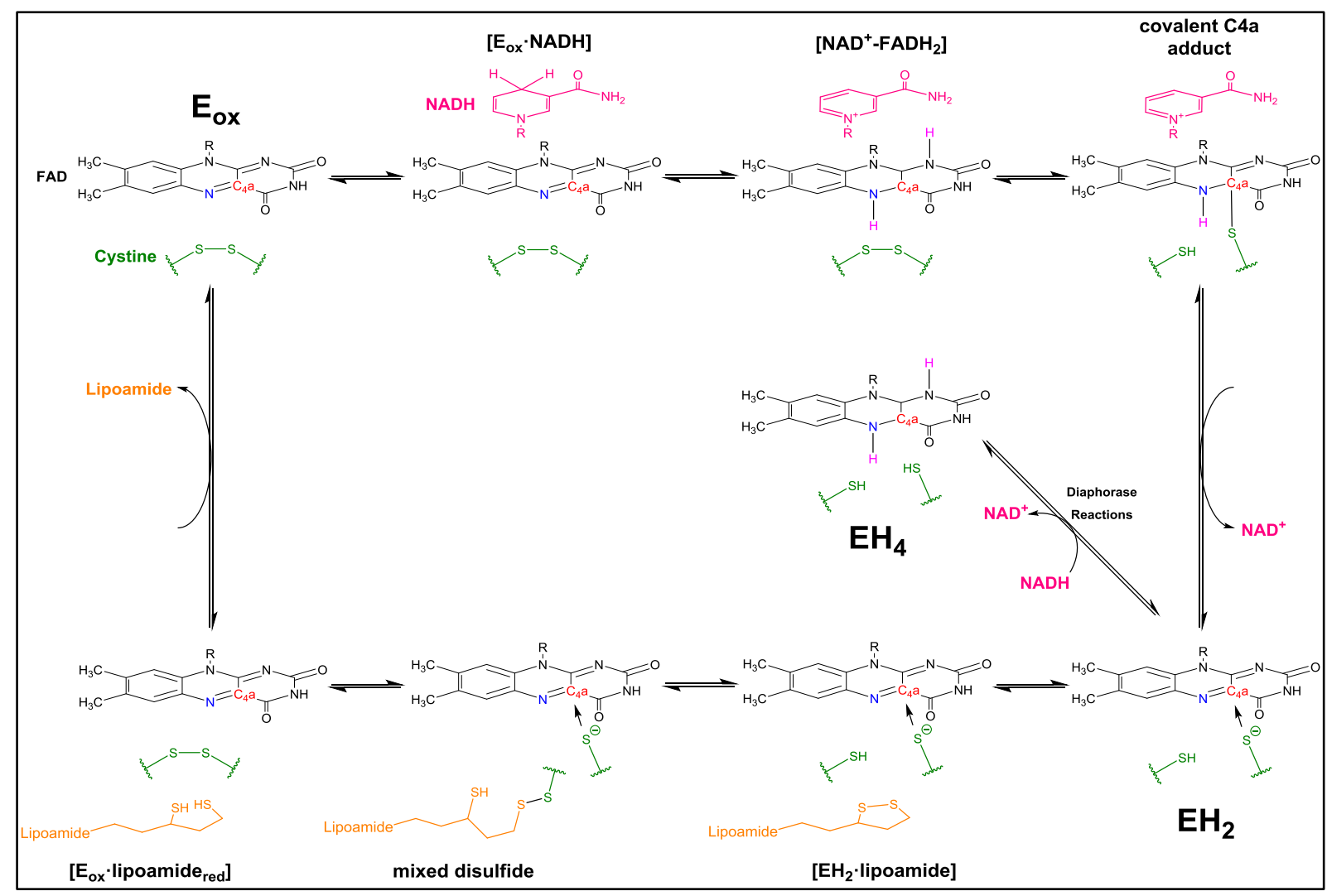

Figure 1.6 Simplified, general reaction scheme of lipoamide dehydrogenases.

E3-catalyzed oxidation of reduced lipoamide cofactors and subsequent generation of NADH: $\mathrm{R}=$ ribityl-ADP, $\mathrm{E}_{\mathrm{ox}}$ oxidized, $\mathrm{EH}_{2}=$ two-electron reduced and $\mathrm{EH}_{4}=$ four-electron reduced state of the enzyme. Reaction intermediates are indicated. For explanation see text.

During catalysis, the enzyme cycles between the oxidized and two-electron reduced states. However, the E. coli enzyme is easily overreduced by a small molar excess of NADH to the four-electron reduced state $\left(\mathrm{EH}_{4}\right)$ (Williams 1965). In this catalytically inactive dead-end complex, the redox-active disulfide and the flavin are reduced (Figure 1.6). $\mathrm{NAD}^{+}$has an activating effect because the pyridine nucleotide can oxidize the dead-end complex, increasing the amount of catalytic active $\mathrm{EH}_{2}$ (Matthews, Ballou, and Williams 1979). The severe susceptibility to overreduction by NADH, and thus NADH inhibition, is based on the redox potentials of the $\mathrm{EH}_{2}$ and $\mathrm{EH}_{4}$ states. At first, comparison of the reduction potentials for the two redox couples of lipoamide dehydrogenases from E. coli (264 and $-317 \mathrm{mV}$, respectively) (Wilkinson and Williams 1979), pig heart (-280 and $-346 \mathrm{mV}$ ) (Matthews and Williams 1976), and M. tuberculosis (-309 and $-382 \mathrm{mV}$ ) (Argyrou et al. 2002) 
demonstrate that the E. coli enzyme has the most positive $\mathrm{E}_{\mathrm{ox}} / \mathrm{EH}_{2}$ and $\mathrm{EH}_{2} / \mathrm{EH}_{4}$ redox couples. Secondly, the redox potentials of the $\mathrm{EH}_{2}$ and $\mathrm{EH}_{4}$ pairs are closer than in the enzymes from the aforementioned organisms. Consequently, due to the closer potentials, the $\mathrm{EH}_{2}$ state of the E. coli enzyme exists as a fast equilibrating mixture of three species: the oxidized flavin-dithiol species (Figure 1.7I), the oxidized flavin-thiolate charge transfer (II), and the reduced flavin-disulfide (III), all harboring different spectroscopic properties (Wilkinson and Williams 1979). The physiological consequences of the NADH inhibition of $E c \mathrm{E} 3$ are highlighted in a following chapter (1.6).

Figure 1.7 Distribution of species at the $\mathrm{EH}_{2}$ level in $E c \mathrm{E} 3$. Species II has a characteristic red color derived from the charge transfer interaction between Cys50-thiolate and FAD - adapted from (Wilkinson and Williams 1979).

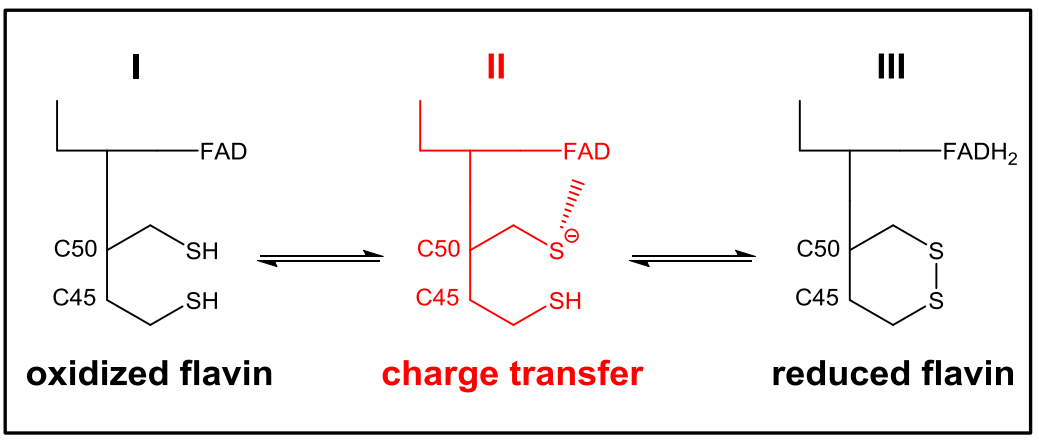

\subsection{The Physiological Importance of E. coli Lipoamide Dehydrogenase}

As a facultative heterotroph Escherichia coli is able to grow under aerobic and anaerobic conditions. While growing aerobically glucose is metabolized using glycolysis, PDH complex and citric acid cycle. The NADH generated during these reactions is oxidized by the final electron acceptor oxygen. In contrast, under anaerobic growth glycolytic intermediates serve as electron acceptors to maintain the redox balance. However, this leads to an increased $\mathrm{NADH} / \mathrm{NAD}^{+}$ratio compared to aerobic growth (Graef et al. 1999; Snoep et al. 1993). In both growth modes, pyruvate is the key intermediate in the catabolism in E. coli. Its subsequent conversion by either pyruvate formate lyase (PFL) or the PDH complex can be claimed as the major switch between mixed acid fermentation and the citric acid cycle and subsequent respiration. Though PDH activity is obligate for aerobic growth of the bacterium, its activity was also detected in cell extracts grown under anaerobic conditions albeit at a very low or nearly undetectable amount (Graef et al. 1999; Smith and Neidhardt 1983; Snoep et al. 1993).

The regulation of E. coli $\mathrm{PDH}$ activity is rather complex, but the E1 and the E3 components have been identified as main targets for regulation several decades ago (Hansen and Henning 1966). Whereas the mammalian E1 is regulated by phosphorylation (Cooper, Randle, and Denton 1974; Hucho et al. 1972; 
Seifert et al. 2007), the availability of metabolic intermediates regulate the E. coli E1 activity. On the one hand acetyl-CoA inhibits E1 (Hansen and Henning 1966) on the other hand glycolytic intermediates enhance the PDH activity presumably by accelerating the carbon flow towards the citric acid cycle (Shen et al. 1968). As the most efficient activator fructose-1,6-bisphosphate was identified, however PDH activity is also enhanced by fructose-6-P, glycerolaldehyde-3-P, phosphoenolpyruvate, dihydroxyacetone-P and glucose-6-P.

$E c \mathrm{E} 3$ is regulated by the ratio of reduction equivalents rather than by glycolytic intermediates. As described by WILKINSON \& WILLIAMS EcE3 suffers from severe product inhibition by NADH due to reduction of the catalytically active two-electron reduced enzyme intermediate $\left(\mathrm{EH}_{2}\right)$ to the inactive four-electron reduced form $\left(\mathrm{EH}_{4}\right)$ while $\mathrm{NAD}^{+}$is able to oxidize this dead-end complex, increasing the amount of $\mathrm{EH}_{2}$ (Wilkinson and Williams 1981). This inhibitory effect of NADH not only holds true for the isolated $E c \mathrm{E} 3$ but also for the entire E. coli $\mathrm{PDH}$ (Bisswanger 1981; Hansen and Henning 1966; Wilkinson and Williams 1981). Thus, PDH activity is also modulated by the NAD ${ }^{+} / \mathrm{NADH}$ pool affecting the redox state of $E c \mathrm{E} 3$.

As aforementioned, anaerobically growing E. coli metabolizes pyruvate with the help of the pyruvateformate lyase complex (FHL) to acetyl-CoA whereas the reducing equivalents are temporary stored as formate (Clark 1989). Succinate is only a minor fermentation product (Fraenkel 1996) and lactate is mainly formed under strong acidic growth conditions (Clark 1989). Formate is finally removed by disproportionation to $\mathrm{H}_{2}$ and $\mathrm{CO}_{2}$ without influencing the $\mathrm{NADH} / \mathrm{NAD}^{+}$ratio (Rosentel et al. 1995). The remaining NADH derived from preceding glycolytic steps is then oxidized using acetyl-CoA as electron acceptor with the penultimate production of ethanol by aldehyde-alcohol dehydrogenase (Clark 1989). However, the reduction of acetyl-CoA to ethanol requires two equivalents of NADH and therefore the degradation of the remaining acetyl-CoA molecule to acetate via acetyl phosphate by phosphotransacetylase and acetate kinase is mandatory. Hence, the fermentation profile of E. coli harbors equimolar amounts of ethanol and acetate (Figure 1.8A).

E. coli strain AH242 lacks pyruvate-formate lyase $(p f l B)$ and fermentative lactate dehydrogenase $(l d h A)$ and is therefore defective for anaerobic growth (Clark 1989; Kim, Ingram, and Shanmugam 2007). Kim et al. isolated and described an E. coli mutant (SE2378) derived of strain AH242 that produced ethanol as the main fermentation product (Kim et al. 2007). The mutation was identified as a single change in the $E c \mathrm{E} 3$ amino acid sequence (Glu354 $\rightarrow$ Lys354). This mutation led to a reduced sensitivity of $E c E 3$ - and thus the PDH complex - towards NADH inhibition leading to PDH activity under anaerobic growth conditions which concomitantly changed the fermentation profile of mutant SE2378 (Figure 1.8B) (Kim, Ingram, and Shanmugam 2008). With the PDH being active under anaerobic conditions mutant SE2378 produces four NADH molecules during the degradation of one molecule glucose to two acetyl-CoA molecules. In order to maintain the redox balance these four $\mathrm{NADH}$ molecules are then reoxidized by reduction of the two acetyl-CoA molecules to two ethanol 
molecules by the alcohol/acetaldehyde dehydrogenase ( $a d h E)$. This study further underpinned the physiological importance of $E c E 3$ and showed that even a single amino acid mutation has the potential to change a bacterium's growth behavior.

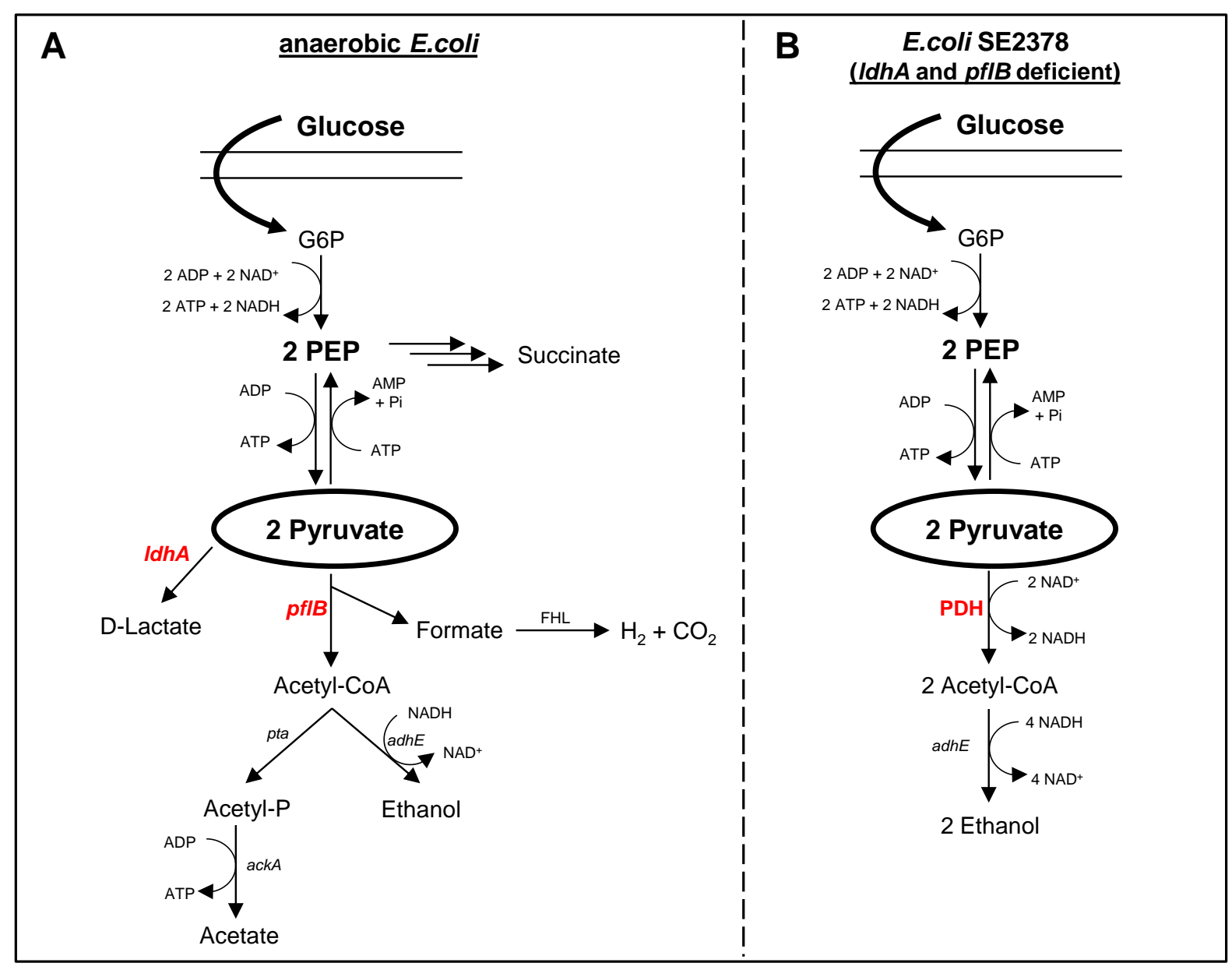

Figure 1.8 Comparison of mixed acid pathway for glucose fermentation.

(A) pathway in native $E$. coli and (B) in the $l d h A$ and $p f l B$ deficient $E$. coli strain SE2378.

G6P, glucose 6-phosphate; PEP, phosphoenolpyruvate; ldhA, lactate dehydrogenase; $p f l B$, pyruvate formate lyase; FHL, formate hydrogenlyase complex; pta, phosphotransacetylase; adhE, aldehydealcohol dehydrogenase; ackA, acetate kinase; PDH, pyruvate dehydrogenase complex. 


\subsection{The Relevance of Covalent Flavin-C4a-cysteinyl Adducts}

As described in the previous chapter (1.5) the FAD-C4a-cysteinyl adduct is a central intermediate during catalysis of E. coli lipoamide dehydrogenase. It is also found in other flavoenzymes belonging to the family of pyridine nucleotide-disulfide oxidoreductases, like glutathione reductase and mercuric reductase (Miller et al. 1990; Thorpe and Williams 1981). In these metabolic enzymes, the adduct ensures internal electron transfer from redox active disulfides to the flavin cofactor and vice versa. However, the function of FAD-C4a-cysteinyl adducts is not limited to electron transfer reactions, which involves thiol/disulfide chemistry. The FAD-C4a-cysteinyl adduct is also a central reaction intermediate in a class of flavoprotein light sensors: the light-oxygen-voltage (LOV) domains. These photosensor proteins can be found in plants, fungi, archaea and bacteria and are a subset of the PERARNT-SIM domain superfamily containing a noncovalently bound flavin cofactor (FMN or FAD) (Conrad et al. 2014). Using diverse output and effector domains, the photochemistry of LOV domains acts as signal transducer helping to mediate a variety of physiological functions like phototropism, chloroplast and leaf movements, and stomatal opening (Möglich et al. 2010). Blue light exposure induces the formation of a covalent FAD-C4a-cysteinyl adduct between the flavin and a conserved cysteine residue located at the active site (Figure 1.9). However, the structural rearrangements are not limited to the adduct site. Adduct formation requires the flavin N5 becoming protonated which causes a flipping of a conserved glutamine residue interacting with N5 (Figure 1.9B). This flipping was identified as a major event during signal transmission from the LOV domain to the output and effector domains (Raffelberg et al. 2011).

The LOV domain photocycle has been investigated thoroughly, but several key details are controversially discussed (Kennis and Groot 2007; Losi and Gärtner 2011; Zoltowski and Gardner 2011). In the dark state, the flavin is oxidized harboring an absorption maximum at $\sim 447 \mathrm{~nm}$ and is not attached to the protein backbone. The blue light induced covalent bond formation between flavin and the conserved cysteine leads to a hypsochromic shift resulting in a new absorption maximum at $\sim 390$ $\mathrm{nm}$ (Figure 1.9). The intermediate states prior to adduct formation are fast and comprise formation of an excited singlet state (S1) followed by an intersystem crossing yielding an excited triplet state (Alexandre et al. 2009; Pfeifer et al. 2009). The mechanism of the following adduct formation is a matter in controversy. On the one hand, the reaction is thought to proceed via an ionic intermediate, where a proton is transferred to flavin N5 before bond formation (Kennis and Groot 2007; Losi and Gärtner 2011, 2012; Zoltowski and Gardner 2011), on the other hand, further data suggest that the flavin cofactor is unprotonated in the excited triplet state immediately before reacting with the cysteine (Alexandre et al. 2009; Pfeifer et al. 2009). There is also some evidence for a neutral semiquinone intermediate between the triplet and the adduct state (Bauer et al. 2011) so that the identities of the intermediates prior to adduct formation are not quite clear. In addition, the proton source for N5 protonation has not been ultimately identified (Conrad et al. 2014). 
The major difference between FAD-C4a-cysteinyl adducts in LOV domain photoreceptor proteins and pyridine nucleotide-disulfide oxidoreductases is the lifetime of the adduct. In the oxidoreductases the intermediate is only transiently formed making a direct observation tedious work (Miller et al. 1990; Thorpe and Williams 1976b). On the contrary, LOV domains provide a surrounding which keeps the covalent adduct stable for at least $500 \mathrm{~ms}$, but the lifetime can even be expanded to hours (Conrad et al. 2014). The thermally driven recovery back to the dark state requires flavin N5 deprotonation, covalent bond breakage and protonation of the cysteine sulfur atom (Losi and Gärtner 2012). Generally, the rates of adduct decay depend on solvent access to the active site, the hydrogen bonding network around the flavin and its electronic environment (Conrad et al. 2014). However, it is suggested that the (de)protonation of N5 is the rate limiting step in the LOV photocycle (Kottke et al. 2003; Zoltowski, Vaccaro, and Crane 2009).

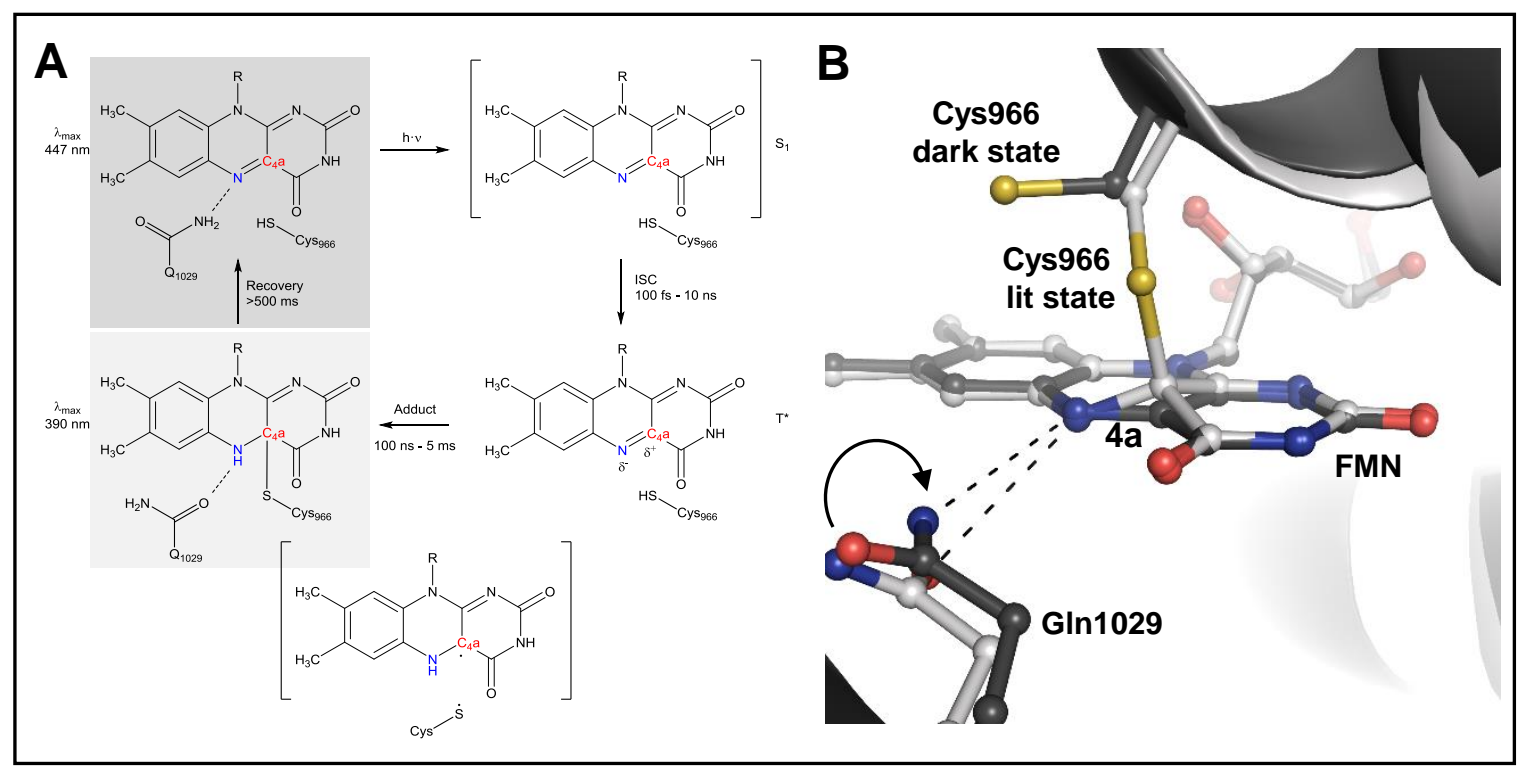

Figure 1.9 LOV domain photocycle and light-driven structural rearrangement.

(A) Light excites the dark-state FMN to a singlet state (S1) followed by an intersystem crossing (ISC) to an excited triplet state ( $\left.\mathrm{T}^{*}\right)$. $\mathrm{T}^{*}$ stimulates reaction of the C4a position (red) with an active site Cys residue to form a covalent adduct. Protonation of N5 (blue) during adduct formation leads to reorientation of a conserved glutamine residue (adapted from (Conrad et al. 2014). (B) Crystal structure of Adiantum LOV2 in dark (PDB: 1G28) and in lit state (PDB: 1JNU). Light induces covalent bond formation between Cys966 and C4a and the concomitant switch of Gln1029 from a H-bond donor in dark state to an H-bond acceptor towards N5 in lit state. 


\subsection{Motivation}

Lipoamide dehydrogenases have been extensively studied over the last decades and thus a detailed kinetic and structural knowledge about the mode of action of these enzymes could be gained. However, central reactions steps still lack both spectroscopic and structural information. The goal of this project is therefore to provide these information using the enzyme of E. coli (EcE3). Major emphasis will thereby be devoted to the spectroscopic and structural detection of the covalent FADC4a-cysteinyl adduct. The existence of this central intermediate has been predicted, but a direct evidence, especially in wild type enzymes, has not yet been given. Moreover, the reasons for its supposed transience are not fully understood (Argyrou et al. 2002). Structural data might help to explain the basis for the short-lived nature of the intermediate.

Spectroscopic analysis of the wild type enzyme and active-site mutants with conventional and stopped-flow UV-Vis spectroscopy shall be used to detect the FAD-C4a-cysteinyl adduct. Both single wavelength or photodiode array setups may help to identify active site residues which are crucial for adduct formation and/or the reported transience of the intermediate. The Investigations will be carried out from the forward and the reverse direction of the reaction cycle. NADH is a commonly used substrate which allows the investigation of different enzyme species harboring distinct spectroscopic signatures (Argyrou et al. 2002; Matthews and Williams 1976). However, the usage of NADH only allows the tracking of the oxidative half-reaction. Therefore, it is aimed to isolate an entire lipoyl domain from the $\mathrm{E} 2$ component of $E c \mathrm{PDHc}$ which may serve as an in vivo substrate for EcE3 after reduction of its lipoamide cofactor. This would allow the tracking of the reductive half-reaction with the aforementioned methods and further allow investigations in the field of protein-protein interactions.

Besides the characterization of the FAD-C4a-cysteinyl adduct a further intermediate state of the enzyme is of primary interest. The two-electron reduced state $\left(\mathrm{EH}_{2}\right)$ of $E c \mathrm{E} 3$ is the catalytically active species prior to generation of NADH. Detailed spectroscopic studies on this species have been carried out, but the ultimate factors stabilizing the anionic form of the cysteine facing the flavin cofactor remain unclear (Hopkins and Williams 1995a; Wilkinson and Williams 1979). An illumination of the thiolate stabilization by means of structural information is therefore obligate to further expand the knowledge about the $\mathrm{EH}_{2}$ state.

The three-dimensional structure of the lipoamide dehydrogenases enzyme revealed a striking similarity to the active site of LOV (light-oxygen-voltage) blue-light receptors (Chandrasekhar et al. 2013; Conrad et al. 2014; Mattevi et al. 1991). A common feature of the oxidoreductases and the lightsensing proteins is the formation of a covalent FAD-C4a-cysteinyl adduct during the respective reaction cycle (1.7). This study attempts the conversion of the metabolic EcE3 into a pseudo-LOV blue-light-sensing protein via structure-guided protein engineering which is capable of a light-driven 
C4a-cysteinyl adduct formation. If successful, a novel system for the light-driven generation of reducing equivalents could be obtained. An investigation of the photochemical response of reengineered $E c E 3$ variants using steady-state and time-resolved absorption spectroscopy.

Further attention will be dedicated to the dimer interface of $E c E 3$. It was demonstrated that the dimer interaction modulates the redox properties of the active site flavin cofactor in the A. vinelandii enzyme (Benen, Van Berkel, Veeger, et al. 1992). In addition, mutations in the same region reduced the sensitivity towards NADH inhibition of $E c E 3$ and concomitantly affected the entire fermentation profile of E. coli (Kim et al. 2008). Hence, the role of the interface is also of interest and will be investigated with respect to its ability to act as determinate for the flavin redox potential.

\section{Material and Methods}

\subsection{Material}

\subsubsection{Fine chemicals}

\section{Compound}

Acetic acid

Acrylamide

Agar

Agarose

Aldrithiol (4,4'-dithiopyridine)

Ammonium sulfate

Ammonium peroxodisulfate

Calcium chloride

Carbenicillin disodium salt

Coomassie Brillant blue G-250

Dithiothreitol (DTT)

Ethylenediaminotetraacetic acid (EDTA)

Ethanol

FAD

FMN

D-Glucose

\section{Supplier}

Carl Roth GmbH \& Co KG, Karlsruhe

Carl Roth GmbH \& Co KG, Karlsruhe

AppliChem GmbH, Darmstadt

AppliChem GmbH, Darmstadt

Sigma-Aldrich GmbH, Deisenhofen

AppliChem GmbH, Darmstadt

Carl Roth, Karlsruhe

Carl Roth GmbH \& Co KG, Karlsruhe

AppliChem, Darmstadt

AppliChem GmbH, Darmstadt

AppliChem GmbH, Darmstadt

AppliChem GmbH, Darmstadt

Carl Roth GmbH \& Co KG, Karlsruhe

AppliChem GmbH, Darmstadt

AppliChem GmbH, Darmstadt

AppliChem GmbH, Darmstadt 
Glycerol (99\%)

Glycine

Hydrochloric acid (37\%)

Imidazole

Kanamycin sulfate

Lipoamide

Lipoic acid

Lumichrom

Magnesium chloride, hexahydrate

Magnesium sulfate

$\beta$-Mercaptoethanol

$\mathrm{NAD}^{+} / \mathrm{NADH}$

Phenylmethylsulfonyl fluoride

Phosphoric acid (85\%)

Potassium chloride

Potassium dihydrogen phosphate

Potassium hydroxide

Polyethylene glycol (PEG) 400

Polyethylene glycol (PEG) 4000

Riboflavin

Sodium chloride

$\mathrm{N}, \mathrm{N}, \mathrm{N}^{\prime}, \mathrm{N}^{\prime}$-Tetramethylethylendiamin

Thiamine hydrochloride

Tris(2-carboxyethyl)phosphine hydrochloride

Tryptone

Yeast extract

\subsubsection{Devices}

ACQUITY UPLC ${ }^{\mathrm{TM}}$ System

Äkta Prime Plus

Äkta Purifier

Agilent 6540 UH Accurate-Mass-Q-TOF MS

Agilent 8453 UV-visible spectrophotometer

Autoclave

Balance Kern EW

Balance Kern ABJ
AppliChem GmbH, Darmstadt

Carl Roth GmbH \& Co KG, Karlsruhe

Carl Roth GmbH \& Co KG, Karlsruhe

AppliChem GmbH, Darmstadt

Carl Roth GmbH \& Co KG, Karlsruhe

Sigma-Aldrich GmbH, Deisenhofen

Sigma-Aldrich GmbH, Deisenhofen

Sigma-Aldrich GmbH, Deisenhofen

Carl Roth GmbH \& Co KG, Karlsruhe

Carl Roth GmbH \& Co KG, Karlsruhe

Carl Roth GmbH \& Co KG, Karlsruhe

AppliChem GmbH, Darmstadt

AppliChem GmbH, Darmstadt

Carl Roth GmbH \& Co KG, Karlsruhe

Carl Roth GmbH \& Co KG, Karlsruhe

Carl Roth GmbH \& Co KG, Karlsruhe

AppliChem GmbH, Darmstadt

Fluka Chemie AG, Buchs, Switzerland

Carl Roth GmbH \& Co KG, Karlsruhe

Sigma-Aldrich GmbH, Deisenhofen

AppliChem GmbH, Darmstadt

Carl Roth GmbH \& Co KG, Karlsruhe

AppliChem GmbH, Darmstadt

Sigma-Aldrich Chemie, Deisenhofen

AppliChem GmbH, Darmstadt

AppliChem GmbH, Darmstadt

Waters Corporation, Milford, USA

GE Healthcare, Munich

GE Healthcare, Munich

Agilent Technologies, Böblingen

Agilent Technologies, Santa Clara, USA

Zirbus, Bad Grund

Kern, Balingen-Frommern

Kern, Balingen-Frommern 
Centrifuge Avanti J-20 XPIJA-20

Centrifuge Avanti J-30 I

Centrifuge Hettich Universal 320 R

Conducting meter FiveEasy

$\underline{E P R}$

Spectrometer: Lab-built X-Band spectrometer with:

Microwave bridge ER 041 MR

Microwave controller ER 048 R

Magnet power supply ER $081 \mathrm{~S}$

Field controller BH 15

Cavity resonator ER 4122 SHQ E

SR 810 lock-In detector

53181A frequency counter

ESR 910 cryostat

ITC503 temperature controller

Sample tubes: $3.0 \mathrm{~mm} / 3.9 \mathrm{~mm}$ diameter quartz

Frac-920 Fraction Collector

Incubator Unitron

Incubator CrystalMotion

iTC $_{200}$ MicroCalorimeter

LCT Premier ${ }^{\mathrm{TM}}$ ESI-TOF-MS

MALDI-TOF Reflex III

Carrier MTP 384 polished steel

Microfluidizer $110 \mathrm{~S}$

pH meter FiveEasy

Pipette robot Phoenix RE

Rotor JA-30.50 Ti

Rotor JLA 8.1000

SDS gel electrophoresis SE250

UPLC eLambda $800 \mathrm{~nm}$

$\mathrm{X}$-ray detector mar345

$\mathrm{X}$-ray diffractometer RU-H3R

Spectrometers

Chirascan (CD)

Stopped-flow system SX.20 MV

NanoDrop2000

V-650 (UV/Vis)
Beckmann Coulter, Krefeld

Beckmann Coulter, Krefeld

Hettich, Tuttlingen

Mettler-Toledo, Giessen

Bruker, Rheinstetten

Bruker, Rheinstetten

Bruker, Rheinstetten

Bruker, Rheinstetten

Bruker, Rheinstetten

Stanford Research Systems, Sunnyvale, USA

Agilent Technologies, Santa Clara, USA

Oxford Instruments, East Grinstead, UK

Oxford Instruments, East Grinstead, UK

Qsil, Ilmenau

GE Healthcare, Munich

HT Infors, Bottmingen

Rigaku, Kent, England

Microcal, Northampton, USA

Waters Corporation, Milford, USA

Bruker Daltonics, Bremen

Bruker Daltonics, Bremen

Microfluidics, Newton, USA

Mettler-Toledo, Giessen

Art Robbins Instruments, USA

Beckmann Coulter, Krefeld

Beckmann Coulter, Krefeld

Hoefer, Holliston, USA

Waters Corporation, Milford, USA

Marresearch, Norderstedt

Rigaku/MSC, Japan

Applied Photophysics, GB

Applied Photophysics Ltd., UK

Thermo Scientific, Schwerte

JASCO, Gross-Umstadt 
Stereomicroscope SZ 60

Thermocycler TProfessional

Vortex-Genie 2

Water bath Isotemp 202

Commodities

0.2 and $0.45 \mu \mathrm{m}$ Filter

Circular cover slides, $18 \mathrm{~mm}$

Crystallization plates (24 well)

UV- and CD-cuvettes

Spin- $\mathrm{X}^{\circledR}$ UF concentrator

ZipTip $_{\mathrm{C} 18}$ Pipette Tips

\subsubsection{Chromatography Columns}

HiPrep $^{\text {TM }}$ 26/10 Desalting

HisTrap ${ }^{\text {TM }}$ Ni-NTA-Sepharose $(25 \mathrm{ml})$

Superdex ${ }^{\text {TM }} 200$ XK 16/600

\subsubsection{Molecular Weight Marker}

Unstained Protein Molecular Weight Marker

Peptide Calibration Standard

\subsubsection{Kit-Systems}

NucleoSpin ${ }^{\circledR}$ Extract II

NucleoSpin ${ }^{\circledR}$ Plasmid

QuikChange site directed mutagenesis kit

\subsubsection{Materials for Molecular Biology}

6X DNA Loading Dye

dNTPs (Mix $10 \mathrm{mM}$ )

FastAPTM Thermosensitive Alkaline Phosphatase

GeneRuler $^{\mathrm{TM}} 1$ kb DNA Ladder

HF-buffer
Olympus, Japan

Biometra, Göttingen

Scientific Industries, New York, USA

Fisher Scientific, Schwerte

Sartorius AG, Göttingen

Jena Bioscience, Jena

Costar Corning, Inc., USA

Hellma, Müllheim

Corning, Corning, USA

Millipore, Billerica, USA

GE Healthcare, Munich

GE Healthcare, Munich

GE Healthcare, Munich

Thermo Scientific, Schwerte

Bruker Daltonics, Bremen
Thermo Scientific, Schwerte

Thermo Scientific, Schwerte

Thermo Scientific, Schwerte

Thermo Scientific, Schwerte

Thermo Scientific, Schwerte 
Magnesium chloride (25 mM)

Phusion Polymerase

T4 DNA Ligase

T4 DNA Ligase Buffer

Taq Buffer (10-fold)

Taq Polymerase
Thermo Scientific, Schwerte

Thermo Scientific, Schwerte

Thermo Scientific, Schwerte

Thermo Scientific, Schwerte

Thermo Scientific, Schwerte

Thermo Scientific, Schwerte

\subsubsection{Restriction Endonucleases}

NdeI

XhoI

DpnI
Thermo Scientific, Schwerte

Thermo Scientific, Schwerte

Thermo Scientific, Schwerte

\subsubsection{Bacterial Strains}

Strain

E. coli BL21 (DE3)

E. coli BL21 StarTM (DE3)

E. coli DH5 $\alpha^{\mathrm{TM}}$
Genotype

$\mathrm{F}^{-}$ompT hsdS $S_{B}\left(\mathrm{r}_{\mathrm{B}}{ }^{-} \mathrm{m}_{\mathrm{B}}{ }^{-}\right)$gal dcm (DE3)

$\mathrm{F}^{-}$ompT hsd $S_{B}\left(\mathrm{r}_{\mathrm{B}}{ }^{-} \mathrm{m}_{\mathrm{B}}{ }^{-}\right)$gal dcm rne131 (DE3)

$\mathrm{F}^{-}$Ф80lacZ $\Delta$ M15 $\Delta$ (lacZYA-argF) U169

rec $\mathrm{A} 1$ end $\mathrm{A} 1$ hsd $\mathrm{R} 17\left(\mathrm{r}_{\mathrm{K}}{ }^{-}, \mathrm{m}_{\mathrm{K}}{ }^{+}\right)$pho $\mathrm{A} \sup \mathrm{E} 44$

$\lambda^{-}$thi 1 gyr 96 relA1
Reference

Invitrogen

Invitrogen

Invitrogen

\subsubsection{Vectors}

pET15b-EcE2

pET28a

pGS523

pET-SUMO
GENEART, Regensburg

Merck KGaA, Darmstadt

John R. Guest, University of Sheffield

Invitrogen, Darmstadt

\subsubsection{Enzymes}

DNase I

Lysozyme

SUMO-Protease

Thrombin

Trypsin
AppliChem, Darmstadt

AppliChem, Darmstadt

provided by Dr. Stefan Lüdtke

Sigma-Aldrich GmbH, Deisenhofen

Promega, Mannheim 


\subsubsection{Crystallization Screens}

Ammonium sulfate

JCSG

JBScreen Nuc-Pro HTS

Morpheus screen

Natrix HT

PGA-LM HT-96

ProPlex

\subsubsection{Software}

CCP4 suite

Chromas 1.45

Crystallography \& NMR System (CNS)

ESPript 3.0

ExPasy, ProtParam

FlexAnalysis

Gene runner V.3.05

Origin-7

PeptideCutter

Phenix suite

PYMOL

Mass Hunter Qualitative Analysis software (B.06.00)

MassLynx V4.1 SCN779

METLIN

MicroCal ITC-ORIGIN Analysis Software

MOLPROBITY

Multalin

SigmaPlot Version 11.0
Qiagen, Hilden

Molecular Dimensions, Suffolk, UK

Jena Bioscience, Jena

Fabrice Gorrec, Cambridge, UK

Hampton Research, Aliso Viejo, USA

Molecular Dimensions, Suffolk, UK

Molecular Dimensions, Suffolk, UK

Winn et al., 2011

McCarthy, C., Griffith University,

Australia

(Brunger 2007)

http://espript.ibcp.fr/ESPript/ESPript

/index.php

http://web.expasy.org/protparam/

Bruker Daltonics, Bremen

Hastings Software, Inc.

OriginLab Corporation, USA

http://web.expasy.org/peptide_cutter/

Adams et al., 2010

Schrödinger, LLC

Agilent Technologies, Böblingen, Germany

Waters Corporation, Milford, USA

https://metlin.scripps.edu/index.php

Microcal, Northampton, USA

http://molprobity.biochem.duke.edu/

Corpet 2010

Systat Software, Inc. 


\subsection{Methods}

\subsubsection{Molecular Biology}

The gene of EcE3 was amplified from pGS523 (Machado, Clark, and Guest 1992), kindly provided from Prof. John Guest (University of Sheffield), and cloned into the pET28a expression vector using the XhoI and NdeI restriction sites. This expression construct encodes for $E c \mathrm{E} 3$ with an N-terminal hexa-histidine tag followed by a thrombin cleavage site. The N-terminal tag extends EcE3 by 20 amino acids, of which three will remain after thrombin cleavage. The amino acid sequences of all purified proteins are shown in the appendix (5.1).

The EcLip3 gene encodes for amino acids $201-286$ of the E. coli E2 component and thus represents the isolated innermost lipoyl domain. The EcLip3 gene was amplified from pET15b-EcE2 and cloned into the target vector pET-SUMO using the TA cloning ${ }^{\circledR}$ strategy according to the Champion ${ }^{\mathrm{TM}} \mathrm{pET}$ SUMO Protein Expression System manual.

To introduce specific amino acid exchanges within the sequence of $E c E 3$ site-directed mutagenesis was used. Variants were generated according to the QuikChange-Kit (Stratagene, USA) with oligonucleotides containing the mutated codons. Double variants were generated starting with a single mutated plasmid as template. All used template amplification and mutagenesis primer pairs are listed in Table 2.1.

Table 2.1 List of used primer pairs (Synthesized by Sigma-Aldrich, Taufkirchen). Restriction sites are underlined, unspecific overhangs are uncapitalized, mutations sites are shown in bold face type.

\begin{tabular}{|c|c|}
\hline \multicolumn{2}{|c|}{ Template amplification primer pairs } \\
\hline Primername & Nucleotide sequence (5'-3') \\
\hline E3_NdeI_2for & accatgcatatgAGTACTGAAATCAAAACTCAGGTCGTGG \\
\hline E3_Xho_rev & tagcctctcgagttaCTTCTTCTTCGCTTTCGGGTTCGGC \\
\hline Lip3-201for & GCACCAGCGGCTGGCGTG \\
\hline Lip3-286for & tcattaCGCAGGCGCTGCGCCTTC \\
\hline \multicolumn{2}{|c|}{ Mutagenesis primer pairs } \\
\hline Primername & Nucleotide sequence (5'-3') \\
\hline E3_Y19F_for & GGCCCCGCAGGTTTCTCCGCTGCCTTCC \\
\hline E3_Y19F_rev & GGAAGGCAGCGGAGAAACCTGCGGGGCC \\
\hline
\end{tabular}




\begin{tabular}{|c|c|}
\hline E3_C45A_for & CCTTGGCGGTGTTGCCCTGAACGTCGGC \\
\hline E3_C45A_rev & GCCGACGTTCAGGGCAACACCGCCAAGG \\
\hline E3_C45S_for & CCTTGGCGGTGTTTCCCTGAACGTCGGC \\
\hline E3_C45S_rev & GCCGACGTTCAGGGAAACACCGCCAAGG \\
\hline E3_C50A_for & GCCTGAACGTCGGCGCTATCCCTTCTAAAGCACTGC \\
\hline E3_C50A_rev & GCAGTGCTTTAGAAGGGATAGCGCCGACGTTCAGGC \\
\hline E3_C50S_for & GCCTGAACGTCGGCTCTATCCCTTCTAAAGCACTGC \\
\hline E3_C50S_rev & GCAGTGCTTTAGAAGGGATAGAGCCGACGTTCAGGC \\
\hline E3_C50M_for & GCCTGAACGTCGGCATGATCCCTTCTCAAGC \\
\hline E3_C50M_rev & GCTTGAGAAGGGATCATGCCGACGTTCAGGC \\
\hline E3_K54Q_for & GGCTGTATCCCTTCTCAAGCACTGCTGCACG \\
\hline E3_K54Q_rev & CGTGCAGCAGTGCTTGAGAAGGGATACAGCC \\
\hline E3_E189Q_for & GGTATCATCGGTCTGCAAATGGGCACCGTTTACC \\
\hline E3_E189Q_rev & GGTAAACGGTGCCCATTTGCAGACCGATGATACC \\
\hline E3_E354K_for & CCATCGCCTATACCAAACCAGAAGTTGCATGG \\
\hline E3_E354K_for & CCATGCAACTTCTGGTTTGGTATAGGCGATGG \\
\hline E3_R386A_for & GGGCTGCTTCTGGTGCTGCTATCGCTTCCG \\
\hline E3_R386A_rev & CGGAAGCGATAGCAGCACCAGAAGCAGCCC \\
\hline E3_H445A_for & CCATCCACGCGGCCCCGACTCTGCACG \\
\hline E3_H445A_rev & CGTGCAGAGTCGGGGCCGCGTGGATGG \\
\hline E3_E450Q_for & CCCGACTCTGCACCAGTCTGTGGGCCTGG \\
\hline E3_E450Q_rev & CCAGGCCCACAGACTGGTGCAGAGTCGGG \\
\hline
\end{tabular}




\subsubsection{Concentration Determination of DNA}

DNA concentrations were determined spectroscopically using a NanoDrop2000 by measuring the absorption at $260 \mathrm{~nm}$. The following correlation was used to determine the concentrations: $1 \mathrm{~A}_{260}=$ $50 \mu \mathrm{g} / \mathrm{ml}$ doubled stranded DNA.

\subsubsection{Agarose Gelelectrophoresis}

Agarose gelelectrophoresis was used for analysis of DNA fragments after PCR reactions. Samples were supplemented with DNA loading dye (6 x DNA loading dye: $30 \%$ (v/v) glycerol, $0.25 \%$ (w/v) bromophenol blue) and were separated using a horizontal electrophoresis. Gels typically contained $1 \%$ agarose and $1 \times$ TAE buffer $(40 \mathrm{mM}$ Tris, $1 \mathrm{mM}$ EDTA, $20 \mathrm{mM}$ acetate, $\mathrm{pH} 8.5)$. For visualization the gel was transferred into a solution containing $2 \mu \mathrm{g} / \mathrm{ml}$ ethidium bromide and incubated for 5-10 min prior to detection under UV light in a gel documentation system.

\subsubsection{Polymerase Chain Reaction (PCR)}

PCR was used for both amplification of gene fragments and for site directed mutagenesis. A standard reaction of a total volume of $50 \mu 1$ contained $10 \mu \mathrm{l}$ x HF buffer, $1 \mu \mathrm{dNTP}$ mix (10 mM), 5-50 ng template DNA, 25 pmol sense and antisense primer, and $0.5 \mu$ l Phusion DNA Polymerase ( $2 \mathrm{U} / \mu \mathrm{l})$. The PCR protocol included an initial denaturation step (step $1 ; 95^{\circ} \mathrm{C}, 1 \mathrm{~min}$ ), another denaturation step (step $2 ; 95^{\circ} \mathrm{C}, 30 \mathrm{~s}$ ), a primer annealing step (step 3; 50-60 ${ }^{\circ} \mathrm{C}, 30 \mathrm{~s}$ ), an elongation step (step 4; $72{ }^{\circ} \mathrm{C}, 20 \mathrm{~s}$ per kb product), and an end elongation (step 5; $72{ }^{\circ} \mathrm{C}, 7 \mathrm{~min}$ ). Steps $2-4$ were repeated 25 times. If site-directed mutagenesis PCR was carried out the mixture was supplemented with DpnI after the PCR to digest template DNA (see QuikChange site-directed mutagenesis kit).

\subsubsection{DNA Sequencing}

Correctness of was confirmed using two commercially available sequencing services from SeqlabMicrosynth GmbH, Göttingen and GATC Biotech AG, Cologne.

\subsubsection{Plasmid Transformation}

Transfer of plasmid DNA into different E. coli strains was performed using the following standard protocols chemical-competent cells were prepared according to Inoue et al. (Inoue, Nojima, and Okayama 1990). Competent cells were stored at $-80{ }^{\circ} \mathrm{C}$. In a standard transformation, $1 \mu \mathrm{l}$ of plasmid DNA was mixed with $50 \mu \mathrm{l}$ of chemo-competent E. coli cells followed by an incubation step for 20 min on ice. For DNA transformation the cells were heat-shocked for $45 \mathrm{~s}$ at $42{ }^{\circ} \mathrm{C}$ and subsequently cooled on ice for $2 \mathrm{~min}$. Afterwards, $500 \mu \mathrm{l}$ of SOC medium ( $2 \%(\mathrm{w} / \mathrm{v})$ tryptone, $0.5 \%(\mathrm{w} / \mathrm{v})$ yeast 
extract, $0.05 \%$ (w/v) NaCl, $2.5 \mathrm{mM} \mathrm{KCl}, 10 \mathrm{mM} \mathrm{MgCl}_{2}, 2 \%$ (w/v) glucose) was added and the cells were incubated for $45 \mathrm{~min}$ at $37{ }^{\circ} \mathrm{C}$ under shaking. Transformed cells were selected on LB-agar plates supplemented with the appropriate antibiotic. Final antibiotic concentrations in media were $50 \mu \mathrm{g} / \mathrm{ml}$ kanamycin, or $50 \mu \mathrm{g} / \mathrm{ml}$ carbenicillin.

\subsubsection{Protein Chemistry}

\subsubsection{Sodium Dodecyl Sulfate - Polyacrylamide Gel Electrophoresis (SDS-PAGE)}

All proteins were analyzed by SDS-PAGE based on a protocol of Laemmli and Weber (Laemmli 1970). The stacking gel consisted of $5 \%$ acrylamide, $125 \mathrm{mM}$ Tris ( $\mathrm{pH} 6.8$ ) polymerized with $0.3 \%$ w/v APS and $0.03 \%$ TEMED, whereas the separating gel contained 12-15\% acrylamide, $375 \mathrm{mM}$ Tris ( $\mathrm{pH} 8.8$ ) polymerized with $0.3 \%$ APS and $0.3 \%$ TEMED. Prior to loading protein samples were mixed with SDS loading buffer (4\% (w/v) glycerol, $80 \mathrm{mM}$ SDS, $150 \mathrm{mM}$ Tris, $\mathrm{pH}$ 6.8) and heated to $95{ }^{\circ} \mathrm{C}$ for $5 \mathrm{~min}$. Gels were usually run at $1 \mathrm{~mA} / \mathrm{cm}^{2}$ in SDS running buffer $(25 \mathrm{mM}$ Tris, $200 \mathrm{mM}$ glycine und $3.5 \mathrm{mM}$ SDS). After protein separation was completed the gels were stained with $0.25 \%$ (w/v) Coomassie Brilliant Blue in $30 \%$ ethanol and $6 \%$ acetic acid for $30 \mathrm{~min}$ and destained in $30 \%$ ethanol and $10 \%$ acetic acid. For short-term storage gels were covered with $\mathrm{ddH}_{2} \mathrm{O}$ and kept at room temperature.

\subsubsection{Protein Expression and Purification of $E c E 3$ and multiple Variants from pGS523}

Expression and purification of $E c$ E3 was basically described by LINDSEY (Lindsay et al. 2000). The expression plasmid pGS523 (encoding the entire EcPDHc) was transformed into chemical competent E. coli $\mathrm{BL} 21^{\mathrm{TM}}$ cells. An overnight preculture grown at $37^{\circ} \mathrm{C}$ in Luria-Bertani medium containing $50 \mu \mathrm{g} / \mathrm{mL}$ carbenicillin was used to inoculate a main culture of the same medium to an $\mathrm{OD}_{600}$ of 0.1 . Cells were grown at $37^{\circ} \mathrm{C}$ to an $\mathrm{OD}_{600}$ of 0.8 and expression was started by addition of $50 \mu \mathrm{M}$ IPTG and $100 \mu \mathrm{M}$ FMN. After four hours of expression the cells were harvested by centrifugation at $5000 \mathrm{x}$ g, resuspended in lysis buffer supplemented with lysozyme (tip of a spatula) and DNase I (tip of a spatula). Cell disruption was performed using a Microfluidizer $110 \mathrm{~S}$ followed by removal of the cell debris by centrifugation $\left(74,000 \mathrm{x}\right.$ g for $30 \mathrm{~min}$ at $\left.4{ }^{\circ} \mathrm{C}\right)$. The supernatant was subjected to a heat precipitation at $65{ }^{\circ} \mathrm{C}$ for 10 minutes using a water bath. Precipitated proteins were removed by centrifugation $\left(74,000 \mathrm{x}\right.$ g for $30 \mathrm{~min}$ at $\left.4{ }^{\circ} \mathrm{C}\right)$. Subsequently the clarified supernatant was loaded onto a HiLoad $^{\mathrm{TM}}$ 26/10 Q Sepharose ${ }^{\mathrm{TM}} \mathrm{HP}$ anion exchange column equilibrated in buffer A. EcE3 was eluted using a linear gradient of high salt buffer (buffer B) over two CV. Fractions containing $E c E 3$ were combined and desalted using a HiPrep ${ }^{\mathrm{TM}}$ 26/10 Desalting column equilibrated in buffer C. 
Table 2.2 Overview of buffers used for purification of $E c E 3$ from pGS523

\section{Lysis buffer}

Imidazole $20 \mathrm{mM}$

$\mathrm{MgCl}_{2} \quad 1 \mathrm{mM}$

PSMSF $1 \mathrm{mM}$

pH 6.5
Buffer A

Buffer B

Buffer C

\subsubsection{Protein Expression and Purification of $E c E 3$ and multiple Variants from pET28a}

For expression, pET28aEcE3 was transformed into chemical competent E.coli BL21 $\mathrm{Star}^{\mathrm{TM}}$ cells. An overnight preculture grown at $37{ }^{\circ} \mathrm{C}$ in Luria-Bertani medium containing $50 \mu \mathrm{g} / \mathrm{mL}$ kanamycin was used to inoculate a main culture of the same medium to an $\mathrm{OD}_{600}$ of 0.1 . Cells were grown at $37{ }^{\circ} \mathrm{C}$ to an $\mathrm{OD}_{600}$ of 0.8 and expression was started by addition of $500 \mu \mathrm{M}$ IPTG. After four hours of expression the cells were harvested by centrifugation at $5000 \mathrm{xg}$, resuspended in lysis buffer supplemented with lysozyme (tip of a spatula), DNase I (tip of a spatula). Cell disruption was performed using a Microfluidizer $110 \mathrm{~S}$ followed by removal of the cell debris by centrifugation $\left(74,000 \mathrm{xg}\right.$ for $30 \mathrm{~min}$ at $\left.4{ }^{\circ} \mathrm{C}\right)$. The supernatant was loaded onto a Ni-NTA FF Sepharose FF equilibrated in buffer $\mathrm{A}$ and $\mathrm{N}$-terminal $\mathrm{His}_{6}{ }_{6}$ tagged- $E c \mathrm{E} 3$ was eluted using high imidazole buffer $\mathrm{B}$ in a single step. Appropriate fractions were pooled, desalted with low imidazole buffer and the His-tag was removed by thrombin cleavage in the presence of $5 \mathrm{mM} \mathrm{CaCl}_{2}$ in the cold room for 12 hours. Cleaved His-tags were trapped by a second cycle of IMAC. Subsequently, the flow through was concentrated and loaded onto a HiLoad 16/600 S200 gel filtration column equilibrated in buffer C. If the $\mathrm{His}_{6}$-tag remained on $E c \mathrm{E} 3$ the protein was directly desalted against buffer $\mathrm{C}$ after the Ni-NTA column. All pET28aEcE3 variants were expressed and purified to homogeneity according to the method for the wild type.

Table 2.3 Overview of buffers used for purification of $E c E 3$ from pET28a

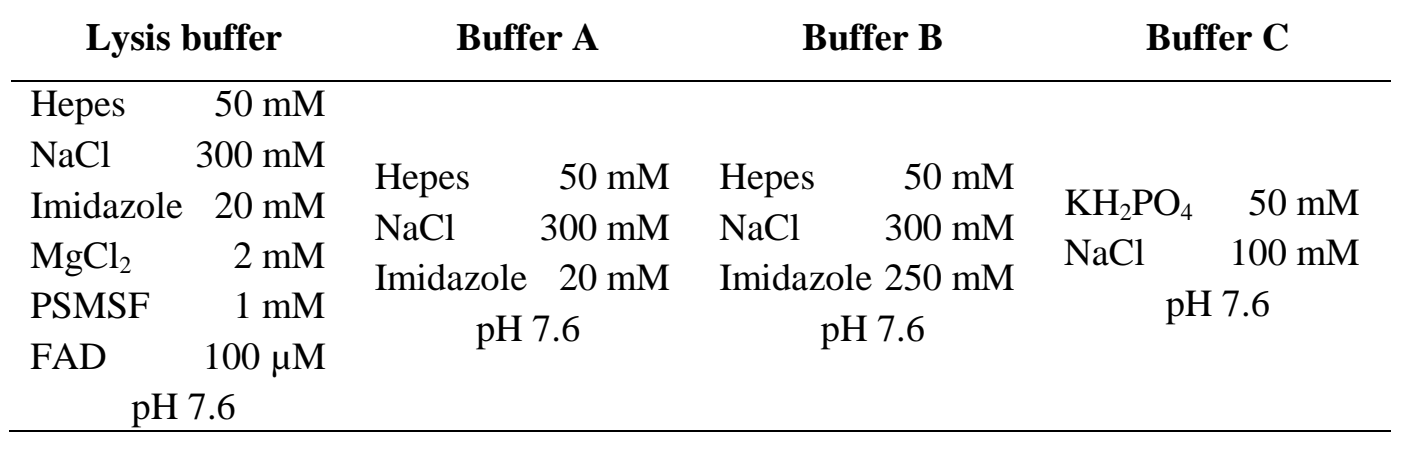




\subsubsection{Expression and Purification of EcLip3}

For expression pET-SUMO-EcLip3 was transformed into chemical competent E.coli BL21 Star ${ }^{\mathrm{TM}}$ cells. An overnight preculture grown at $37^{\circ} \mathrm{C}$ in Luria-Bertani medium containing $50 \mu \mathrm{g} / \mathrm{mL}$ kanamycin was used to inoculate a main culture of the same medium to an $\mathrm{OD}_{600}$ of 0.1 . In addition, the main culture contained $1 \%(\mathrm{w} / \mathrm{v})$ glucose to prevent basal expression. Cells were grown at $37{ }^{\circ} \mathrm{C}$ to an $\mathrm{OD}_{600}$ of 0.8 and expression was started by addition of $500 \mu \mathrm{M}$ IPTG. To ensure a sufficient degree of lipoylation expression was carried out in chicane flasks and in the presence of $0.2 \mathrm{mM}$ lipoic acid. After four hours of expression the cells were harvested by centrifugation at $5000 \mathrm{xg}$, resuspended in lysis buffer supplemented with lysozyme (tip of a spatula), DNase I (tip of a spatula). Cell disruption was performed using a Microfluidizer $110 \mathrm{~S}$ followed by removal of the cell debris by centrifugation $\left(74,000 \mathrm{xg}\right.$ for $30 \mathrm{~min}$ at $\left.4{ }^{\circ} \mathrm{C}\right)$. The supernatant was loaded onto a Ni-NTA FF Sepharose FF equilibrated in buffer A and N-terminal His ${ }_{6}$ tagged-SUMO-EcLip3 was eluted using high imidazole buffer $\mathrm{B}$ in a single step. Appropriate fractions were pooled, desalted with low imidazole buffer and the SUMO part was removed by cleavage with SUMO protease $(16.1 \mu \mathrm{M}$ in $50 \%$ (v/v) glycerol, $25 \mathrm{mM}$ Tris- $\mathrm{HCl} \mathrm{pH} 8.0,250 \mathrm{mM} \mathrm{NaCl}, 0.5 \mathrm{mM}$ dithiothreitol, $0.1 \%$ (v/v) IGEPAL) in a 1:2000 molar ratio at $4{ }^{\circ} \mathrm{C}$ for 12 hours. Cleaved SUMO protein, protease and residual uncleaved fusion protein were trapped by a second cycle of IMAC. Subsequently, the flow through was desalted using a HiPrep ${ }^{\text {TM }}$ 26/10 Desalting column equilibrated in buffer C.

Table 2.4 Overview of buffers used for purification of EcLip3

Lysis buffer

Buffer A

Buffer B

Buffer C

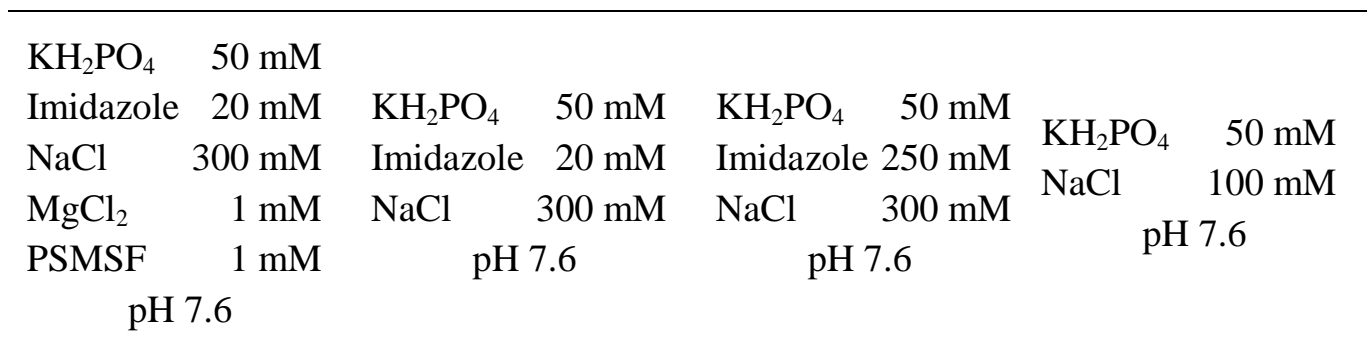




\subsubsection{Generation of dihydro-EcLip3 $\left(E c \mathrm{Lip3}_{2}\right)$}

Purified EcLip3 was transferred in its dihydro form by reduction of the lipoamide cofactor with a 5fold molar excess of TCEP for 5 minutes at room temperature. The reducing agent was removed from the protein by applying the reaction mixture to a HiPrep ${ }^{\mathrm{TM}}$ 26/10 Desalting column equilibrated in buffer C.

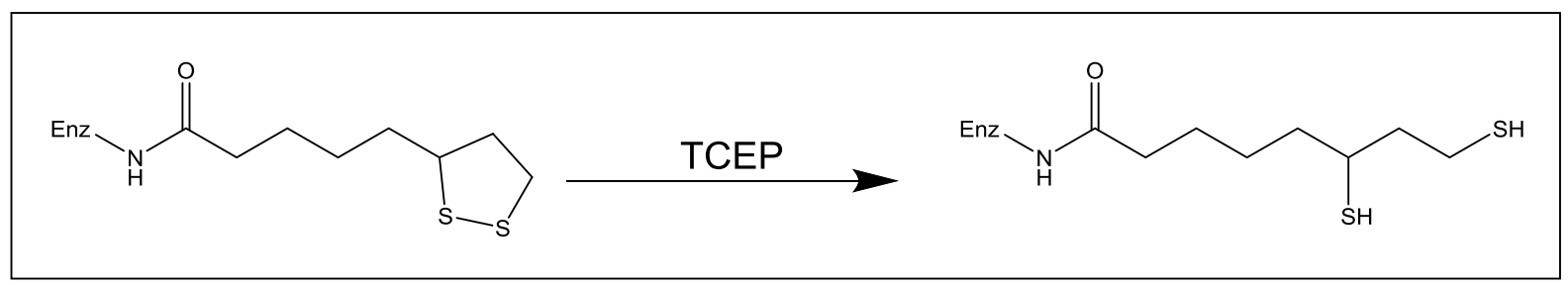

Figure 2.1 Simplified reaction scheme of the EcLip3 reduction by TCEP.

Reduction of the dithiolane moiety by TCEP leads to a ring opening and a concomitant loss of the chromophoric nature of the cystine at $333 \mathrm{~nm}$ (Levitch 1958; Seifert 2010).

\subsubsection{Concentration Determination of Proteins}

The concentration of proteins harboring a chromophoric cofactor was determined spectroscopically at the appropriate wavelength. EcE3 enzyme bound FAD was determined at $455 \mathrm{~nm}$ with an $\varepsilon=11,300 \mathrm{M}^{-1} \mathrm{~cm}^{-1}$ (Wilkinson and Williams 1981). The concentration of EcLip3 was determined at $333 \mathrm{~nm}$ with an $\varepsilon=150 \mathrm{M}^{-1} \mathrm{~cm}^{-1}$ due to the absorption of the dithiolane moiety of the lipoamide cofactor (Levitch 1958).

\subsubsection{Concentration Determination of dihydro-EcLip3}

The concentration of $E c \mathrm{Lip}_{3} \mathrm{H}_{2}$ was determined from the reduction of excess 4,4'-dithiopyridine to generate 4-thiopyridone ( $324 \mathrm{~nm}, \varepsilon=21.400 \mathrm{M}^{-1} \mathrm{~cm}^{-1}$ ) (Argyrou et al. 2003). Note that one molecule EcLip3 harbors two thiol groups.

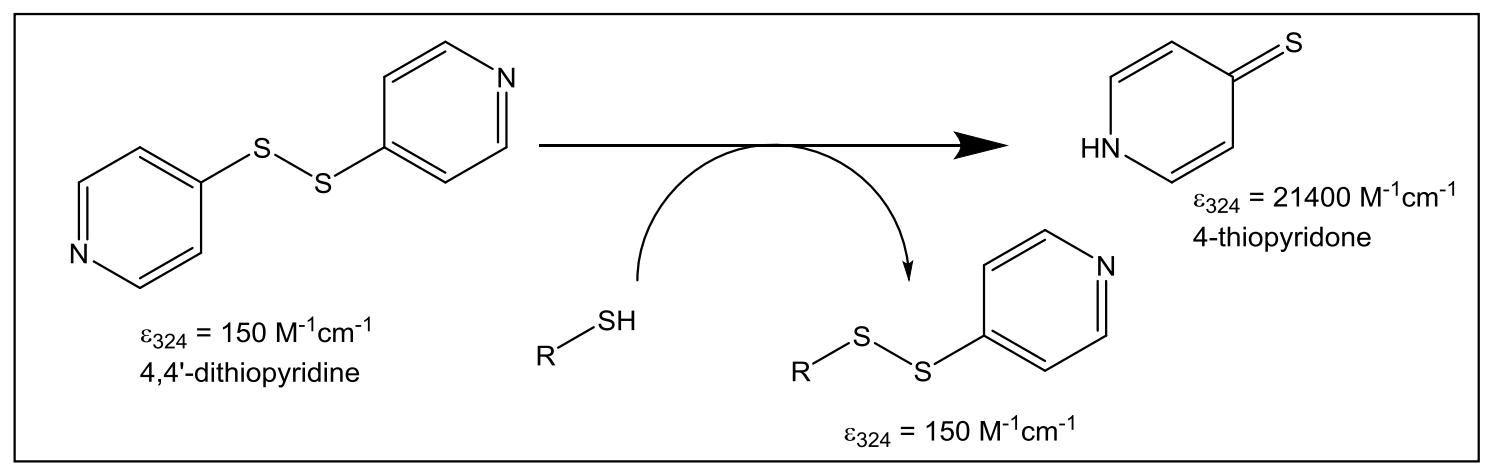

Figure 2.2 Reaction scheme of the concentration determination of free thiol groups. 


\subsubsection{Determination of Extinction Coefficients of Multiple Variants}

The extinction coefficient of protein-bound FAD was determined by releasing the FAD from the protein followed by quantitating the free FAD (Hopkins and Williams 1995b). The absorbance at the flavin peak was measured at concentrations between $8-180 \mu \mathrm{M}$ in $50 \mathrm{mM} \mathrm{KPP}, \mathrm{pH} 7.6$ and $100 \mathrm{mM}$ $\mathrm{NaCl}$. Guanidinium chloride (8 M, in $10 \mathrm{mM} \mathrm{KPP} \mathrm{pH} \mathrm{7.6)} \mathrm{was} \mathrm{added} \mathrm{to} \mathrm{a} \mathrm{final} \mathrm{concentration} \mathrm{of} 4.5 \mathrm{M}$ at room temperature. Samples supplemented with guanidinium chloride were centrifuged for $30 \mathrm{~min}$ at $21000 \mathrm{x} \mathrm{g}$. The extinction coefficient of free FAD is enhanced in the presence of $4.5 \mathrm{M}$ guanidinium chloride to a value of $11800 \mathrm{M}^{-1} \mathrm{~cm}^{-1}$ and thus was used in the calculations to determine the extinction coefficients of enzyme-bound FAD.

Table 2.5 Molar extinction coefficients of $E c E 3$ and respective variants.

\begin{tabular}{|c|c|c|}
\hline Enzyme variant & Spectroscopic characteristic & Reference \\
\hline wild type & $\mathrm{E}_{455 \mathrm{~nm}}=11300 \mathrm{M}^{-1} \mathrm{~cm}^{-1}$ & Wilkinson \& Williams 1981 \\
\hline Y19F & $\mathrm{E}_{455 \mathrm{~nm}}=11300 \mathrm{M}^{-1} \mathrm{~cm}^{-1}$ & This work \\
\hline $\mathrm{C} 45 \mathrm{~A}$ & $\mathrm{E}_{448 \mathrm{~nm}}=11940 \mathrm{M}^{-1} \mathrm{~cm}^{-1}$ & This work \\
\hline $\mathrm{C} 45 \mathrm{~A} / \mathrm{K} 54 \mathrm{Q}$ & $E_{452 \mathrm{~nm}}=9530 \mathrm{M}^{-1} \mathrm{~cm}^{-1}$ & This work \\
\hline $\mathrm{C} 45 \mathrm{~S} / \mathrm{K} 54 \mathrm{Q}$ & $\mathrm{E}_{455 \mathrm{~nm}}=9950 \mathrm{M}^{-1} \mathrm{~cm}^{-1}$ & Hopkins \& Williams 1995 \\
\hline $\mathrm{C} 45 \mathrm{~S}$ & $\mathrm{E}_{444 \mathrm{~nm}}=9950 \mathrm{M}^{-1} \mathrm{~cm}^{-1}$ & Hopkins \& Williams 1995 \\
\hline $\mathrm{C} 50 \mathrm{~S}$ & $\mathrm{E}_{444 \mathrm{~nm}}=12140 \mathrm{M}^{-1} \mathrm{~cm}^{-1}$ & Hopkins \& Williams 1995 \\
\hline $\mathrm{C} 45 \mathrm{~S} / \mathrm{C} 50 \mathrm{~S}$ & $\mathrm{E}_{444 \mathrm{~nm}}=12140 \mathrm{M}^{-1} \mathrm{~cm}^{-1}$ & Hopkins \& Williams 1995 \\
\hline $\mathrm{C} 50 \mathrm{M} / \mathrm{K} 54 \mathrm{Q}$ & $\mathrm{E}_{451 \mathrm{~nm}}=10232 \mathrm{M}^{-1} \mathrm{~cm}^{-1}$ & This work \\
\hline C50A & $\mathrm{E}_{455 \mathrm{~nm}}=11300 \mathrm{M}^{-1} \mathrm{~cm}^{-1}$ & This work \\
\hline K54Q & $\mathrm{E}_{455 \mathrm{~nm}}=11300 \mathrm{M}^{-1} \mathrm{~cm}^{-1}$ & Maeda-Yorita et al. 1991 \\
\hline E189Q & $\mathrm{E}_{455 \mathrm{~nm}}=11300 \mathrm{M}^{-1} \mathrm{~cm}^{-1}$ & This work \\
\hline E354K & $\mathrm{E}_{455 \mathrm{~nm}}=11300 \mathrm{M}^{-1} \mathrm{~cm}^{-1}$ & This work \\
\hline R386A & $\mathrm{E}_{455 \mathrm{~nm}}=11300 \mathrm{M}^{-1} \mathrm{~cm}^{-1}$ & This work \\
\hline $\mathrm{H} 445 \mathrm{~A}$ & $\mathrm{E}_{455 \mathrm{~nm}}=11300 \mathrm{M}^{-1} \mathrm{~cm}^{-1}$ & This work \\
\hline E450Q & $\mathrm{E}_{455 \mathrm{~nm}}=11300 \mathrm{M}^{-1} \mathrm{~cm}^{-1}$ & This work \\
\hline
\end{tabular}




\subsubsection{Determination of Redox Potentials of selected $E c E 3$ Variants}

Measurements were performed by Lena-Sophie Dreher in the laboratory of Prof. Bruce Palfey at the University of Michigan, Ann Arbor, USA. The redox potential of FAD bound to $E c E 3$ variants was determined by reductive titration of $\sim 20 \mu \mathrm{M}$ enzyme under anaerobic conditions in presence or absence of $\sim 20 \mu \mathrm{M}$ dye (Table 2.6) (McDonald, Liu, and Palfey 2013). All solutions were made anaerobic by multiple rounds of vacuum and oxygen-free argon flushing. $3 \mathrm{mM}$ sodium dithionite was used to titrate the enzyme, the enzyme-dye mixture and the dye to complete reduction. The titration of the dye in absence of the enzyme served as control. All measurements were carried out at $25{ }^{\circ} \mathrm{C}$ in a $1 \mathrm{~mL}$ reaction volume monitored by a UV-2550 spectrophotometer (Shimadzu Corp.) recording spectra from 300-700 nm. Data were fitted by Nernst analysis of the absorbance changes of enzyme and dye using eq. 2.1 .

$\log \left[\frac{A_{i}-A_{r e d}}{A_{o x}-A_{i}}\right] \mathrm{Enz}=\left(\mathrm{E}_{\mathrm{m} ; \mathrm{dye}}-\mathrm{E}_{\mathrm{m} ; \mathrm{Enz}}\right) \frac{\mathrm{n}_{\mathrm{Enz}} \mathrm{F}}{2.303 \mathrm{RT}}+\frac{\mathrm{n}_{\mathrm{Enz}}}{\mathrm{n}_{\mathrm{dye}}} \log \left[\frac{A_{i}-A_{r e d}}{A_{o x}-A_{i}}\right]$ Dye

Where $\mathrm{n}$ is the number of electrons in the reduction; $\mathrm{F}=$ Faraday's constant; $\mathrm{R}=$ gas constant; $\mathrm{T}=$ absolute temperature; $\mathrm{E}_{\mathrm{m} ; \mathrm{dye}}$ and $\mathrm{E}_{\mathrm{m}, \mathrm{Enz}}$ are the midpoint potentials of the dye and enzyme, respectively; $\mathrm{A}_{\mathrm{i}}$ is an absorbance during the experiment; $\mathrm{A}_{\mathrm{ox}}$ is the absorbance at the start of the experiment when enzyme and dye are oxidized and $\mathrm{A}_{\mathrm{red}}$ is the absorbance at the end of the experiment when enzyme and dye are reduced. Enz and Dye refer to absorbance values sensitive to these components. Corresponding wavelengths of the used enzyme/dye-pairs are listed in Table 2.6. Redox potentials were taken from BIRD and MANSFIELD (Bird and Kuhn 1981; Mansfield 1960). The recorded UV-Vis spectra are listed in the appendix (5.10).

Table 2.6 Redox pairs for the redox potential titrations.

\begin{tabular}{ccc} 
Enzyme-dye pair & wavelengths & $\begin{array}{c}\text { Redox potential (Dye) } \\
\mathbf{E}_{\mathbf{m} 7.6}\end{array}$ \\
\hline EcE3C45A/K54Q / phenosafranin & $403 \mathrm{~nm} / 530 \mathrm{~nm}$ & $-275 \mathrm{mV}$ \\
$E c$ E3C45S/C50S / methylviologen & $450 \mathrm{~nm} / 600 \mathrm{~nm}$ & $-440 \mathrm{mV}$ \\
EcE3C50A / neutral red & $380 \mathrm{~nm} / 550 \mathrm{~nm}$ & $-358 \mathrm{mV}$ \\
\hline
\end{tabular}

2.2.2.10

Photobleaching of EcE3 and Variants thereof

$E c \mathrm{E} 3$ and variants thereof (in $50 \mathrm{mM} \mathrm{KPP} \mathrm{pH} \mathrm{7.6,100} \mathrm{mM} \mathrm{NaCl}$ ) were exposed to $455 \mathrm{~nm}$ light $\left(50 \mathrm{~mW} / \mathrm{cm}^{-2}\right)$ at room temperature and concomitant spectral read-out on a photo diode array attached to an Agilent 8453 UV-visible spectrophotometer. Solution recovery experiments were measured with 
the same experimental setup. EPR samples (2.2.5.4) were taken at time points indicated in the results part and subsequently frozen in liquid nitrogen. Photobleached cofactors were released from the enzyme and analyzed by ESI-TOF-MS (2.2.6.5).

\subsubsection{Substrates and Substrate Analogs}

Substrates used for functional assays and for crystallization were purchased from Sigma-Aldrich or AppliChem.

\subsubsection{Concentration Determination of Substrates and Cofactors}

The concentration of substrates and the cofactor analogues was determined spectroscopically using molecular absorbance coefficients listed in Table 2.7.

Table 2.7 Spectroscopic characteristics and structures of relevant cofactors.

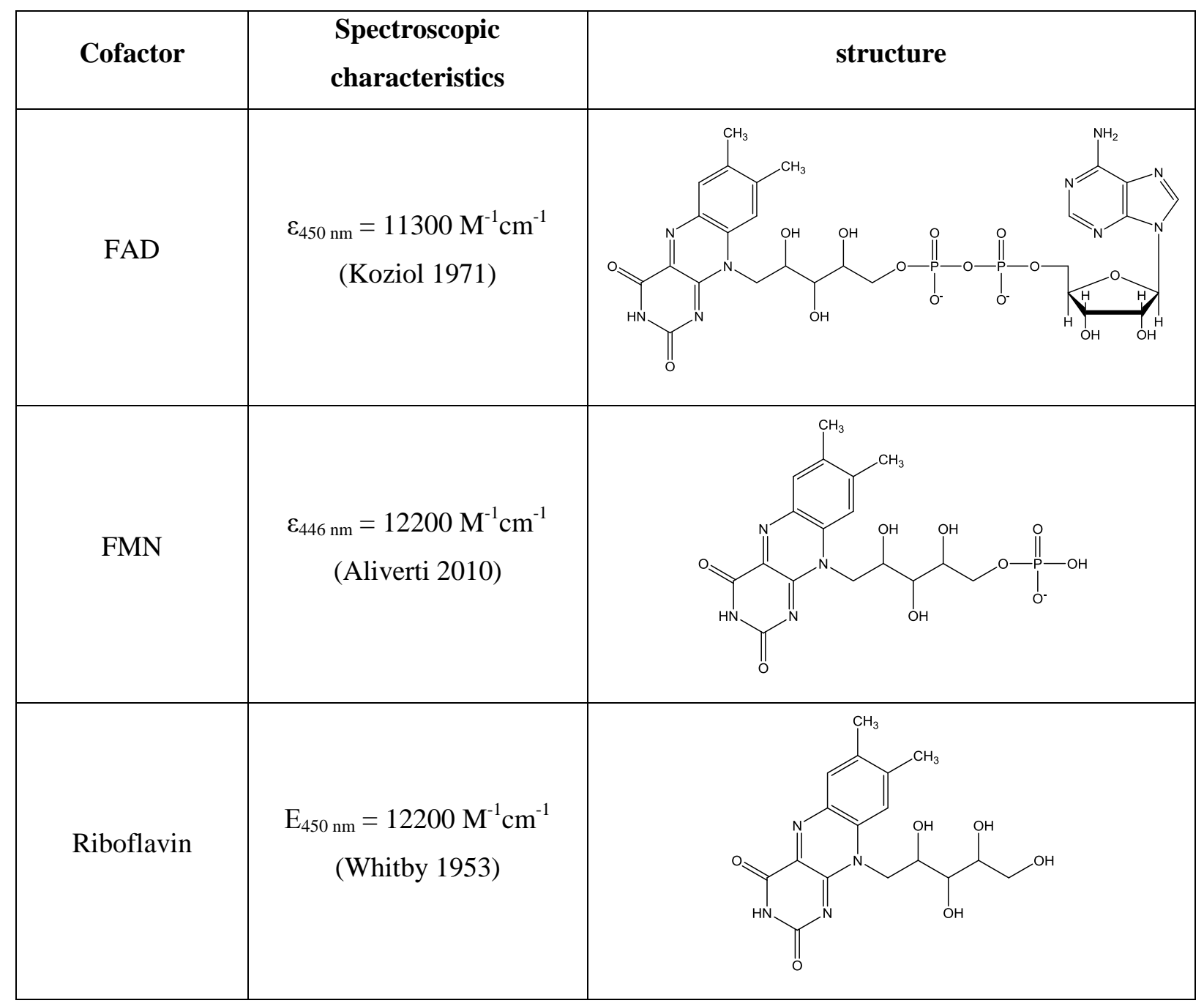




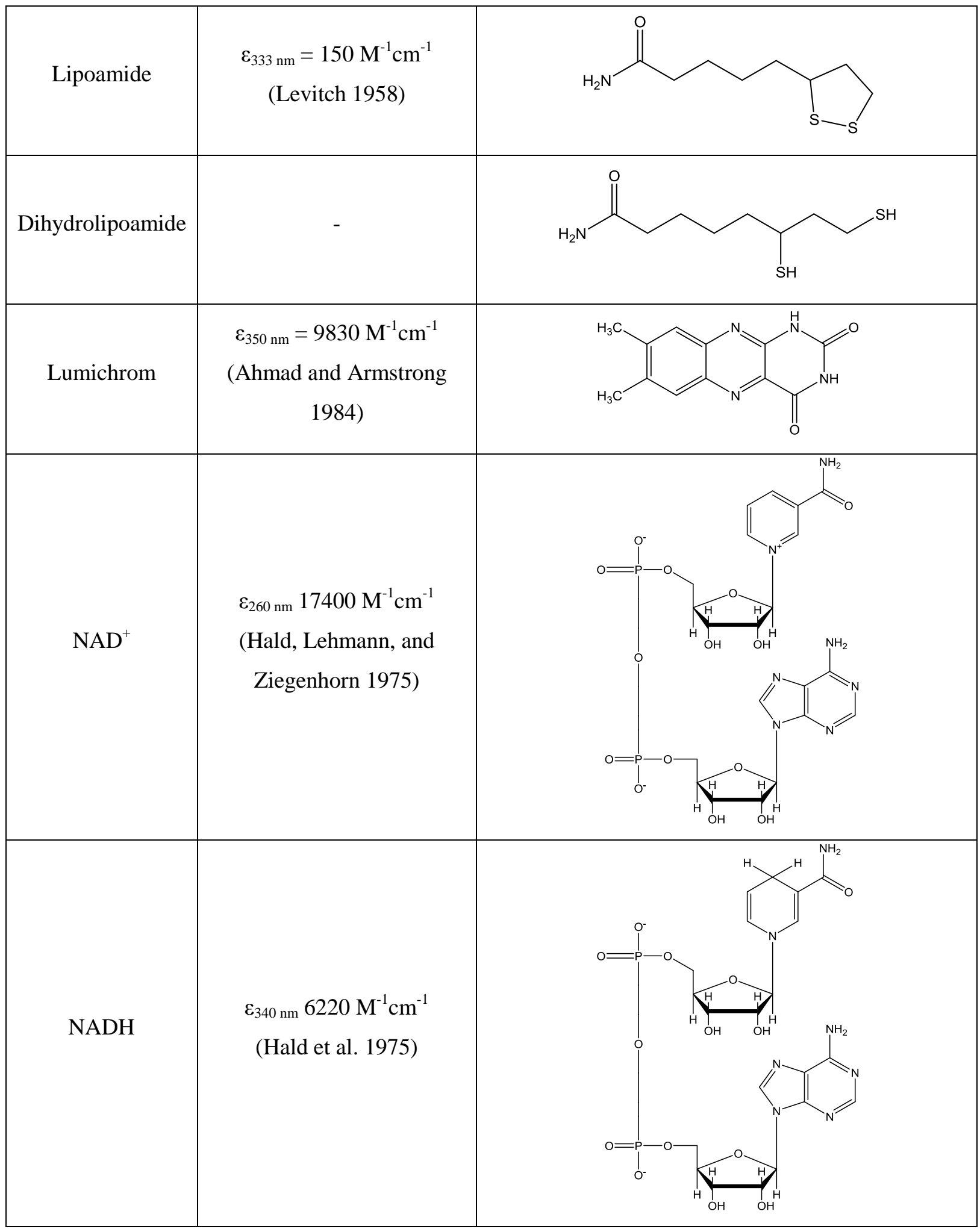




\subsubsection{Kinetic Methods}

\subsubsection{Steady-State Activity Assay}

The activity of $E c \mathrm{E} 3$ and all variants was determined under pseudo steady state conditions in the presence of $100 \mu \mathrm{M}$ NADH, $500 \mu \mathrm{M} \mathrm{NAD}^{+}, 0.125-3 \mathrm{mM}$ lipoamide and $5.25 \%$ ethanol in $50 \mathrm{mM}$ $\mathrm{KPP}$ pH 7.6 and $100 \mathrm{mM} \mathrm{NaCl}$. Measurements were carried out at $20^{\circ} \mathrm{C}$ and the reaction was started by addition of the enzyme while the consumption of NADH was tracked at $340 \mathrm{~nm}$. If applicable, the dependency of the initial rates on the substrate concentration was analyzed according to

eq. 2.2. It should be noted that the solubility of lipoamide is limited at $3 \mathrm{mM}$ in the used buffer condition resulting in an underdetermined. Therefore, the determined specific activities represent the maximum substrate turnover in the presence of $3 \mathrm{mM}$ lipoamide and $500 \mu \mathrm{M} \mathrm{NAD}^{+}$at $\mathrm{pH}$ 7.6.

$$
\mathrm{V}=\frac{\mathrm{V}_{\max } \cdot[\mathrm{S}]}{K_{\mathrm{M}}+[\mathrm{S}]}
$$

\subsubsection{2 $\mathrm{NAD}^{+}$activation Experiments}

$\mathrm{NAD}^{+}$activation experiments were carried out under the same conditions as described for the steadystate activity assay. The lipoamide was kept constant at a concentration of $3 \mathrm{mM}$ while the $\mathrm{NAD}^{+}$ concentration was varied between 0 and $3 \mathrm{mM}$. The dependency of the initial rates on the $\mathrm{NAD}^{+}$ concentration was used to determine the specific activity.

\subsubsection{3 pH Dependency Experiments under a constant ionic Strength}

The $\mathrm{pH}$ dependency of the $E c \mathrm{E} 3$ activity was determined under steady state conditions (2.2.4.1) in the presence of $100 \mu \mathrm{M} \mathrm{NADH}, 0-500 \mu \mathrm{M} \mathrm{NAD}{ }^{+}, 3 \mathrm{mM}$ lipoamide and $5.25 \%$ ethanol. A mixture comprised of $50 \mathrm{mM}$ Acetic Acid, $50 \mathrm{mM}$ MES and $100 \mathrm{mM}$ Tris served as buffer system ensuring a constant ionic strength in a $\mathrm{pH}$ range from 4.5 to 9.5 (Ellis and Morrison 1982). Measurements were carried out at $20^{\circ} \mathrm{C}$ and the reaction was started by addition of the enzyme while the consumption of $\mathrm{NADH}$ was tracked at $340 \mathrm{~nm}$. The dependency of the initial rates on the $\mathrm{pH}$ was used to determine the $\mathrm{pH}$ optimum.

\subsubsection{Fast Kinetics-Stopped Flow Absorbance Spectroscopy}

Rapid reaction measurements experiments were carried out on an Applied Photophysics SX20 MV stopped-flow spectrophotometer at $4{ }^{\circ} \mathrm{C}$. Spectra were recorded in equidistant time intervals on a photo diode array detector whereas single wavelength measurements were recorded with a logarithmic 
time scale. Final concentrations of enzymes, cofactors and substrates after mixing are shown individually in the results part.

Single mixing experiments

Single wavelength measurements were performed at wavelengths of $380,487,523$ and $650 \mathrm{~nm}$. Enzyme and substrates were mixed in all cases in a 1 to 1 volume ratio. The final concentrations of enzymes, cofactors and substrates after mixing are shown in the figures in the results part.

\section{Sequential mixing experiments}

Sequential mixing experiments were used to study the reoxidation of the two-electron reduced state $\left(\mathrm{EH}_{2}\right)$ of $E c \mathrm{E} 3$ by $\mathrm{NAD}^{+}$. First, $E c \mathrm{E} 3-\mathrm{EH}_{2}$ was formed by pre-mixing $E c \mathrm{E} 3$ and $E c \mathrm{Lip} 3 \mathrm{H}_{2}$ (both at the same concentrations) followed by mixing with the final electron acceptor $\mathrm{NAD}^{+}$. The optimal time point for the second mixing step was obtained from the single mixing experiments. The final concentrations of enzymes, cofactors and substrates after the second mixing step are shown in the figures in the results part.

\subsubsection{Biophysical Methods}

\subsubsection{UV-Vis Spectroscopy}

UV-Vis spectra were collected between $300-700 \mathrm{~nm}$ in a Jasco V-650 spectrophotometer using Quartz cuvettes with a path length of $1 \mathrm{~cm}$ and a band width of $1 \mathrm{~nm}$. The acquisition speed was $100 \mathrm{~nm} / \mathrm{min}$ with a data pitch of $1 \mathrm{~nm}$. As buffer system for all UV-Vis experiments served $50 \mathrm{mM}$ potassium phosphate $\mathrm{pH} 7.6$ and $100 \mathrm{mM} \mathrm{NaCl}$.

\subsubsection{CD spectroscopy}

Circular dichroism spectra were recorded for $E c \mathrm{E} 3$ and $E c L i p 3$ including active site variants with a Chirascan CD-spectropolarimeter. Secondary structure elements were detected by collection of far UV spectra between $180-260 \mathrm{~nm}$ with a path length of $1 \mathrm{~mm}$ at in $50 \mathrm{mM}$ potassium phosphate buffer $\mathrm{pH}$ 7.6. Near UV/Vis spectra were collected between $300-500 \mathrm{~nm}$ with a path length of $1 \mathrm{~cm}$. Used buffers and added substrates are indicated in the figure legend. 
Table 2.8 Acquisition parameters for CD spectroscopy experiments

\begin{tabular}{ccc} 
& Far UV spectra & Near UV spectra \\
\hline enzyme concentration & $0.1-0.2 \mathrm{mg} / \mathrm{mL}$ & $2.5 \mathrm{mg} / \mathrm{mL}$ \\
data interval & $1 \mathrm{~nm}$ & $1 \mathrm{~nm}$ \\
band width & $1 \mathrm{~nm}$ & $1 \mathrm{~nm}$ \\
response & $2 \mathrm{~s}$ & $3 \mathrm{~s}$ \\
path length & $1 \mathrm{~mm}$ & $1 \mathrm{~cm}$ \\
wavelength & $180-260 \mathrm{~nm}$ & $300-500 \mathrm{~nm}$ \\
\hline
\end{tabular}

\subsubsection{Isothermal Titration Calorimetry}

Isothermal titration calorimetry (ITC) allows the direct and quantitative determination of the binding thermodynamics of a biological process. Under optimal conditions the association constant, the binding enthalpy, the binding entropy and the stoichiometry of a binding event can be calculated from a single ITC experiment. An ITC200 MicroCalorimeter system was used to investigate both the interaction between $E c \mathrm{E} 3$ and $E c \mathrm{Lip} 3$ and the cofactor binding of $\mathrm{NAD}^{+}$to $E c \mathrm{E} 3$ and variants thereof.

The enzymes were buffer exchanged into the same buffer (50 mM KPP pH 7.6, $100 \mathrm{mM} \mathrm{NaCl}$ ). The ITC cell was filled with $100 \mu \mathrm{M}$ (active sites) $E c$ E3. The injection syringe was filled with $1 \mathrm{mM}$ of $E c$ Lip3. Measurements were performed at $20{ }^{\circ} \mathrm{C}$ with a stirring speed of $500 \mathrm{~min}^{-1}$. A titration of $1 \mathrm{mM}$ EcLip3 into buffer served as control to check for heat effects due to dilution. The interaction of $\mathrm{NAD}^{+}$with the enzymes was assayed under the same conditions while dissolving $\mathrm{NAD}^{+}$directly in the buffer system.

\subsubsection{EPR}

Measurements on EcE3C45A/K54Q (pseudoLOV) were performed by Christopher Engelhard in the laboratory of Prof. Robert Bittl at the Freie Universität Berlin. Acquisition parameters were as follows:

-Modulation amplitude: 3G

- Time constant: 30ms

- Lock-in frequency $100 \mathrm{kHz}$

- Sensitivity: $1 \mathrm{mV}$

- Microwave frequency: 9.38 GHz, all spectra frequency-corrected to $9.60 \mathrm{GHz}$

- Microwave power: $63.2 \mathrm{nW}(-35 \mathrm{~dB}$ on a standard $200 \mathrm{~mW}$ microwave source) 


\subsubsection{Mass Spectrometry}

\subsubsection{MALDI-TOF Mass Spectrometry}

It could be shown that MALDI-TOF Mass Spectrometry is suitable method to detect the state of a lipoylation of an isolated hybrid lipoyl domain of E. coli PDHc (Wei et al. 2003). This method was also applied to the lipoyl domain II of the human PDHc by members of this group (Güttler 2008; Seifert 2010). In both cases, the entire domain was subjected to MALDI-TOF MS. In this work a MALDI-TOF mass spectrometric approach of an isolated lipoyl domain of the E. coli PDH after tryptic digestion of the latter will be presented.

\subsubsection{Determination of the Lipoylation State of $E c$ Lip3}

Digestion of a protein with trypsin leads to fragments showing specific cleavages after arginine or lysine residues, respectively. These tryptic peptides are then used for exact mass determination, also allowing the detection of putative post-translational modifications of amino acid side chains. On the one hand, the lipoamide cofactor bound to EcLip3 directly leads to an increase in mass of the distinct peptide on the other hand it also leads to an altered digestion pattern since the covalent bond between lipoic acid and the $\varepsilon$-amino group of lysine 45 prevents a tryptic digestion at this site. Tryptic peptides of $E c$ Lip3 including their corresponding masses were predicted with the PeptideCutter interface (Table 5.1). The peptides required for analysis of the lipoylation state of $E c L i p 3$ are listed in Table 2.9.

Table 2.9 Possible tryptic fragments of EcLip3 for the lipoylation state analysis

\begin{tabular}{ccc} 
Peptide sequence & $\begin{array}{c}\text { Peptide mass } \\
{[\text { Da] }}\end{array}$ & property \\
\hline VAAEQSLITVEGDK* & 1459.6 & $\begin{array}{c}\text { unlipoylated I } \\
\text { unlipoylated II } \\
\text { ASMEVPAPFAGVVK* }\end{array}$ \\
lipoylated \\
\hline VAAEQSLITVEGDK(lip)ASMEVPAPFAGVVK & 3032.5 & \\
\hline
\end{tabular}

In the case of fully lipoylated EcLip3, the peptides unlipoylated I and II are not expected occur since trypsin will not cleave due to the covalent attachment of lipoic acid to lysine45 (highlighted in yellow). In contrast, only one bigger fragment should appear after tryptic digestion (lipoylated). This fragment has a total mass of $3032.5 \mathrm{Da}$ comprising the masses of the two individual fragments (unlipoylated $I$ and $I I$ ) including the bound lipoamide cofactor. 


\subsubsection{MALDI-TOF Sample Preparation}

Samples of $E c L i p 3$ for tryptic digestion were taken after purification. $15 \mu \mathrm{L}$ sample were added to $25 \mu \mathrm{L} 25 \mathrm{mM}$ ammonium hydrogen carbonate and $10 \mu \mathrm{L}$ trypsin $(100 \mu \mathrm{g} / \mathrm{mL})$. After an overnight digestion at $37{ }^{\circ} \mathrm{C}$ the reaction was stopped by the addition of $85 \%$ FA in a 1:1 volume ratio. MALDITOF samples were desalted using ZipTips pipette tips containing $\mathrm{C}_{18}$-silica beads with a binding capacity of $5 \mu \mathrm{g}$ protein. ZipTips were washed three times with a solution made of $10 \mu \mathrm{L} 70 \% \mathrm{ACN} /$ $0.3 \%$ FA followed by an equilibration with $10 \mu \mathrm{L} 1 \%$ FA. After the loading of the sample the column was washed again with $10 \mu \mathrm{L} 1 \% \mathrm{FA}$ as described above. Tryptic peptides were eluted with $10 \mu \mathrm{L}$ of $70 \%$ ACN / $0.3 \%$ FA Table 2.10 .

Table 2.10 ZipTip preparation for MALDI-TOF samples

\begin{tabular}{ccc} 
Desalting step & Cycles & Solution \\
\hline Washing & $3 \mathrm{x}$ & $70 \% \mathrm{ACN} / 0.3 \% \mathrm{FA}$ \\
Equilibration & $5 \mathrm{x}$ & $1 \% \mathrm{FA}$ \\
Loading & $5 \mathrm{x}$ & Sample \\
Washing & $5 \mathrm{x}$ & $1 \% \mathrm{FA}$ \\
Elution & $1 \mathrm{x}$ & $70 \% \mathrm{ACN} / 0.3 \% \mathrm{FA}$ \\
\hline
\end{tabular}

\subsubsection{MALDI-TOF Measurement and Data Evaluation}

The MALDI-TOF device was calibrated using the Peptide Calibration Standard (Table 2.11 and Figure 2.3). $2 \mu \mathrm{L}$ of desalted sample were mixed with $2 \mu \mathrm{L}$ of saturated DHB matrix solution. $2 \mu \mathrm{L}$ thereof were then pipetted on a MTP 384 polished steel sample carrier. The mixture was dried on air. Data were acquired in a mass range from $\mathrm{m} / \mathrm{z} 650$ to 3500 using an acceleration voltage of $20 \mathrm{kV} .40$ shots were collected with a laser intensity of $40 \%$ per measurement. In total, ten measurements were carried out resulting in the accumulation of 400 shots. Data acquisition and analysis were done using the Flex analysis software package and corresponding graphs were compiled with SigmaPlot. 
Table 2.11 Expected and acquired masses of the Peptide Calibration Standard

\begin{tabular}{ccc}
$\begin{array}{c}\text { Peptide Calibration } \\
\text { Standard }\end{array}$ & $\begin{array}{c}{[\mathbf{M + H}]^{+}} \\
\text {Mono isotopic } \\
\text { expected }\end{array}$ & $\begin{array}{c}{[\mathbf{M + H}]^{+}} \\
\text {Mono isotopic } \\
\text { acquired }\end{array}$ \\
\hline Angiotensin II & 1046.51 & 1046.18 \\
Angiotensin I & 1296.68 & 1296.25 \\
Substance P & 1347.73 & 1347.27 \\
Bombesin & 1619.82 & 1619.25 \\
ACTH clip 1-17 & 2093.08 & 2093.96 \\
ACTH clip 18-39 & 2465.19 & 2465.33 \\
\hline
\end{tabular}

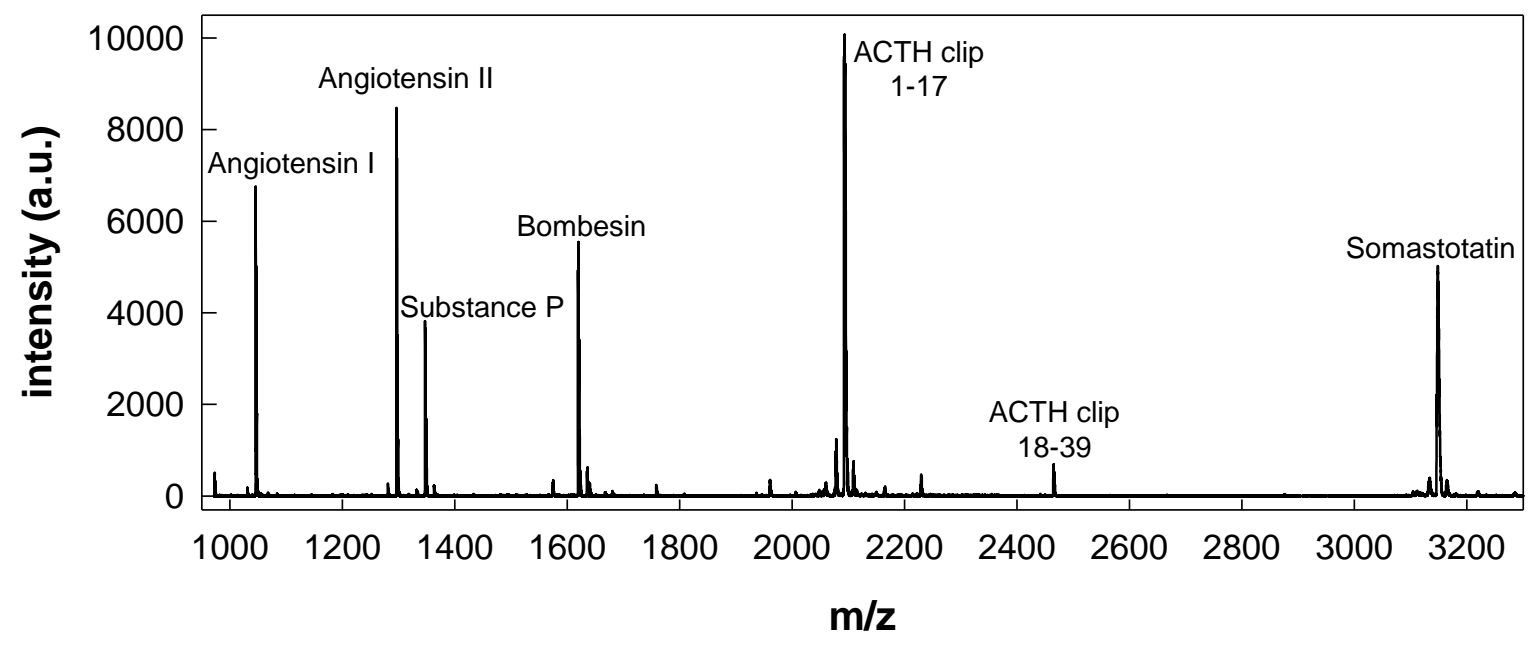

Figure 2.3 MALDI-TOF mass spectrum of the Peptide Calibration Standard

\subsubsection{UPLC-ESI-TOF Sample Preparation}

The enzyme-bound flavin cofactor was released before and after photoexcitation (2.2.2.10), respectively, by different protein precipitation methods. First, $E c \mathrm{E} 3$ and variants thereof were heated to $95^{\circ} \mathrm{C}$ for ten minutes with occasional vortexing. Secondly, $E c \mathrm{E} 3$ was precipitated by addition of ice cold $50 \%(\mathrm{w} / \mathrm{v})$ TCA to a final concentration of $5 \%(\mathrm{w} / \mathrm{v})$ followed by an incubation on ice for ten minutes. Thirdly, one volume of protein solution was mixed with four volumes of ice cold acetone followed by an incubation step at $-70{ }^{\circ} \mathrm{C}$ for ten minutes. In all cases precipitated protein was removed by three rounds of centrifugation at $21,000 \mathrm{x}$ g for 30 minutes at $4{ }^{\circ} \mathrm{C}$. Prior to MS and MS/MS analyses, the resulting supernatant containing the released cofactor was diluted with $\mathrm{ddH}_{2} \mathrm{O}$ as indicated in the results part. 


\subsubsection{UPLC-ESI-TOF-MS}

Analysis of the released $E c E 3$ cofactor was performed on an ultra-performance liquid chromatography (UPLC) ACQUITY system coupled to an UPLC eLambda $800 \mathrm{~nm}$ photo diode array detector (Waters Corporation, Milford, USA), followed by an LCT Premier orthogonal electrospray ionization time-offlight mass spectrometer (ESI-TOF-MS, Waters Corporation, Milford, USA). For analysis, the UPLC was equipped with an ACQUITY UPLC HSS T3 column $(1.0 \times 100 \mathrm{~mm}, 1.8 \mu \mathrm{m}$ particle size, Waters Corporation, Milford, USA) held at $40{ }^{\circ} \mathrm{C}$ and a flow rate of $0.2 \mathrm{~mL} / \mathrm{min}$. The following gradient program was run: $0-0.5 \min 1 \% \mathrm{~B}, 0.5-3 \min 1 \% \mathrm{~B}$ to $20 \% \mathrm{~B}, 3-8 \min 20 \% \mathrm{~B}$ to $100 \% \mathrm{~B}, 8-$ $10 \min 100 \% \mathrm{~B}, 10-10.1 \min 100 \% \mathrm{~B}$ to $1 \% \mathrm{~B}, 10.1-14 \min 1 \% \mathrm{~B}$ (solvent system $\mathrm{A}$ : water:formic acid (100:0.1, v/v); B: acetonitrile:formic acid (100:0.1, v/v)). UV-VIS data were collected between $190-800 \mathrm{~nm}$ in $1.2 \mathrm{~nm}$ steps.

The ESI-TOF-MS was run at negative ionization mode for all samples. Data were collected over a mass range from $\mathrm{m} / \mathrm{z} 50.00-1200.00$ and a runtime from $0-13$ min using a capillary voltage of $2500 \mathrm{~V}$. The cone voltage was maintained at $30 \mathrm{~V}$, the desolvation temperature at $350{ }^{\circ} \mathrm{C}$ and the source temperature at $80^{\circ} \mathrm{C}$. Data acquisition was carried out by using the MassLynx software in centroid data format. Nitrogen was used as cone and desolvation gas at a flow of 30 and $800 \mathrm{l} / \mathrm{h}$, respectively. The dynamic range enhancement mode was used for data recording. All analyses were calibrated by applying the lock spray reference compound leucine-enkephaline ([M-H] 554.262 as well as its $13 \mathrm{C}$ isotopologue ([M-H $]^{-}$555.2653; Sigma-Aldrich, Deisenheim, Germany) at a concentration of $0.5 \mu \mathrm{g} / \mathrm{mL}$ in acetonitrile:water (50:50, v/v) and a flow rate of $20 \mu \mathrm{L} / \mathrm{min}$. The measurements for the lumichrom reference were run at positive ionization mode using the same parameters except a capillary voltage of $2700 \mathrm{~V}$ and a calibration by applying the aforementioned lock spray reference $\left([\mathrm{M}+\mathrm{H}]^{+} 556.2766\right.$ as well as its $2 \times 13 \mathrm{C}$ isotopologue $\left.[\mathrm{M}+\mathrm{H}]^{+} 558.2833\right)$. UPLC-ESITOF-MS measurements and subsequent data analysis were carried out by Dr. Kirstin Feussner.

\subsubsection{UHPLC-ESI-QTOF-MS}

MS/MS fragmentation experiments were performed via UHPLC-ESI-QTOF-MS. For UHPLC, an Agilent 1290 Infinity series UHPLC system (Agilent Technologies, Böblingen, Germany) with an ACQUITY UPLC BEH C18 column $(2.1$ x $100 \mathrm{~mm}, 1.7 \mu \mathrm{m}$ particle size, Waters Corporation, Milford, USA) kept at $40{ }^{\circ} \mathrm{C}$ was used with a flow rate of $0.5 \mathrm{~mL} / \mathrm{min}$. The solvent system and the applied gradient were comparable to that of method 2.2.6.6. Mass detection was performed with an Agilent 6540 UH Accurate-Mass-Q-TOF MS (Agilent Technologies, Böblingen, Germany). The MS was operated using an ESI source with Agilent Dual jet Stream Technology (Agilent Technologies, Böblingen, Germany) in negative and positive ionization mode. Ionization parameters were as follows: gas temperature $300{ }^{\circ} \mathrm{C}$, gas flow $8 \mathrm{~L} / \mathrm{min}$, nebulizer pressure $35 \mathrm{psi}$, sheath gas temperature $350{ }^{\circ} \mathrm{C}$, 
sheath gas flow $11 \mathrm{~L} / \mathrm{min}$, VCap $3 \mathrm{kV}$, nozzle voltage $100 \mathrm{~V}$. As a collision cell, a linear hexapole collision cell with nitrogen as collision gas was used with collision energies of $40 \mathrm{eV}-45 \mathrm{eV}$. For data acquisition, the Mass Hunter Workstation Acquisition Software was used (B.05.01.), with Mass Hunter Qualitative Analysis software (B.06.00) as analysis tool. For structure elucidation the MS/MS spectra of FAD derivatives were interpreted in comparison to FAD spectra (METLIN ID2302) deposited in the METLIN database. UHPLC-ESI-QTOF-MS measurements and subsequent data analysis were carried out by Dr. Kirstin Feussner

\subsubsection{X-ray Crystallography}

X-ray diffraction datasets, collected in-house or at different synchrotron radiation facilities (BESSYII, Berlin; DESY, Hamburg), were processed with XDS (Kabsch 2010). Protein models were iteratively refined and improved with PHENIX (Adams et al. 2010) and validated with MOLPROBITY (Davis et al. 2007).

\subsubsection{Crystallization}

\section{Crystallization of $E c \mathrm{E} 3$}

Crystals were obtained using the hanging drop vapor-diffusion method. $E c$ E3 (derived from pGS523) was concentrated to $14-16 \mathrm{mg} / \mathrm{mL}$ in $50 \mathrm{mM}$ imidazole $\mathrm{pH} 6.5,150 \mathrm{mM} \mathrm{NaCl}$. All further steps were performed at 6-8 ${ }^{\circ} \mathrm{C}$. EcE3 was mixed with equal amounts of reservoir solution, comprising 1.8 2.4 M ammonium sulfate and 0.1 M potassium phosphate buffer $\mathrm{pH}$ 7.0. Drop size was $4 \mu \mathrm{L}$. Crystals were cryoprotected using reservoir solution supplemented with $25 \%$ glycerol (v/v) before flash freezing and storage in liquid nitrogen. For substrate soaking experiments the cryo solution was supplemented with $5 \mathrm{mM} \mathrm{NAD}{ }^{+}$.

\section{Crystallization of $E c \mathrm{E} 3 \mathrm{C} 45 \mathrm{~S}$}

EcE3C45S was desalted against $50 \mathrm{mM}$ imidazole $\mathrm{pH} 6.5,150 \mathrm{mM} \mathrm{NaCl}$ and concentrated to $13.5 \mathrm{mg} / \mathrm{mL}$. The protein solution was mixed with equal amounts of reservoir solution, comprising 2.4 M ammonium sulfate and 0.1 M MES buffer $\mathrm{pH}$ 6.5. Directly after preparation drops were microseeded with wild type crystals pre-grown in $8-13 \%$ (w/v) PEG 4000, $200 \mathrm{mM} \mathrm{NaCl}, 1-4 \%$ (v/v) PEG400 and 0.1 M MES pH 6.5. Crystals were cryoprotected the same way as the crystals of the wild type enzyme. 


\subsubsection{Data Processing and Molecular Replacement}

Datasets were processed and scaled with XDS (Kabsch 2010). Data processing was mainly done by Dr. Piotr Neumann (Department for Molecular Structural Biology, University of Göttingen). Data collection- and structure refinement statistics can be found in the appendix (Table 5.3). The structure of $E c \mathrm{E} 3$ was determined by molecular replacement using dihydrolipoamide dehydrogenase of Neisseria meningitidis (PDB: 1OJT) as initial search model with PHASER (McCoy 2006).

\subsubsection{Model Building, Refinement and Validation}

Structure refinement was carried out using PHENIX (Adams et al. 2010) employing the maximum likelihood method, with 1.5 to $5 \%$ of randomly chosen data for validation by the $\mathrm{R}_{\text {free }}$ factor (Brünger 1992). After a rigid body refinement models were inspected manually with COOT (Emsley et al. 2010) checking the conformation of side chains and the position of water molecules or other ligands. Validation of the refined models was carried out using COOT and MOLPROBITY. Sigma A-weighted electron density maps $(2 \mathrm{mFo}-\mathrm{DFc}, \mathrm{mFo}-\mathrm{DFc})$ were generated with PHENIX. Simulated-annealing (SA) omits maps were calculated as implemented in CNS (Brunger 2007) by Dr. Piotr Neumann (Department for Molecular Structural Biology, University of Göttingen). Figures were compiled with PYMOL (DeLano 2003). 


\section{Results and Discussion}

\subsection{Spectroscopic and Crystallographic Characterization of EcE3}

Major emphasis was devoted to the spectroscopic and crystallographic detection of reaction intermediates of the $E c \mathrm{E} 3$ reaction cycle. Therefore a convenient and robust expression system has been established which allowed the subsequent study of the wild type enzyme and selected active site variants thereof. These enzymes were first characterized by conventional UV-Vis spectroscopy and steady-state kinetics and then further investigated by stopped-flow absorbance spectroscopy and transient kinetic analyses. Based on these results crystallization trials with active site mutants capable of stabilizing a distinct reaction step were conducted to expand the gained spectroscopic knowledge by crystallographic data.

\subsubsection{Kinetic Characterization of EcE3 in dependence of Lipoamide, $\mathrm{NAD}^{+}$and $\mathrm{pH}$}

Steady-state parameters of lipoamide dehydrogenase from Escherichia coli have been investigated in detail over the last decades (Wilkinson and Williams 1981; Williams 1965; Williams et al. 1967). In addition, the mechanism of the severe product inhibition by NADH could be determined in significant detail (Wilkinson and Williams 1981). The following results briefly summarize the effects of lipoamide, $\mathrm{NAD}^{+}$and $\mathrm{pH}$ on the steady-state catalysis of $E c \mathrm{E} 3$ purified from modern expression systems and demonstrate the consistency with already published data (Figure 3.1). In the absence of $\mathrm{NAD}^{+}$the initial velocity of $E c \mathrm{E} 3$ is very low when tracking the reverse reaction with $\mathrm{NADH}$ as reductant and lipoamide as electron acceptor. With increasing $\mathrm{NAD}^{+}$concentrations the inhibitory effect of NADH is overcome leading to enhanced initial rates (Figure 3.1A). The activation by NAD ${ }^{+}$ is complete at about $2.0 \mathrm{mM}$ added $\mathrm{NAD}^{+}$under the conditions tested (Figure 3.1B). The apparent binding constant was determined to be $400 \mu \mathrm{M}$ (Wilkinson and Williams 1981). Although the activating effect of $\mathrm{NAD}^{+}$is unquestionable the absolute influence of the pyridine nucleotide is also pH dependent (Figure 3.1C) (Reed, Koike, and Shah 1960; Wilkinson and Williams 1981). At all tested $\mathrm{pH}$ values the reverse reaction is very slow without externally added $\mathrm{NAD}^{+}$. In the presence of $50 \mu \mathrm{M} \mathrm{NAD}{ }^{+}$the enzyme shows a clear catalytic optimum at $\mathrm{pH} 8.5$ harboring a $\sim 5$-fold increased reaction rate compared to the reactions lacking externally added $\mathrm{NAD}^{+}$. When adding $500 \mu \mathrm{M} \mathrm{NAD}^{+}$ the $\mathrm{pH}$ profile is bell shaped peaking at $\mathrm{pH}$ 8.0. It is obvious that the chosen $\mathrm{pH}$ acts as a determinate whether $\mathrm{NAD}^{+}$functions only as an activator or, at higher concentrations, both at activator and an inhibitor. This phenomenon has already been discussed elsewhere (Wilkinson and Williams 1981). 
A standard steady-state assay was therefore used to compare the relative activities of active site mutants during the course of the study. This assay comprised $500 \mu \mathrm{M} \mathrm{NAD}^{+}, 3 \mathrm{mM}$ lipoamide and $100 \mu \mathrm{M} N A D H$ at $\mathrm{pH}$ 7.6. The chosen $\mathrm{pH}$ was a consequence of high catalytic activity with respect to $E c \mathrm{E} 3$ but was further determined by the usage of an isolated lipoyl domain of the $E c \mathrm{E} 2$ component (EcLip3) as reductant to track the reductive half-reaction (3.2). This isolated domain showed to be most stable at $\mathrm{pH} 7.6$ and thus a pH below 8.0 was obligate for further investigations. Hence, the used standard steady-state assay reflected a compromise with respect to activity of $E c \mathrm{E} 3$ and stability of $E c$ Lip3 at $\mathrm{pH}$ 7.6. The resulting steady-state kinetic under the assay conditions chosen for $E c \mathrm{E} 3$ is shown in Figure 3.1D. Due to the limited solubility of lipoamide in aqueous solutions (Argyrou and Blanchard 2001) the resulting plot lacks a hyperbolic shape. Catalytic activities will therefore be referred to units/mg. Catalytic activities for all investigated (double) variants of $E c E 3$ under the conditions described can be found in the appendix (5.4). 

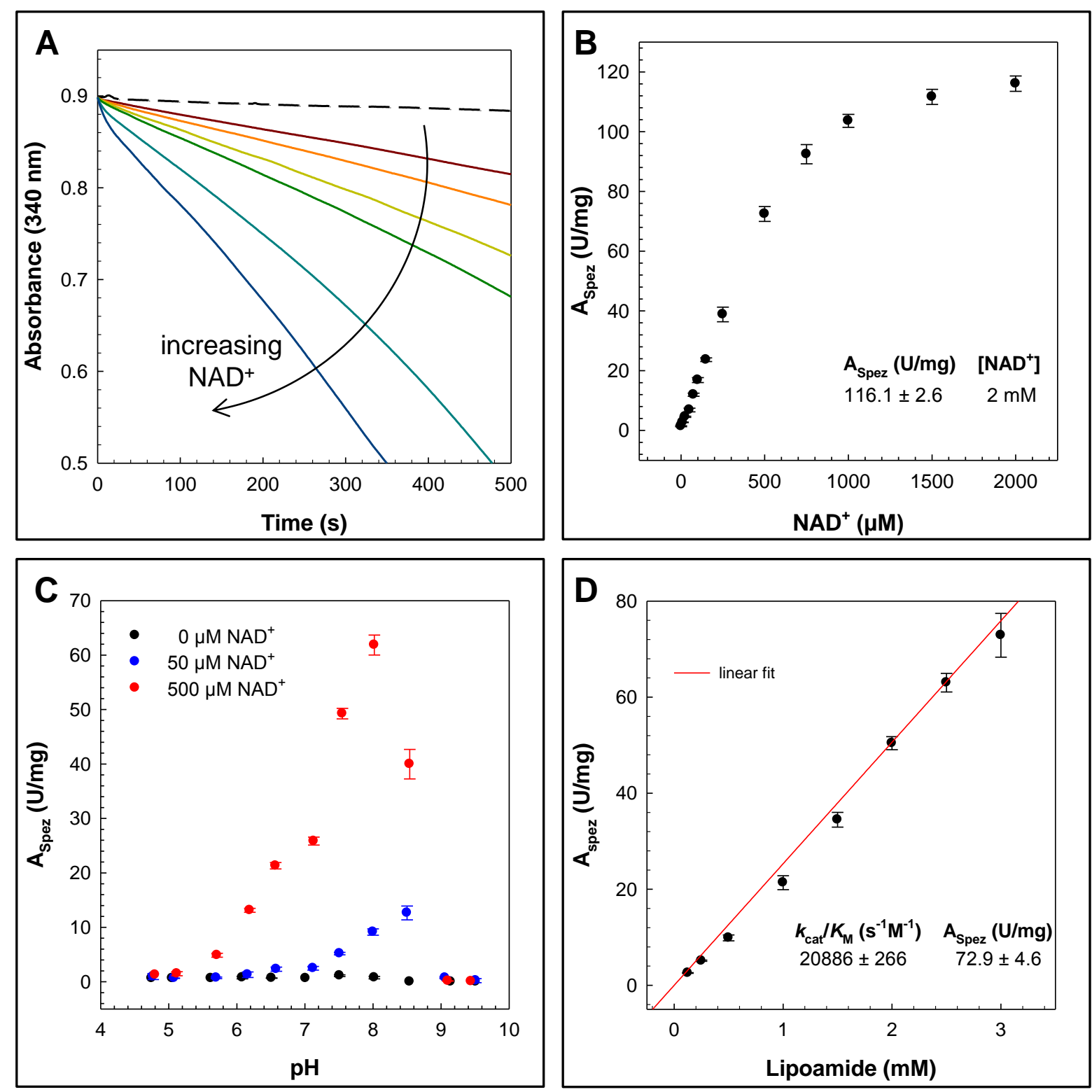

Figure 3.1 Steady-state characterization of $E c E 3$.

(A) Time course of the NADH-lipoamide reaction, effect of added $\mathrm{NAD}^{+}$. The assay comprised $100 \mu \mathrm{M}$ NADH and $3 \mathrm{mM}$ lipoamide. (B) Effect of $\mathrm{NAD}^{+}$on the observed initial rates in the $\mathrm{NADH}-$ lipoamide reaction. Conditions of the assay are as in $\mathrm{A}$, except that $\mathrm{NAD}^{+}$was added at the indicated concentrations before the addition of enzyme. (C) $\mathrm{pH}$ activity profile for the NADHlipoamide reaction, effect of added NAD. (D) Lipoamide dependence standard assay of EcE3 comprising $500 \mu \mathrm{M} \mathrm{NAD}^{+} 3 \mathrm{mM}$ lipoamide and $100 \mu \mathrm{M}$ NADH. The reaction was started by addition of the enzyme. All experiments were carried out at $\mathrm{pH}$ 7.6. 


\subsubsection{Detection of Reaction Intermediates using Stopped-flow Absorbance Spectroscopy}

Besides the establishment of a standard steady-state assay also transient kinetic analyses should be performed at appropriate conditions using stopped-flow absorbance spectroscopy. It was demonstrated that this technique is suitable to study both the spectroscopic and kinetic properties of lipoamide dehydrogenase from Mycobacterium tuberculosis (Argyrou et al. 2002). Hence, the kinetic rate constants for single reaction steps could be determined in significant detail. It was therefore the aim to transfer the method established by ARGYROU and co-workers to the E. coli system which consequently allowed the spectroscopic characterization of the $E c \mathrm{E} 3 \mathrm{wt}$ and active site mutants. Major emphasis was thereby devoted to the detection of the covalent FAD-C4a-cysteinyl adduct.

As described for all lipoamide dehydrogenases the reaction cycle consist of multiple reaction steps, which are completely reversible (Massey, Gibson, and Veeger 1960). This allows to follow the reaction cycle from both half-reactions, from the reductive half-reaction (dihydrolipoamide as reductant; forward direction) or from the oxidative half-reaction (NADH as reductant; reverse direction). Due to the absence of commercially available dihydrolipoamide the reduced pyridine nucleotide was chosen as reductant, which harbors further advantages compared to dihydrolipoamide besides its unlimited availability. In contrast to lipoamide, NADH does not show limited solubility in aqueous solution as (dihydro)lipoamide does (Argyrou and Blanchard 2001) and secondly, it was demonstrated that NADH has the ability to overreduce lipoamide dehydrogenases to the $\mathrm{EH}_{4}$ state, which allows the observation of further reaction intermediates (Argyrou et al. 2003, 2002). In addition, it was evident that stopped-flow experiments had to be conducted at low temperature $\left(4{ }^{\circ} \mathrm{C}\right)$ due to the high turnover rates of the enzyme (Wilkinson and Williams 1981).

Oxidized $E c \mathrm{E} 3\left(\mathrm{E}_{\mathrm{ox}}\right)$ has absorbance maxima at 369 and $455 \mathrm{~nm}$, a shoulder at $482 \mathrm{~nm}$, and minima at 312 and $395 \mathrm{~nm}$ (Figure 3.2A). Upon reduction with an equimolar amount of NADH the enzyme is transformed into its two-electron reduced form $\left(\mathrm{EH}_{2}\right)$ by intramolecular transfer of electrons from the reduced flavin to the disulfide. At the $\mathrm{EH}_{2}$ state the flavin remains oxidized while the redox active cystine is reduced. The flavin absorbance is attenuated and the entire spectrum is blue-shifted relative to the spectrum of $E_{\mathrm{ox}}$. In addition, the absorbance rises in the longer wavelengths region yielding in a shoulder at $523 \mathrm{~nm}$ which was attributed to the Cys50-thiolate FAD charge transfer complex (Thorpe and Williams 1976a, 1976b, 1981). This specific absorption change is based on an electron donoracceptor relation rather than on formation of a flavin radical species as demonstrated by independent experiments: At first, the complex shows clear $\mathrm{pH}$-dependent absorption changes yielding in a loss of the charge transfer signal at lower $\mathrm{pH}$ values which is indicative for protonation of the anionic electron donor (Matthews and Williams 1976). Secondly, the $\mathrm{EH}_{2}$ state was shown to be devoid of any detectable EPR signal (Massey and Gibson 1964; Searls, Peters, and Sanadi 1969) further 
underpinning the presence of a thiolate rather than a flavin radical giving rise to the absorption in the $523 \mathrm{~nm}$ region.

Flavin reduction and thiolate formation occur at the same apparent rate constant with $\sim 55 \mathrm{~s}^{-1}$ (Figure $3.2 \mathrm{C}$ and Figure 5.1). Though the flavin peak resides in its blue-shifted form during the recorded time the thiolate starts to decay. This is most likely due to the property of the $\mathrm{EH}_{2}$ state of the E. coli enzyme which is indeed a mixture of different spectrally distinct species (Wilkinson and Williams 1979). At the $\mathrm{pH}$ used for the experiments ( $\mathrm{pH}$ 7.6) it is possible that Cys50 becomes protonated and concomitantly the interchange thiol (Cys45) becomes deprotonated leading to a prototropic tautomer which explains the loss in absorbance at $523 \mathrm{~nm}$. Further reduction to the four-electron reduced state $\left(\mathrm{EH}_{4}\right)$ requires the flavin also being reduced. This is accompanied by reduction with a 5 -fold molar excess of NADH (Figure 3.2B). In the $\mathrm{EH}_{4}$ state the absorbance maxima are further reduced and the $523 \mathrm{~nm}$ charge transfer decayed entirely. Moreover, the excess of NADH enables the detection of the $\mathrm{FADH}_{2}-\mathrm{NAD}^{+}$intermediate giving rise in absorption between 600 and $700 \mathrm{~nm}$ (Argyrou et al. 2002). The latter complex can only be observed to minute amounts under equimolar conditions (Figure 3.2A). However, its formation is completed within the dead-time of mixing at both used substrate concentrations indicating the transience of the complex. The transience can be related to the redox potential of the flavin cofactor which is significantly more positive compared to the ones of pig heart and Mycobacterium tuberculosis lipoamide dehydrogenase, respectively (Argyrou et al. 2002; Matthews and Williams 1976; Wilkinson and Williams 1979). Noteworthy, no significant increase in absorbance at $380 \mathrm{~nm}$ was observed in the reaction of $\mathrm{E}_{\mathrm{ox}}$ with NADH under single turnover conditions (Figure 3.2D). The absence of an increase in absorbance at this wavelengths, that is typical for flavin-C4a adducts, suggests that the C4a-cysteinyl adducts does not accumulate to detectable amounts in the forward direction in $E c \mathrm{E} 3$ using NADH as reductant. The finding of this high instability of the intermediate is consistent with previously reported observations (Argyrou et al. 2002). It was suggested that this instability could arise if formation of the covalent adduct in both directions is considerably slower than its decay in both directions (Argyrou et al. 2002). Apparently, this also holds true for this central intermediate in $E c \mathrm{E} 3$.

In summary, the spectral properties of the three enzyme species detected are very similar to the spectra of the corresponding species of the pig heart and the aforementioned Mycobacterium tuberculosis lipoamide dehydrogenase (Argyrou et al. 2002; Matthews and Williams 1976). A transfer to the E. coli system was therefore successful and was thus used to study active site mutants to further investigate the reaction mechanism of $E c \mathrm{E} 3$ (3.3). A trustworthy approach to track the reductive half-reaction of $E c \mathrm{E} 3$, and thus the ability to test for the accumulation of the covalent $\mathrm{C} 4 \mathrm{a}$-flavin adduct in the forward direction, is presented in 3.2. 

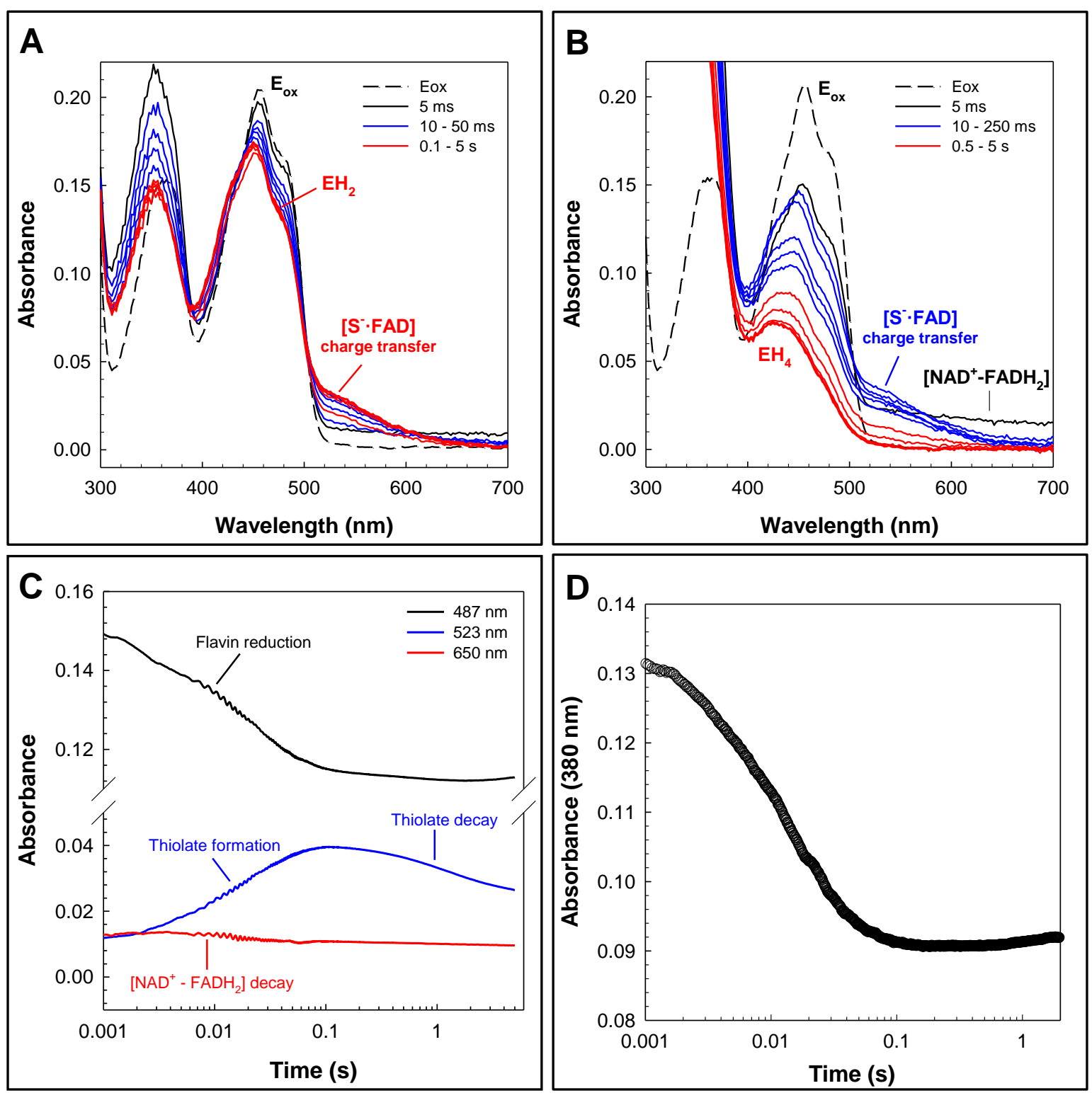

Figure 3.2 Stopped-flow absorbance traces of $E c \mathrm{E} 3$ during reduction with NADH.

$20 \mu \mathrm{M} E c \mathrm{E} 3$ were mixed with $20 \mu \mathrm{M}$ (A) or with $100 \mu \mathrm{M} \mathrm{NADH}(\mathbf{B})$ at $4{ }^{\circ} \mathrm{C}$ and $\mathrm{pH}$ 7.6. The dashed line represents the oxidized form of $E c \mathrm{E} 3\left(\mathrm{E}_{\mathrm{ox}}\right)$ derived from a single mixing event with buffer. The solid spectra were recorded using a diode-array detector after mixing with NADH at the concentrations indicated. Panel $\mathbf{C}$ indicates selected absorbance traces for the reduction under equimolar conditions. D Absorbance trace at $380 \mathrm{~nm}$ under single turnover conditions. No increase in absorbance can be detected indicating that the covalent $\mathrm{C} 4 \mathrm{a}$-cysteinyl adduct does not accumulate during the reduction process. Note the logarithmic time-scales. 


\subsubsection{Structure of EcE3 - Active Site Dynamics underlie Catalysis}

The crystal structure of $E c E 3$ was recently solved at medium resolution (2.5 $\AA$, PDB: 4JDR) (Chandrasekhar et al. 2013) and thus expanded the plethora of already determined structures of lipoamide dehydrogenases. The crystals belonged to space group I422 with two monomers assembled as functional dimer occupying the asymmetric unit. However, it was the aim this study to improve the existing structural data by means of resolution and model quality. An improved structural knowledge may help to gather insights in the transient nature of the enzyme's reaction cycle (3.1.2). Thus, a correlation of the structural findings with spectroscopic results in solution was applied whenever possible.

$E c \mathrm{E} 3 \mathrm{wt}$ could be crystallized in a new polymorphic form and the crystal structure was determined successfully with improved resolution and atomic model reliability as judged by refinement statistics (5.12). As described previously ammonium sulfate was used as precipitant but the crystallization condition harbored an increased $\mathrm{pH}$ (7.0). Crystals exhibited a shiny yellow color clearly indicating the presence of the flavin cofactor (Figure 3.4) and belonged to space group $\mathrm{P} 22_{1} 2_{1} 2_{1}$. Six monomers were present in the asymmetric unit corresponding to a solvent content of $59.99 \%$, labeled A - F, assembled into three functional dimers. The altered space group and the increased number of monomers occupying the asymmetric unit is likely due to the increased $\mathrm{pH}$ in the crystallization buffer which most likely influenced crystal packing. The structure of $E c \mathrm{E} 3$ was solved by molecular replacement with PHASER (McCoy et al. 2007) using a monomer of lipoamide dehydrogenase from Neisseria meningitidis as search model (PDB: 1OJT) and could be refined at $2.1 \AA$ resolution to $R_{\text {work }} / R_{\text {free }}$ of $17.7 / 20.8 \%$ (Table 5.3). After model completion and refinement, several difference map peaks ( $\mathrm{mFo}-\mathrm{DFc}$ at 3 sigma level) could be observed. Owing to the presence of sulfate in the crystallization condition and glycerol served as cryoprotectant, these peaks were modelled as sulfates and glycerols taking into account the character of interactions with surrounding residues. Interestingly, a sulfate acts as a placeholder molecule residing very close to the binding site for the diphosphate anchor of $\mathrm{NAD}^{+}$(Figure 5.22). At least 469 out of the 474 residues were visible in each of the six molecules occupying the asymmetric unit whereas the missing residues are housed in the C-terminal part are most likely disordered. The structure $E c E 3$ is similar to the already published one, for instance, dimer $\mathrm{AB}$ can be superimposed with an r.m.s.d. of $0.49 \AA$ calculated on $\mathrm{Ca}$ positions, indicates the high similarity.

Despite the high similarity with respect to the overall fold essential differences around the active site, particularly at the redox active cystine, could be observed. It was stated that the geometry of the redox active disulfide bridge in the $E$. coli enzyme deviates from the geometry observed in other organisms (Chandrasekhar et al. 2013). The dihedral angles (C $\beta-S \gamma-S \gamma-C \beta)$ were reported to be 29 and $46^{\circ}$ in one functional dimer. However, based on improved structural data presented here these results seem to be questionable. The dihedral angle in chain A was $-131^{\circ}$ and is thus consistent with earlier published 
geometries (Brautigam et al. 2005; Mande et al. 1996; Mattevi et al. 1991). Moreover, it was possible to model both an open and a closed conformation for the disulfide bridge contributing to the redox center (Figure 3.3A). Multiple refinements with different occupancies as starting point all converged into a defined model with a distribution of $50 \%$ open and $50 \%$ closed conformation for the cystine. Partial opening of the disulfide bridge is induced by the synchrotron radiation and leads to formation of two different redox states of $E c \mathrm{E} 3$ simultaneously. The closed conformation of the cystine resembles the oxidized state $\left(\mathrm{E}_{\mathrm{ox}}\right)$ and the open conformation resembles the two-electron reduced form $\left(\mathrm{EH}_{2}\right)$ of the enzyme. This radiation-induced reduction enabled the observation of structural rearrangements required for catalysis and gave first insights into the local plasticity of the active site. Further structural changes which are pivotal to stabilize the two-electron state of EcE3 and the formation of the covalent FAD-C4a-cysteinyl adduct are described below (3.1.4 and 3.1.5).

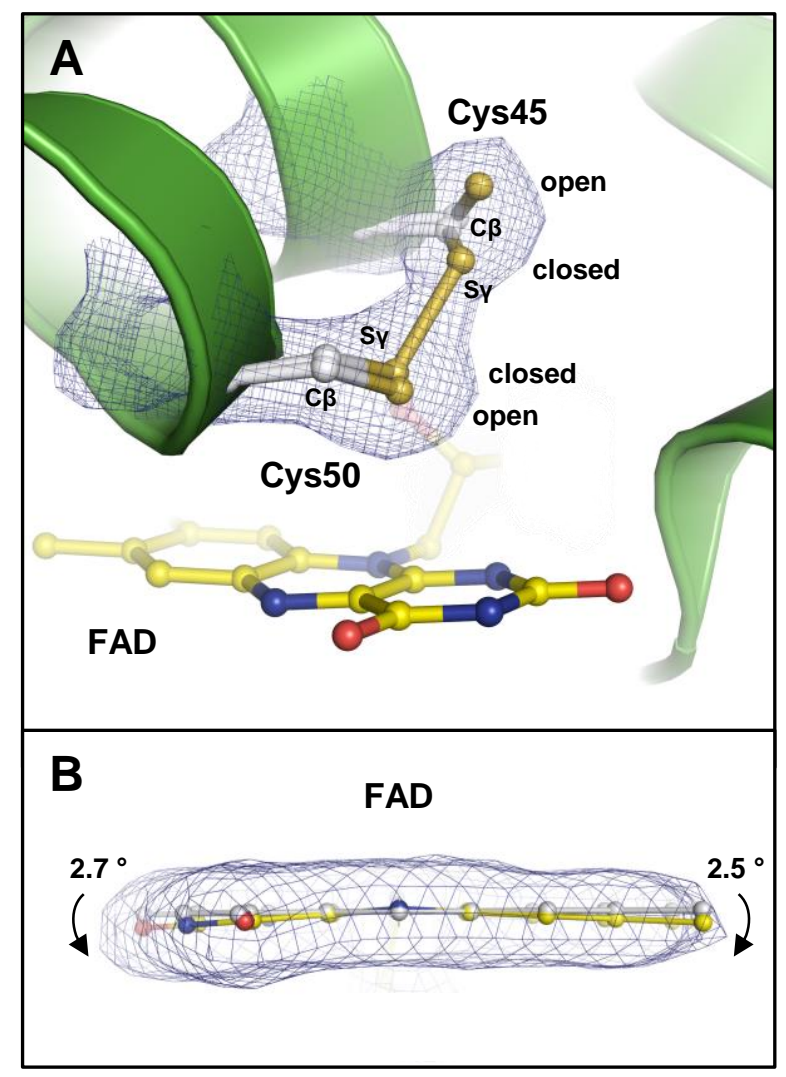

Figure 3.3 Local flexibility of the active site.

(A) The redox active disulfide bridge of $E c \mathrm{E} 3$ is formed between Cys45 and Cys50. Its open and closed conformation could be refined with $50 \%$ occupancy, respectively. The $2 \mathrm{mFo}-\mathrm{DFc}$ electron density map is countered at $2 \sigma$. The closed conformation reflects the oxidized, the open conformation the two-electron reduced state of EcE3.

(B) Deviation from planarity of the flavin cofactor. Superposition of the flavin cofactor from PDB: 4JDR (light grey) and $E c \mathrm{E} 3$ at $2.1 \AA$ (yellow). The pteridine part deviates $\sim 2.7$, the dimethylbenzene part $\sim 2.5^{\circ}$ from planarity ( $2 \mathrm{mFo}-\mathrm{DFc}$ map countered at $2 \sigma$ ).

The flexibility of the active site is not restricted to the redox active cystine. The aromatic isoalloxazine moiety of the flavin cofactor also deviates from planarity. The pteridine part is bent $\sim 2.7^{\circ}$, the dimethylbenzene part $\sim 2.5^{\circ}$ with respect to the N5-N10 axis of the cofactor (Figure 3.3B). It was demonstrated that the flavin redox potential can be tuned by conformational effects, especially by bending (Hasford et al. 1997; Lyubimov et al. 2007; Walsh and Miller 2003). Apparently, EcE3 also 
uses this tool maintaining the optimal redox potential. However, deviation from planarity is not as pronounced as observed in other studied flavoenzymes (Müller et al. 1994; Neumann et al. 2008).

\subsubsection{Spectroscopic and Crystallographic Investigation of the two-electron reduced State $-\mathbf{E H}_{2}$}

The two-electron reduced state $\left(\mathrm{EH}_{2}\right)$ of $E c \mathrm{E} 3$ is the catalytically active species that is formed prior to generation of NADH (3.1.2). During studies on the redox active disulfide it could be demonstrated that replacement of Cys45 to a serine residue $(E c \mathrm{E} 3 \mathrm{C} 45 \mathrm{~S})$ leads to a stable formation of an artificial $\mathrm{EH}_{2}$ state in which the remaining cysteine (Cys50) is present to $95 \%$ in its anionic form (Hopkins and Williams 1995a, 1995b). The residual $\sim 5 \%$ were estimated to be a fluorescent species in which Cys50 resides in its thiol form. $E c \mathrm{E} 3 \mathrm{C} 45 \mathrm{~S}$ harbored an orange-red color and its $\mathrm{UV}$-Vis spectrum resembled that of the two-electron reduced wild type enzyme. It is characterized by an $\sim 10 \mathrm{~nm}$ blue shifted flavin peak and the presence of an broad thiolate-FAD charge transfer absorbance between 500 to $600 \mathrm{~nm}$ (Figure 3.4A). The absence of an absorbance in the $380 \mathrm{~nm}$ range excluded the accumulation of a cysteinyl-C4a adduct in the resting state (Figure 3.4A, inset). It has been suggested that binding of oxidized pyridine nucleotides may facilitate flavin-disulfide interaction (Matthews et al. 1979). Therefore, $E c \mathrm{E} 3 \mathrm{C} 45 \mathrm{~S}$ was subjected to $\mathrm{NAD}^{+}$-titrations, which were carried out at $10{ }^{\circ} \mathrm{C}$ in order to slow down a putative FAD-C4a-cysteinyl adduct formation. However, even in the presence of the pyridine nucleotide a significant absorption increase in the characteristic wavelength region at $380 \mathrm{~nm}$ was not detectable (Figure 3.4B) and hence an $\mathrm{NAD}^{+}$-induced collapse between Cys50 and FAD-C4a is unlikely. The mechanism of cysteinyl-C4a adduct formation will be discussed below in detail (3.1.5 and 3.1.6).

To extend the understanding of how the two-electron reduced state of the E. coli lipoamide dehydrogenase is stabilized, $E c \mathrm{E} 3 \mathrm{C} 45 \mathrm{~S}$ was crystallized and the crystal structure was determined successfully. As for the wild type, the presence of ammonium sulfate as precipitant EcE3C45S was necessary. However, a lower $\mathrm{pH}$ value was required ( $\mathrm{pH} 6.5$ compared to $\mathrm{pH} 7.0$ ). The obtained crystals also showed a pale orange-red color indicating that the charge transfer interaction is still active in crystallo (Figure 3.4C). EcE3C45S crystals belonged to space group I422 and contained two monomers per asymmetric unit assembled into a functional homodimer. The structure was solved by molecular replacement using the ground state $E c \mathrm{E} 3$ structure as search model (3.1.3) was refined to $R_{\text {work }} / R_{\text {free }}$ of $23.3 \% / 27.4 \%$ against data to 2.50 Å resolution (Table 5.3). Difference map peaks (mFoDFc at 3 sigma level) of tetrahedral shape were modeled as sulfate ions as described for the ground state structure. 472 out of the 477 residues were traceable in both molecules whereas the residues resulting from cloning artefacts and the last $\mathrm{C}$-terminal residues could not be traced and are most likely disordered. The overall fold of EcE3C45S is similar to the ground state structure. Two catalytic 
functional dimers can be superimposed with an r.m.s.d. of $0.67 \AA$ calculated on $\mathrm{Ca}$ positions indicating the high similarity. However, slight differences can be observed which, are mainly located in the surface exposed regions of the $\mathrm{NAD}^{+}$- and FAD-binding domain whereas the enzyme's core regions are almost identical. Since the structural data of $E c \mathrm{E} 3 \mathrm{C} 45 \mathrm{~S}$ was of reasonable quality the resulting model resembles a snapshot of the two-electron reduced state of the enzyme and thus provides structural information about a central intermediate during catalysis. In this intermediate step the disulfide bridge is entirely broken and the cysteine residue facing the flavin cofactor is present as a thiolate (3.1.2). Major emphasis was therefore devoted to the hydrogen bond network at the active site which stabilizes the anionic form of Cys50. 

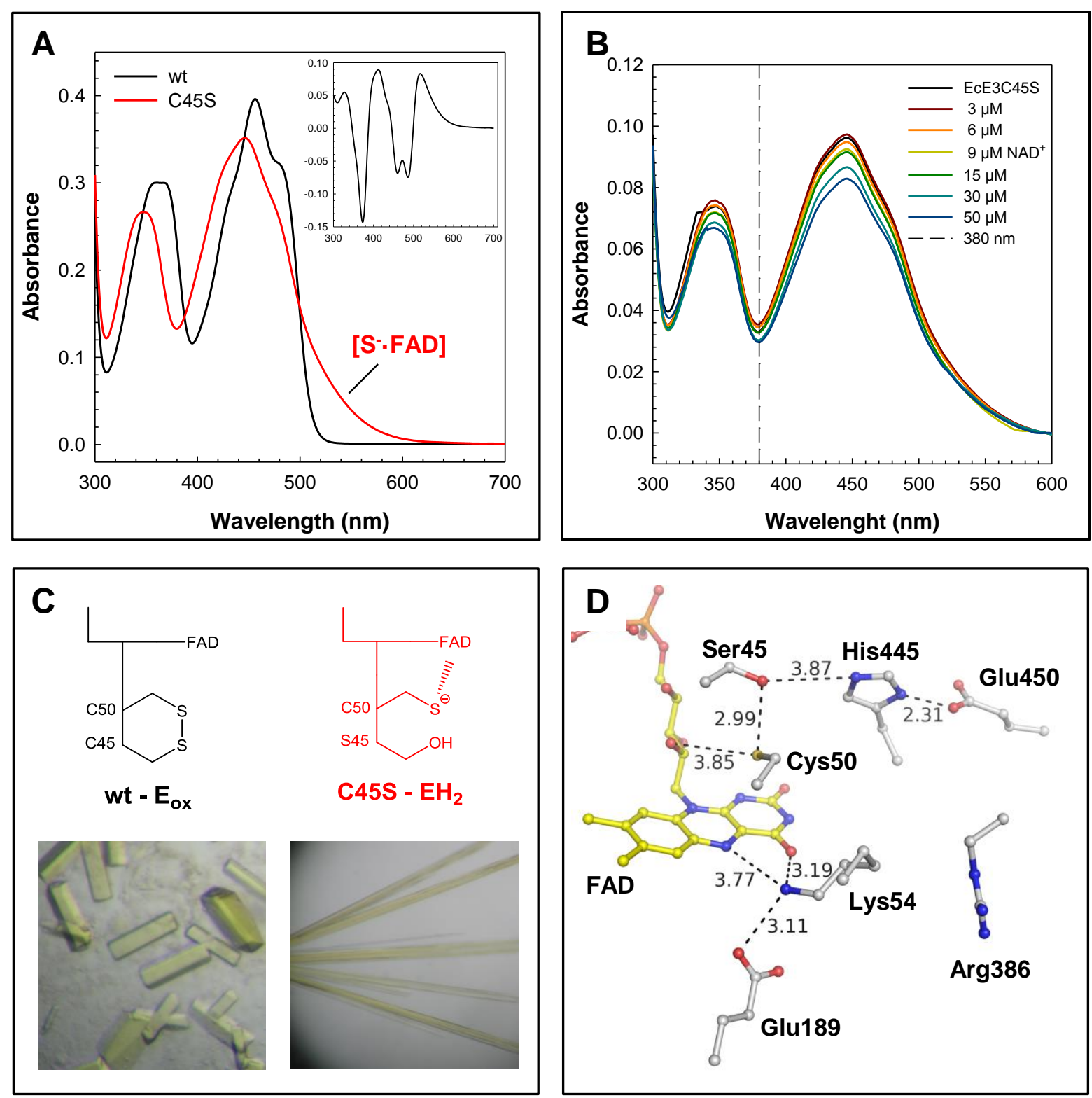

Figure 3.4 General characterization of $E c \mathrm{E} 3-\mathrm{EH}_{2}$.

(A) Resting state absorbance spectra of $E c \mathrm{E} 3 \mathrm{wt}$ and $\mathrm{C} 45 \mathrm{~S}$. The broad absorbance band between 500 and $600 \mathrm{~nm}$ indicates the charge transfer complex between the anionic Cys50 and FAD. (B) NAD ${ }^{+}$ titration of EcE3C45S at $\mathrm{pH}$ 7.6. Addition of $\mathrm{NAD}^{+}$does not lead to increase in absorbance at $380 \mathrm{~nm}$. An accumulation of the cysteinyl-C4a adduct is therefore unlikely. (C) Schematic of the active sites in $E c \mathrm{E} 3 \mathrm{wt}$ and $\mathrm{C} 45 \mathrm{~S}$ (top) and corresponding protein crystals. EcE3C45S retained its orange-red color in crystallo indicating that the charge transfer complex between Cys50 and FAD is still active. (D) Hydrogen bond network in $E c \mathrm{E} 3-\mathrm{EH}_{2}$. The thiolate of Cys50 is stabilized by a hydrogen bond to the $\mathrm{OH}$-group of Ser45 and the 2'-hydroxyl group of the ribityl chain (distances in $\AA$ ). 
A direct interaction of the Cys50-thiolate between the hydroxyl group of Ser45 and the 2'-hydroxyl of the ribityl side chain could be observed (Figure 3.4D). In addition, Ser45 might be stabilized by a polar interaction with His445. However, the mentioned polar interactions span distances in the range of $>3 \AA$ and are thus of a weak nature. Therefore, it is likely that additional elements contribute to thiolate stabilization (see discussion below). The $\varepsilon$-amino group of Lys54 is coordinated by the flavin cofactor as well as by Glu189. The $\mathrm{N} 5$ and $\mathrm{O}=4$ atom of the isoalloxazine form polar interactions to Lys54 as well as to the carboxylic function of the adjacent residue. Arg386, an active site nearby residue, is not involved in the hydrogen bond network. The role of the catalytic diad (His445 and Glu450) and of Lys54 and Arg386 during catalysis will be discussed later (3.3.1 and 3.3.2).

The stabilization of the thiolate in lipoamide dehydrogenases has been discussed controversially over the last decades. It was assumed that the imidazole of the conserved histidine directly functions as stabilizer of the thiolate (Kim and Patel 1992b; Matthews et al. 1979). The structural investigation of the ground state structure and of $E c \mathrm{E} 3 \mathrm{C} 45 \mathrm{~S}$ revealed that the $\mathrm{N} 3$ atom of His445 cannot donate a hydrogen bond to Cys50 (3.3.1.1). However, weak interaction is observed to Ser45 the major hydrogen bond donor to the thiolate attributing a more indirect role for His 445 during thiolate stabilization. The $\mathrm{pKa}$ for Cys50 was determined to be 2.7 and is thus relatively low compared to other cystine containing oxidoreductases (Hopkins and Williams 1995a). This denotes that the particular milieu of the redox active cystine is pivotal for the acidity of the respective thiol. Besides the hydrogen bond network described above further factors may contribute to thiolate stabilization and the lowering of the $\mathrm{pKa}$ value. At first, the redox active cystine is part of a long alpha helix belonging to the FADbinding domain located in the core of the catalytic unit (3.1.3). It was suggested that the positive end originating from such helix dipole might contribute to stabilization of the thiolate (Benen et al. 1991). Secondly, $\pi$-stacking interactions between the electron-rich sulfur and the electron-deficient FAD cofactor also may contribute to stabilization implying that cofactor and cysteine residue truly act as an electron-donor-acceptor complex. The concomitant alteration in the electronic property of the FAD is underpinned by a further bending of the cofactor compared to the ground state structure (Figure 5.23).

In summary, the crystal structure analysis of the two-electron reduced state of $E c \mathrm{E} 3$ revealed that the catalytic role of the interchange thiol (Cys45) has to be expanded. So far, this residue was stated to be solely important with regard to bond formation with the substrate dihydrolipoamide forming a mixed disulfide and the subsequent electron transfer to Cys50 yielding the charge transfer complex. However, Cys45 also stabilizes the latter complex by a hydrogen bond contributing directly to a further central catalytic state. Moreover, the results obtained allow the prediction that Cys45 is protonated during this state of catalysis in the wild type enzyme. 


\subsubsection{Spectral Evidence for the Cysteinyl-C4a Adduct in a monothiol Variant of EcE3}

Rapid reaction experiments showed that the covalent $\mathrm{C} 4 \mathrm{a}$-flavin adduct is highly instable leading to a limited accumulation which make this central intermediate undetectable during the transfer of electrons from the reduced flavin to the redox active cystine (3.1.2). These results are consistent with previously published data for the lipoamide dehydrogenase from Mycobacterium tuberculosis (Argyrou et al. 2002). Further, a direct kinetic observation of the intermediate during catalysis has never been possible. The putative accumulation from the opposite direction, transfer from the reduced disulfide to the oxidized flavin is discussed in 3.2.3. Thus far, the C4a-cysteinyl adduct has only been observed under non-physiological conditions using several enzymes belonging to the class of flavindependent oxidoreductases. At first, in monoalkylated pig heart lipoamide dehydrogenase upon addition of $\mathrm{NAD}^{+}$(Thorpe and Williams 1976b, 1981), secondly, in the two electron reduced thioredoxin reductase substituted with 1-deaza-FAD at low pH (O'Donnell and Williams 1984), and thirdly, also at low pH in mercuric reductase upon rapid mixing with NADPH (Sahlman, Lambeir, and Lindskog 1986). In the latter three cases, the spectrum of the adduct was estimated by extrapolation. However, there was also direct spectral evidence for the $\mathrm{C} 4 \mathrm{a}$ adduct, when an oxidized active site triple variant (three out of the four catalytic cysteines were replaced by an alanine, ACAA mutant) of mercuric ion reductase was titrated with $\mathrm{NADP}^{+}$also at low $\mathrm{pH}$ (Miller et al. 1990).

Therefore, a monothiol variant $(E c \mathrm{E} 3 \mathrm{C} 45 \mathrm{~A})$ was generated in which the interchange thiol forming the mixed disulfide during catalysis was replaced by an alanine. This catalytically almost inactive variant (5.4) was then subjected to $\mathrm{NAD}^{+}$titrations at lower $\mathrm{pH}$ values since it is apparent that both lower $\mathrm{pH}$ values and the presence of the pyridine nucleotide facilitate adduct formation. The UV-Vis ground state spectrum of $E c \mathrm{E} 3 \mathrm{C} 45 \mathrm{~A}$ is clearly different from that of the wild type enzyme (5.2B). The spectrum shows a hypsochromic shift yielding in a $\lambda_{\max }=448 \mathrm{~nm}$, a way less pronounced shoulder in the $470 \mathrm{~nm}$ region and a very small absorbance between 500 and $700 \mathrm{~nm}$. Interestingly, the spectral property is congruent with the spectrum of the aforementioned lipoamide dehydrogenase from pig heart in which the interchange thiol had been alkylated (Thorpe and Williams 1976b). No indicative band in the range of $380 \mathrm{~nm}$ is present in the difference spectrum suggesting that the remaining cysteine residue is not bound to the cofactor per se. (5.2B, inset). A comparison with the $\mathrm{EH}_{2}$ mimic $E c \mathrm{E} 3 \mathrm{C} 45 \mathrm{~S}$ leads to the assumption that Cys50 is mostly present in its thiol form since the typical charge transfer band in the $530 \mathrm{~nm}$ region indicating the thiolate-FAD interaction is missing. The fact that the main hydrogen bond donor - Cys45 during the $\mathrm{EH}_{2}$ state - stabilizing the anionic form of Cys50 was removed further underpins this assumption (3.1.4). Moreover, it was demonstrated that replacement of active sites cysteine residues with alanines in mercuric ion reductase increased the hydrophobicity and thus consequently raised the $\mathrm{pKa}$ leading to formation of the thiol form. (Miller et al. 1990). 

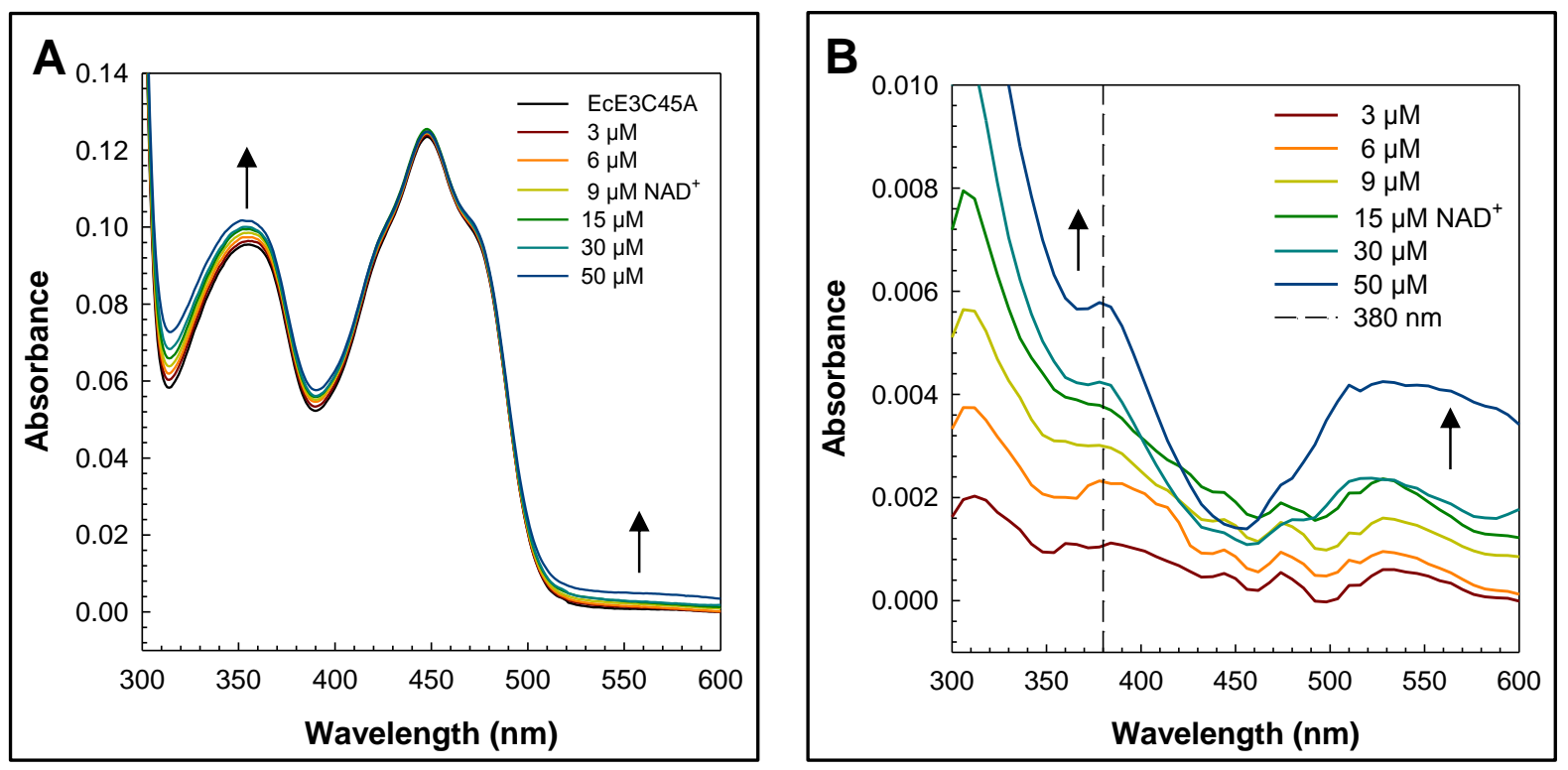

Figure 3.5 $\mathrm{NAD}^{+}$titration of $E c \mathrm{E} 3 \mathrm{C} 45 \mathrm{~A}$.

(A) Lower temperature $\mathrm{NAD}^{+}$titration at $10{ }^{\circ} \mathrm{C}$ and in $100 \mathrm{mM}$ MES pH 6.0. Binding of $\mathrm{NAD}^{+}$leads to increase in absorbance between $300-400 \mathrm{~nm}$ and between $500-600 \mathrm{~nm}$, respectively. (B) Difference spectra of the same titration. NAD ${ }^{+}$induces a band formation peaking at $380 \mathrm{~nm}$ with a concomitant broad increase in absorbance from 500 to $600 \mathrm{~nm}$. Difference spectra were smoothed according to a bisquare weighting technique and a polynomial regression. The experiments were performed in duplicates.

Addition of $\mathrm{NAD}^{+}$led to small changes in the spectrum of $E c \mathrm{E} 3 \mathrm{C} 45 \mathrm{~A}$ (Figure 3.5A). An increase in absorbance occurs from 300 to $400 \mathrm{~nm}$ with a concomitant smaller increase from 500 to $600 \mathrm{~nm}$. However, the changes in absorption are not as pronounced as compared to the changes observed in the mercuric reductase ACAA mutant where a loss of the typical oxidized flavin absorption was observed (Miller et al. 1990). However, the generated difference spectra clearly indicated the presence of a band peaking at $380 \mathrm{~nm}$ with increasing $\mathrm{NAD}^{+}$concentrations albeit with a small amplitude (Figure 3.5B). This increase is accompanied with a quenching of the flavin peak around $450 \mathrm{~nm}$ and a more pronounced increase in the red part of the spectrum. Strikingly, comparable spectral changes could be observed at $\mathrm{pH} 8.3$ (Figure 5.2) indicating that the spectral changes also occur in a more basic environment. $\mathrm{NAD}^{+}$titrations under the same conditions for the wild type enzyme did not lead to any increase throughout the entire spectrum, solely the quenching in the $450 \mathrm{~nm}$ region was visible (Figure $5.3)$.

The obtained spectra suggest that a covalent adduct populated in minor fractions since the rise in absorption at $380 \mathrm{~nm}$ is completely consistent with the formation of FAD-C4a-adducts (Ghisla et al. 1974, 1977). Further, the necessity for $\mathrm{NAD}^{+}$binding to induce adduct formation is consistent with previous studies (Matthews et al. 1979; Miller et al. 1990; Thorpe and Williams 1976b, 1981). It was 
suggested that binding of the positively charged nucleotide at the $r e$ face of the flavin increases the electrophilicity of the oxidized flavin, thereby promoting C4a-cysteinyl formation (Miller et al. 1990). However, recent results generally demonstrated that structural perturbations at the active site seem to be essential to close internuclear distances (Klinman 2009, 2010). The role of $\mathrm{NAD}^{+}$during catalysis might therefore be expanded. Given the inevitable presence of $\mathrm{NAD}^{+}$to drive adduct formation combined with the structural geometry of the FAD-C4a-cysteinyl bond (3.1.6), it is not unlikely that the pyridine nucleotide permits an active site compression which is pivotal for the enzyme's transient catalysis.

Formation of the $\mathrm{C} 4 \mathrm{a}$-adduct in the $E c \mathrm{E} 3$ monothiol variant was possible at both low and high $\mathrm{pH}$. It is therefore likely that the remaining thiol delivers the proton required for N5 protonation during FADC4a-cysteinyl adduct formation. For mercuric reductase ACAA mutant it was suggested that this process might be assisted by polarization of the thiol by general-base catalysis (Miller et al. 1990), however the residue qualified for this polarization (Tyr441) turned out to be not correctly positioned (Ledwidge et al. 2005). Further, the distance of the $E c \mathrm{E} 3$ active site His445 is too long for a direct interaction with the adduct forming thiol based on the information provided by the ground state structure (Figure 3.15). It is therefore likely that a general base catalysis is not required to drive a thiol-based adduct formation so that the increased electrophilicity of the oxidized flavin upon $\mathrm{NAD}^{+}$ binding is sufficient to promote the collapse. 


\subsubsection{Crystallographic detection of the Cysteinyl-C4a Adduct}

During the course of this study it was possible to gather structural information of two distinct catalytic states of the $E c \mathrm{E} 3$ reaction cycle. Thereby, the ground state structure represents the oxidized state of the enzyme (3.1.3) and the structure of $E c \mathrm{E} 3 \mathrm{C} 45 \mathrm{~S}$ mimics the two-electron reduced state (3.1.4). Moreover, the ground state structure also demonstrated the local flexibility of the active site due to opening of the redox active disulfide bridge by radiation damage during data collection. Given the information that the synchrotron X-ray radiation can partially alter the enzyme's redox state this radiation was used to trigger the formation of the covalent FAD-C4a-cysteinyl adduct in crystallo. Two prerequisites were therefore necessary, at first, a further accumulation of the open state of the reactive cysteines and, secondly, the presence of $\mathrm{NAD}^{+}$in the crystal at the time-point of the data collection since the nucleotide is thought to facilitate adduct formation. The necessity of the presence of $\mathrm{NAD}^{+}$to induce adduct formation was further discussed in 3.1.5.

A single wild type crystal was therefore soaked with $5 \mathrm{mM} \mathrm{NAD}^{+}$during the cryo protection step and the structure was determined using the wild type structure as the starting model (3.1.3). The six monomers present in the asymmetric unit were accordingly labeled $\mathrm{A}-\mathrm{F}$. The structure was refined to $R_{\text {work }} / R_{\text {free }}$ of $17.6 / 22.1 \%$ against data to $2.6 \AA$ resolution using NCS restraints (Table 5.3). Difference map peaks ( $\mathrm{mFo}-\mathrm{DFc}$ at 3 sigma level) which was not related to the protein or water molecules after structure refinement were modelled as described above. At least 469 out of the 474 residues were visible in each of the six molecules occupying the asymmetric unit whereas the missing residues are housed in the C-terminal part, a fact which was also observed in the structure resolved at higher resolution. Despite the reasonable quality of the structure no clear evidence for presence of an $\mathrm{NAD}^{+}$ molecule at the re face of the flavin cofactor was found. It was demonstrated that $\mathrm{NAD}^{+}$bound to human lipoamide dehydrogenase can adopt different conformations at the binding site (Brautigam et al. 2005) explaining a certain flexibility of this ligand-enzyme interaction. Moreover, the usage of a highly concentrated sulfate solution as precipitant (2.4 M compared to $\left.5 \mathrm{mM} \mathrm{NAD}^{+}\right)$intrinsically leads to a higher occupancy of the precipitant at the diphosphate anchor binding site prohibiting a higher population for $\mathrm{NAD}^{+}$(Figure 5.22). However, the usage of higher concentrations of the pyridine nucleotide during the phase of cryo protection led to deterioration of the diffraction properties of the crystals and was therefore not taken into consideration for further experiments.

Occupancy refinements as conducted for the ground state structure revealed that the catalytic disulfide resided to $\sim 90 \%$ in the open conformation. The first requirement, a further radiation-induced reduction of the catalytic cystine, was therefore fulfilled. Moreover, during the phase of model improvement cohesive electron density between Cys50 and the $\mathrm{C} 4 \mathrm{a}$ atom of the flavin cofactor could be observed in subunits $\mathrm{A}$ and $\mathrm{F}$ in the $2 \mathrm{mFo}-\mathrm{DFc}$ density map. To further elucidate the nature of this density $2 \mathrm{mFo}-\mathrm{DFc}$ and $\mathrm{mFo}-\mathrm{DFc}$ difference maps were calculated using CNS program (Brunger 2007). This analysis revealed the presence of positive difference peaks near Cys50 pointing towards 
FAD-C4a (Figure 3.6A) in all EcE3 molecules present in the asymmetric unit. Since the default settings of the refinement program did not allow a further converging of Cys50 towards the flavin due to repulsive interactions the flavins were flagged as alternate conformations corresponding to Cys50 in open and closed state, which resulted in overcoming the restriction. Subsequent refinement led to a further convergence of Cys50 towards the positive difference peaks and thus the cofactor (Figure $3.6 \mathrm{~A})$.
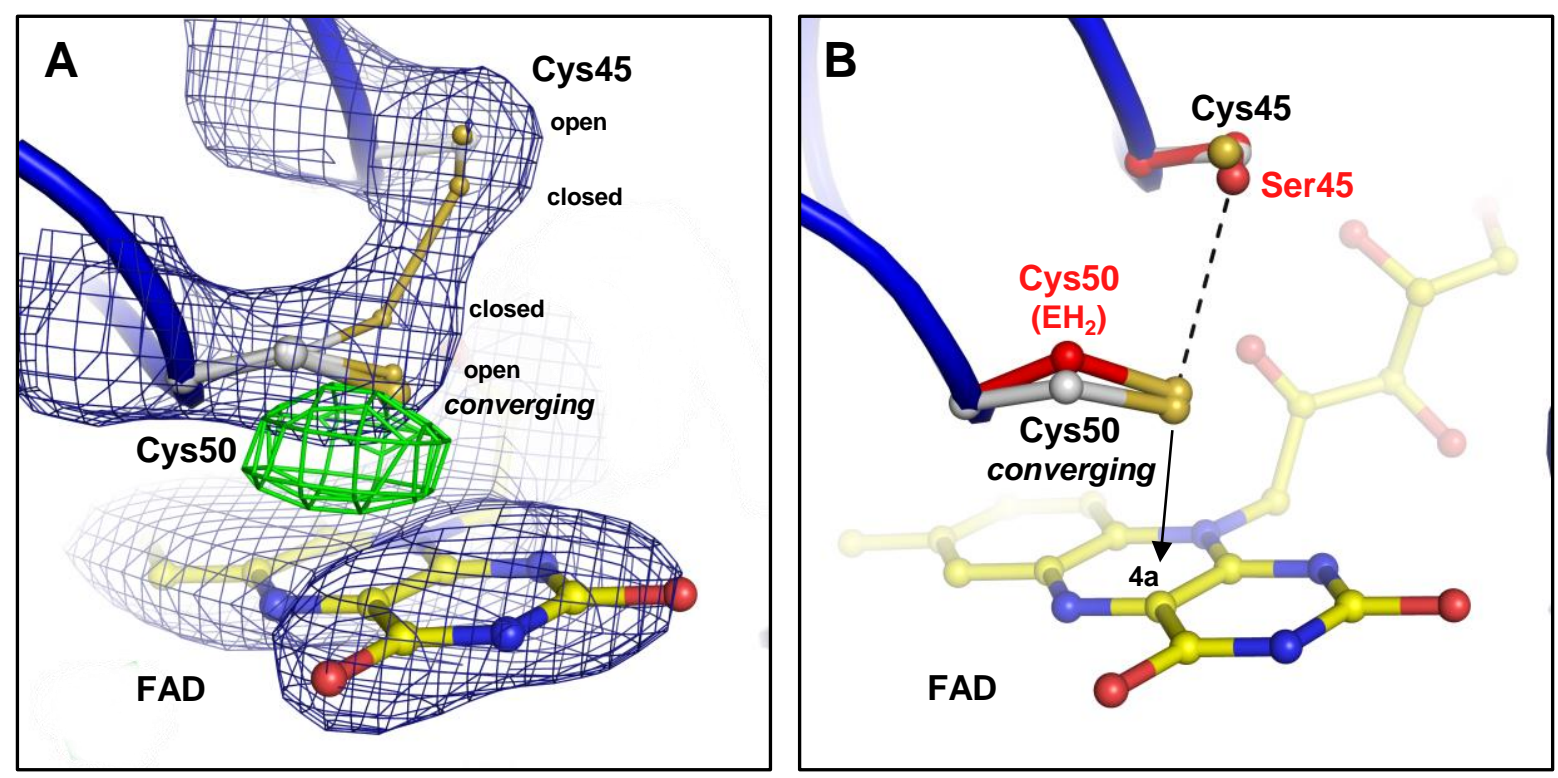

Figure 3.6 The flexibility of cysteine 50 is determined by its hydrogen bond donor.

(A) Convergence of Cys50 towards FAD-C4a. CNS calculated 2mFo-DFc map countered at $2 \sigma$ (blue mesh) and mFo-DFc difference map countered at $3 \sigma$ (green mesh) in molecule A. Applying an alternate conformation of FAD allowed a further convergence of Cys50 towards FAD since repulsive interactions restraints were overcome. The converging conformer of Cys50 is displayed by an enlarged ball and stick representation. Residual five molecules in the asu showed comparable difference peaks.

(B) Superposition of the catalytic cystine of the EcE3-NAD structure (light grey) with the $\mathrm{EH}_{2}$ state of $E c \mathrm{E} 3 \mathrm{C} 45 \mathrm{~S}$ (red). In $E c \mathrm{E} 3 \mathrm{C} 45 \mathrm{~S}$ a further migration towards $\mathrm{FAD}-\mathrm{C} 4 \mathrm{a}$ is prevented. The hydrogen bond between Ser45 and Cys50 is indicated by a dashed line. For convenience the closed conformation of the $E c \mathrm{E} 3-\mathrm{NAD}$ structure is not shown.

A comparison with the structure of the two-electron reduced enzyme $(E c \mathrm{E} 3 \mathrm{C} 45 \mathrm{~S})$ revealed that the converged Cys50 residue is in lower distance to the $\mathrm{C} 4 \mathrm{a}$ atom of the cofactor (Figure 3.6B). Multiple rounds of PHENIX refinements with the same parameters, which led to convergence of Cys50 in the $\mathrm{E} 3-\mathrm{NAD}^{+}$structure, confirmed that the anionic $\mathrm{Cys} 50$ of the $\mathrm{EH}_{2}$ structure resided at its position. This result is consistent with in solution experiments which showed that $\mathrm{C} 4 \mathrm{a}-$ cysteinyl adduct formation 
$E c \mathrm{E} 3 \mathrm{C} 45 \mathrm{~S}$ is impeded (Figure 3.4). In addition, this impairment is further confirmed by a significantly reduced enzyme activity compared to the wild type enzyme (5.4). The structural and electronic properties leading to this inertance of the thiolate in the variant have been discussed in 3.1.4. Though the convergence of Cys50 could be proven an appropriate model building was not possible since the difference map peak were at a position within the B-factor spread of the open conformation.

To rule out that the additional density was only based on elevated atomic displacement parameters and was thus model biased the possible presence of a covalent conjugate between Cys50 and FAD-C4a was investigated by the generation of simulated-annealing OMIT maps in which both the flavin cofactor and the cystine were omitted. The calculated model-bias free mFo-DFc electron density map revealed the presence of prominent cohesive density between cysteine50 and the $\mathrm{C} 4 \mathrm{a}$ atom of the flavin in molecule $\mathrm{A}$ and molecule $\mathrm{F}$ of the asymmetric unit and thus the likely presence of a populated covalent FAD-C4a-cysteinyl adduct (Figure 3.7).

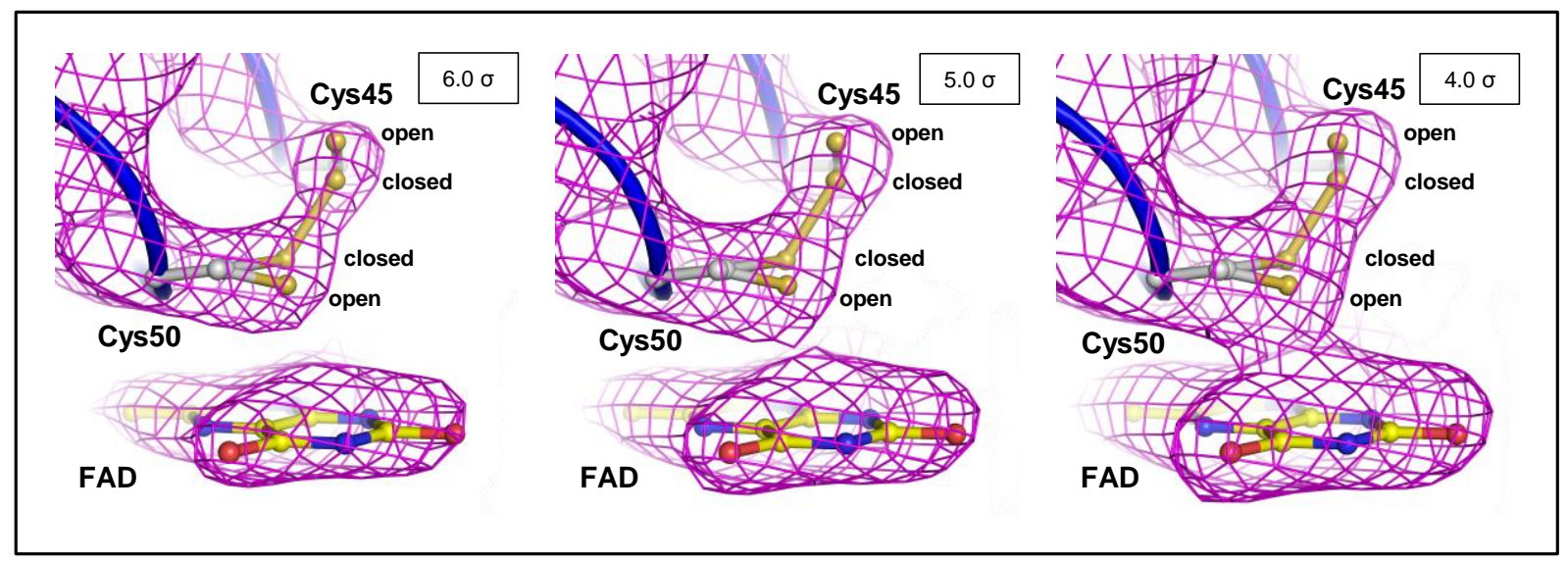

Figure 3.7 Evidence for cohesive electron density between Cys50 and FAD-C4a.

The presence of cohesive density implicating a covalent linkage between Cys50 and FAD-C4a is supported by an $\mathrm{mFo}-\mathrm{DFc}$ simulated-annealing OMIT map displayed at different sigma levels (magenta mesh) for one of the active sites (monomer F). Note that with decreasing sigma level the electron density converges from both omitted parts, the cystine and the FAD. An overview of SA OMIT maps for all molecules in the asymmetric unit countered at equal sigma level is shown in the appendix (Figure 5.25).

Two aspects have to be considered underpinning the likely presence of a covalent linkage between Cys50 and FAD-C4a. At first, the density approaches from both interacting partners towards each other, from the cysteine as well as the FAD. Thus, the congruent density is not only based on a highly mobile cysteine further migrating towards the cofactor but also on structural rearrangements at the flavin site presumably the formation of a tetrahedral C4a atom pointing towards Cys50. Secondly, the 
atomic displacement parameters of the cofactor and the redox active cystine are not significantly elevated compared to the residues around the active site (Figure 5.24). Taking these considerations into account an appropriate model building of the covalent adduct was possible.

The electron density allowed the modelling of additional conformations of both the flavin and Cys50. An additional side chain rotamer for Cys50 (50\% probability) taken from the Coot side chain library (Emsley et al. 2010) suitable fitted into the SA OMIT maps without the need of further adjustments (Figure 3.8). A covalent adduct at the $\mathrm{C} 4 \mathrm{a}$ position of the flavin requires a tetrahedral geometry of this part of the isoalloxazine moiety. Therefore, a tetrahedral FAD with the $\mathrm{C} 4 \mathrm{a}$ atom pointing towards Cys50 was modeled in the OMIT density using a rigid body fit. This fit implicated a restrain concerning the bond length between the $\mathrm{C} 4 \mathrm{a}$ and sulfur atom of Cys50 which was set to $1.81 \AA$, a typical length for a C-S bond (Figure 3.8). The resulting overall model adequately fitted into calculated OMIT maps and thus likely reflects the crystallographic detection of the FAD-C4acysteinyl adduct which has been predicted to exist but an experimental proof was thus far missing. This study supports the existence of this long-sought-after intermediate not only by UV-Vis spectroscopic studies (3.1.5) but further underpins its existence by means of X-ray crystallography.

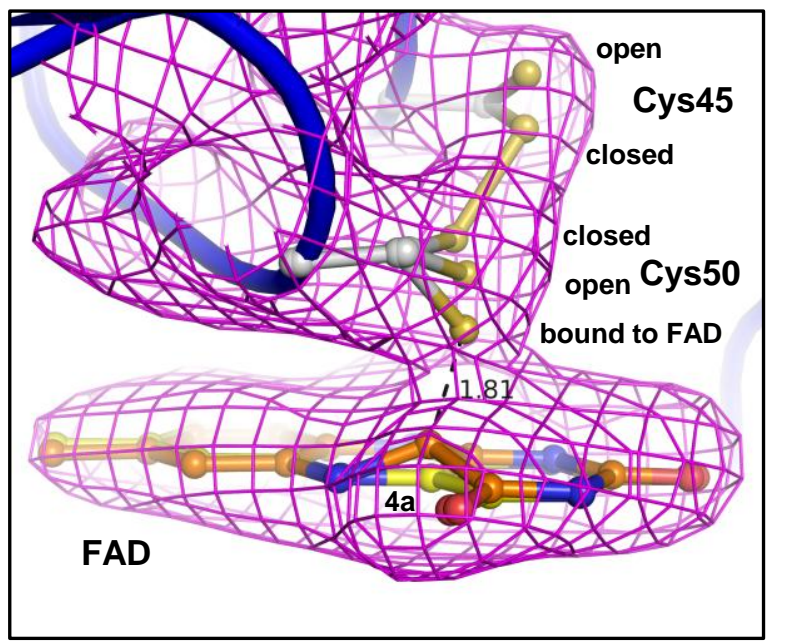

Figure 3.8 Structural model of the covalent C4acysteinyl adduct.

The model represents an additional redox state of $E c \mathrm{E} 3$. Besides the previously described open and closed conformation of the redox active cystine the modelling of the conformer which is bound to the FAD is possible. The tetrahedral FAD was modelled into the mFo-DFc SA-OMIT map (countered at $4 \sigma$; magenta mesh) using a rigid body fit implying a bond length of $1.81 \AA$ a between $\mathrm{C} 4 \mathrm{a}$ and $\mathrm{S} \gamma$ of Cys50.

\subsubsection{Is the geometry of the FAD-C4a-cysteinyl adduct the fundament for its transience?}

Given the spectroscopic and structural information about the transient nature of the FAD-C4acysteinyl adduct in $E c \mathrm{E} 3$ a comparison with this type of adduct present in LOV domains is possible. In 
solution data suggested that the C4a-cysteinyl adduct only accumulates to a minor extent (3.1.5). In addition, a direct spectroscopic observation during the reaction cycle was also not possible, neither with the oxidative half-reaction (NADH as reductant) nor with the reductive half-reaction $\left(\mathrm{Lip} 3 \mathrm{H}_{2}\right.$ as reductant) as starting point (3.1.2 and 3.2.3). The half-life was estimated to be $<2 \mathrm{~ms}$ and the decay is thus at least 250-fold faster compared to C4a-cysteinyl adducts in LOV domains. Here, the thermally driven dark state recovery is generally larger than $500 \mathrm{~ms}$ but can also last hours (Conrad et al. 2014). The decay in LOV domains is modulated by numerous of effects like the accessibility of solvent towards the active site, the electronic environment of the flavin and the hydrogen bonding network to the cofactor (Losi and Gärtner 2011, 2012; Zoltowski et al. 2009). Moreover, the presence of a base also has the ability to modulate adduct decay (Alexandre et al. 2007). The differences in the hydrogen bonding network of $E c \mathrm{E} 3$ compared to LOV domains around the flavin cofactor will be discussed later (3.4.4).

Besides this plethora of key modulators, a geometrical aspect was also taken into account acting as driving force responsible for adduct decay. The energy content of the adduct state in LOV domains was estimated to be $110-140 \mathrm{~kJ} \mathrm{~mol}^{-1}$ indicating a strained protein conformation (Losi, Kottke, and Hegemann 2004; Losi, Quest, and Gärtner 2003). These results are in slight disagreement with X-ray crystallographic data (Crosson and Moffat 2001, 2002). Dark and lit state structures revealed that only minor rearrangements in the protein are required to induce both adduct formation and decay. Indeed, the structural changes seem to be limited to the FMN on the one, and the bond forming cysteine on the other hand yielding a tetrahedral C4a-cysteinyl adduct (Crosson and Moffat 2002) (Figure 3.9A). The small a-helix of which the bond-forming cysteine is part of is positioned in a way that a simple rotamer movement of the latter cysteine is sufficient to fulfill adduct formation. On the contrary, the redox active cystine of $E c \mathrm{E} 3$ is part of a long a-helix protruding through the entire $E c \mathrm{E} 3$ monomer which does not allow the formation of relaxed tetrahedral flavin-cysteinyl adducts. A more detailed comparison of these structural data revealed that the adduct in $E c \mathrm{E} 3$ deviates from tetrahedral geometry harboring a $\mathrm{C} \beta-\mathrm{S} \gamma-\mathrm{C} 4 \mathrm{a}$ angle of $>117^{\circ}$ (Figure 3.9B). Since geometrical aspects have been shown to be crucial to enhance enzyme catalysis (Lüdtke et al. 2013; Tittmann 2014) it is possible that this aspect also have to be taken into account for lipoamide dehydrogenases. It is therefore most likely that the high transience of the adduct is, at first, based on its distorted geometry of the C4a-cyteinyl adduct and secondly based on the necessity of the presence of $\mathrm{NAD}^{+}$which is required per se to facilitate adduct formation (3.1.5). 


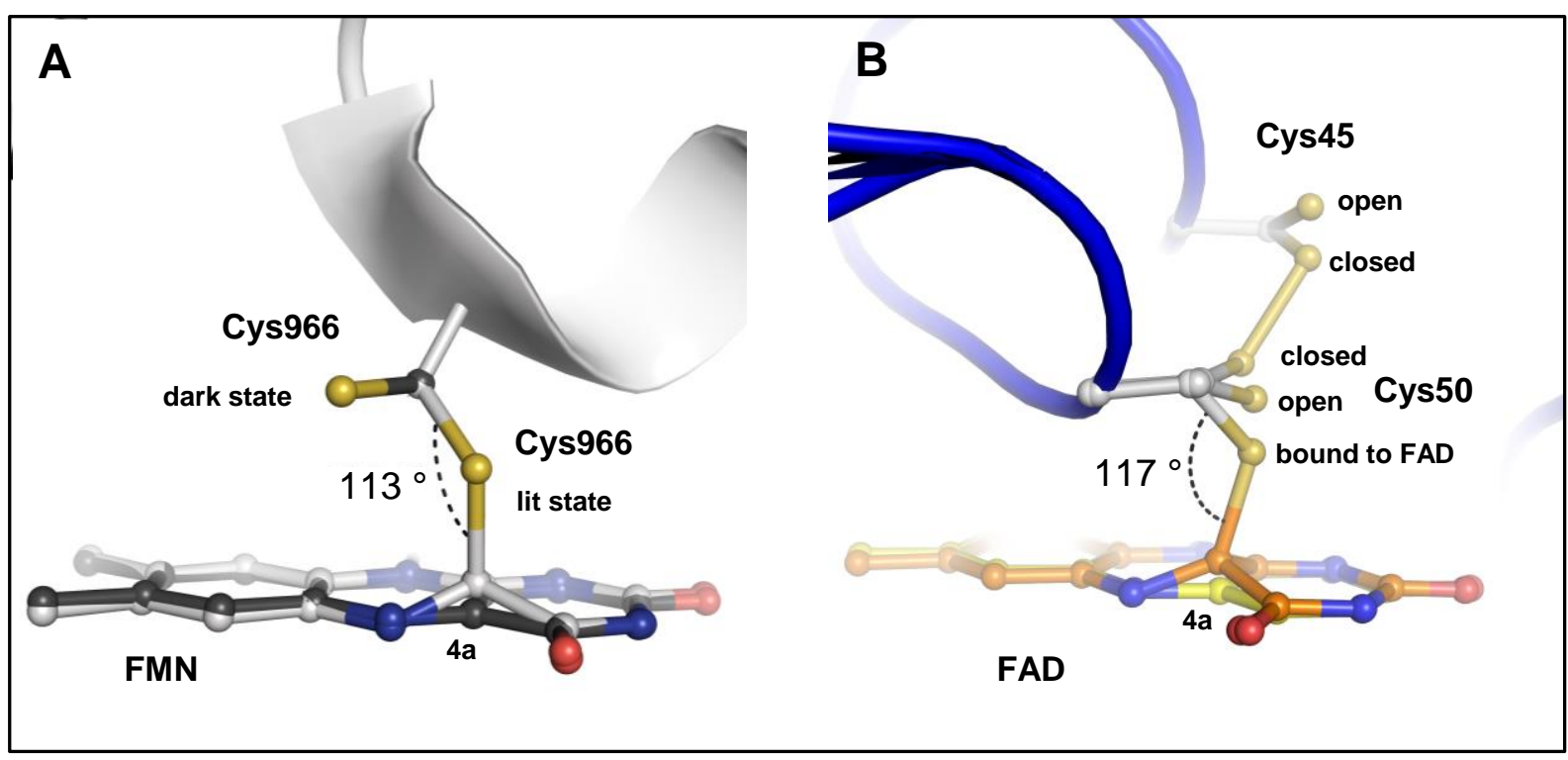

Figure 3.9 Comparison of the geometries of C4a-cysteinyl adducts in LOV domains and in EcE3.

(A) Superposition of the dark (dark grey) and lit state (light grey) of Adiantum LOV2 (PDB: 1G28 and $1 \mathrm{JNU}$ ). The angle of $113^{\circ}$ between $\mathrm{C} \beta-\mathrm{S} \gamma-\mathrm{C} 4 \mathrm{a}$ allowed a tetrahedral modelling. (B) Structural model of the covalent adduct in $E c \mathrm{E} 3$. The $\mathrm{C} \beta-\mathrm{S} \gamma-\mathrm{C} 4 \mathrm{a}$ angle of $>117^{\circ}$ reveals an extended deviation from tetrahedral geometry.

\subsection{Investigation of the reductive half-reaction of $E c E 3$ using $E c L i p 3$ as native Substrate}

The assignment of different reaction intermediates starting from the oxidative half-reaction with NADH as reductant was successfully established (3.1.2). To further investigate the reaction mechanism of $E c \mathrm{E} 3$ the isolated reductive half-reaction was also subjected to stopped-flow absorbance spectroscopy. The native substrate of lipoamide dehydrogenases are the reduced lipoamide arms of the E2 component of several multienzyme complexes. Thus, the most elementary in vitro substrates would be D,L-dihydrolipoamide or D,L-dihydrolipoate. However, these substrates show high $K_{\mathrm{M}}$ values, and in addition, D,L-lipoamide has limited solubility in aqueous solutions (2.2.4.1) (Argyrou and Blanchard 2001). There has been effort to mimic the physiological substrate more closely thereby improving substrate affinity and water solubility by the synthesis of D,L-lipoylbutanoate and D,Llipoylpentanoate (Argyrou et al. 2003). Both artificial substrates, containing an extended lipoic acid arm, with three and four additional methylene groups, respectively, were used successfully as substrates after reduction with borohydride yielding the respective dihydro-form. Significant higher $k_{\text {cat }} / K_{\mathrm{M}}$ values for both synthesized substrates relative to that of D,L-lipoamide reflected the increased specificity (Argyrou et al. 2003). However, transient kinetic and spectroscopic data with the 
physiological substrate as reductant are missing. Therefore, the entire third lipoyl domain of the E. coli dihydrolipoyl transacetylase $(E c E 2)$ was isolated, transferred in its dihydro-form under physiological conditions and used as substrate during both stopped-flow and sequential stopped-flow absorbance spectroscopy. The isolated lipoyl domain was termed EcLip3.

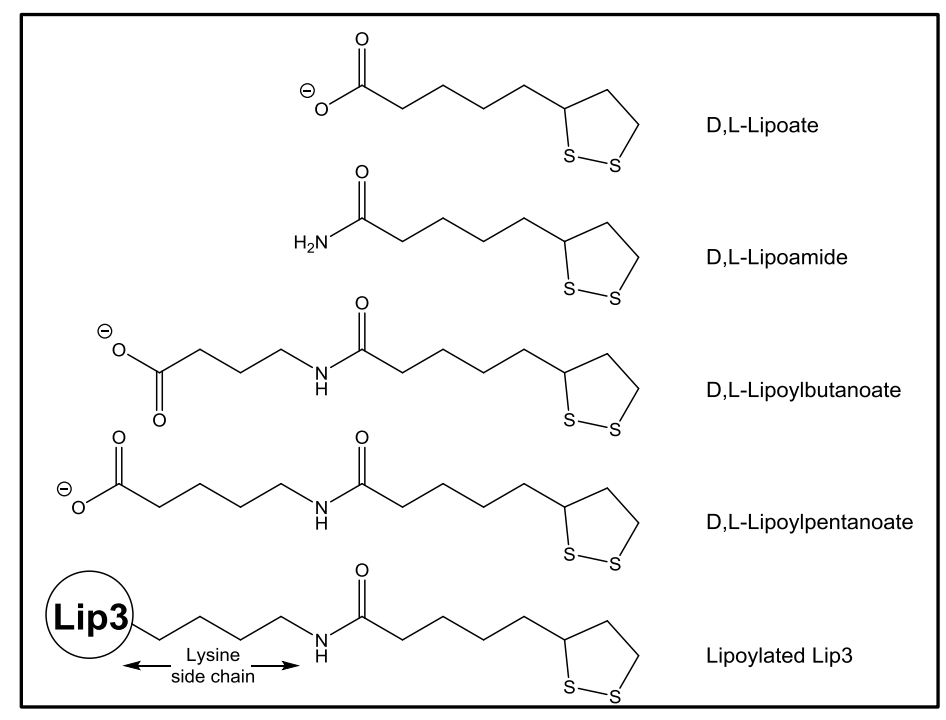

Figure 3.10 In vitro and native substrates of lipoamide dehydrogenases. D,L-lipoate and D,L-lipoamide reflect the most elementary artificial substrates and suffer from high $K_{\mathrm{M}}$ values and limited water solubility. D,Llipoylbutanoate and $\mathrm{D}, \mathrm{L}-$ lipoylpentanoate were synthesized and successfully used as substrates to overcome the mentioned problems showing increased catalytic proficiencies (Argyrou et al. 2003). EcLip3 is composed of the innermost lipoyl domain of $E c \mathrm{E} 2$ including lipoic acid

\subsubsection{Characterization of EcLip3 and its Reduction to the dihydro-form $\left(E c \mathrm{Lip3}_{2}\right)$}

Isolation and characterization of a single lipoyl domain has already been achieved, however, this isolated domain represented a hybrid between the first and third lipoyl domains of the E. coli E2 component (Ali and Guest 1990). In contrast, EcLip3 harbors the native EcE2 amino acid sequence (5.1). His $_{6}$-SUMO-EcLip3 could be purified in high amounts and removal of the fusion protein lead to the desired isolated domain. Since EcLip3 should serve as substrate for EcE3 the lipoylation status of the isolated domain after purification was essential since unlipoylated domains would compete with the active sites of the oxidoreductase and thus influence kinetic and spectroscopic parameters. The lipoylation status was checked by MALDI-TOF MS of tryptic fragments of EcLip3 (2.2.6.2). An MS approach without prior tryptic digestion to check the presence of the post-translational modification was described earlier (Seifert 2010; Wei et al. 2003). However, the application of the latter method is limited to the use of single isolated domains since a discrimination of two individual lipoylation sites is not possible. On the contrary, the generation and further analysis of tryptic fragments offers the potential for an future application on multidomain constructs, e.g. EcLip2-Lip3, or even the full-length $E c \mathrm{E} 2$ component. MALDI-TOF MS revealed that EcLip3 was fully lipoylated after purification 
ensuring homogeneity of the future substrate (Figure 3.11A). Therefore, an in vitro post-translational modification using lipoate-protein ligase A was not necessary.

Interaction of EcLip3 with $E c$ E3 was investigated by ITC using the same buffer conditions as used for the kinetic experiments (3.2.2). Binding between the two individual components was evident, however detected changes in enthalpy were rather small (Figure 5.20). Thus, a reliable determination of binding parameters was not possible. A comparable binding behavior was described for the interaction of the human lipoyl domain 2 ( $h \mathrm{Lip} 2)$ and the human pyruvate dehydrogenase ( $h \mathrm{E} 1)$ (Seifert 2010). It might be concluded that binding of lipoyl domains to other PDH components is always of a weak nature, however, this contrasts with earlier published results which showed binding constants in the lower micromolar range (Kato et al. 2008). The interaction of the motile parts of the PDH complexes are thus still a matter of controversial and were not further studied during this project. The affinity of reduced $E c \mathrm{Lip} 3\left(E c \mathrm{Lip}_{3} \mathrm{H}_{2}\right)$ to $E c \mathrm{E} 3$ was not investigated since $E c \mathrm{Lip}_{3} \mathrm{H}_{2}$ is able to serve as substrate (see below) und thus detected heat changes would reflect reduction of the flavin cofactor rather than binding events.

Reduction of a dithiolane moiety usually requires borohydride and low $\mathrm{pH}$ values (Argyrou et al. 2003), conditions which are not suitable for isolated proteins. Therefore, the transfer into dihydrolipoamide under physiological conditions is required and can be realized by the usage of TCEP (Wang et al. 2014). Reduction of $E c \mathrm{Lip} 3$ to $E c \mathrm{Lip} \mathrm{H}_{2}$ by TCEP leads to opening of the dithiolane moiety and an concomitant loss in absorbance at $333 \mathrm{~nm}$ (Figure 3.11B). According to far-UV CD spectra the secondary structure of $E c L$ Lip 3 is not affected by the reduction retaining its signature typical for $\beta$-turns (Perczel and Fasman 1992). The spectroscopic results are moreover in line with previously published NMR data of the aforementioned hybrid lipoyl domain (Green et al. 1995). 

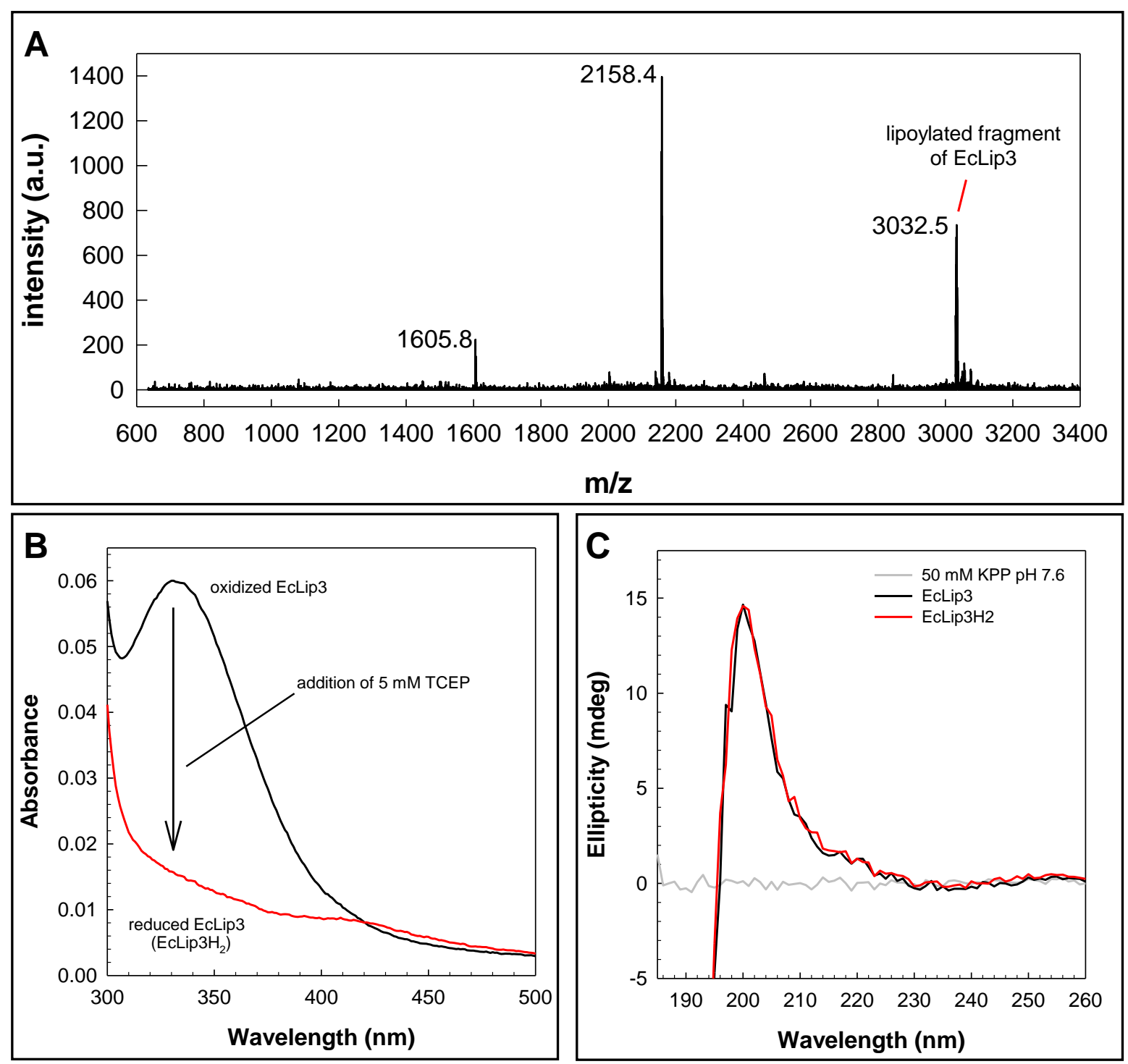

Figure 3.11 Evidence of the structural integrity of $E c \mathrm{Lip} 3$ and $E c \mathrm{Lip}_{3} \mathrm{H}_{2}$.

(A) MALDI-TOF MS analysis of tryptic EcLip3. Presence of the fragment with an $\mathrm{m} / z 3032.0$ shows that EcLip3 is fully lipoylated after purification (2.2.6.2). (B) Reduction of EcLip3 by TCEP to $E c \mathrm{Lip} 3 \mathrm{H}_{2}$ leads to decrease in absorbance at $333 \mathrm{~nm}$. (C) Far-UV-Vis CD spectra of EcLip3 and its dihydro-form $(0.1 \mathrm{mg} / \mathrm{mL}$ each). Reduction of the dithiolane moiety has no influence on the secondary structure. 


\subsection{2 $E c \mathrm{Lip} \mathrm{H}_{2}$ as native Substrate to track the reductive Half-reaction of $E c \mathrm{E3}$}

Since reduction of $E c \mathrm{Lip} 3$ to $E c \mathrm{Lip} 3 \mathrm{H}_{2}$ was successful, the reduced form should serve as native reducing agent for $E c E 3$ to track the reductive half-reaction using stopped-flow absorbance spectroscopy. Major emphasis was thereby devoted to the detection of the covalent FAD-C4acysteinyl adduct. Rapid reduction of $\mathrm{E}_{\text {ox }}$ by $E c \mathrm{Lip} \mathrm{H}_{2}$ under single turnover conditions transferred the oxidized form of the enzyme into its two-electron reduced state $\left(\mathrm{EH}_{2}\right)$. The absorbance increased at $390-440$ and $500-620 \mathrm{~nm}$, and decreased at 340-380 and 440-500 nm. The presence of the broad absorption band between 500-620 nm and the concomitant blue shift of the flavin peak reflects the charge-transfer situation between the anionic form of Cys50 and the flavin cofactor (Figure 3.12A). Titration of $E c \mathrm{E} 3$ with $>10$-fold molar excess of $\mathrm{Lip} 3 \mathrm{H}_{2}$ did not lead to $\mathrm{EH}_{4}$ formation indicating that the enzyme resided in the catalytically active $\mathrm{EH}_{2}$ state. This result is in disagreement with a previous study which demonstrated that dihydrolipoic acid is indeed able to overreduce the E. coli enzyme to the $\mathrm{EH}_{4}$ state under anaerobic conditions (Reed et al. 1960). The comparable reversible midpoint potential for dihydrolipoic acid was determined to be $-325 \mathrm{mV}$ at $\mathrm{pH} 7$ and $25{ }^{\circ} \mathrm{C}$ and is thus on par with that of NADH (Ke 1957; Unden and Bongaerts 1997). A comparable reduction behavior should be the consequence; however, reduction of $E c \mathrm{E} 3$ by the native domain seems to be more complex than with the low molecular weight substrates.

Formation of the charge-transfer band at $530 \mathrm{~nm}$ occurs monoexponentially with $\sim 180 \mathrm{~s}^{-1}$ (Figure 3.12C), which is approximately 3.5-fold faster compared to single turnover conditions during the oxidative half-reaction using NADH as reductant (3.1.2). The following decay proceeds with at least two exponentials and probably results in the multiple equilibria of the $\mathrm{EH}_{2}$ state as described in detail in 3.1.2 and 3.1.4. According to the absorbance trace at $380 \mathrm{~nm}$ there is no evidence for a significant accumulation of the covalent FAD-C4a-cysteinyl adduct, neither during Cys50-thiolate-formation nor during development of the different equilibrium species (Figure 3.12D). The decrease in this absorbance range absorbance is in fact based on the blue shift of the entire $\mathrm{EH}_{2}$ flavin signature.

Though a direct evidence for the cysteinyl-C4a adduct was not found, $E c \mathrm{Lip}_{3} \mathrm{H}_{2}$ was successfully used as a native reductant for $E c \mathrm{E} 3$ allowing the spectroscopic and kinetic investigation of the reductive half-reaction. Besides the usage during rapid reaction measurements, an application of EcLip3 $\mathrm{H}_{2}$ during classic steady-state kinetics is also imaginable. Purification yields and the fact that EcLip3 could be highly concentrated ( $>3 \mathrm{mM}$ ) would thus allow the determination of its catalytic proficiency. 

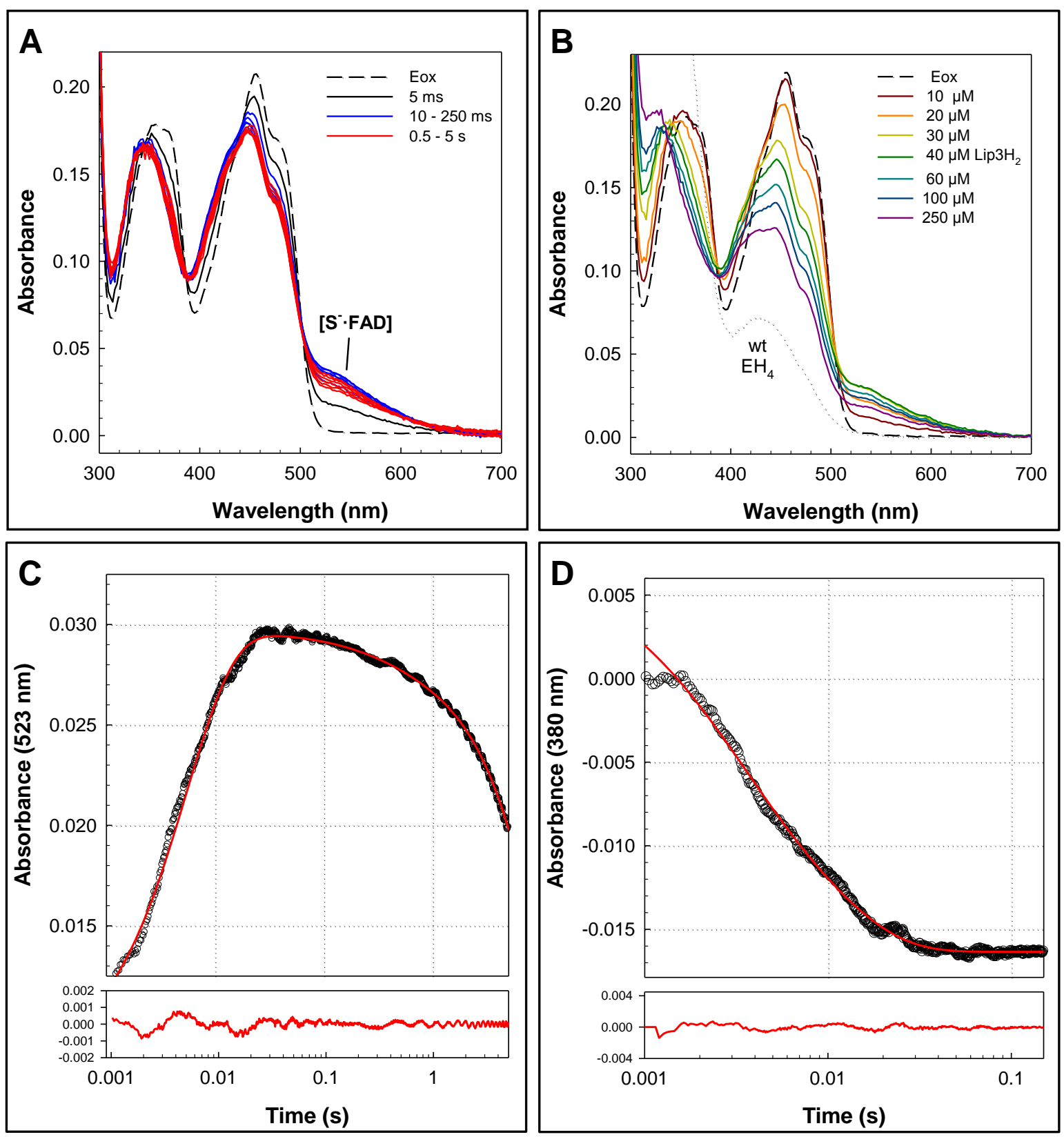

Figure 3.12 Reduction of $E c \mathrm{E} 3$ by $E c \mathrm{Lip} 3 \mathrm{H}_{2}$ under single turnover conditions.

(A) Stopped-flow absorbance traces after mixing $20 \mu \mathrm{M}$ EcE3 with $20 \mu \mathrm{M} \mathrm{EcLip3} \mathrm{H}_{2}$ at $4{ }^{\circ} \mathrm{C}$ and $\mathrm{pH}$ 7.6. The broad absorption band at $530 \mathrm{~nm}$ shows the presence of charge transfer between the Cys50 thiolate and the FAD. (B) Titration of EcE3 with $E c \mathrm{Lip}_{3} \mathrm{H}_{2}$. The dotted line reflects a typical wt-EH ${ }_{4}$ spectrum after reduction with excess of NADH. $>10$-fold molar excess of $E c \mathrm{LipH}_{2}$ did not lead to formation of $\mathrm{EH}_{4}$. (C and D) Absorbance traces at 523 and $523 \mathrm{~nm}$ and corresponding fits. The fits to a double exponential equation $\left(A=A 1 \cdot e^{-k 1 \cdot t}+A 2 \cdot e^{-k 2 \cdot t}\right)$, respectively. The points are the experimental data, and the solid lines are the fits to a triple and a double exponential equation $(\mathrm{A}=$ $\left.\mathrm{A} 1 \cdot \mathrm{e}^{-\mathrm{k} 1 \cdot \mathrm{t}}+\mathrm{A} 2 \cdot \mathrm{e}^{-\mathrm{k} 2 \cdot \mathrm{t}}\right)$, respectively. Note the logarithmic time-scales. 


\subsubsection{Evidence for the Transience of the Cysteinyl-C4a Adduct}

As described above, the covalent $\mathrm{C} 4 \mathrm{a}$-flavin adduct did not accumulate significantly neither in the whole reverse direction (3.1.2) nor during the isolated oxidative half-reaction (3.2.2). However, a spectroscopic evidence for an $\mathrm{NAD}^{+}$-induced adduct formation was achieved using a monothiol variant of $E c \mathrm{E} 3$ using conventional absorbance spectroscopy (3.1.5). According to the latter, the accumulation of the adduct in solution under equilibrium conditions is rather minute. The successful establishment of $E c \mathrm{Lip}_{3} \mathrm{H}_{2}$ as native reductant of $E c \mathrm{E} 3$ allows the spectroscopic characterization of the entire reaction cycle with the oxidative half-reaction as starting point. Therefore, EcE3 was transferred into the $\mathrm{EH}_{2}$ state by rapid reduction with $E c \mathrm{Lip} 3 \mathrm{H}_{2}$ followed by a second rapid mixing event with $\mathrm{NAD}^{+}$to complete the cycle using low-temperature sequential stopped-flow absorbance spectroscopy (Figure 3.13A). A comparable method was described earlier for the Mycobacterium tuberculosis lipoamide dehydrogenase, however, the $\mathrm{EH}_{2}$ pre-equilibrium was generated by titration with dithionite and not with a native substrate as described here (Argyrou et al. 2003).

The first mixing event generated the $\mathrm{EH}_{2}$ state of $E c \mathrm{E} 3$ indicated by its characteristic charge-transfer band at $530 \mathrm{~nm}$ (Figure 3.13B and C). After generation of the pre-equilibrium $(50 \mathrm{~ms}) \mathrm{NAD}^{+}$ (equimolar and 5-fold molar excess) was added in a second step leading to a loss in absorbance in the $530 \mathrm{~nm}$ range and concomitant increase at $340 \mathrm{~nm}$ within the dead-time of mixing. Though the first spectrum was recorded after $5 \mathrm{~ms}$ the generation of NADH $(340 \mathrm{~nm})$ and loss of the charge-transfer band indicate that a spectroscopic detection of the C4a-flavin adduct was not possible since the reaction already came to an end (Figure 3.4B and C). Hence, a spectroscopic detection of the $\mathrm{EH}_{2} \cdot \mathrm{NAD}^{+}$-complex was also not possible. The latter complex is characterized by a red-shifted (to $600 \mathrm{~nm}$ ) charge-transfer band which could be detected for the Mycobacterium tuberculosis and the pig heart enzymes (Argyrou et al. 2003; Wilkinson and Williams 1979). The increased overall reaction velocity of the E. coli enzyme compared to mentioned isoforms further complicates not only the detection of the $\mathrm{C} 4 \mathrm{a}-$-flavin adduct. Moreover, the determination of absolute $k_{\text {cat }}$ values in dependence of $\mathrm{NAD}^{+}$is not possible with the setup described above.

In conclusion, there is evidence that the covalent $\mathrm{C} 4 \mathrm{a}$-flavin adduct does not accumulate to a significant extent during catalysis. Its high instability and its transience were confirmed by monitoring the $E c \mathrm{E} 3$ reaction cycle from the forward $\left(\mathrm{EH}_{2}+\mathrm{NAD}^{+}\right)$and the reverse direction $\left(\mathrm{E}_{\mathrm{ox}}+\mathrm{NADH}\right)$. It was proposed that the instability of the intermediate is based on a faster decay compared to its relatively slow formation in both directions for the Mycobacterium tuberculosis enzyme (Argyrou et al. 2003). Thus, the transient nature of the covalent $\mathrm{C} 4 \mathrm{a}$-flavin adduct in lipoamide dehydrogenases seems to be a hallmark in this enzyme class. 

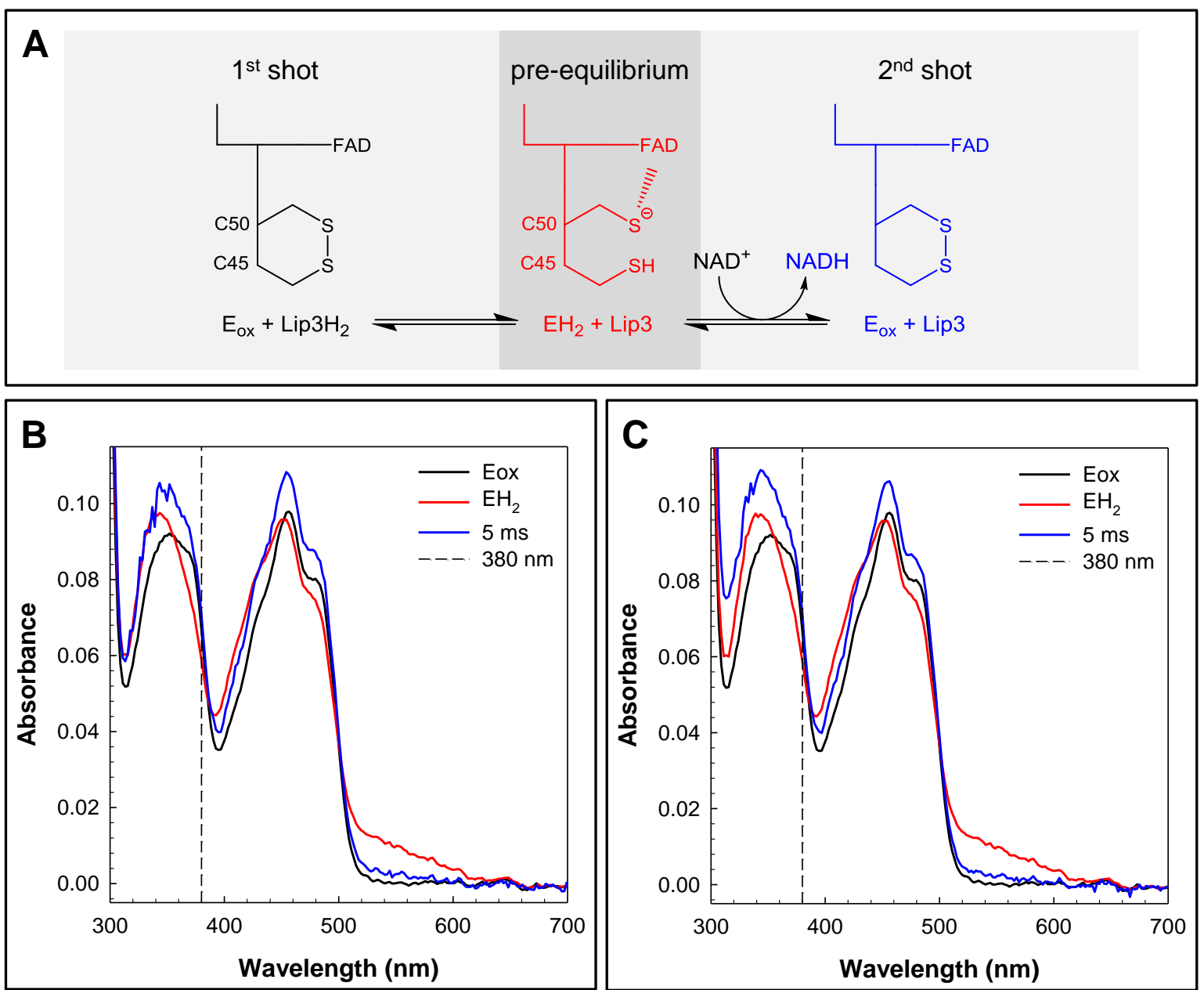

Figure 3.13 Sequential stopped-flow absorbance analysis of the $E c \mathrm{E} 3$ reduction by $E c \mathrm{Lip}_{3} \mathrm{H}_{2}$ and subsequent oxidation by $\mathrm{NAD}^{+}$at $4{ }^{\circ} \mathrm{C}$.

(A) Schematic of the sequential mixing experiment. First, $\mathrm{EcE} 3(40 \mu \mathrm{M})$ is mixed with $\mathrm{Lip}_{3} \mathrm{H}_{2}$ $(40 \mu \mathrm{M})$, forming the pre-equilibrium $\mathrm{EH}_{2}$ status of $E c \mathrm{E} 3$. Oxidation of $\mathrm{EH}_{2}$ is accomplished by the second mixing step $\left(50 \mathrm{~ms}\right.$ after the first step) with $\mathrm{NAD}^{+}$. (B) Oxidation of the pre-equilibrium with an equimolar amount $(20 \mu \mathrm{M})$ and $(\mathbf{C})$ with an 5-fold molar excess $(100 \mu \mathrm{M}) \mathrm{NAD}^{+}$. $\mathrm{E}_{\mathrm{ox}}$ reflects the oxidized spectrum - obtained by mixing solely with buffer, $\mathrm{EH}_{2}$ is the two-electron reduced state - obtained as described above - with subsequent mixing with buffer. $5 \mathrm{~ms}$ reflects the first possible spectrum recorded after mixing with $\mathrm{NAD}^{+}$. 


\subsection{Mechanistic Investigation of $E c \mathrm{E3}$}

The study thus far provided new insights in the catalytic function of lipoamide dehydrogenases. The findings were thereby mainly based on mutations targeting the redox active cystine moiety of the oxidoreductase. However, it was demonstrated that several other residues located around the active site harbor crucial functions during the catalytic cycle (Benen et al. 1991; Benen, Van Berkel, Dieteren, et al. 1992; Benen, Van Berkel, Veeger, et al. 1992; Maeda-Yorita et al. 1994). These and further residues were therefore investigated with the aforementioned methods to broaden the understanding of the reaction mechanism of $E c \mathrm{E} 3$.

It is described that the level to which lipoamide dehydrogenases are reduced beyond the $\mathrm{EH}_{2}$ state allows qualitative statements about the flavin redox potential (Maeda-Yorita et al. 1994). The latter fact will be used to expand the knowledge about the sensitivity of the flavin surrounding against structural alterations. Since the reaction cycle can be subdivided into a reductive and an oxidative halfreaction the results will be presented following the order of this subdivision starting with the reductive half-reaction (3.3.1). The oxidative half-reaction will be investigated in 3.3.2. An overview of all studied residues and their occurrence in lipoamide dehydrogenases from several organisms is given in the appendix (Figure 5.4).

\subsubsection{Characterization of the Reductive Half-Reaction using selected Active Site Variants}

In the reductive half-reaction, the substrate dihydrolipoamide binds to the $\mathrm{E}_{\mathrm{ox}}$ form of $E c \mathrm{E} 3$, and Cys45 forms a mixed disulfide with the substrate. Transfer of electrons leads to formation of the Cys50- thiolate and thus the two-electron reduced state $\left(\mathrm{EH}_{2}\right)(1.5)$. This reaction is general-acid-base catalyzed by the essential and conserved catalytic diad located at the C-terminus of the adjacent monomer, namely His455 and Glu450 (Figure 3.14). Release of lipoamide completes the oxidative half-reaction. A further investigation of the Glu-His pair with respect to their individual roles during catalysis is therefore interesting. In addition, a highly conserved tyrosine residue is also located at the putative lipoamide entry site (Figure 3.14). In order to elucidate whether Tyr19 also harbors an essential catalytic function as it has been described for the diad (Benen et al. 1991; Benen, Van Berkel, Dieteren, et al. 1992; Kim and Patel 1992a) it was also targeted for the mechanistic investigation. 


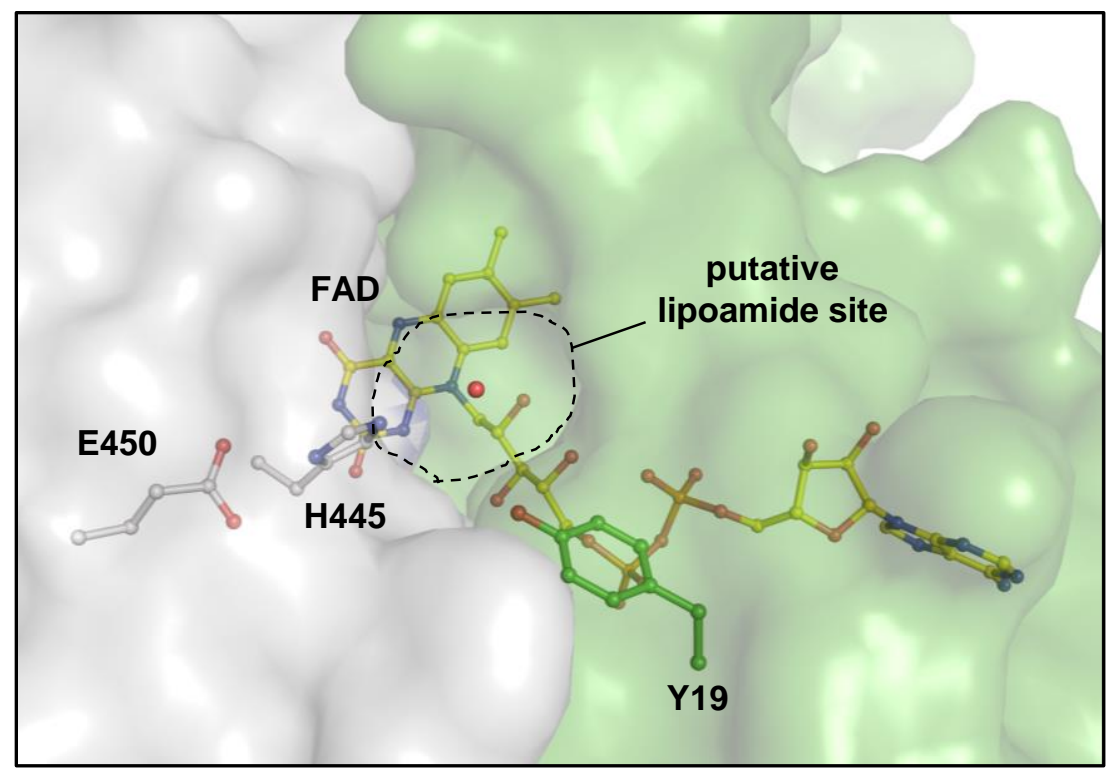

Figure $3.14 \quad$ Putative position of the lipoamide binding site.

The residues studied involved in the reductive half-reaction are located at the si face of the FAD and comprise the highly conserved catalytic diad; His445 and Glu450; and Tyr19. The putative entry site for the lipoamide cofactor is located at the dimer interface. The displayed water molecule

The visible absorption spectra of EcE3Y19F, H445A and E450Q are very similar to that of the wild type enzyme (5.2). This indicates that the mutations did not influence the surrounding of the flavin to a significant amount. Mutations at the site of the catalytic diad, however, had severe impact on the catalytic activity (Table 3.1). EcE3H445A showed below $0.1 \%$ residual activity under standardized assay conditions (5.4), but it should be noted that the actual activity might even be lower. When NADH served as reductant it cannot be excluded that reducing equivalents are transferred to other electron acceptors than lipoamide during diaphorase reactions. Hence, it is most likely that molecular oxygen oxidizes the reduced flavin intermediate leading to NADH consumption (5.4). The impact of mutation of Glu450 was not that drastic compared to its bonding partner showing $4 \%$ residual activity. According to far-UV CD spectroscopy mutation of Tyr19 to phenylalanine did not affect the secondary structure of the enzyme though it is located directly at the dimer interface (5.6). In addition the mutation only slightly altered the rate of substrate turnover (Table 3.1). 


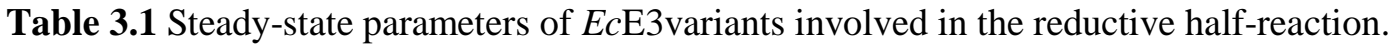
Parameters were calculated from data of the reverse steady-state assay (2.2.4.1). Plots of lipoamide dependence for individual variants are shown in the appendix (5.4).

\begin{tabular}{ccc} 
Variant & $\mathbf{A}_{\text {Spec }}(\mathbf{U} / \mathbf{m g})$ & $\boldsymbol{K}_{\mathbf{D}}\left(\mathbf{N A D}^{+}\right)(\boldsymbol{\mu M})$ \\
\hline wt & $72.9 \pm 4.6$ & $400^{*}$ \\
Y19F & $70.7 \pm 3.3$ & n.d. \\
H445A & $0.06 \pm 0.01$ & n.d. \\
E450Q & $2.9 \pm 0.1$ & n.d. \\
\hline
\end{tabular}

*(Wilkinson and Williams 1981)

\subsubsection{Histidine445 and Glutamate450 - The Role of the Catalytic Diad during Catalysis}

As the steady-state assays demonstrated mutation of the catalytic diad severely slowed down the reaction rates of the respective enzyme. Therefore, they were likely candidates that are potentially able to stabilize the covalent $\mathrm{C} 4 \mathrm{a}$-cysteinyl adduct. An investigation using rapid reactions measurements was hence obligate. Reduction of $E c \mathrm{E} 3 \mathrm{H} 445 \mathrm{~A}$ with $\mathrm{NADH}$ leads to spectroscopic properties which differ from that of the wild type enzyme (Figure 3.15A and B). Though a decrease in absorbance of the flavin is observable under single turnover conditions the typical blue-shift of the latter is way less pronounced. Moreover, there is no significant rise in absorbance in the $523 \mathrm{~nm}$ range indicating the Cys50-thiolate charge transfer complex with the FAD. However, the highly transient $\mathrm{NAD}^{+}-\mathrm{FADH}_{2}$ complex is significantly stabilized in this variant indicated by the broad charge transfer band in the longer wavelength region. Reduction with 5-fold excess of NADH lead to a complete flavin reduction (Figure 3.15B), but also in this case there is no spectroscopic evidence for reduction of the disulfide. On the contrary, the $\mathrm{NAD}^{+}-\mathrm{FADH}_{2}$ complex accumulates to a way higher extent according to the broad absorption band between 600 and $700 \mathrm{~nm}$. Reduction of $E c E 3 E 450 \mathrm{Q}$ with NADH leads to comparable results with respect to accumulation of the nucleotide-flavin complex. However, the presence of the charge transfer bands at $523 \mathrm{~nm}$ suggests that the intramolecular electron transfer to the disulfide is still possible albeit retarded when compared with the wild type enzyme (Figure 3.15C). During all experiments using NADH as reductant no increase in the 380-nm absorbance range was detected that would indicate a stabilization of the flavin adduct. 

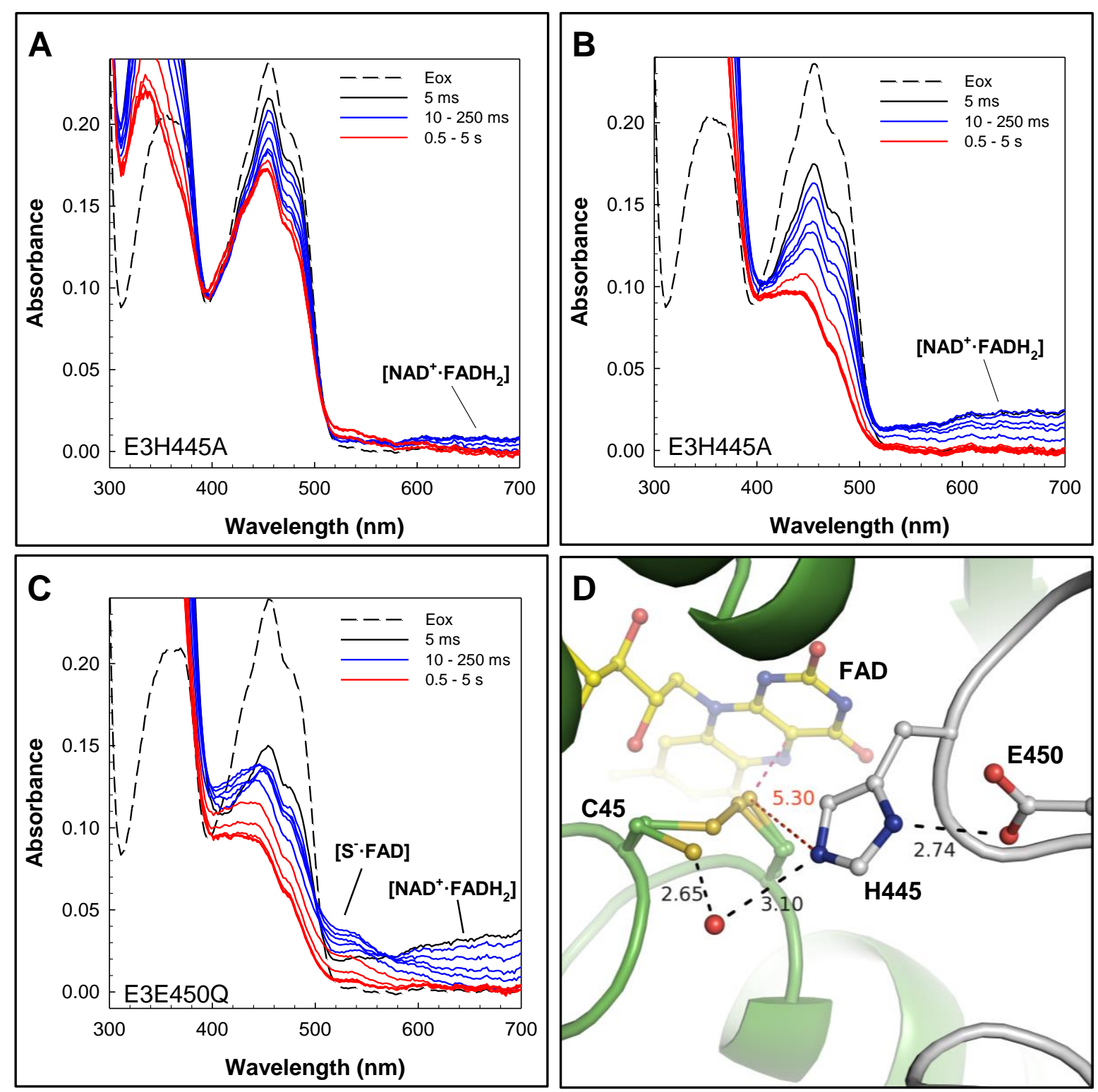

Figure 3.15 General characterization of the $E c E 3$ catalytic diad - His 445 and Glu450.

Stopped-flow absorbance traces of EcE3 variants upon reduction with NADH (A - C). $20 \mu \mathrm{M}$ $E c$ E3H445A were mixed with $20 \mu \mathrm{M}$ (panel A) or with $100 \mu \mathrm{M}$ NADH (B) at $4{ }^{\circ} \mathrm{C}$ and $\mathrm{pH}$ 7.6. (C) Reduction of $E c \mathrm{E} 3 \mathrm{E} 450 \mathrm{Q}$ with $100 \mu \mathrm{M}$ NADH. The dashed line represents the oxidized form of the respective enzyme $\left(E_{o x}\right)$ derived from a single mixing event with buffer. The solid spectra were recorded using a diode-array detector after mixing at the concentrations indicated. (D) Hydrogen bond network between the catalytic diad and the redox active cystine. N3 of His445 is too far away for a direct stabilization of the Cys50-thiolate (see 3.1.4). A putative interaction with a Cys45thiolate is likely mediated via a water molecule (red sphere). Glu450 ensures the correct orientation of the imidazole moiety of His 445 (distances in $\AA$ ).

The reduction of the mutated enzymes with NADH allowed the distinction of separate reactions steps and a subsequent comparison to the wild type. In both cases, the hydride transfer from NADH to FAD is fast and leads to a pronounced accumulation of the $\mathrm{NAD}^{+}-\mathrm{FADH}_{2}$ complex in comparison to the wild type. This indicates, that the transfer of reducing equivalents from the reduced flavin to the redox active disulfide is significantly slowed down (E450Q) or even impaired (H445A). The H445A mutant 
was not capable to stabilize the Cys50-thiolate charge transfer neither under single turnover conditions, nor in the presence of a five-fold molar excess of NADH. The small increase in the $523 \mathrm{~nm}$ region which can be observed in Figure 3.15A is most likely due to the offset correction of the spectra rather than the formation of a true thiolate species. The absence of a hypsochromic shift of the flavin peak underpins the assumption that no reduction of the disulfide occurs. Further, the almost complete loss of catalytic activity of $E c \mathrm{E} 3 \mathrm{H} 445 \mathrm{~A}$ enhances the latter assumption. These results are in agreement with previous studies on the E3 from A. vinelandii which showed that the conserved histidine acts as catalyst being in involved in (de)protonation of the interchange thiol (Cys45) and the substrate dihydrolipoamide, a step which is essential for an opening of the disulfide (Benen et al. 1991; Benen, Van Berkel, Dieteren, et al. 1992). Structural data suggest that a direct interaction of His445 with the Cys50-thiolate is not very likely (Figure 3.15D). Stabilization of the latter thiolate was discussed in a previous chapter (3.1.4). However, it was suggested that the interchange thiol can also be present in its anionic form since the $\mathrm{EH}_{2}$ state of $E c \mathrm{E} 3$ is actually a mixture of distinct spectroscopic species (Benen, Van Berkel, Dieteren, et al. 1992; Wilkinson and Williams 1979). It was suggested that the interchange thiolate can be stabilized by an interaction with an hydronium ion (Benen et al. 1991) due to the easy accessibility of the latter residue to the solvent. In fact, the EcE3 ground state structure revealed the presence of such water molecule which is coordinated by both His 445 and the open conformer of Cys45 (Figure 3.15D).

When combining the spectroscopic and structural data the catalytic role of Glu450 during catalysis can be proposed. Glu450 ensures the correct orientation of the imidazole moiety of His445 towards Cys45 and the putative entry site for dihydrolipoamide (Figure 3.15D). A substitution of this residue with glutamine consequently leads to a weakening or a loss of the hydrogen bond with the imidazole of His445 which most likely alters the orientation of the histidine relative to the interchange thiol. Further, this might also influence the $\mathrm{pKa}$ of the histidine, an aspect which has been discussed elsewhere (Benen et al. 1991). In contrast to His445, Glu450 is not essential for catalysis, but it is indicative that the carboxylic group of Glu450 functions as both as mediator for the $\mathrm{pKa}$ (Benen et al. 1991) and as structural element positioning the imidazole N3 towards the redox active disulfide.

Though the transfer of the reducing equivalents is slowed down $(\sim 5$-fold with respect to thiolate formation) in $E c \mathrm{E} 3 \mathrm{E} 450 \mathrm{Q}$ there has not been evidence for the accumulation of the covalent flavincysteinyl intermediate. It was suggested that the decay of the $\mathrm{C} 4 \mathrm{a}$ adduct might be faster than its formation in either direction making an spectroscopic detection tedious work (Argyrou et al. 2002; Benen, Van Berkel, Dieteren, et al. 1992). Apparently, this also holds true for the E. coli enzyme. In the case of the His445 mutant the absence might be even more coherent assuming that an opening of the disulfide is entirely impaired in this variant. 


\subsubsection{Tyrosine19 acts as structural Element rather than as a catalytic Residue}

Mutation of Tyr19 to a phenylalanine did not influence the specific activity of the enzyme to high extent in the standard assay (5.4). In addition, rapid reaction experiments confirmed that the latter mutation also did not have an effect on the spectral properties during both reduction with NADH and $E c \mathrm{Lip}_{3} \mathrm{H}_{2}$ (Figure 3.16). Formation of the Cys50-thiolate flavin complex can be observed during both reduction processes with similar rates, respectively, when comparing to the wild type enzyme. In addition, the highly transient $\mathrm{NAD}^{+}-\mathrm{FADH}_{2}$ complex does not accumulate during reduction with $\mathrm{NADH}$ indicating that the transfer of reducing equivalents to the redox active cystine is not influenced.
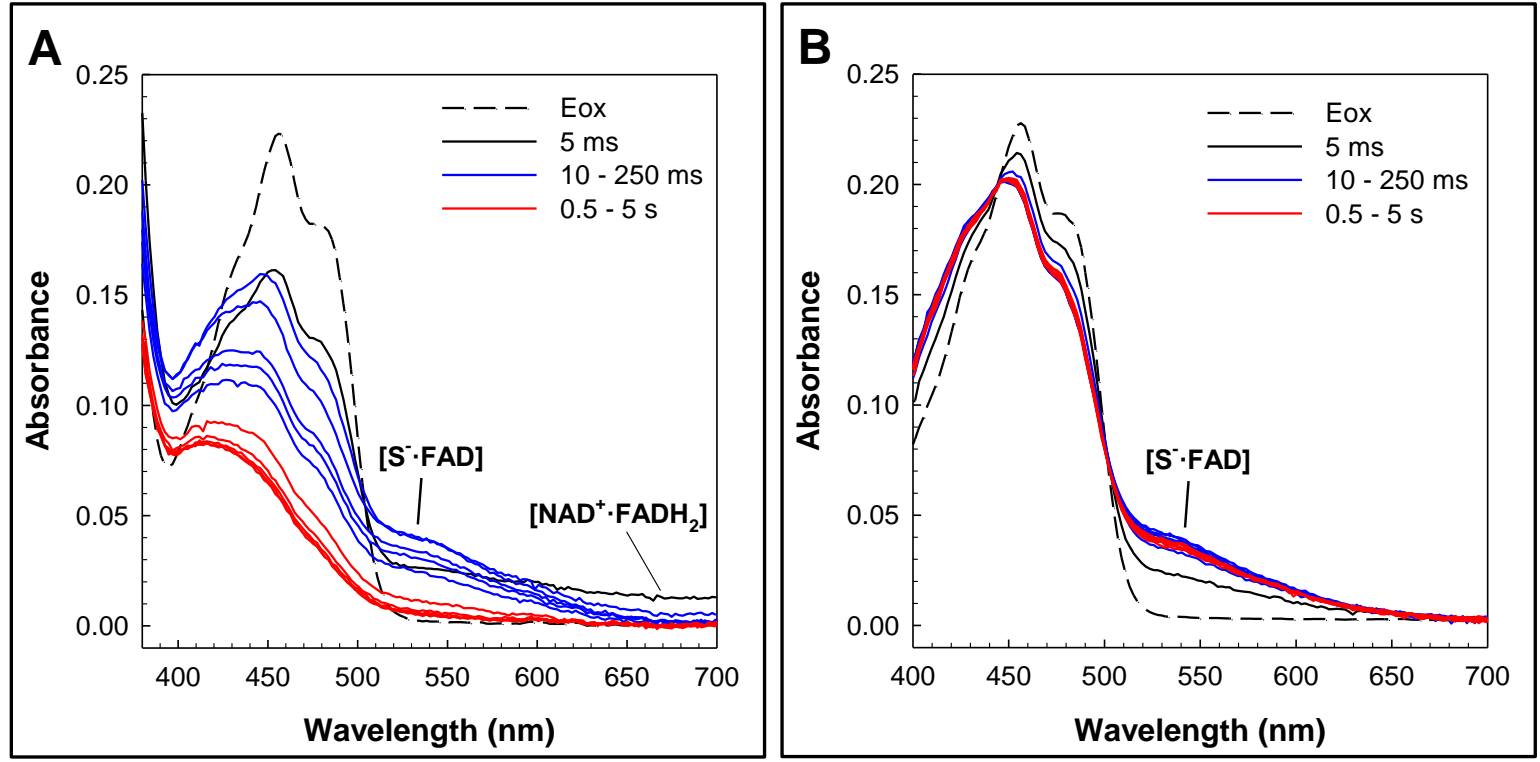

Figure 3.16 Stopped-flow absorbance traces of $E c \mathrm{E} 3 \mathrm{Y} 19 \mathrm{~F}$.

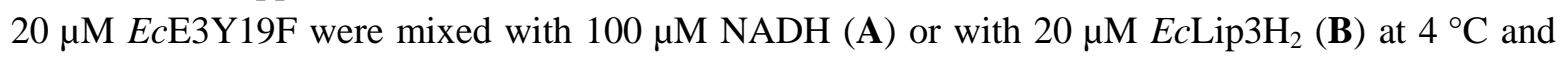
$\mathrm{pH}$ 7.6. The dashed lines represent the oxidized form of $E c \mathrm{E} 3 \mathrm{Y} 19 \mathrm{~F}\left(\mathrm{E}_{\mathrm{ox}}\right)$ derived from a single mixing event with buffer. The solid spectra were recorded using a diode-array detector after mixing at the concentrations indicated.

According to the results obtained it is most likely that Tyr19 does not contribute to catalysis in $E c \mathrm{E} 3$ since removal of the hydroxyl function did not alter the catalytic abilities of the enzyme to a significant extent. However, these results are contradicting with studies on the corresponding residue in the A. vinelandii E3 (Tyr16 according to A. vinelandii numbering). For this enzyme variant no activity was observed during steady-state assays using NADH as reductant (Benen, Van Berkel, Veeger, et al. 1992). Moreover, the mutated enzyme was easily overreduced by NADH while lacking the thiolate-flavin complex upon reduction demonstrating that the reducing equivalents mainly resided on the flavin rather than being transferred to the disulfide (Benen, Van Berkel, Veeger, et al. 1992). 
These spectral properties are similar to that of the $E c \mathrm{E} 3$ variants which were used to study the oxidative half-reaction (3.3.2). Hence, the tyrosine raised the redox potential of the flavin in the A. vinelandii enzyme. This elevation was not observed in the E. coli mutant where a 5-fold molar excess of NADH was required to overreduce the enzyme to the dead-end $\mathrm{EH}_{4}$ state - the same amount which was also required for $E c \mathrm{E} 3 \mathrm{wt}$.

In the A. vinelandii enzyme Tyr16 hydrogen bonds with His470 of the C-terminal part of the other subunit (Mattevi et al. 1991). It was speculated that this interaction stabilizes the homodimer thus contributing to optimal catalysis and protection against inhibition/overreduction by NADH (Benen, Van Berkel, Veeger, et al. 1992). However, it remained unclear how this interaction could influence the flavin redox potential since this interaction is far away from the active site. It was therefore speculated that the dimer interaction itself modulates the redox properties, but a clear proof was not given (Benen, Van Berkel, Veeger, et al. 1992). In EcE3, Tyr19 does not contribute to the subunit interaction via hydrogen bonding to a residue of the other subunit. Thus, a cross-talk between the two monomers assisted by Tyr19 is not likely. Moreover, the aforementioned histidine of the A. vinelandii enzyme is not present in the EcE3 (Figure 5.4). Hence, the results obtained for EcE3Y19F, which reflects a wild type behavior, are not unexpected in the light of catalysis potentially regulated by monomer-monomer interaction. That the flavin redox potential indeed can be regulated by mutation of a another residue present at the dimer interface (Glu354) will be shown and discussed in a following chapter (3.5).

\subsubsection{Characterization of the Oxidative Half-reaction using selected Active Site Variants}

In the oxidative half-reaction, $\mathrm{NAD}^{+}$binds to the two-electron reduced state of the enzyme $\left(\mathrm{EH}_{2}\right)$. This induces an internal electron transfer from the redox active disulfide towards the flavin cofactor yielding a transiently formed $\mathrm{FADH}_{2}$ intermediate. The flavin is immediately reoxidized by $\mathrm{NAD}^{+}$ generating NADH and the oxidized form of the enzyme $\left(\mathrm{E}_{\mathrm{ox}}\right)$. Release of NADH completes the reductive half-reaction.

Replacements of residues at the site of the catalytic diad significantly decreased or even depleted the rate of substrate turnover, but an accumulation of the flavin-C4a-cysteinyl adduct did not occur (3.3.1.1). Therefore, the investigation was expanded to residues at the $r e$ face of the flavin which are thought to assist (de)protonation of the N5 atom of the flavin cofactor during the oxidative halfreaction (Maeda-Yorita et al. 1994) and therefore site directed mutagenesis of the residues involved might lead to $E c \mathrm{E} 3$ variants that accumulate the covalent intermediate. 
The ammonium function of Lys54 is located close to the isoalloxazine ring and makes a weak polar interaction with $\mathrm{N} 5$ (Figure 3.17). The previously solved structure of $E c E 3$ (PDB: 4JDR) demonstrated that the interaction of Lys54 with N5 is further mediated by a water molecule (Figure 1.4) (Chandrasekhar et al. 2013). Difference map peaks ( $\mathrm{mFo}-\mathrm{DFc}$ at 3 sigma level) were visible in the same region for the structure presented here, but the peaks were too weak to allow an appropriate model building. However, the presence of a water molecule at the mentioned position is not unlikely. Besides the interaction with the cofactor, the lysine residue forms an ion pair with Glu189 positioning the positive end of this salt bridge towards the flavin (Figure 3.17). These two residues are not only conserved in E3s from different organisms (Figure 5.4), but also in all homologous enzymes of the pyridine nucleotide-disulfide oxidoreductase family. It has been suggested and later on demonstrated that the positive charge of the lysine plays a crucial role in mediating the redox potential of the flavin in both glutathione reductase as well in $E c \mathrm{E} 3$ thus contributing to optimal catalysis (Maeda-Yorita et al. 1994; Schulz and Pai 1983). Both residues were therefore mutated to glutamines, respectively, which lead to a disruption of the ion pair interaction but still allowed hydrogen bonding interactions.

$\operatorname{Arg} 386$ of the adjacent monomer is located at the dimer interface and the distance of its guanidinium group is $\sim 7 \AA$ to the $\varepsilon$-amino group of Lys54 (Figure 3.17). The corresponding residue (Arg382) in E. coli mercuric ion reductase is thought to participate in a proton transfer reaction during catalysis by a direct interaction with the catalytic lysine (Susan Miller, University of California, personal communication). Hence, this residue was also targeted for the mechanistic investigation aiming to elucidate whether a perturbation of its proposed function yields in stabilization of the covalent flavin adduct.

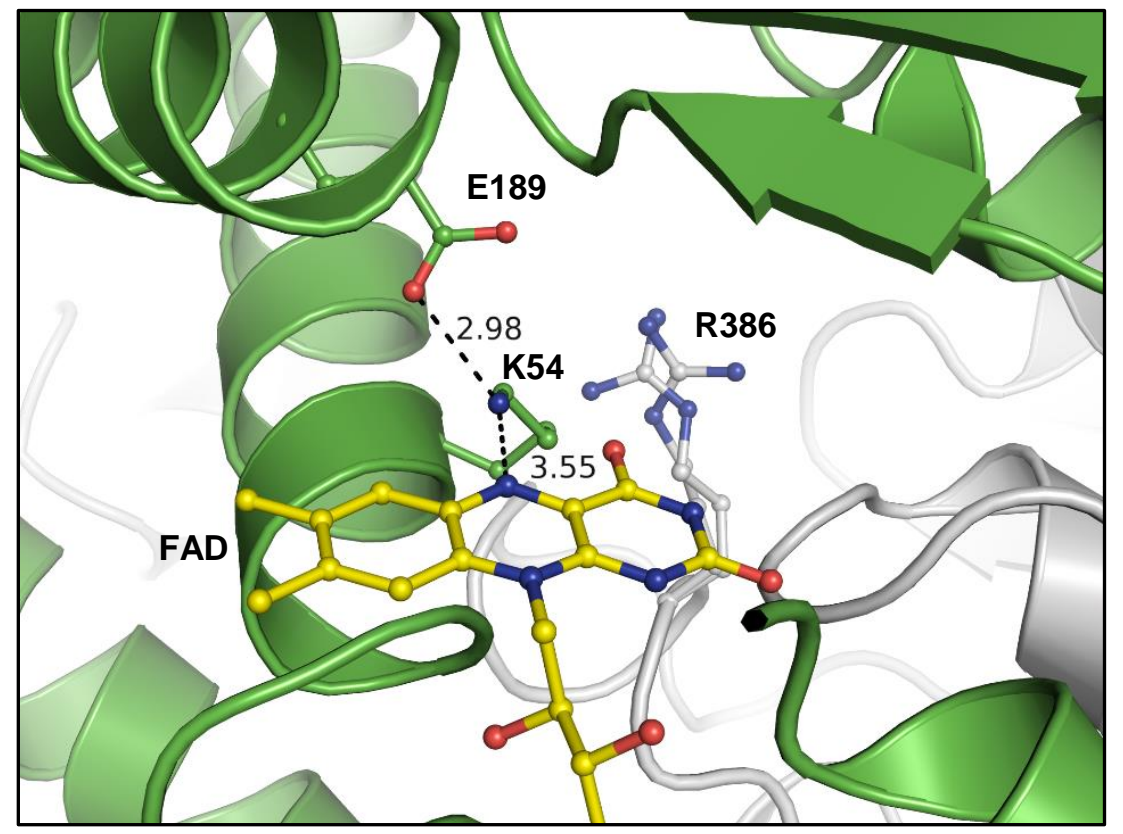

Figure 3.17 Overview of the $r e$ face of the FAD.

Lys54 makes a polar interaction with N5 (distances in $\AA$ ). The residue is thereby positioned by Glu189. In the previously solved structure of $E c E 3$ (PDB: 4JDR) the interaction of Lys54 with N5 is further mediated by a water molecule (Chandrasekhar et al. 2013). Arg386 of the adjacent monomer (grey) is located at the dimer interface. For the putative role of this residue see text. 
The visible absorption spectra of $E c E 3 K 54 \mathrm{Q}$ and E189Q harbor minor changes when compared to the wild type (5.2). In both cases the main flavin band is slightly blue-shifted and the shoulder at $487 \mathrm{~nm}$ is less pronounced. This might be indicative for a more hydrophilic environment around the flavin (Harbury et al. 1959). In contrast, the UV-Vis spectrum of $E c E 3 R 386 \mathrm{~A}$ was almost identical to that of the wild type enzyme. Mutations near the flavin N5 site had severe impact on the catalytic activity (Table 3.2). EcE3K54Q only showed $\sim 0.5 \%$ residual activity under standardized assay conditions. Moreover, replacement of the negatively charged Glu189 lead to a further reduction of the enzymatic activity $(<0.2 \%)$. However, the latter activity also tends to be defective according to aforementioned limitations of the reverse assay (3.3.1). Mutation of Arg386 to alanine did not abolish the catalytic function of the enzyme. The variant showed $\sim 22 \%$ residual activity indicating that an involvement of the latter residue into a proton transfer reaction is questionable, at least with respect to its steady state activity.

Table 3.2 Steady-state parameters of $E c E 3$ variants involved in the oxidative half-reaction.

Parameters were calculated from data of the reverse steady-state assay (2.2.4.1). Plots of lipoamide dependence for individual variants are shown in the appendix (5.4).

\begin{tabular}{ccc} 
Variant & $\mathbf{A}_{\text {Spec }}(\mathbf{U} / \mathbf{m g})$ & $\boldsymbol{K}_{\mathbf{D}}\left(\mathbf{N A D}^{+}\right)(\boldsymbol{\mu M})$ \\
\hline wt & $72.9 \pm 4.6$ & $400 *$ \\
K54Q & $0.33 \pm 0.01$ & $42.0 \pm 1.8$ \\
E189Q & $0.14 \pm 0.01$ & n.d. \\
R386A & $16.3 \pm 0.4$ & $155 \pm 16$ \\
\hline
\end{tabular}

* (Wilkinson and Williams 1981)

Reduction of $E c E 3 K 54 \mathrm{Q}$ with NADH under single turnover conditions in a stopped-flow apparatus demonstrated a strong accumulation of the $\mathrm{NAD}^{+}-\mathrm{FADH}_{2}$ charge transfer band between $600-700 \mathrm{~nm}$. Moreover, almost a complete reduction of the flavin could be observed thereby lacking the characteristic blue shift and the Cys50-thiolate FAD charge transfer (Figure 3.18A). $\mathrm{NAD}^{+}-\mathrm{FADH}_{2}$ complex formation occurs monoexponentially with $\sim 103 \mathrm{~s}^{-1}$ and results in stabilization of the latter complex for $\sim 300 \mathrm{~ms}$ (Figure 3.18B) prior to its decay. NADH titration revealed that in fact one equivalent $\mathrm{NADH}$ is sufficient to reduce one equivalent of $E c \mathrm{E} 3 \mathrm{~K} 54 \mathrm{Q}$ (Figure 3.18C). Hence, internal transfer of reducing equivalents from the flavin site towards the redox active disulfide seems to be impaired. The pronounced nucleotide-flavin charge transfer indicates a strong binding of $\mathrm{NAD}^{+}$to the enzyme. This tightened binding was confirmed by $\mathrm{NAD}^{+}$-titrations giving an apparent $K_{\mathrm{D}}$ of $42 \mu \mathrm{M}$. 
The binding constant for $\mathrm{NAD}^{+}$is thus almost 10 -fold lower to that observed for the wild type enzyme (Wilkinson and Williams 1981).

Interestingly, the same qualitative results were observed for $E c \mathrm{E} 3 \mathrm{R} 386 \mathrm{~A}$; for this variant the apparent $K_{\mathrm{D}}$ was $\sim 3$-fold higher than for $E c \mathrm{E} 3 \mathrm{~K} 54 \mathrm{Q}$ (Figure 5.5). In addition, $E c \mathrm{E} 3 \mathrm{R} 386 \mathrm{~A}$ was able to form the Cys50-thiolate FAD charge transfer when EcLip3H2 served as reductant (Figure 5.5D). 

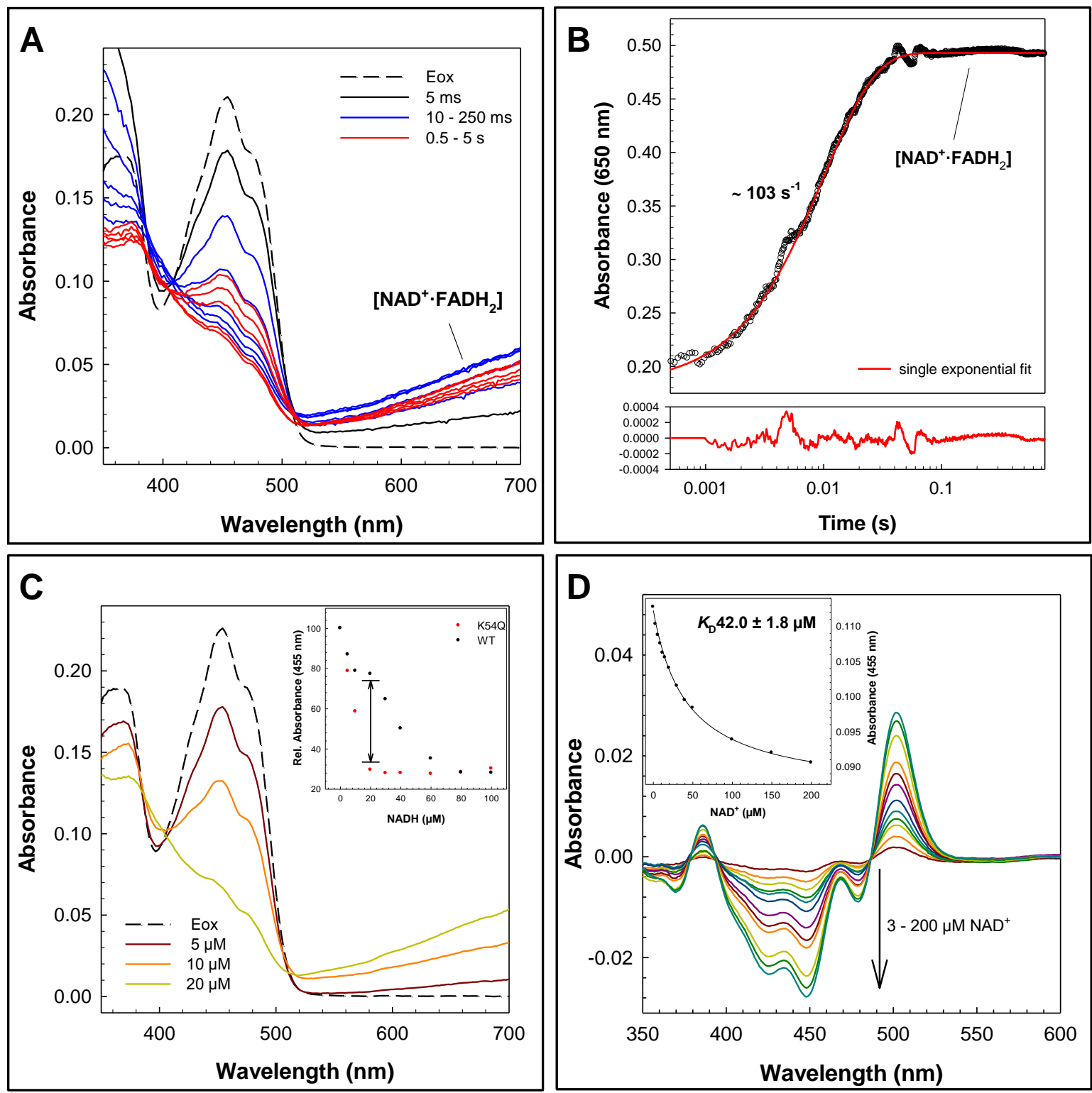

Figure 3.18 Spectral properties of EcE3K54Q.

(A) Stopped-flow absorbance traces after mixing of $20 \mu \mathrm{M} \mathrm{EcE3K54Q} \mathrm{with} 20 \mu \mathrm{M}$ NADH at $4{ }^{\circ} \mathrm{C}$ and $\mathrm{pH}$ 7.6. The dashed line represents the oxidized form of the respective enzyme $\left(\mathrm{E}_{\mathrm{ox}}\right)$ derived from a single mixing event with buffer. The solid spectra were recorded using a diode-array detector after mixing. (B) Stopped-flow absorbance trace at $650 \mathrm{~nm}$ under same conditions. The points are the experimental data, and the solid line is the fit to a single exponential equation $\left(\mathrm{A}=\mathrm{A} 1 \cdot \mathrm{e}^{-\mathrm{kl} \cdot \mathrm{t}}\right)$. Note the logarithmic time-scale. $\mathrm{NAD}^{+}-\mathrm{FADH}_{2}$ complex formation occurs with $\sim 103 \mathrm{~s}^{-1}$ and runs into an transient equilibrium prior to its decay. (C) Absorbance traces derived from a stopped-flow $\mathrm{NADH}$ titration at $4{ }^{\circ} \mathrm{C}$ and $\mathrm{pH}$ 7.6. Complete reduction of the flavin can be achieved using an equimolar amount of NADH. The inset shows the relative absorbances at $455 \mathrm{~nm}$ plotted against the respective $\mathrm{NADH}$ concentration. Internal transfer of reducing equivalents to the disulfide is therefore most unlikely. (D) Lower temperature $\mathrm{NAD}^{+}$titration (difference spectra) at $10{ }^{\circ} \mathrm{C}$ and $\mathrm{pH}$ 7.6. The inset shows a hyperbolic fit for the decrease in absorbance at $455 \mathrm{~nm}$ giving an apparent binding constant of $\sim 42 \mu \mathrm{M}$ for the nucleotide. 
A 5-fold molar excess of NADH was not sufficient to induce EH4 formation thus, $E c$ E3E189Q did not suffer from severe overreduction by NADH (Figure 3.19A and Figure 5.6A). However, the more pronounced accumulation of the $\mathrm{NAD}^{+}-\mathrm{FADH}_{2}$ complex and the absence of the Cys50-thiolate FAD charge transfer were also present in this variant. $\mathrm{NAD}^{+}$titrations did not indicate a lowered binding constant for the nucleotide since no significant quenching of the flavin was observed (Figure 5.6B). Further, E189Q is also not capable to stabilize the Cys50-thiolate FAD charge transfer to a significant amount when using $E c \mathrm{Lip} 3 \mathrm{H}_{2}$ as reductant (Figure 3.19B) and reoxidation of the cofactor occurs remarkably faster than in the wild type enzyme.

In summary, the alterations at residues near the flavin N5 influenced the spectroscopic and catalytic behavior of all three mutated enzymes studied. However, during all experiments using NADH or $E c \mathrm{Lip}_{3} \mathrm{H}_{2}$ as reductant no increase in the $380-\mathrm{nm}$ absorbance range was detected that would indicate a stabilization of the covalent flavin adduct.
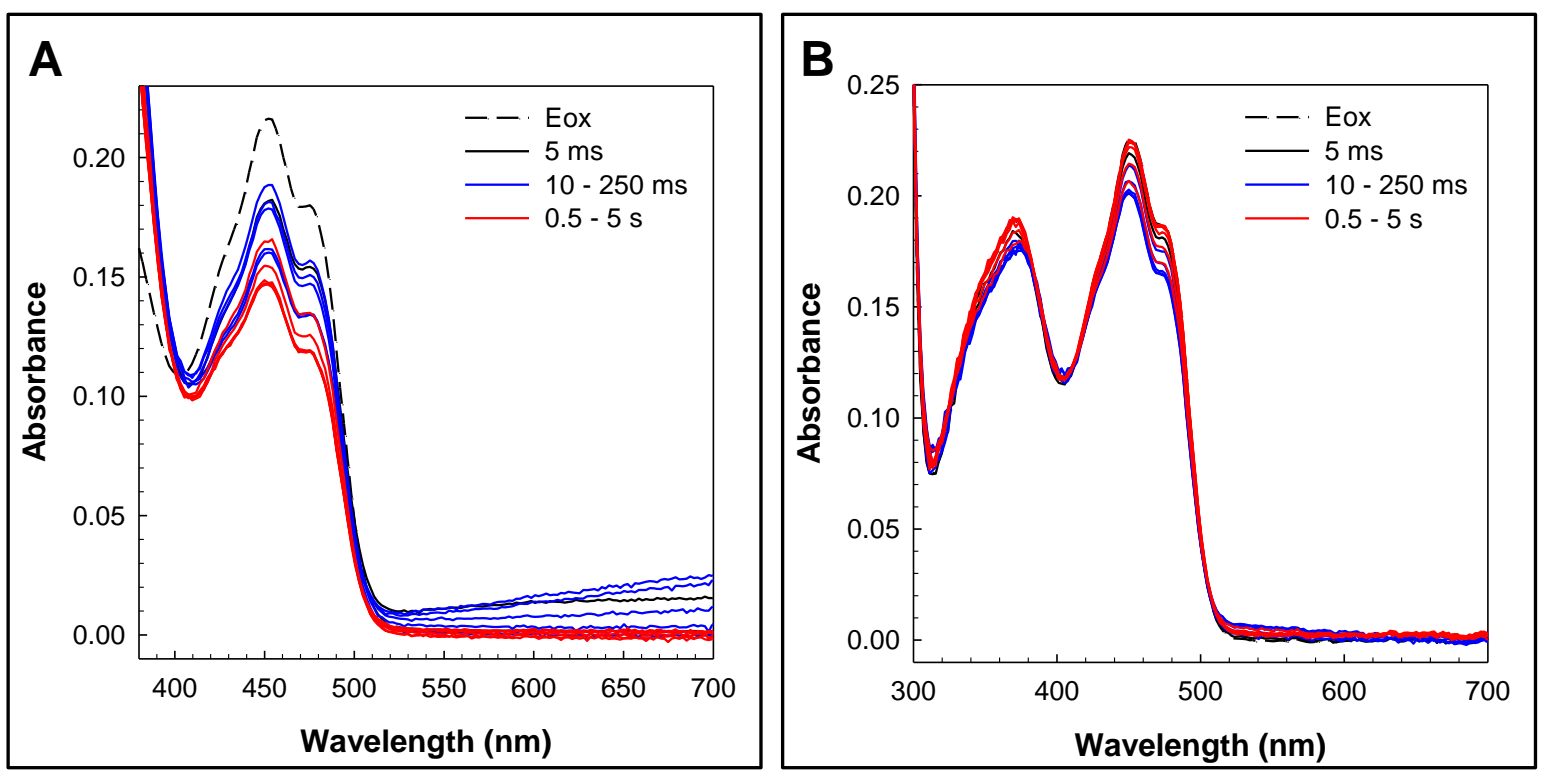

Figure 3.19 Stopped-flow absorbance traces of $E c \mathrm{E} 3 \mathrm{E} 189 \mathrm{Q}$.

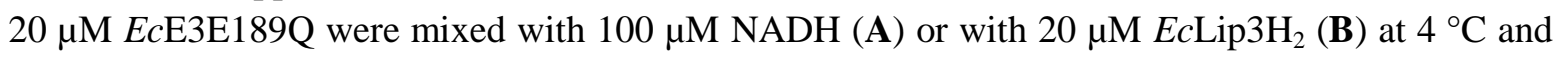
$\mathrm{pH}$ 7.6. The dashed line represents the oxidized form of the enzyme $\left(\mathrm{E}_{\mathrm{ox}}\right)$ derived from a single mixing event with buffer. The solid spectra were recorded using a diode-array detector after mixing at the concentrations indicated. 


\subsubsection{Lys54 and Glu189 are crucial Mediators of the Flavin Redox Potential}

$E c \mathrm{E} 3 \mathrm{~K} 54 \mathrm{Q}$ showed severe overreduction by NADH and a remarkably increased affinity for $\mathrm{NAD}^{+}$. The absence of thiolate-FAD charge transfer complex and the fact that one equivalent of NADH were sufficient for complete flavin reduction indicate that its flavin redox potential is way less negative than that of the wild type enzyme. Mutation of the same residue to arginine raised the FAD potential by approximately $60 \mathrm{mV}$ (Maeda-Yorita et al. 1994), whereas the redox active disulfide potential was unaffected. The results presented here suggest that the redox potential of the FAD has been raised even further and underlie the already proposed sensitivity of the isoalloxazine ring to its electrostatic environment, especially the surrounding of the N5 atom. The positive charge donated by the ammonium group of the lysine residue to the latter atom is thus pivotal for the enzyme. An absolute determination of the flavin redox potential could be achieved using the method which was described in 2.2.2.9 (McDonald et al. 2013).

The role of Lys54 is presumably not limited to its redox-mediating function. It has been suggested that the ammonium group of the lysine repels the positively charged $\mathrm{NAD}^{+}$from the active site in the related human glutathione reductase (Schulz and Pai 1983). The relatively high binding constant for the pyridine nucleotide in $E c \mathrm{E} 3$ might support this function. Further, ITC data suggest that binding of $\mathrm{NAD}^{+}$to K54Q is characterized by an increased change in enthalpy (Figure 5.21). The increased affinity towards $\mathrm{NAD}^{+}$in the variant is therefore most likely based on two aspects: At first, the repulsion of the nucleotide became abolished due to removal of the positive charge at the enzyme and secondly, hydrogen bonding between Gln54 and the nicotine amide part of $\mathrm{NAD}^{+}$might have tightened the interaction.

The mutation of Glu189 to glutamine had the opposite effect with respect to the flavin redox potential. NADH titrations revealed that the potential has been lowered compared to the wild type enzyme. The absence of thiolate-FAD charge transfer complex during both reduction with $\mathrm{NADH}$ and $E c \mathrm{Lip}_{3} \mathrm{H}_{2}$ suggest that the internal transfer of reducing equivalents from the flavin site towards the disulfide and vice versa is almost completely impaired. As observed for Glu450 in the studies on the reductive halfreaction (3.3.1.1), Glu189 presumably acts as structural element that is responsible for positioning the $\varepsilon$-amino group of Lys54 towards the flavin N5 atom. Removal of the carboxylic group of Glu189 might lead to a relaxation of Lys54 from its strained conformation (Figure 3.17) and hence a perturbation of the interaction with N5.

\subsubsection{A direct Involvement of Arg386 in a Proton Transfer Reaction is questionable}

In E. coli mercuric ion reductase the Arg386 homolog (Arg382) is thought to participate in a proton transfer reaction during catalysis by a direct interaction with the catalytic lysine. Most likely this transfer reaction is required to protonate FAD-N5 during the reductive half-reaction to induce covalent 
adduct formation (Susan Miller, University of California, personal communication). Indeed, the fact that Arg386 could be modeled using two alternate conformations implies that this residue harbors certain motility (Figure 3.17) which may indicate that an interaction with Lys54 cannot be totally excluded. However, this would require Lys54 to undergo huge structural rearrangements as well, a scenario which is due to its interactions with Glu189 and FAD-N5 unlikely. In addition, kinetic and spectroscopic data further underpin that replacement of Arg386 reduces the enzyme activity but not to that amount which one would expect if a proton transfer reaction was completely disturbed. Assuming $\sim 1 \%$ residual activity in a case of a fully abolished proton transfer reaction the detected $22 \%$ residual activity are significantly higher. Though it cannot be excluded that a water molecule now occupies the space which became available due to the mutation it remains doubtful that a latter molecule can compensate the complex proposed role of Arg386.

The data presented demonstrate that the mutation raised the redox potential of the flavin already leading to overreduction to the catalytically inactive $\mathrm{EH}_{4}$ state at equimolar concentrations of NADH. However, the steady-state activity shows that $\mathrm{NAD}^{+}$can oxidize the enzyme to the active $\mathrm{EH}_{2}$ state that results in substrate turnover. Further, though the detection of the Cys50-thiolate FAD charge transfer was not possible when NADH served as reducing titrant, $E c E 3 R 386 \mathrm{~A}$ was able to form this complex when EcLip3H2 served, albeit with a smaller amplitude than the wild type. It can be speculated, the less pronounced thiolate FAD charge transfer was masked by the enhanced $\mathrm{NAD}^{+}-$ $\mathrm{FADH}_{2}$ complex during reduction with $\mathrm{NADH}$. These data suggest that $E c \mathrm{E} 3 \mathrm{R} 386 \mathrm{~A}$ is still capable to form all the intermediates required for catalysis and hence the role of $\operatorname{Arg} 386$ has to be seen from a more structural point of view: Mutations at the dimer interface of E3s have the ability to drastically alter the catalytic properties of the enzyme (3.3.1.2 and 3.5). Though the exact reasons for this alteration have not been identified yet, it was speculated that residues at the interface stabilizing the homodimer contribute to optimal catalysis and provide protection against inhibition/overreduction by NADH (Benen, Van Berkel, Veeger, et al. 1992). Since Arg386 is also located at the interface it is likely that the altered observed behavior is due to destabilization of the homodimer rather than a direct involvement in a proton transfer reaction. That the enzyme behavior can indeed be regulated by mutation of a residue present at the dimer interface (Glu354) will be shown and discussed in a following chapter (3.5). 


\subsection{Converting $E c E 3$ into a light-sensitive blue Light Receptor}

A common feature of lipoamide dehydrogenases and LOV proteins is the formation of a covalent FAD-C4a-cysteinyl adduct during the respective reaction cycle. However, according to spectroscopic and structural data (3.1 and 3.2) and previous reports (Argyrou et al. 2002) the covalent adduct only accumulates to a minor fraction in lipoamide dehydrogenases. On the contrary, the lifetime of the same class of adducts can also last up to hours in LOV blue light photoreceptors (Conrad et al. 2014). To therefore extend the lifetime of the adduct in $E c \mathrm{E} 3$ a conversion of this oxidoreductase into a lightsensitive LOV protein using a minimal system was attempted. The aim was to stabilize the adduct upon light activation and by this to overcome its transient nature which prohibited a more detailed spectroscopic and structural study. On the one hand, an accumulation of the C4a-cysteinyl adduct would allow the usage of different spectroscopic analyses and on the other hand further crystallographic studies, which may expand the gained knowledge about this reaction central intermediate during the course of this study. Further, this structure-guided protein engineering approach should combine the catalytic properties of a LOV protein and the $E c E 3$ enzyme leading to a blue-light absorbing oxidoreductase capable of reducing NADH with light as the main driving force. The envisaged reaction cycle would include a photochemical induced $\mathrm{C} 4 \mathrm{a}$-cysteinyl adduct followed by binding of $\mathrm{NAD}^{+}$to generate NADH. The required reduction of the thiol would be achieved by an externally added sulfhydryl-free reducing agent (TCEP).

\subsubsection{EcE3C45A/K54Q and C45S/K54Q - PseudoLOV variants}

Based on the structural and functional similarities of both protein classes minimal systems were created in which the active site of $E c E 3$ was modified to mimic that of a LOV protein. Whereas LOV domains harbor a conserved cysteine residue close to the FMN cofactor lipoamide dehydrogenases exhibit a redox active cystine (Figure 3.20). Thus, mono-thiol variants of $E c \mathrm{E} 3$ were generated for the required active site modification. As aforementioned, UV-Vis spectroscopic data suggested that Cys50 in $E c \mathrm{E} 3 \mathrm{C} 45 \mathrm{~A}$ is present in its thiol form (3.1.5) and, moreover, is capable of $\mathrm{NAD}^{+}$-induced adduct formation (Figure 3.5 and Figure 5.2). Hence, EcE3C45A seemed to be a suitable starting point for the establishment of a pseudoLOV minimal system. In another mono-thiol variant, EcE3C45S, the remaining cysteine (Cys50) is present in its anionic form leading to a permanent charge transfer complex between the Cys50-thiolate and the flavin cofactor (3.1.4). Since the charge transfer complex is on-pathway in the reaction cycle of the native enzyme this variant was chosen as second mono-thiol variant. Besides targeting the cystine moiety as a site for mutations, another residue crucial for $\mathrm{H}$ bonding with FAD-N5 during the respective reaction cycle is different in both enzymes and was therefore the second target for site-directed mutagenesis. A highly conserved glutamine residue hydrogen bonds to flavin N5 in LOV proteins and has a key role in transducing the light signal 
(Conrad et al. 2014) whereas a conserved lysine residue is thought to facilitate N5 protonation during the reaction cycle and to mediate the FAD redox potential (Maeda-Yorita et al. 1994). Thus, Lys54 was mutated to Gln54 leading to the pseudo LOV proteins EcE3C45A/K54Q (pseudoLOV) and $E c \mathrm{E} 3 \mathrm{C} 45 \mathrm{~S} / \mathrm{K} 54 \mathrm{Q}$ (thiolateLOV) (Figure 3.20).

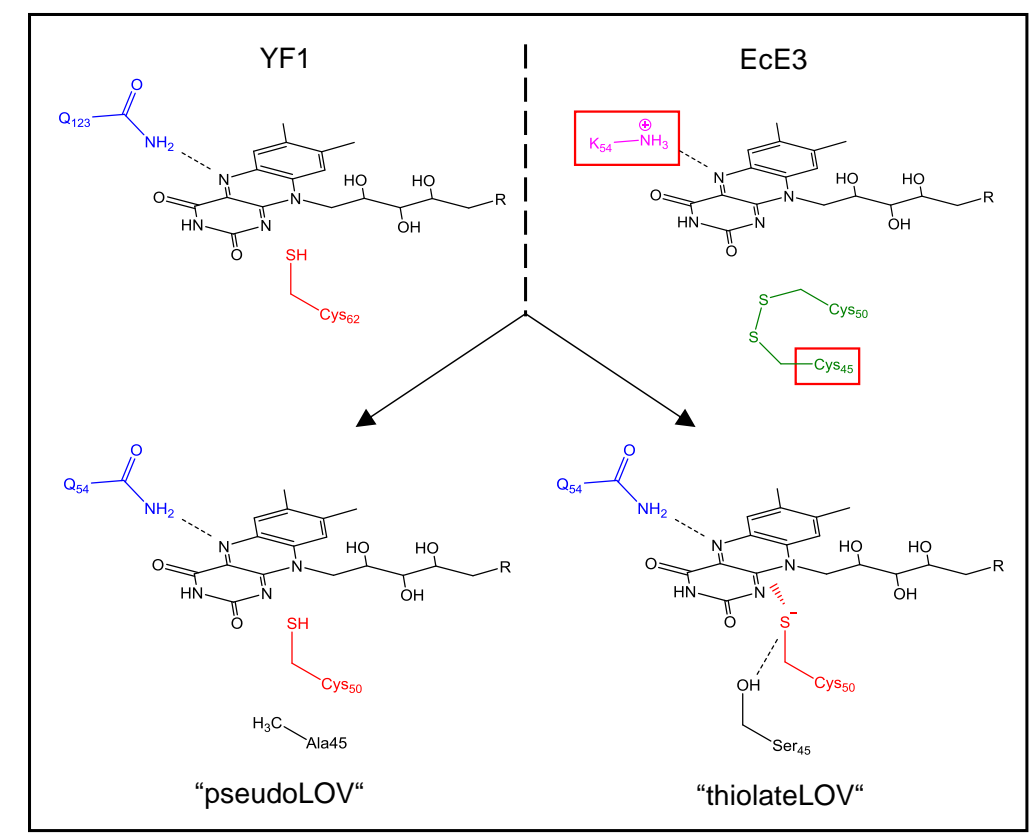

Figure 3.20 Converting $E c E 3$ into a pseudoLOV protein.

(top) Schematics of the active sites of YF1 and EcE3. YF1 numbering is taken from PDB 4GCZ. Targeted residues during mutagenesis are marked with a red box. (bottom) Schematics of the "pseudoLOV" variants $E c E 3 C 45 \mathrm{~A} / \mathrm{K} 54 \mathrm{Q}$ and $E c \mathrm{E} 3 \mathrm{C} 45 \mathrm{~S} / \mathrm{K} 54 \mathrm{Q}$. For detailed description of the chosen mutations see text.

Both double-variants could be purified to homogeneity and their structural integrity was verified by far-UV CD spectra (5.6). In addition, both pseudoLOV proteins retained their typical color (yellow and orange-red, respectively) which was also observed for the respective single variant. UV-Vis ground-state spectra proofed that substitution of Lys54 did not change the respective protonation state in each mono-thiol variant (5.2D and E). Thus, the pseudo- and the thiolateLOV variant fulfill the requirements to reflect a minimal LOV protein system. 


\subsubsection{Photobleaching of pseudoLOV Variants leads to irreversible Alterations at the Flavin Site}

In order to investigate the photochemical response of re-engineered E3 variants the proteins were subjected to blue-light exposure with concomitant read out on a photodiode array. Exposure of $E c \mathrm{E} 3 \mathrm{C} 45 \mathrm{~A} / \mathrm{K} 54 \mathrm{Q}$ to blue light $\left(455 \mathrm{~nm}, 50 \mathrm{~mW} / \mathrm{cm}^{-2}\right)$ changed the absorption properties, indicating that the protein is photochemically reactive. The typical flavin band at $450 \mathrm{~nm}$ decreased during the time course with concomitant increase in the lower wavelength region at 340 to $350 \mathrm{~nm}$ showing an isosbestic point at $380 \mathrm{~nm}$ (Figure 3.21A). The final spectrum is different to that of a fully reduced flavin (Ghisla et al. 1974). Recovery kinetics showed that the absorbance change is irreversible. Photoreduction EcE3C45S/K54Q under the same conditions lead to comparable absorbance changes but with less drastic effects. However, the tendency of a decrease in absorbance in the longer wavelength range and increase in lower range is evident. As for the thiol variant these processes were irreversible. Noteworthy, the thiolate charge transfer between Cys50 and chromophore was only slightly affected (Figure 3.21B). In both variants, a significant increase in absorbance between $380-$ $390 \mathrm{~nm}$ was missing leading to the assumption that formation of a cysteinyl-C4a adduct is unlikely.

Control experiments were performed to determine, at first, the photoreactivity of $E c \mathrm{E} 3 \mathrm{wt}$ towards blue light and secondly to verify whether the observed absorbance changes were due to adduct formation involving the flavin N5 atom. It was demonstrated that light induced N5-adducts harbor a comparable spectroscopic signature as described above (Ghisla and Massey 1975) and therefore the investigation of a putative interaction of Cys50 with N5 was of special interest. The results obtained for the controls can be found in the appendix (5.7). EcE3 wild type also showed a certain photochemical reactivity but with a much lower extent than the pseudoLOV variant. Further, the formation of covalent N5-adducts is unlikely since the mono- or non-thiol variants showed comparable spectroscopic changes upon light illumination: mutation of Cys50 facing the flavin cofactor to either alanine ( $E c \mathrm{E} 3 \mathrm{C} 50 \mathrm{~A})$ or serine $(E c E 3 C 50 S)$ made adduct formation unlikely due to steric or chemical reasons. To further exclude a covalent bond formation between protein and cofactor during photobleaching the proteins denatured with $6 \mathrm{M}$ guanidinium chloride which lead to a release of the chromophore in all cases. The formation of a flavin radical species during photobleaching is also unlikely since the UV-Vis signatures did not show specific bands for neither the neutral nor the anionic semiquinone radical (Choong and Massey 1980; Massey and Palmer 1966). Time-resolved EPR measurements confirmed that radical species do not accumulate to an significant amount (5.8).

Low intensity $\left(5 \mathrm{~mW} / \mathrm{cm}^{-2}\right)$ blue light illumination of LOV domain YF1 (Diensthuber et al. 2013) drastically leads to changes in the UV-VIS signature of the protein. In the dark state, the flavin is fully oxidized having an absorption maximum at $447 \mathrm{~nm}$. Formation of the flavin-(C4a)-cysteinyl covalent bond blue-shifts the absorbance to a new maximum at $390 \mathrm{~nm}$ (Figure 3.21C). Ten-fold higher light intensities $\left(50 \mathrm{~mW} / \mathrm{cm}^{-2}\right)$ lead to the same spectroscopic changes and, more importantly, the lit state 
spectrum was stable for at least one hour during photobleaching under the harsher conditions (personal communication by Prof. Andreas Möglich). In contrast, YF1C62A, a variant in which the bondforming cysteine residue is replaced by an alanine, was not resistant against long-term photobleaching (Figure 3.21D). The spectroscopic changes for this enzyme were similar to those observed for the pseudoLOV protein: a decrease of the main flavin peak with a simultaneous increase in the lower wavelength region, with an isosbestic point at $380 \mathrm{~nm}$. However, in contrast to the pseudoLOV protein a broad absorption band with maxima at 570 and $610 \mathrm{~nm}$ developed during photobleaching. This spectral property is similar to those of other flavoprotein neutral radicals (Massey and Palmer 1966; Talfournier et al. 2001a). Radical formation came along with loss in absorbance at $447 \mathrm{~nm}$ but then also diminished during the further bleaching process leading to a single peak formation at $\sim 330 \mathrm{~nm}$. The entire bleaching process was also irreversible. 

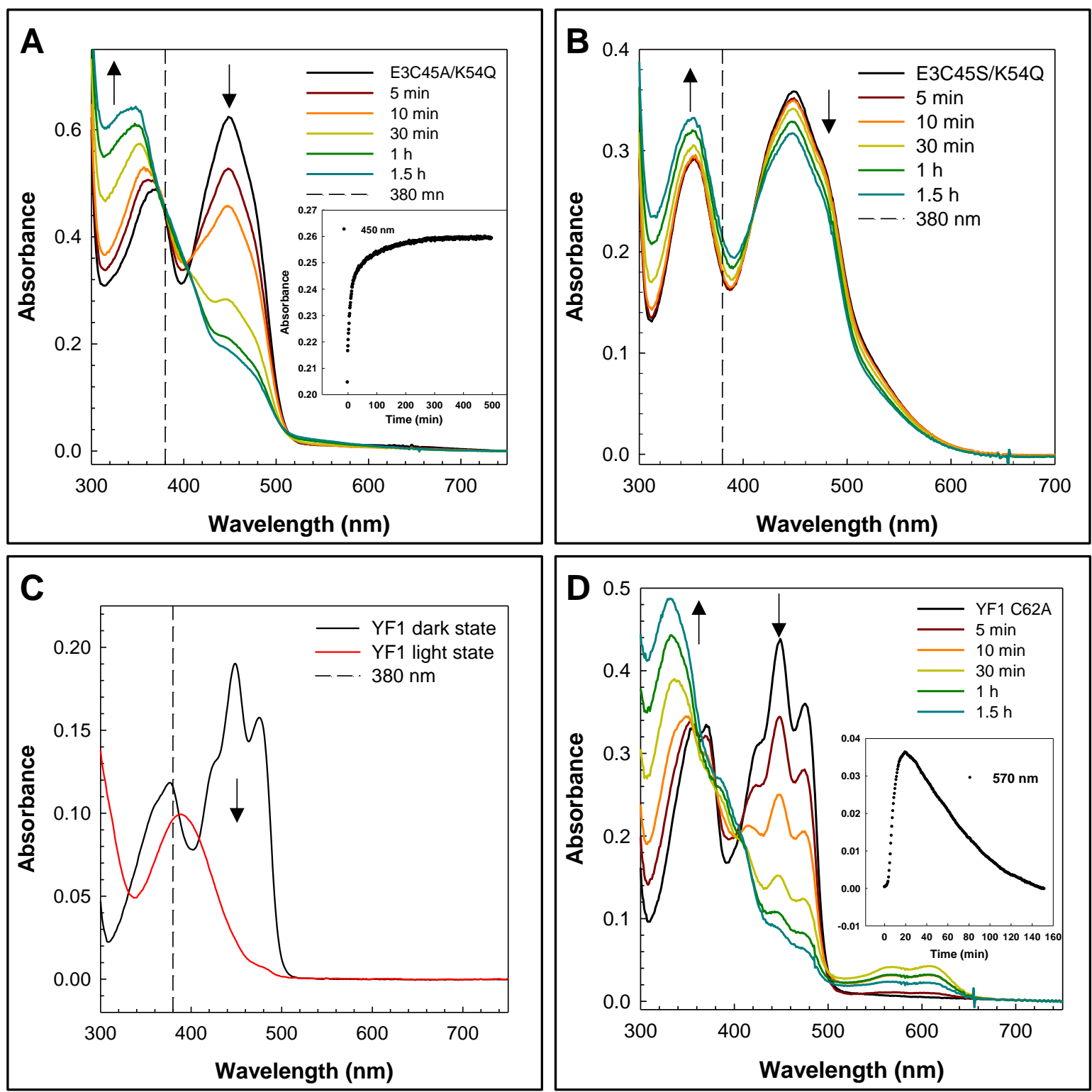

Figure 3.21 Comparison of abosorbance changes in variants of pseudoLOVs and LOV domains upon blue light illumination.

UV-Vis spectra of pseudoLOV (A) and thiolateLOV (B) during photobleaching $\left(50 \mathrm{~mW} / \mathrm{cm}^{-2}\right)$ with blue light $(455 \mathrm{~nm})$. The inset shows the recovery kinetic in the dark. (C) UV-Vis absorbance profiles of YF1 wild-type protein. The black trace represents the dark state spectrum and red trace the lit state spectrum. Data were kindly provided by Prof. Andreas Möglich, HU Berlin. (D) UV-Vis spectra of YF1C62A during photobleaching $\left(50 \mathrm{~mW} / \mathrm{cm}^{-2}\right)$. The inset shows formation and decay of the neutral semiquinone radical at $570 \mathrm{~nm}$. 


\subsubsection{Mass Spectrometric Analysis of the photobleached Flavin Cofactor}

To further elucidate the alterations occurring at the flavin site upon light illumination the photobleached cofactor of pseudoLOV $(E c \mathrm{E} 3 \mathrm{C} 45 \mathrm{~A} / \mathrm{K} 54 \mathrm{Q})$ was isolated from the enzyme and subjected to both UPLC-ESI-TOF-MS and UHPLC-ESI-QTOF-MS/MS. MS analysis of FAD (purchased from AppliChem) and of isolated cofactor from unbleached pseudoLOV served as reference measurements. The usage of three different isolation techniques and their subsequent analysis excluded that modifications at the flavin site were due to the respective isolation method (2.2.6.5). All MS spectra belonging to the reference measurements and all spectra for the different isolation techniques for photobleached and unbleached samples are shown in the Appendix (5.9).

UPLC-ESI-TOF-MS analysis of the photobleached flavin cofactor showed the presence of two additional flavin derivatives besides the non-modified FAD (Figure 3.22). The derivative eluting slightly earlier than native FAD has an $m / z 782.13$ and the corresponding UV-Vis spectrum shows maxima at 367 and $450 \mathrm{~nm}$ (Figure 3.22C and D, blue line). The lower $\mathrm{m} / \mathrm{z}$ ratio of two protons compared to FAD $(m / z 784.18)$ can be explained by a double bond formation whereas a localization of the latter in the isoalloxazine part is unlikely since the UV-Vis spectrum reflects the shape of an nonmodified FAD. According to the results obtained this derivative is termed $F A D-2 H$. A more detailed investigation to localize the putative double bond is shown later (Figure 3.24).

The retention time of the second derivative is shifted to a later time point with an $m / z 241.07$ and the UV-Vis spectrum harbors a maximum at $350 \mathrm{~nm}$ (Figure 3.22C and D, red line). The latter derivative was identified as lumichrom by comparing the data with the data obtained from a lumichrom standard solution (Figure 5.12 and 5.13). This indicates the formation of lumichrom during photobleaching of pseudoLOV. 

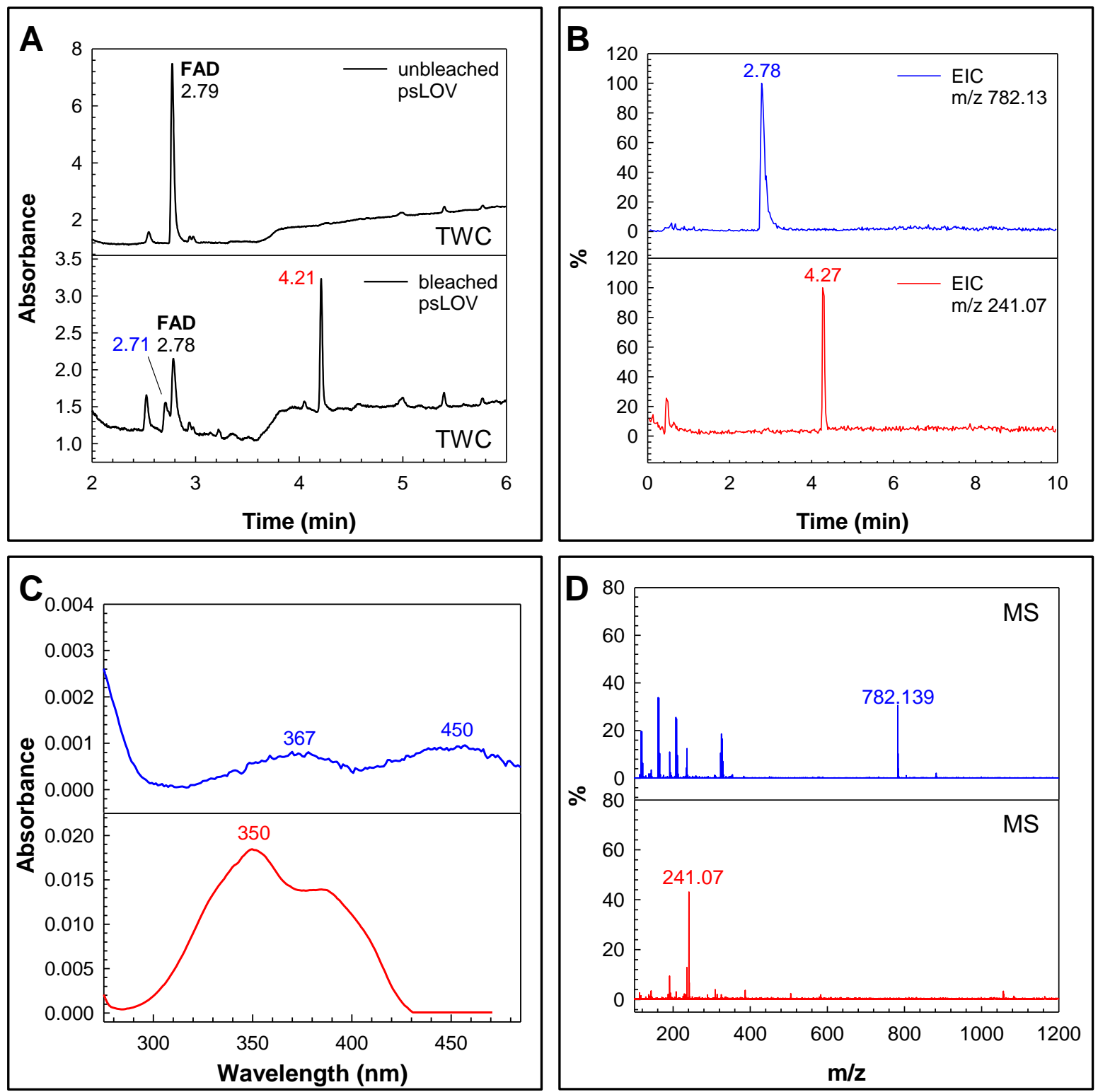

Figure 3.22 UPLC-ESI-TOF-MS analysis of cofactors of bleached and unbleached psLOV.

(A) Total wavelength chromatogram of TCA extracted cofactors from unbleached (top) and photobleached (bottom) pseudoLOV. Corresponding UV-Vis spectra are shown in (C) with the respective color. (B) Extracted ion chromatogram for $m / z 782.13$ (top) and $m / z 241.07$ (bottom) and corresponding MS analysis (D) in negative ionization mode. Allocation of FAD is based on the analysis of an FAD standard solution shown in the appendix (Figure 5.8).

Besides the formation of lumichrom and $F A D-2 \mathrm{H}$ a third derivative could be identified during UPLCESI-TOF-MS analysis (Figure 3.23). This derivative was not shown in Figure 3.22 since the TCA used for cofactor extraction eluted in the same time scale making a UV-Vis detection impossible. The derivative has an $\mathrm{m} / \mathrm{z} 542.07$ and the UV-Vis spectrum shows a single peak with its maximum at $260 \mathrm{~nm}$. This UV-Vis signature is typical for an adenine moiety indicating that this FAD derivative lost its isoalloxazine part. The latter is almost congruent with the obtained mass, however a formation of an additional double bond (as described for $F A D-2 H$ ) is required to achieve the appropriate 
observed mass. According to the results obtained this derivative is tentatively assigned as ribityl-ADP$2 H$.
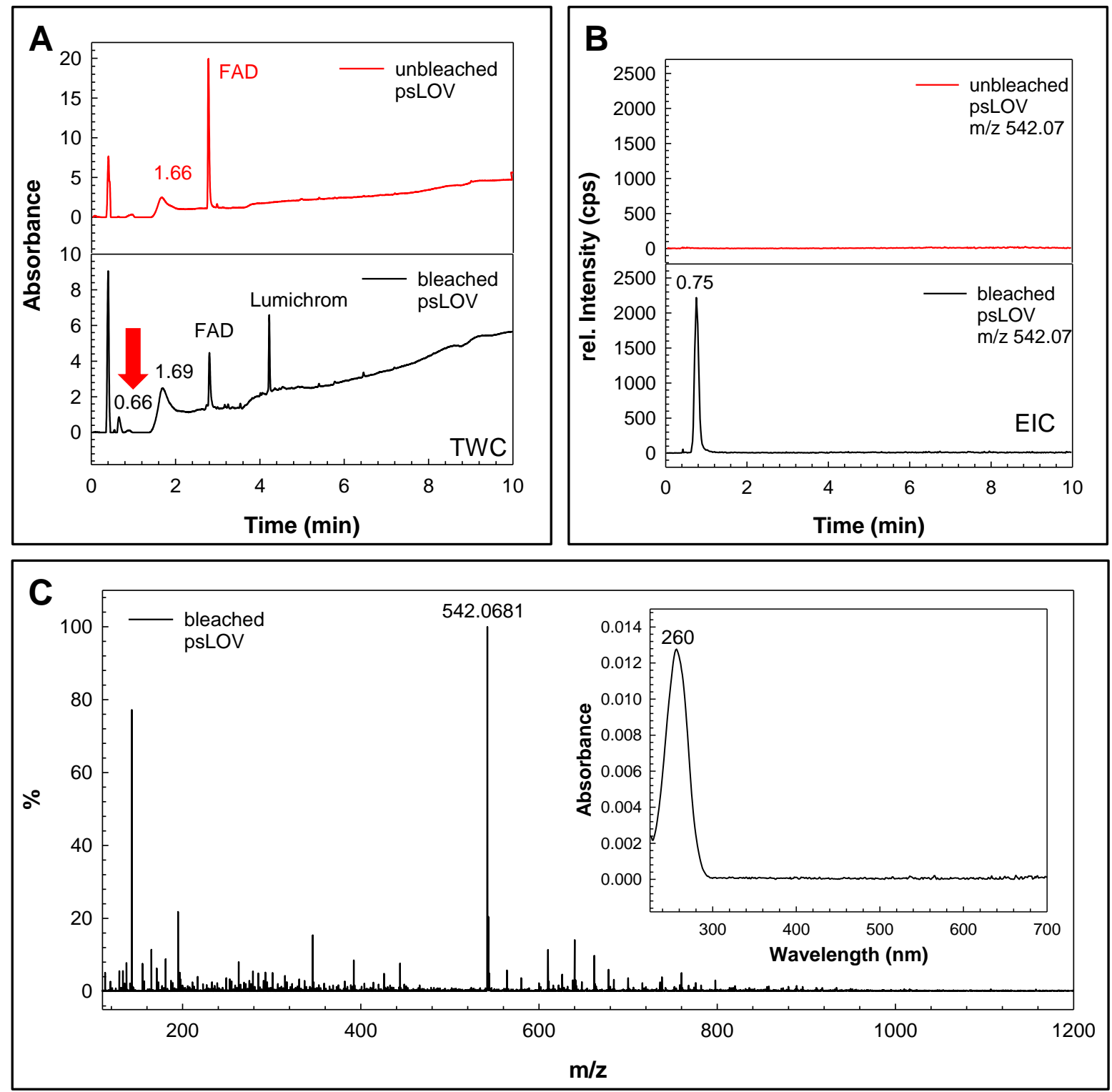

Figure 3.23 UPLC-ESI-TOF-MS analysis of ribityl-ADP- $2 H$.

(A) Total wavelength chromatogram of heat extracted cofactors from unbleached (top) and photobleached (bottom) pseudoLOV. (B) Extracted ion chromatograms for $m / z 542.07$, respectively. (C) MS analysis (negative ionization mode) of $\mathbf{B}$ and corresponding UV-Vis spectrum of (inset). Allocation of FAD and lumichrom is based on the analysis of standard solutions shown in the appendix (Figure 5.8 and Figure 5.12).

UPLC-ESI-TOF-MS analysis thus far revealed the presence of three additional FAD derivatives formed upon photobleaching of pseudoLOV: lumichrom, $F A D-2 H$ and ribityl-ADP-2H. On the one hand, lumichrom could clearly be identified on the other hand, the exact position of the double bond in 
the two remaining derivatives is unclear. The double bond position was then investigated by subjecting FAD and the $F A D-2 H$ derivative to UHPLC-ESI-QTOF-MS/MS. The interpreted fragmentation pattern indicates that no double is formed in neither the isoalloxazine, nor in the AMP part of the FAD since the respective fragments show the same $m / z$ values (Figure 3.24, black arrows). This indicates that the double bond is positioned in the ribityl part which is consistent with the UV-Vis spectroscopic data obtained by UPLC-ESI-TOF-MS. The UV-Vis signature of $F A D-2 H$ and of ribityl$A D P-2 H$ resemble the one of FAD and an adenine moiety, respectively. Double bond formation in the ribityl part led to an altered MS/MS fragmentation pattern. While two fragments deviate by two protons $(\mathrm{m} / \mathrm{z} 517.05 / 515.04$ and $\mathrm{m} / \mathrm{z} 437.08 / 435.07)$ one further fragment deviates by an additional carbon atom $(\mathrm{m} / \mathrm{z} 180.99$ / 192.99). Moreover, the fragment with an $\mathrm{m} / \mathrm{z} 337.09$ is exclusive for ribityl$A D P-2 H$.
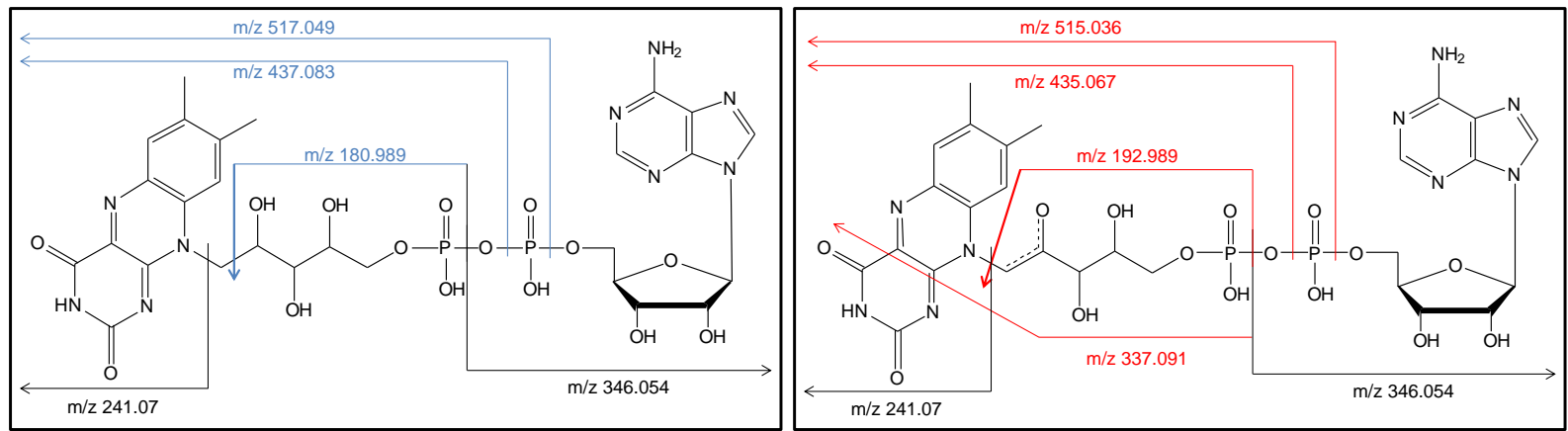

Figure 3.24 The double bond in $F A D-2 H$ and in ribityl-ADP-2H is located the ribityl part.

MS/MS fragmentation pattern at $20 \mathrm{eV}$ collision energy for FAD at $m / z 784.15$ (left) and for FAD-2H at $m / z 782.13$ (right). FAD derivatives were interpreted in comparison to FAD spectra (METLIN ID2302) deposited in the METLIN database. Fragments harboring the same $\mathrm{m} / z$ ratios are indicated in black, different ratios are shown in blue and red, respectively. The fragmentation patterns indicate that the modified position is housed in the ribityl part of the FAD derivative. The MS/MS fragmentation pattern is shown in (Figure 5.16).

\subsubsection{C4a-cysteinyl Adduct Formation in LOV Domains prevents Photodegradation of the Flavin Cofactor}

The results obtained so far showed that the generated pseudoLOV minimal systems were not sufficient to promote light-driven cysteinyl-C4a adduct formation. A further spectroscopic and crystallographic investigation of these adducts was therefore not possible. However, the experiments offer a new perception regarding the LOV protein photocycle and photodegradation of enzyme-bound flavin cofactors. The data obtained for YF1 and its active site variant YF1C62A lead to the assumption that 
covalent bond formation between the conserved cysteine residue and the FMN cofactor during the photocycle harbors an intrinsic protective function against photodegradation. As described above, high-intensity photobleaching of the wild type LOV domain did not influence the lit state spectrum while the same intensities altered the spectral properties of the $E c \mathrm{E} 3$ wild type and its pseudo LOV derivatives. This spectral change is based on irreversible alterations at the flavin site (3.4.2). Hence, there must be a mechanistic or structural property preventing LOV domains from photodegradation and thus keeping the protein active to perform further rounds of photocycle and concomitant signal transduction. Mutation of the bond forming cysteine residue to alanine (YF1C62A) abolished this resistance leading to spectroscopic changes similar to those obtained for the oxidoreductase (Figure 3.21D). Therefore, bond formation between a cysteine and flavin cofactor seems to be the crucial part of the protective function against photodegradation. C4a-cysteinly adduct formation leads to loss of aromaticity of the flavin ring system and thereby changes its electronic configuration. This results in a blue shifted spectrum and reduces absorption of light with longer wavelengths ( $>400 \mathrm{~nm}$ ). It was demonstrated that longer wavelengths, especially in the range of $415-455 \mathrm{~nm}$, drastically damage riboflavin in solution (Sattar, deMan, and Alexander 1977). Since the flavin adduct does not absorb light with this particular wavelength a relation between the latter and resistance against photodegradation is evident. Thus, LOV domains have evolved a structural feature which simultaneously permits both signal transduction on the one hand and protection against photodegradation on the other (Figure 3.25).

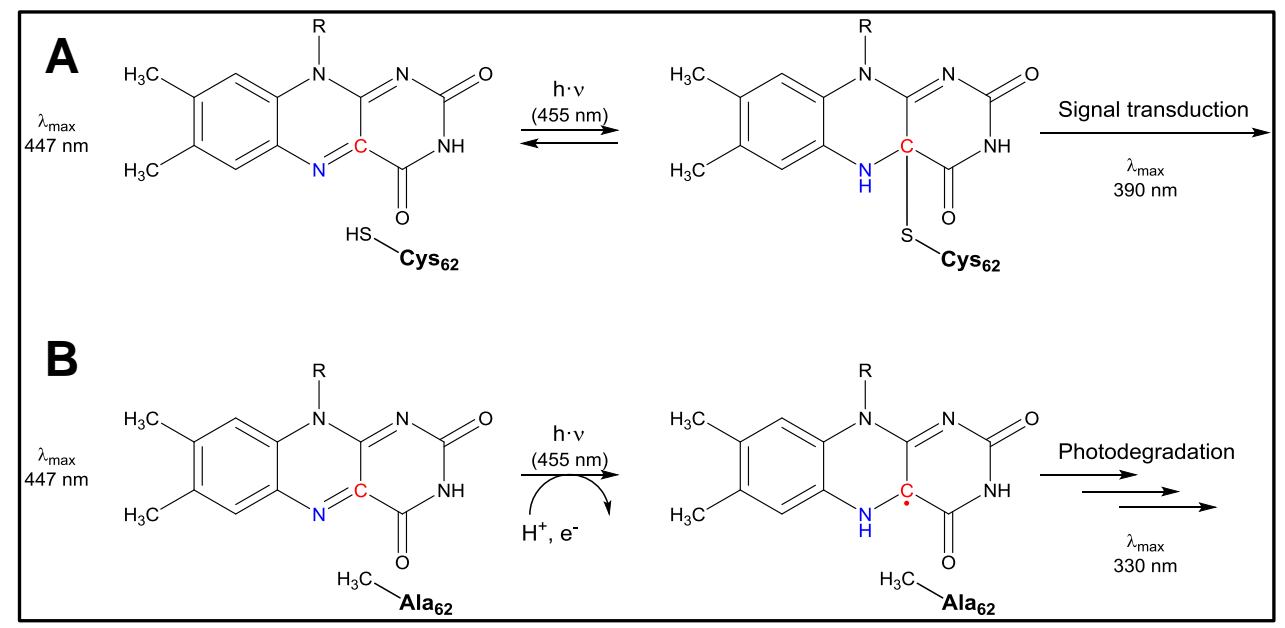

Figure 3.25 Protective mechanism of LOV proteins.

(A) C4a-cysteinyl adduct formation exhibits two functions: Signal transduction and protection against photodegradation. (B) Blue light exposure of the cysteine-lacking variant induces formation of the neutral semiquinone, the potential pre-stage prior to photodegradation. 
The formation of the flavin neutral semiquinone radical in YF1C62A was the major difference during photobleaching of the cofactor compared to the pseudoLOV protein. Though, the single peaks are blue-shifted by $\sim 10 \mathrm{~nm}$, the position of the radical band maxima are similar to those observed for glucose oxidase, DNA (6-4) photolyase or an animal-like cryptochrome (aCRY) (Beel et al. 2012; Hitomi et al. 1997; Massey and Palmer 1966). Since there is evidence for a neutral semiquinone intermediate between the excited triplet and the adduct state (Bauer et al. 2011) the accumulation of a neutral radical in the cysteine-missing variant is not unexpected. However, it remains unclear whether formation of the neutral radical lies on pathway, and thus accumulates since adduct formation is impaired, or whether radical formation is a side effect of the photodegradation of the FMN cofactor. According to the rate of the semiquinone formation the latter is more likely since radical formation in aCRY is 40-fold faster and, even more important, reversible (Beel et al. 2012). To compare the putative influence of the semiquinone radical on the photodegradation a mass spectrometric analysis according to the pseudoLOV protein should be performed (see discussion below).

\subsubsection{Photodegradation of enzyme-bound FAD leads to Double Bond formation in the ribityl part}

Mass spectrometric analysis revealed three major FAD derivatives as photodegradation products upon blue light exposure of the pseudoLOV protein: lumichrom, $F A D-2 H$ and ribityl-ADP-2H (Figure 3.26) whereas the formation of lumichrom was confirmed by reference measurements. Photostability and degradation of riboflavin in aqueous solvents have been extensively studied due to its relevance in food products and pharmaceutical preparations. It is degraded into several photoproducts including the aforementioned lumichrom, but also into formylmethylflavin, lumiflavin, carboxymethylflavin and further derivatives (Sheraz, Kazi, Ahmed, Anwar, et al. 2014). Formation of lumichrom is controversially discussed and both excited singlet and excited triplet states of riboflavin are stated to be precursors in the photodegradation reactions by different mechanisms. On the one hand, lumichrom is formed by the excited triplet state (Ahmad et al. 2004; Huang, Hyun, and Min 2006; Jung et al. 2007) on the other hand, the excited singlet was also claimed as a precursor (Ahmad et al. 2004; Cairns and Metzler 1971; Sheraz, Kazi, Ahmed, Mirza, et al. 2014). However, the distinction between these reactions in the photodegradation process lacks detailed information (Sheraz, Kazi, Ahmed, Anwar, et al. 2014). To get information about the excitations states of the enzyme-bound cofactor nanosecond time-resolved UV-Vis spectroscopy by flash photolysis should be performed (Bauer et al. 2011). This would potentially allow a comparison of the flavin excitations states generated in solution and when bound to an enzyme.

Though information in the nano- and microsecond time regime of the photobleaching event of the pseudoLOV protein are missing alterations of the degradation profile compared to riboflavin in solution are obvious. First, it has been described that the presence of divalent anions, such as 
phosphate $\left(\mathrm{HPO}_{4}{ }^{2-}\right)$ and sulfate $\left(\mathrm{SO}_{4}{ }^{2-}\right)$, exclusively lead to the formation of cyclodehydroriboflavin as phtotodegradation product (Ahmad et al. 2010; Schuman Jorns, Schöllnhammer, and Hemmerich 1975; Sheraz, Kazi, Ahmed, Mirza, et al. 2014). This product was not identified during MS analysis and thus an influence of the used buffer system $(50 \mathrm{mM} \mathrm{KPP} \mathrm{pH} \mathrm{7.6,} 100 \mathrm{mM} \mathrm{NaCl})$ on the degradation process of the enzyme-bound flavin is unlikely. Moreover, among the plethora of riboflavin degradation products thus far described, out of the three derivatives formed lumichrom was the only one which has yet been reported. Hence, an influence of the enzyme surrounding on the photodegradation process is evident. The ultimate structure of the two remaining derivatives $(F A D-2 H$ and ribityl-ADP-2H) could not be determined since information about the absolute position of the light-induced double bond is missing (Figure 3.24). Formation of the latter fragments lead to the assumption that the double bond is delocalized between the $\mathrm{C} 1, \mathrm{C} 2$ and $\mathrm{C} 2=\mathrm{O}$ atoms of the ribityl part (Figure 3.26). This assumption is on the one hand underpinned by the UV-Vis spectroscopic signatures of both fragments indicating no influences of the double bond on the isoalloxazine nor the adenine moiety of FAD (Figure 3.22C and Figure 3.23C) and on the other hand by the altered MS/MS fragmentation pattern. A delocalized double bond between the $\mathrm{C} 1, \mathrm{C} 2$ and $\mathrm{C} 2=\mathrm{O}$ atoms might explain the altered fragmentation pattern around $\mathrm{C} 1$ and thus the presence of the one-carbon-elongated fragment with an $m / z$ 192.99. Further, localization of the double bond in the mentioned region could result in a stabilization of ribityl-ADP- $2 H$. Assuming an additional bond formation between $\mathrm{C} 2$ and $\mathrm{C} 2=\mathrm{O}$ the formation of a pseudo-six-membered ring system could be the consequence. Here the hydrogen atom the $\mathrm{C} 4-\mathrm{OH}$ group could hydrogen bond to $\mathrm{C} 2=\mathrm{O}$ (Figure 3.26). It cannot be ruled out whether $F A D-2 H$ is a potential precursor of ribityl-ADP- $2 H$. Therefore, MS analysis of photobleached samples taken at different time points could provide further insights into the photodegradation process of enzyme-bound flavin. 


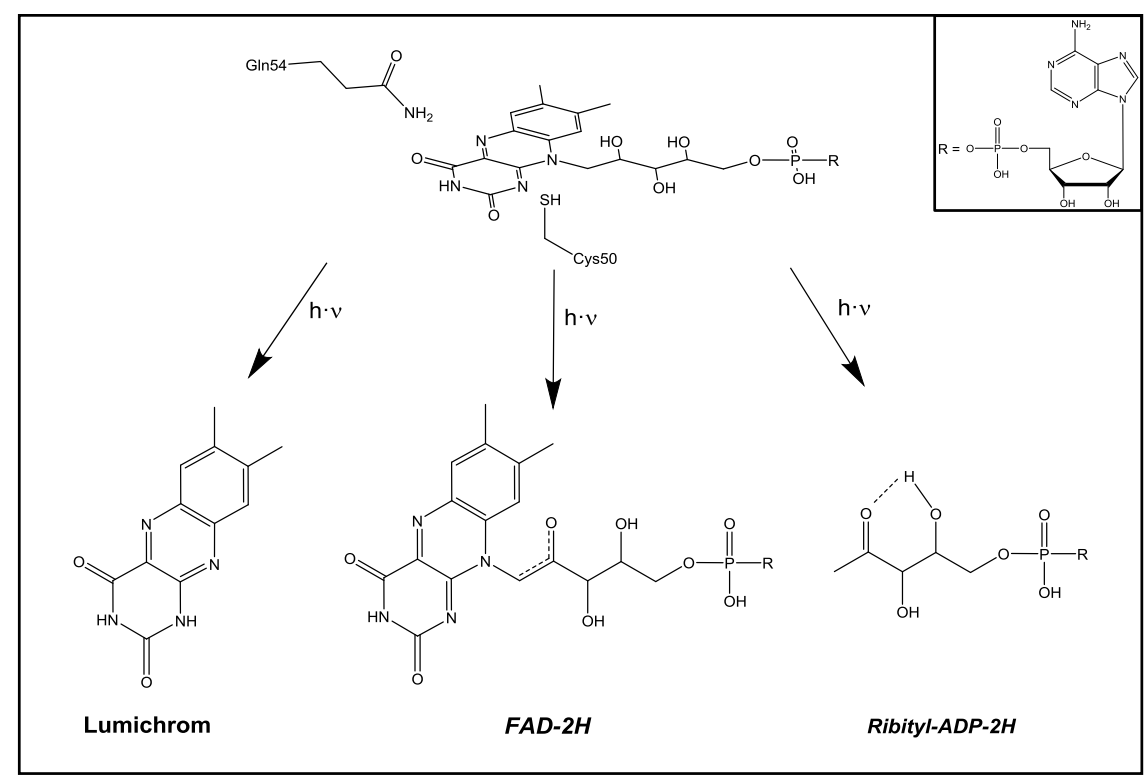

Figure 3.26 Putative FAD derivatives formed upon pseudoLOV photobleaching.

The presence of lumichrom was verified by reference measurements with a lumichrom standard solution. The shown structures for $F A D-2 H$ and ribityl-ADP- $2 H$ are hypothetical (see discussion). The inset shows the AMP part of the FAD derivatives which is not modified during bleaching. 


\subsubsection{The H-bonding Network around the Flavin - The next Step on the Way to a light-sensitive Oxidoreductase?}

The studies on the re-engineered E3 variants have not yet yielded a light-driven system for production of reduction equivalents yet, but they have provided key insights into the inner workings of both E3 metabolic enzymes and LOV photoreceptors (3.4.3.1 and 3.4.3.2). The used minimal system, in which the redox active cystine was converted into a monothiol moiety and in which the residue crucial for (de)protonation of FAD-N5 was exchanged (Lys54 $\rightarrow$ Gln54), was thus too rudimentary to convert the oxidoreductase into a light-sensing protein. Hence, there must be additional factors which are required for light-driven adduct formation. The redox potential of the flavin cofactor in the LOV kinase LOVk from C. crescentus was determined to be $-258 \mathrm{mV}$ (Purcell et al. 2010) which is way more positive than the reported $-314 \mathrm{mV}$ in $E c E 3 w t$ (Maeda-Yorita et al. 1991). However, redox titrations with the pseudoLOV protein $(E c \mathrm{E} 3 \mathrm{C} 45 \mathrm{~A} / \mathrm{K} 54 \mathrm{Q})$ revealed a redox potential of $-268 \mathrm{mV}$ (Table 5.2) which is strikingly close to the reported potential of the cofactor in LOVk. The elevation is thereby mainly related to the replacement of the N5 interacting lysine by a glutamine rather than the presence of the thiol at the si face of the cofactor (3.3.2.1). Though the tendency of the flavin to gain electrons was eased und thus the interaction of the Cys50 thiol moiety with the $\mathrm{C} 4 \mathrm{a}$ atom should have been facilitated it is obvious that further features are required to promote adduct formation. It was demonstrated that the extended hydrogen bond network stabilizing the FMN cofactor in LOV domains (Figure 3.27A) can modulate the quantum yield, kinetics, and thermodynamics of the photocycle (Raffelberg et al. 2011). Consequently, a further step to convert $E c E 3$ into a light-sensing protein should target the surrounding of the chromophore in the oxidoreductase (Figure 3.27B). Putative candidates to alter the microenvironment around the pteridine part of the EcE3 FAD are His445 and Ala321 which could be exchanged with uncharged, polar amino acids. However, negative side effect altering the FAD's redox potential too much have to be considered. Redox titrations for further variants are hence obligate.

Photobleaching of YF1C62A demonstrated that the LOV protein is capable of stabilizing the neutral flavin semiquinone radical if adduct formation is impaired (Figure 3.25). Taking into account that such species was identified between the triplet and the adduct state in a wild type enzyme (Bauer et al. 2011) it is most likely that these photoreceptors provide a surrounding which relieves neutral semiquinone formation. This is further underpinned by the finding that a replacement of the conserved asparagines interacting with the pteridine part of the FMN with negatively charged aspartates did not affect the dynamics and energetics of adduct formation (Raffelberg et al. 2011). According to the authors the latter would be unlikely if a transiently charged species at the flavin site would occur during photocycle. Furthermore, FTIR techniques have provided evidence that the triplet state of FMN is unprotonated making an ionic mechanism (Alexandre et al. 2009; Pfeifer et al. 2009) even more questionable. Though there are multiple evidences that adduct formation might proceed through an 
ionic intermediate (Kennis and Groot 2007; Losi and Gärtner 2011; Zoltowski and Gardner 2011) the results presented here indicate that a radical pair mechanism via a triplet-state $\mathrm{FMNH}^{\circ}-\mathrm{H} 2 \mathrm{CS}^{\circ}$ biradical yielding the covalent bond between cofactor and cysteine still has to be considered. To demonstrate that neutral semiquinone formation in YF1C62A is not a side effect of the degradation of the cofactor, the method established by BAUR et al. using nanosecond flash photolysis with CCD camera detection should be applied to the active site variant (Bauer et al. 2011). This could proof that formation of the blue flavin radical is also possible in a transient time regime. However, a crucial limitation of the active site mutant is evident: the conserved Cys, the likely candidate for the proton source required for N5 protonation during neutral semiquinone formation (Alexandre et al. 2009; Kennis and Groot 2007; Losi and Gärtner 2011; Pfeifer et al. 2009; Zoltowski and Gardner 2011) is absent. It is hence possible that flash photolysis experiments do not show a transient formation of the neutral semiquinone and that the observation of the latter during the steady-state photobleaching assay (Figure 3.21D) was only possible because radical formation was slowed down due to limited access of a proton from the solvent to FAD-N5.
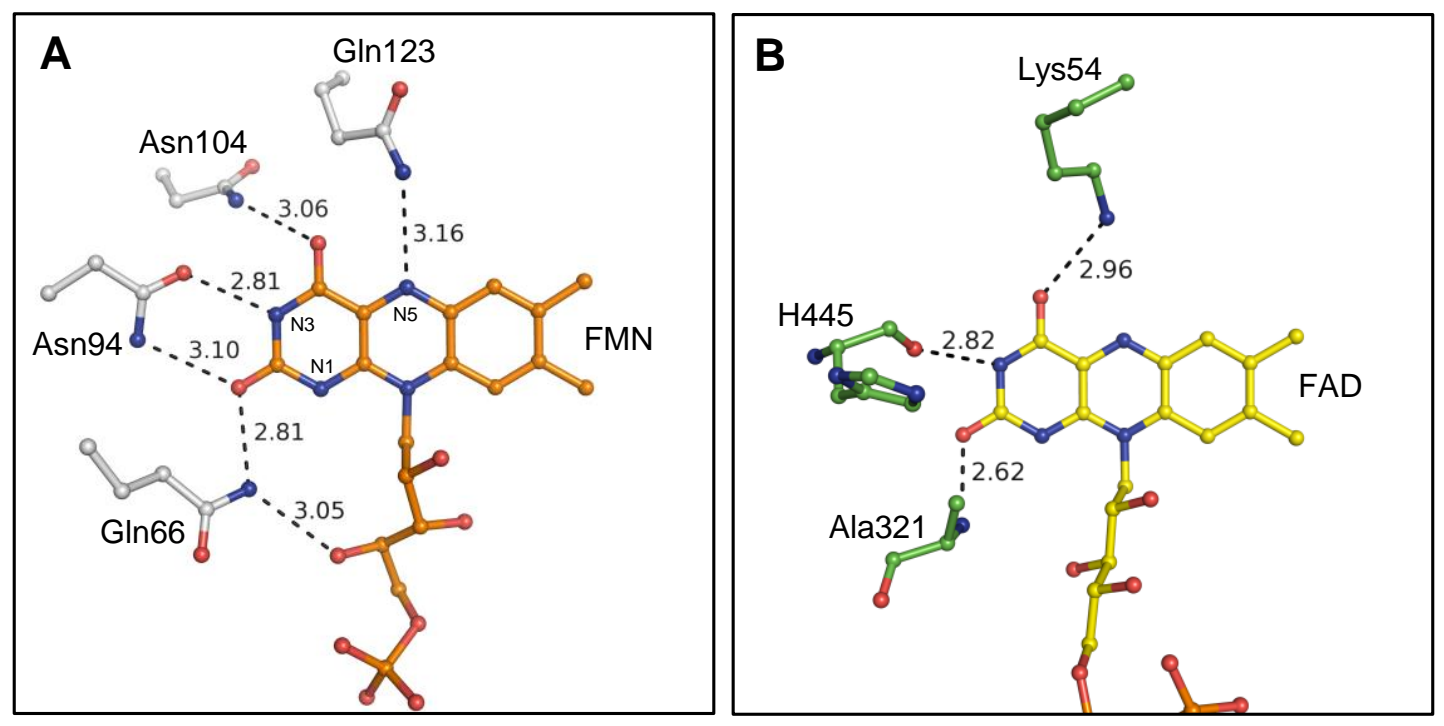

Figure 3.27 Comparison of the H-bonding network around the flavin cofactors in YF1 and $E c E 3$. (A)The HB network in LOV proteins consists of interactions between the $\mathrm{N} 5, \mathrm{C} 4=\mathrm{O}, \mathrm{N} 3 \mathrm{H}$, and $\mathrm{C} 2=\mathrm{O}$ atoms of the isoalloxazine moiety of FMN and a variety of conserved polar, uncharged amino acids(YF1; PDB: 4GCZ). In contrast, the interactions the isoalloxazine moiety in $E c E 3$ makes are limited to two main chain atoms and the conserved lysine residue (distances in $\AA$ ). 


\subsection{Expanding the Active Site - Glu354 is also a Mediator of the Flavin Redox Potential}

Mutation of Glu354, a second shell residue in EcE3 located at the dimer interface, to Lys354 reduced the sensitivity towards $\mathrm{NADH}$ inhibition of $E c \mathrm{E} 3$ and thereby affected the fermentation profile of E. coli (Kim et al. 2008). However, a more detailed or even a molecular investigation of this alteration was not conducted. In the following, the results obtained by Kim et al. will be summarized briefly and further extended with the help of spectroscopic and structural data. This extension shall broaden the understanding in which way the mutation of one single amino acid leads to such global effects.

Kim et al. purified EcE3E354K from strain SE2378 and determined kinetic steady-state parameters for both the native forward reaction using dihydrolipoic acid and the reverse reaction using NADH as reductant, respectively (Kim et al. 2008). Briefly, EcE3E354K only showed $50 \%$ activity in the native forward reaction, however the mutated enzyme was significantly more resistant towards NADH inhibition at saturation $\mathrm{NAD}^{+}$concentrations. In the reverse reaction, $E c \mathrm{E} 3 \mathrm{E} 354 \mathrm{~K}$ already reached approximately $75 \%$ of its maximum catalytic activity $(\sim 75 \mathrm{U})$ in the absence of added $\mathrm{NAD}^{+}$whereas the wild type enzyme required at least a $\mathrm{NAD}^{+} / \mathrm{NADH}$ ratio of 2.0 to reach its maximum $(\sim 70 \mathrm{U})$.

While the results obtained so far unambiguously demonstrate the physiological importance of the $\mathrm{NADH}$ sensitivity of $E c \mathrm{E} 3$ with the inherent potential of unexplored biotechnological applications, a molecular understanding of the altered enzyme properties is missing. To further elucidate the latter $E c \mathrm{E} 3 \mathrm{E} 354 \mathrm{~K}$ was derived from the wild type E. coli $\mathrm{E} 3$ enzyme by site-directed mutagenesis and subjected to both steady-state kinetics analysis and stopped-flow absorbance spectroscopy. The structural integrity was checked by far-UV CD spectroscopy revealing no significant alterations in the secondary structure compared to the wild type enzyme (5.6C). Further, the UV-Vis absorbance signature is congruent with the wild type spectrum indicating no influences of the mutation to the direct surrounding of the flavin cofactor $(5.2 \mathrm{~K})$. EcE3 follows a non-hyperbolic dependence with respect to lipoamide during the steady-state assay. However, EcE3E354K showed clear hyperbolic dependence leading to an $\sim 18.5$-fold increase in catalytic proficiency though the maximal specific activity deviated only by $\sim 10 \%$ (Figure 3.28A). The comparable catalytic activity of the reverse reaction and the altered behavior with respect to $\mathrm{NAD}^{+}$activation (Figure 3.28B) are consistent with the results obtained recently (Kim et al. 2008).

As described in 3.1.2 a 4-fold molar excess of NADH is sufficient to overreduce $E c E 3$ to the catalytic incompetent four-electron reduced state $\left(\mathrm{EH}_{4}\right)$. Assayed under the identical conditions $E c \mathrm{E} 3 \mathrm{E} 354 \mathrm{~K}$ resides in the catalytic active two-electron reduced state $\left(\mathrm{EH}_{2}\right)$ (Figure 3.28C). The presence of the charge transfer band at $523 \mathrm{~nm}$ and incomplete decrease in absorbance at $455 \mathrm{~nm}$ compared to the wild type $\mathrm{EH}_{4}$-level are indicative for the charge transfer complex between the thiolate formed by Cys50 and FAD. Thus, overreduction to the dead-end $\mathrm{EH}_{4}$ status is impaired in $E c \mathrm{E} 3 \mathrm{E} 354 \mathrm{~K}$ under the 
conditions tested. It should be noted that the charge transfer complex is formed with comparable rates and amplitudes as in the wild type (E354K: $k_{o b s}=135 \pm 2$ versus wt: $143 \pm 3 \mathrm{~s}^{-1}$ ) (Figure 3.28D). In addition, reduction to $\mathrm{EH}_{4}$ in the wild type enzyme is completed after $\sim 1 \mathrm{~s}$, whereas approximately $90 \%$ of the $523 \mathrm{~nm}$ charge transfer signal is still present in $E c \mathrm{E} 3 \mathrm{E} 354 \mathrm{~K}$ in the same time scale.
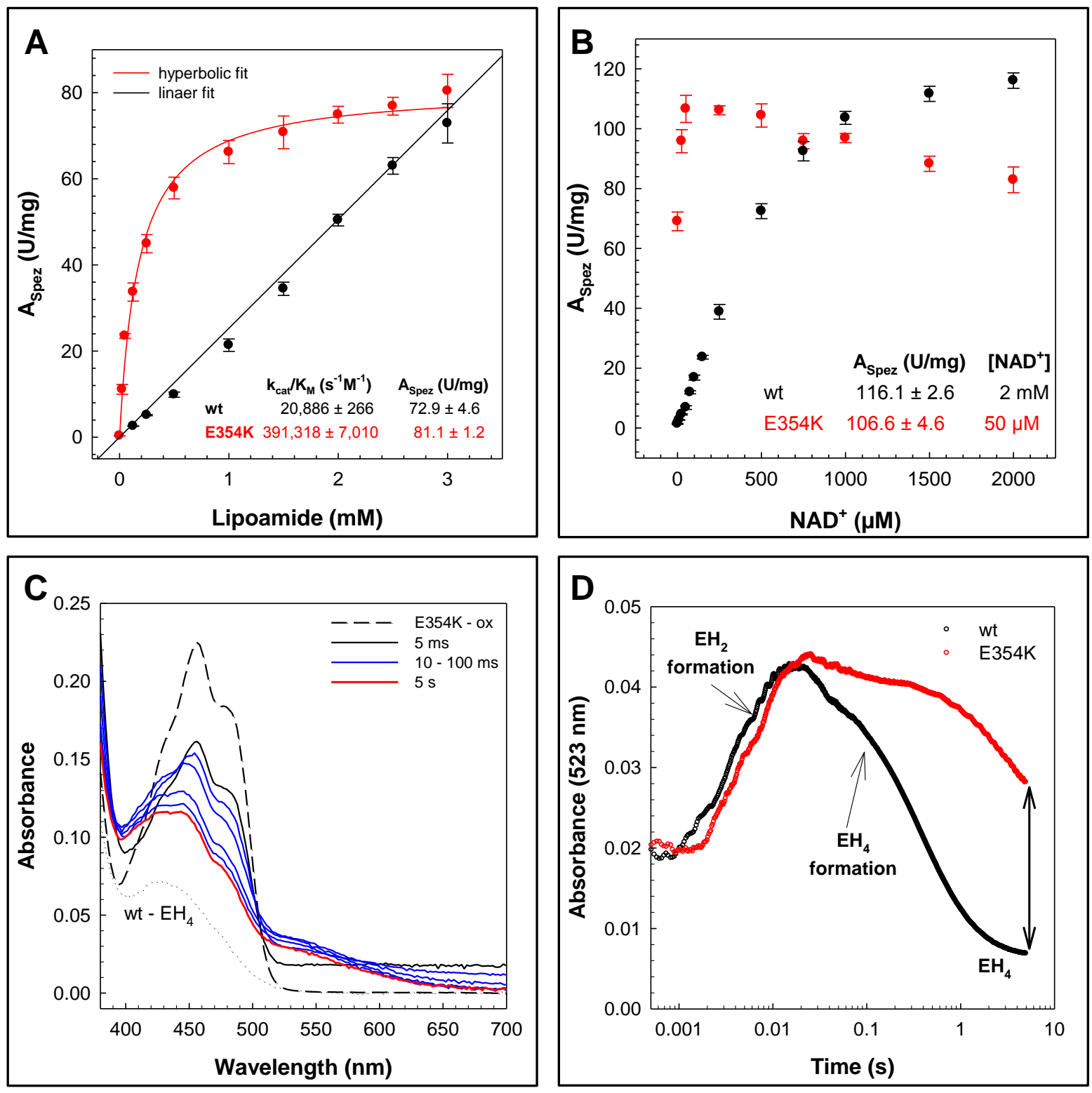

Figure 3.28 Kinetic and spectroscopic comparison between $E c \mathrm{E} 3 \mathrm{E} 354 \mathrm{~K}$ and $E c \mathrm{E} 3 \mathrm{wt}$.

A Comparison of the steady-state activity in dependence of lipoamide (2.2.4.1) and $\mathbf{B}$ of $\mathrm{NAD}^{+}$ (2.2.4.2). C Stopped-flow absorbance traces after mixing $20 \mu \mathrm{M} \mathrm{EcE3E354K} \mathrm{with} 100 \mu \mathrm{M} N A D H$ at $4{ }^{\circ} \mathrm{C}$. The dotted spectrum reflects the wild type $\mathrm{EH}_{4}$ spectrum as described in 3.1.2. D Comparison of stopped-flow absorbance traces at $523 \mathrm{~nm}$ under the conditions as described before. 
Considering the results obtained for mutations of Lys54 and Arg386 obtained by stopped-flow absorbance spectroscopy (3.3.2), Glu354 is also a ruler influencing the redox potentials of the different enzyme intermediate species. The altered redox potential in $E c \mathrm{E} 3 \mathrm{E} 354 \mathrm{~K}$ is evident since reduction with a 5-fold molar excess of $\mathrm{NADH}$ do not lead to $\mathrm{EH}_{4}$ formation. The spectroscopic signature obtained rather reflects a spectrum which is similar to the $\mathrm{EH}_{2}$ status described for pig heart lipoamide dehydrogenase under equal reduction conditions (Massey et al. 1960). The redox potentials of lipoamide dehydrogenases from different organisms have been investigated and the corresponding consequences for the catalytic properties have been drawn. WILKINSON AND WILLIAMS stated the bigger disproportionation of $\mathrm{EH}_{2}$ to $\mathrm{E}_{\mathrm{ox}}$ and $\mathrm{EH}_{4}$ in the E. coli lipoamide dehydrogenase to be the major difference compared to the pig heart enzyme (Wilkinson and Williams 1979, 1981). The reason is the larger separation of the $\mathrm{E}_{\mathrm{ox}} / \mathrm{EH}_{2}$ and $\mathrm{EH}_{2} / \mathrm{EH}_{4}$ redox potentials determined for the pig heart enzyme $(\sim 66 \mathrm{mV})$ compared to the closer separation $(\sim 53 \mathrm{mV})$ in the E. coli enzyme explaining the easier reduction to the 4-electron-reduced inactive enzyme. EcE3E354K therefore reflects a pig heart lipoamide dehydrogenase with respect to resistance against overreduction by NADH. To further underpin this assumption the absolute potentials of the $\mathrm{E}_{\mathrm{ox}} / \mathrm{EH}_{2}$ and $\mathrm{EH}_{2} / \mathrm{EH}_{4}$ redox pairs in the mutant enzyme should be determined as described earlier (Matthews and Williams 1976).

Though a direct investigation of the absolute potentials is missing, a further elucidation of the molecular basis for the altered catalytic properties of the mutated enzyme based on the secondary structure information of $E c E 3$ (3.1.3) was used. Glu354 is localized at the dimer interface directly above the interface domain within a loop connecting two anti-parallel $\beta$-strands (Figure 3.29). The distance between the carboxylic group of Glu354 and N5 of the flavin cofactor is approximately $15 \AA$ in the wild type enzyme. Thus, a direct interaction in the mutated enzyme is impossible. Further, there is no direct interaction with Lys54, Glu189 and Arg386 residues which were shown to modulate the flavin redox potential and hence susceptibility to overreduction by NADH (3.3.2). In addition, mutations of the latter residues showed remarkable effects in $E c E 3$ concerning affinity towards $\mathrm{NAD}^{+}$ (3.3.2). This indicates that the redox potential of the flavin cofactor is not only mediated by residues located in the direct vicinity (Lys54 and Glu189), but also by nearby active site residues (Arg386). A further, aspect which is thought to modulate the cofactor's redox potential is the subunit interaction of the two monomers forming the catalytic functional enzyme. Such ability was attributed to Tyr16 in the A. vinelandii $\mathrm{E} 3$ which forms a hydrogen bond with a histidine residue of the C-terminus of the adjacent monomer (Benen, Van Berkel, Veeger, et al. 1992). Mutation of the latter residue lead to a significantly raised redox potential, thus altering the catalytic function of the mutated enzyme. However, the corresponding residue in EcE3 (Tyr19) did not harbor a comparable mediator function (3.3.1.2). Taking into account that mutations at the dimer interface have the potential to act as determinate of the redox potential this role might also be attributed to Lys354 in the mutated E. coli enzyme. According to the position of Glu354 at the dimer interface, it is possible that a mutation to a lysine influences the subunit interaction yielding in a lowered redox potential of the FAD and hence a 
protection against overreduction by NADH. It still remains puzzling how this modulation proceeds and probably further mutations at the dimer interface are required to further elucidate that striking ability of a putative cross-talk between the two subunits.

In summary, the single mutation of Glu354 to Lys354 in E. coli lipoamide dehydrogenase not only led to a severe alteration of the regulation of the enzyme activity but thereby concomitantly changed the entire fermentation profile of the bacterium. Thus, the investigation of second shell residues with respect to their physiological role might have an unexplored potential for biotechnological applications.

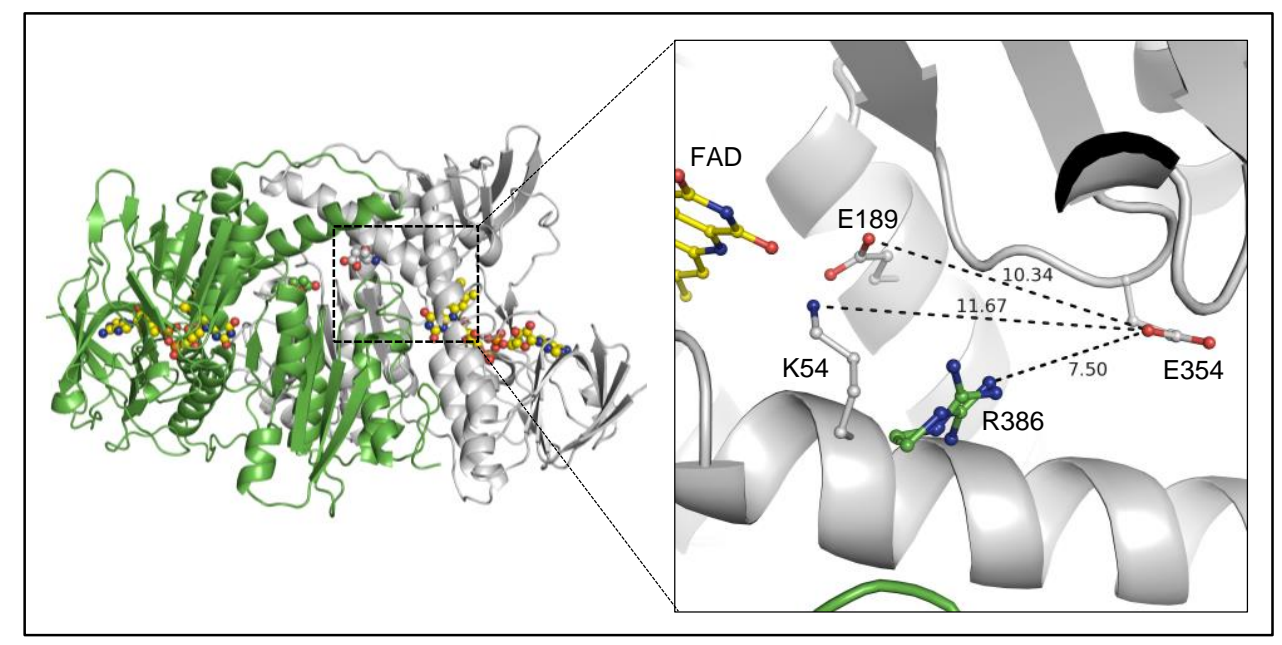

Figure 3.29 Position of Glu354 in $E c E 3$.

(left) Cartoon representation of the $E c \mathrm{E} 3$ homodimer. Glu354 and the FAD cofactor are shown in spheres. E354 is localized at the dimer interface, above the interface domain. (right) Distances ( $\mathrm{A}$ ) of Glu354 from selected active site residues involved in the oxidative half-reaction of $E c$ E3. 


\section{Summary}

The flavoenzyme lipoamide dehydrogenase is an ubiquitously distributed enzyme which is part of several multienzyme complexes involved in cellular carbon metabolism thus contributing to cellular homeostasis. It catalyzes the regeneration of reduced lipoamide cofactors using a disulfide exchange reaction of reduced lipoamide with a redox active cystine, transfer of two reducing equivalents from this disulfide bridge to the flavin cofactor and hydride transfer from the flavin site to final acceptor $\mathrm{NAD}^{+}$(Perham 2000; Reed 1974, 2001). Lipoamide dehydrogenases have been thoroughly studied mechanistically and structurally over the last decades. However, the studies so far lack detailed spectroscopic and structural information about the central FAD-C4a-cyteinyl adduct the enzyme forms during catalysis. Moreover, there are no structural information about the two-electron reduced state $\left(\mathrm{EH}_{2}\right)$. This doctoral thesis provides new insights into the nature of those intermediates that are transiently formed along the reaction coordinate in lipoamide dehydrogenase from $E$. coli by means of a structure-function analysis. By combining x-ray crystallography with spectroscopic methods, a more detailed picture of the $E c \mathrm{E} 3$ reaction cycle is now available helping to fill in the aforementioned blank.

Robust expression systems for both $E c \mathrm{E} 3$ and the innermost lipoyl domain (EcLip3) of the E2 component of $E c$ PDHc were established which allowed a convenient purification of both components to homogeneity. Several reaction intermediates could be identified and allocated in EcE3 with the help stopped-flow absorbance spectroscopy and a previously established model (Argyrou et al. 2002). However, reduction of the $E c \mathrm{E} 3$ with $\mathrm{NADH}$ (reverse direction) and, for the first time, with the reduced form of an isolated lipoyl domain $\left(E c \mathrm{Lip}_{3} \mathrm{H}_{2}\right.$; forward direction) did not allow the detection of an absorbance increase which is typical for the covalent flavin adduct, neither in single nor in sequential mixing experiments. These results underpin the transience of the intermediate and that it only accumulates to a very minor extend in solution making an observation difficult which is consistent with previous studies.

Though the spectroscopic detection of a covalent flavin adduct was not possible in the wild type enzyme there is evidence for an $\mathrm{NAD}^{+}$-induced FAD-C4a-cyteinyl formation in a monothiol variant, in which the interchange thiol forming the mixed disulfide during catalysis was substituted with an alanine. The necessity for the presence of $\mathrm{NAD}^{+}$to trigger adduct formation is presumably based on the following aspect: Binding of the positively charged nucleotide at the $r e$ face of the flavin increases the electrophilicity of the oxidized flavin and concomitantly permits an active site compression pivotal for adduct formation. However, the spectroscopic signatures clearly demonstrated that the covalent adduct only accumulated to a small amount in the monothiol variant as well.

The structural data obtained during this project suggest that the geometry of the FAD-C4a-cyteinyl adduct is most likely responsible for its transience and thus the reason for its limited accumulation in 
solution. Two X-ray structures of $E c E 3$ were solved, one in which $\mathrm{NAD}^{+}$was present during the phase of cryoprotection, and led to models with reasonable quality. Both structures highlight the local flexibility of the disulfide bridge contributing to the redox center and enabled the observation of structural rearrangements required for catalysis. The radiation-induced reduction of the disulfide bridge during data collection lead to partial opening of the latter and was thus used to trigger the formation of the covalent flavin adduct in crystallo. The EcE3 ground state structure allowed the observation of two different redox states of EcE3 simultaneously: the oxidized $\left(\mathrm{E}_{\mathrm{ox}}\right)$ and the twoelectron reduced state $\left(\mathrm{EH}_{2}\right)$. In addition, in the $\mathrm{E} 3-\mathrm{NAD}^{+}$structure electron density is observable that is consistent with an FAD-C4a-cyteinyl adduct, a central long-sought-after intermediate, whose existence has been predicted but has never been proven experimentally in a wild type enzyme. Geometrical analysis revealed that the adduct deviates from tetrahedral geometry harboring an $\mathrm{C} \beta-\mathrm{S} \gamma-$ C4a angle of $>117^{\circ}$. It is therefore most likely, that the high transience of the adduct is based on the distorted geometry of the C4a-cysteinyl adduct. Presumably, this prevents an elongated stabilization of the intermediate as it can be observed for the same kind of adducts in LOV domain photo receptors.

In order to stabilize the FAD-C4a-cyteinyl adduct with the perspective of a further improved structural analysis, the metabolic $E c \mathrm{E} 3$ should be converted into a light-sensing LOV domain. The generated $E c$ E3-pseudoLOV minimal system was not capable of light-induced adduct formation, but provided new insights into the protection mechanism against photo degradation in LOV domains and the formation of new flavin derivatives upon enzyme-bound photo degradation. The data suggest that covalent flavin adduct formation in LOV domains not only serves as structural feature which permits signal transduction, but also provides protection against photodegradation of the cofactor. The isolated flavin derivatives derived from the EcE3-pseudoLOV protein unambiguously demonstrate that the enzyme surrounding influenced the photo degradation process. A light-induced double bond formation in an intact FAD molecule has thus far never been described. However, further analytical and photochemical investigations are required to elucidate the mechanism yielding this modification.

The study also demonstrates the susceptibility of the enzyme to alterations of the flavin redox potential. Active site residues could be identified which act as rulers in mediating the potential thereby ensuring a suitable potential which allows optimal catalysis. Even more impressive is the fact, that the redox potential can be influenced by mutations which are not in the direct vicinity of the cofactor. It turned out that especially mutations at the dimer interface drastically alter the redox potential, either raising (R386A) or lowering it (E354K). This not only altered the enzymatic behavior of $E c E 3$ alone, but moreover led to global effects which altered the entire fermentation profile of the bacterium (Kim et al. 2008). A further investigation of the dimer interface with respect to its ability to modulate the flavin redox potential is therefore mandatory regarding the physiological importance of the enzyme. 


\section{Appendix}

\subsection{Amino Acid Sequences}

\section{$\underline{\text { SUMO-EcLip3 }}$}

Number of amino acids: $184 \quad$ Molecular weight: $20 \mathrm{kDa} \quad$ Theoretical pI: 4.67

\begin{tabular}{|c|c|c|c|c|c|}
\hline 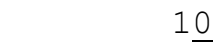 & $2 \underline{0}$ & $3 \underline{0}$ & $4 \underline{0}$ & $5 \underline{0}$ & 60 \\
\hline MSDSEVNQEA $\bar{A}$ & KPEVKPEVK $\bar{P}$ & ETHINLKVSD & GSSEIFFKI $\bar{K}$ & KTTPLRRLME & AFAKRQGKEM \\
\hline SLRFLYDG $\frac{70}{I}$ & $\begin{array}{r}80 \\
\text { RIQADQTPED }\end{array}$ & LDMEDNDII $\frac{90}{\mathrm{E}}$ & $\begin{array}{r}100 \\
\text { AHREQIGG } \mid \mathrm{AP}\end{array}$ & $\begin{array}{r}110 \\
\text { AAGVKEVNV }\end{array}$ & $\begin{array}{r}120 \\
\text { DIGGDEVEVT }\end{array}$ \\
\hline & 12 & 150 & 1 & 1 & $\underline{0}$ \\
\hline JMVKVGD & AAEQSLITVE & GDKASMEVPA & 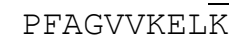 & VNVGDKVKTG & SLIMIFEVE \\
\hline
\end{tabular}

AAPA

$$
\underline{\perp}=\text { SUMO cleavage site }
$$

\section{$\underline{\text { EcLip3 }}$}

Number of amino acids: $86 \quad$ Molecular weight: 8777 Da Theoretical pI: 4.39

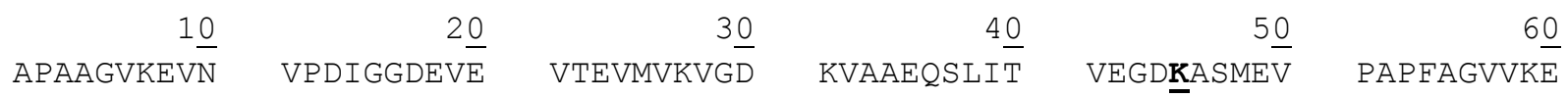

LKVNVGDKVK TGSLIMIFEV EGAAPA _ _ = lipoyl lysine

\section{$\underline{E c E 3 ~ d e r i v e d ~ f r o m ~ p G S 523 ~}$}

$\begin{array}{rrrrr}10 & 20 & 30 & 40 & 50\end{array}$

$\begin{array}{llllll}7 \underline{0} & 8 \underline{0} & 9 \underline{0} & 10 \underline{0} & 11 \underline{0} & 120\end{array}$

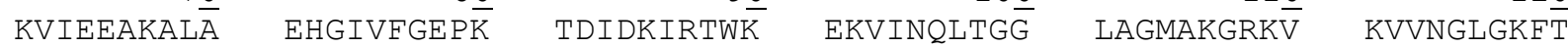

$\begin{array}{llllrr}130 & 140 & 150 & 160 & 170 & 180\end{array}$

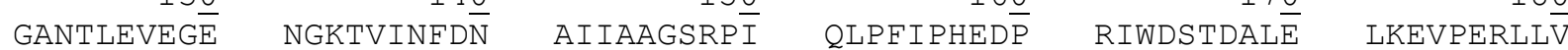

$19 \underline{0} \quad 20 \underline{0} \quad 21 \underline{0} \quad 22 \underline{0} \quad 23 \underline{0} \quad 24 \underline{0}$

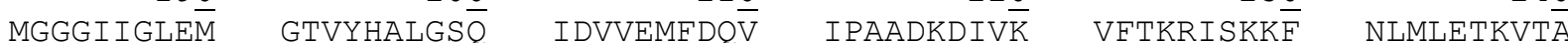

$25 \underline{0}-26 \underline{0} \quad 27 \underline{0} \quad 28 \underline{0} \quad 29 \underline{0}$

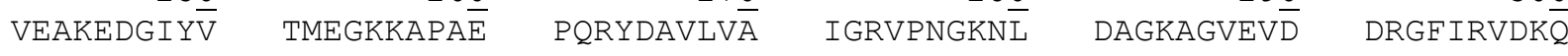

$\begin{array}{rrrrr}310 & 320 & 330 & 340 & 350\end{array}$ 


\begin{tabular}{|c|c|c|c|c|c|}
\hline $37 \underline{0}$ & 380 & 390 & 400 & $41 \underline{0}$ & 420 \\
\hline GLTEKEAKE $\bar{K}$ & GISYETATFP & WAASGRAIA $\bar{S}$ & DCADGMTKL $\bar{I}$ & FDKESHRVI $\bar{G}$ & GAIVGTNGGE \\
\hline & 440 & 450 & 460 & 470 & \\
\hline
\end{tabular}

\section{pET28a-EcE3}

10

MGSSHHHHHH

LGGVCLNVG $\frac{70}{\mathrm{C}}$

$13 \underline{0}$

LAGMAKGRKV

190

RIWDSTDALE

250

VFTKRISKK

310

DAGKAGVEVD

370

YFDPKVIPS $\bar{I}$

$43 \underline{0}$

FDKESHRVI $\bar{G}$

490

GSITDLPNPK AKKK
SSGLVPRIGS $\frac{20}{\mathrm{H}}$

MSTEIKTQVV

${ }^{40} \frac{0}{\mathrm{~S}}$

100

IPSKALLHVA

KVIEEAKALA $\stackrel{90}{\bar{A}}$

EHGIVFGEPK

$15 \underline{0}$

GANTLEVEGE

$16 \underline{0}$

NGKTVINFDN

210

MGGGIIGLEM

270

NLMLETKVTA

VEAKEDGIYV

330
LRTNVPHIFA

DRGFIRVDKO

IGDIVGQPML

$38 \underline{0}$

AYTEPEVAWV

390

GLTEKEAKEK

400

GISYETATFP

450

GAIVGTNGGE
$46 \underline{0}$

MGCDAEDIA
410

WAASGRAIA $\bar{S}$

470
TIHAHPTLHE

AAFRCADLGL

110

TDIDKIRTWK

170
AIIAAGSRPI

230

IDVVEMFDQV

290

PQRYDAVLVĀ

350

AHKGVHEGHV
ETVIVERYNT

EKVTNQI

180

QLPEIPHEDP

IPAADKDIVK

IGRVPNGKNI

AAEVIAGKKH

DCADGMTKLI

480

$\underline{1}=$ Thrombin cleavage site 


\subsection{UV-VIS Ground-state Spectra}

$\mathrm{UV}-\mathrm{Vis}$ ground-state spectra of $E c \mathrm{E} 3$ and variants thereof were recorded as described in 2.2.5.1. The insets show the differential spectra, calculated by subtraction of the wild type spectrum from the respective variant spectrum. Variants are ordered by the position of their mutation site starting from the N-terminus.
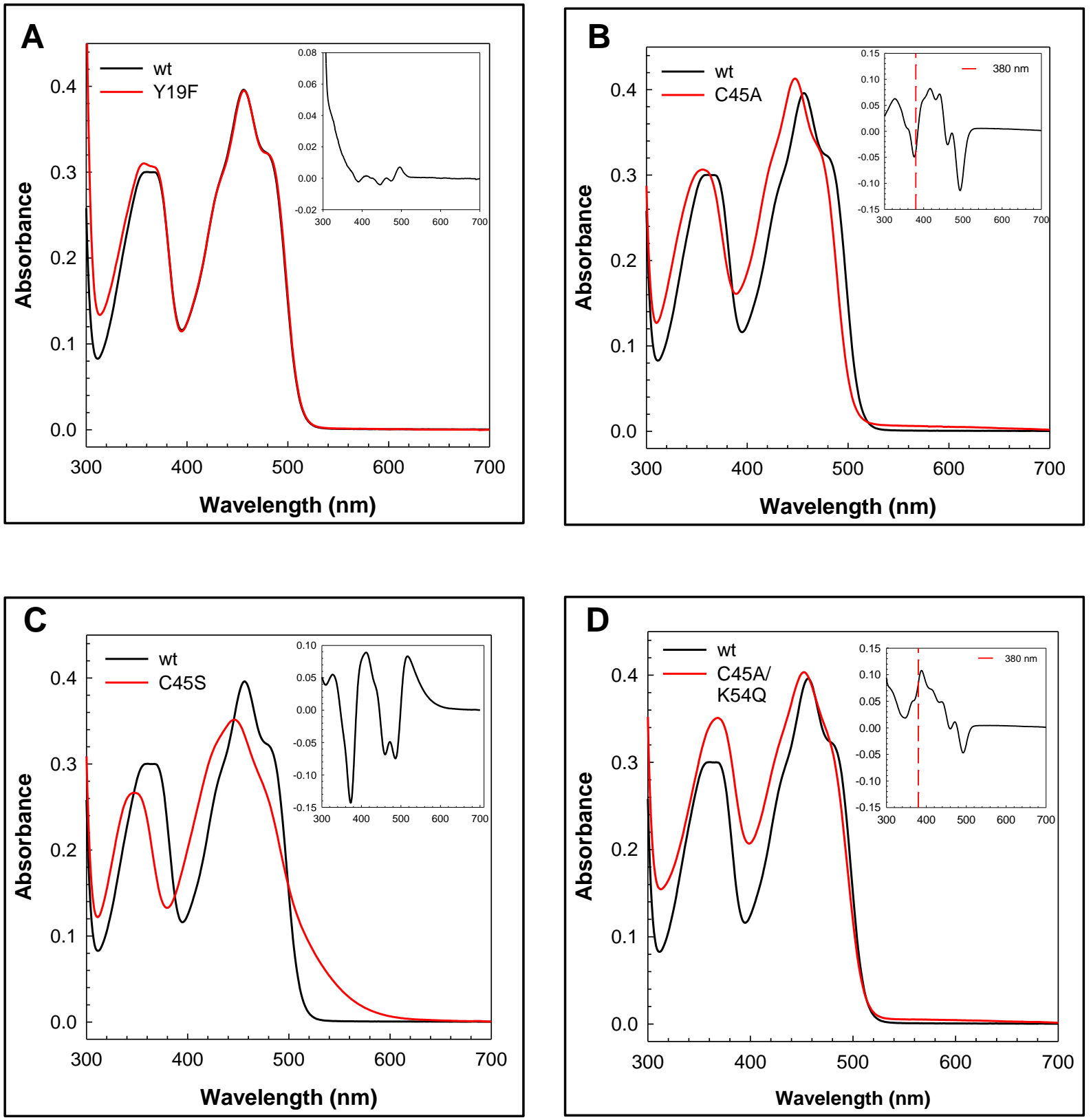

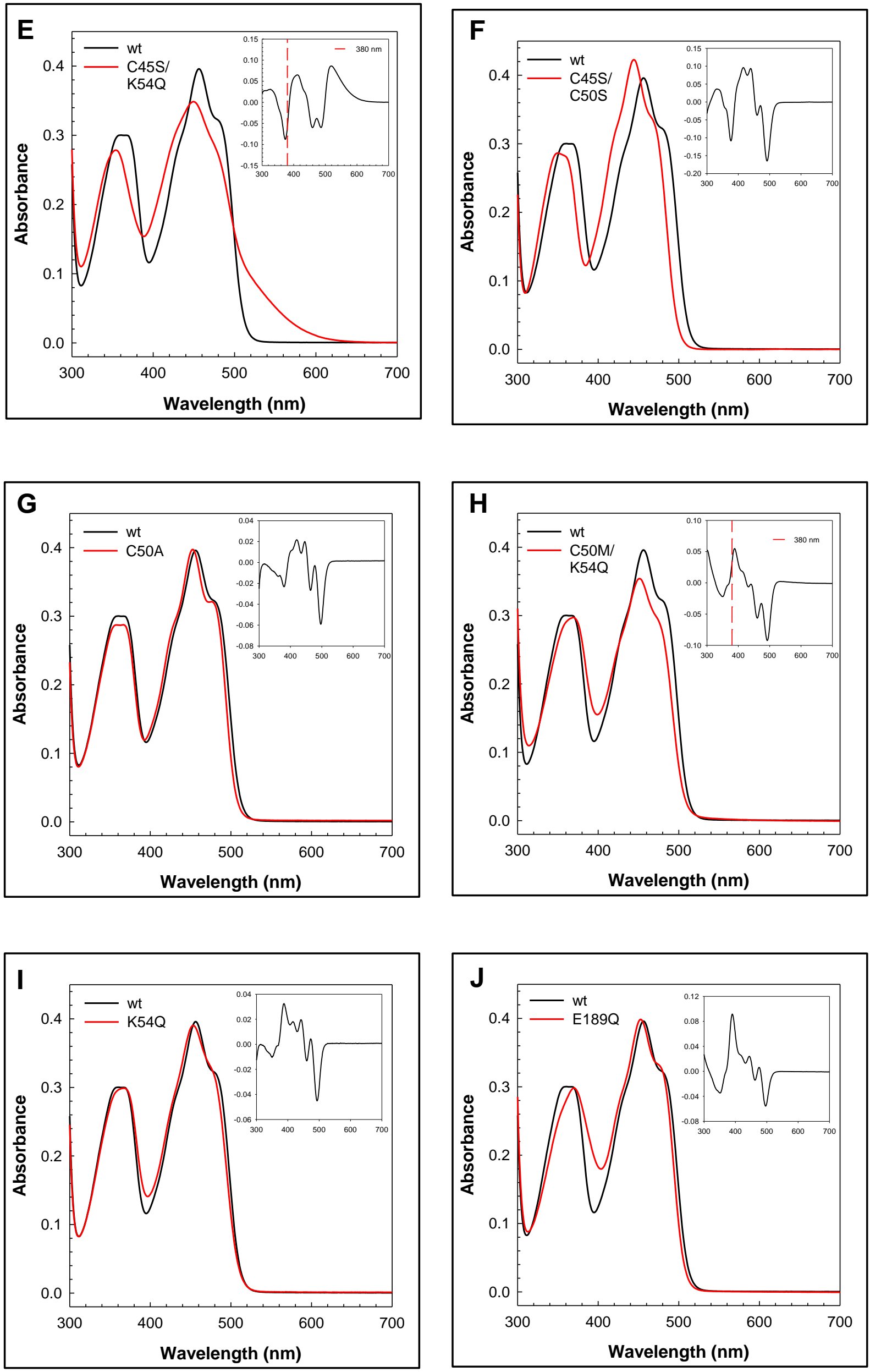

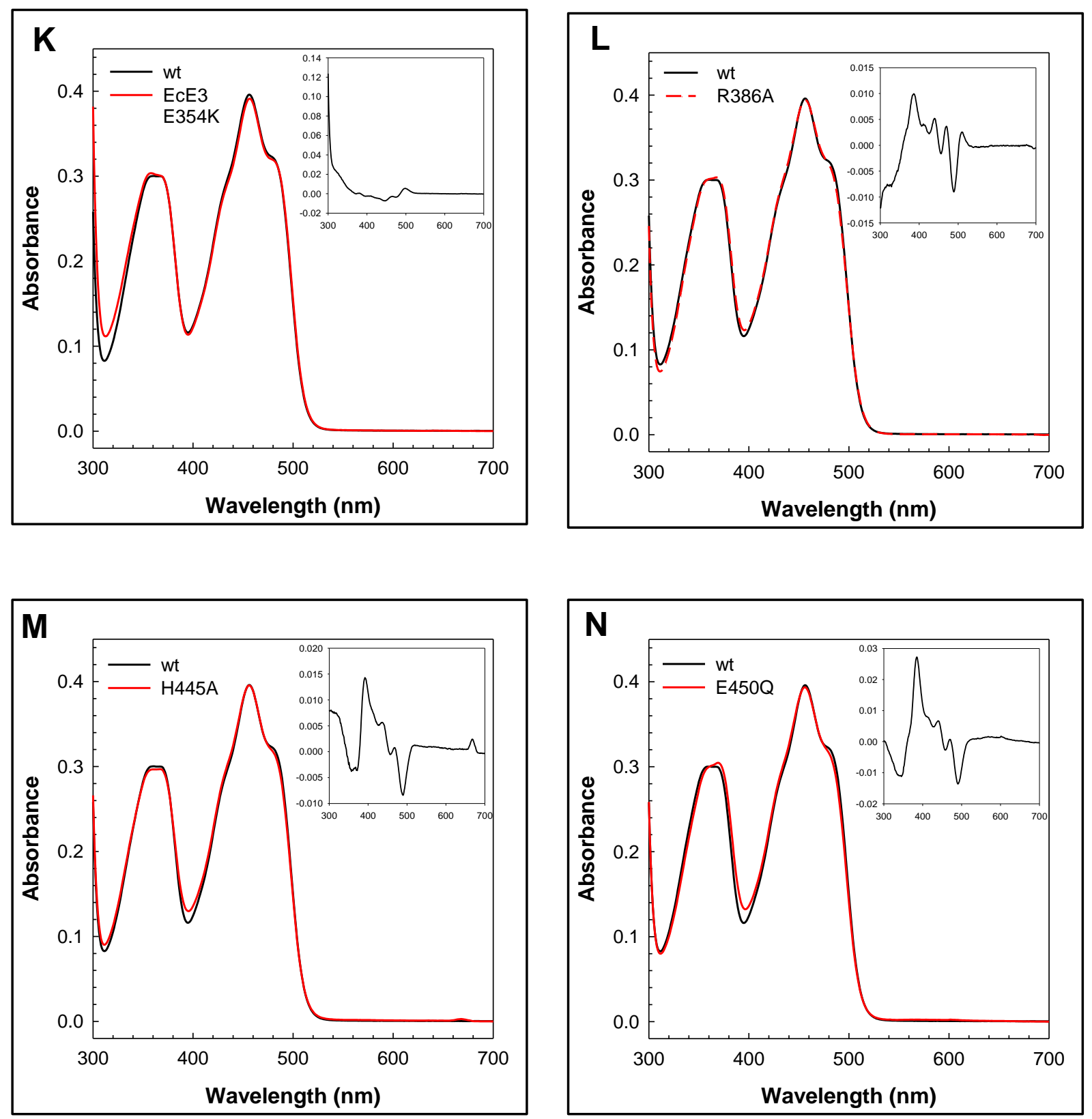


\subsection{Molar Extinction Coefficients}

Molar extinction coefficients were determined as described in 2.2.2.8. A linear regression was used for the calculations.
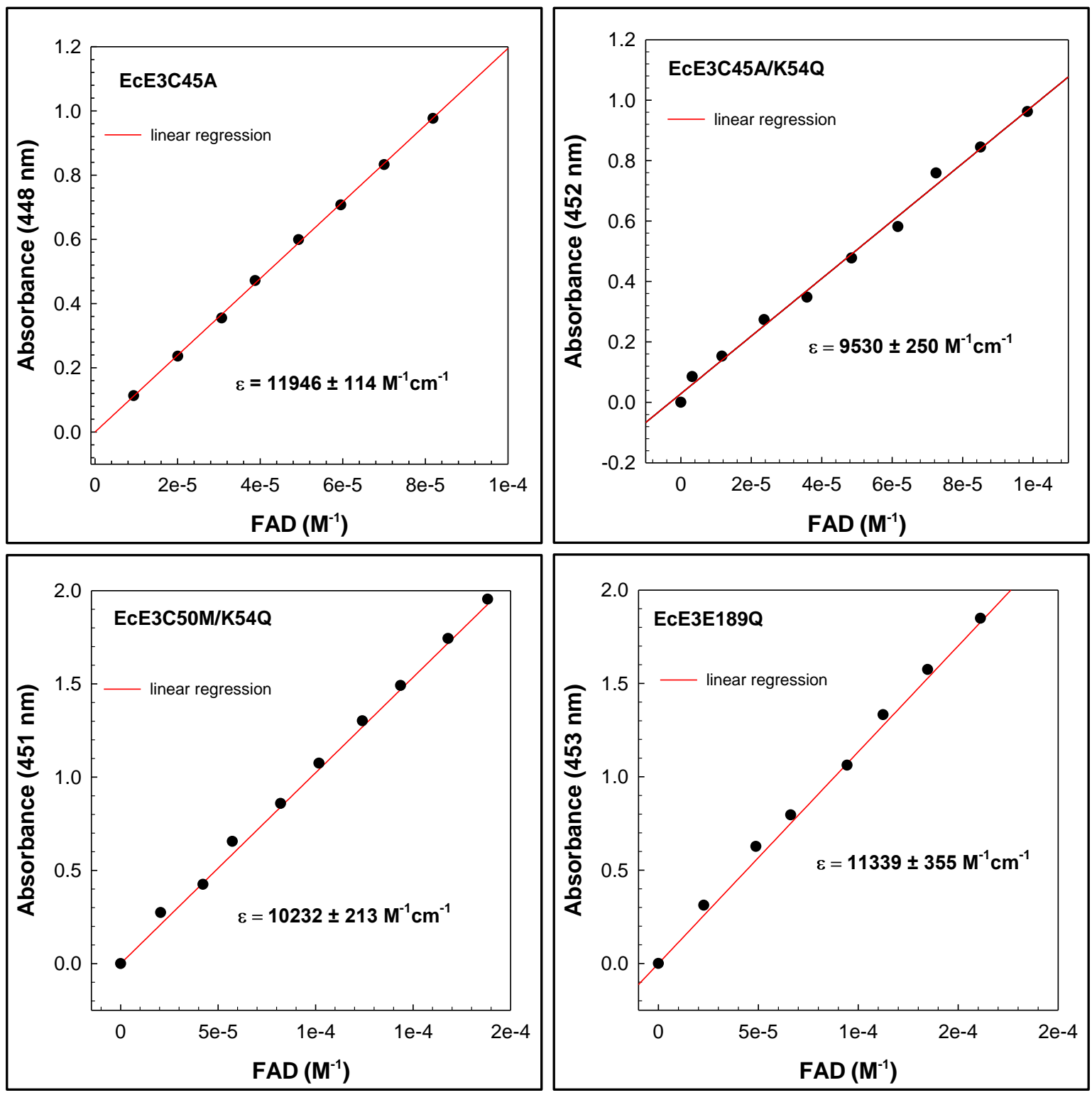


\subsection{Steady-state Activity of EcE3 and Variants thereof}

Steady-state activities in dependence of the lipoamide concentration of $E c \mathrm{E} 3$ and variants thereof were determined as described in 2.2.4.1. Data were fitted, if applicable, according to the Michaelis-Menten equation (red lines). Red arrows indicate NADH consumption at $0 \mathrm{mM}$ lipoamide. Variants are ordered by the position of their mutation site starting from the $\mathrm{N}$-terminus. The red arrows at a lipoamide concentration of $0 \mathrm{mM}$ indicate putative diaphorase activity when molecular oxygen serves as electron acceptor.
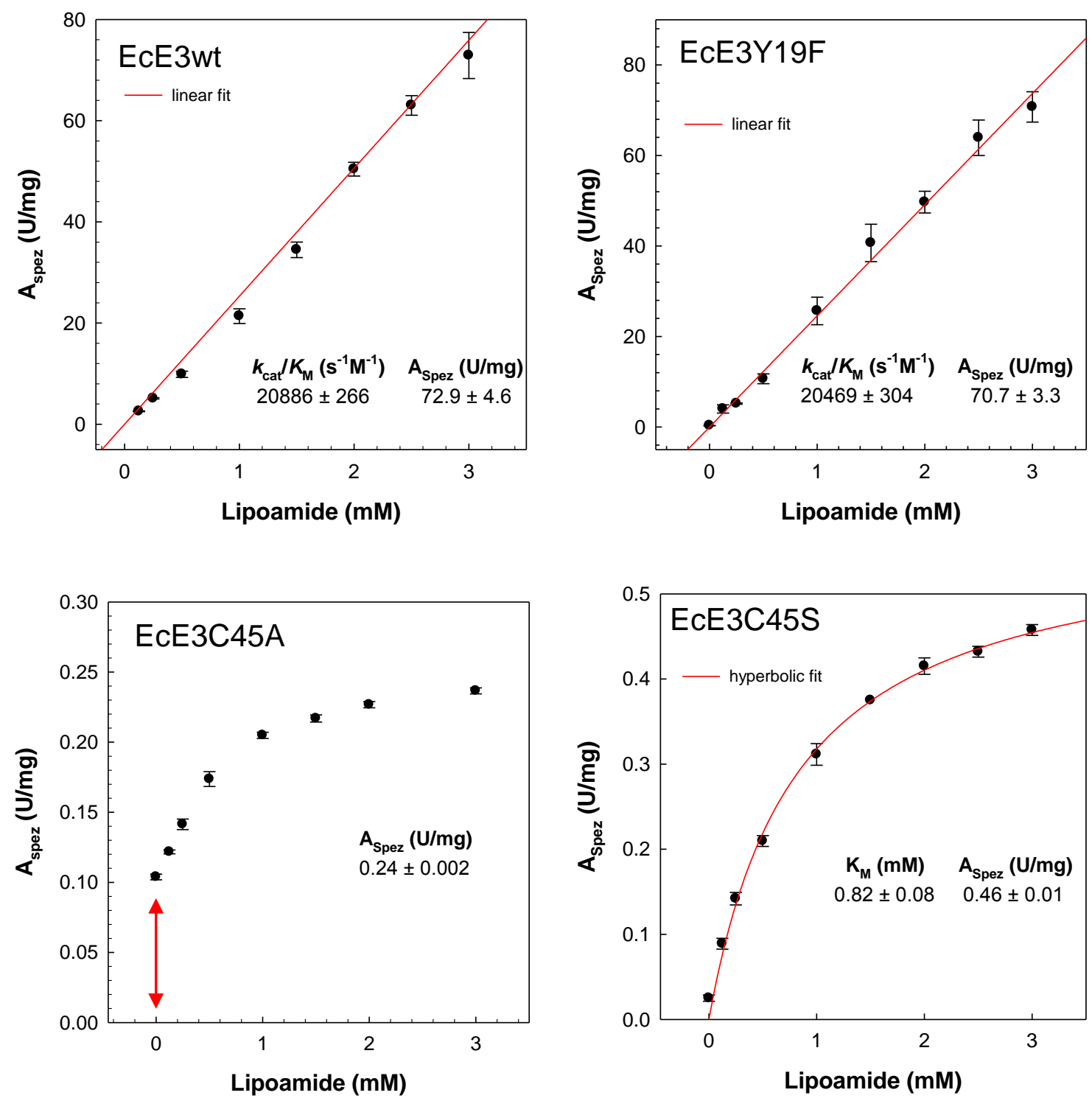

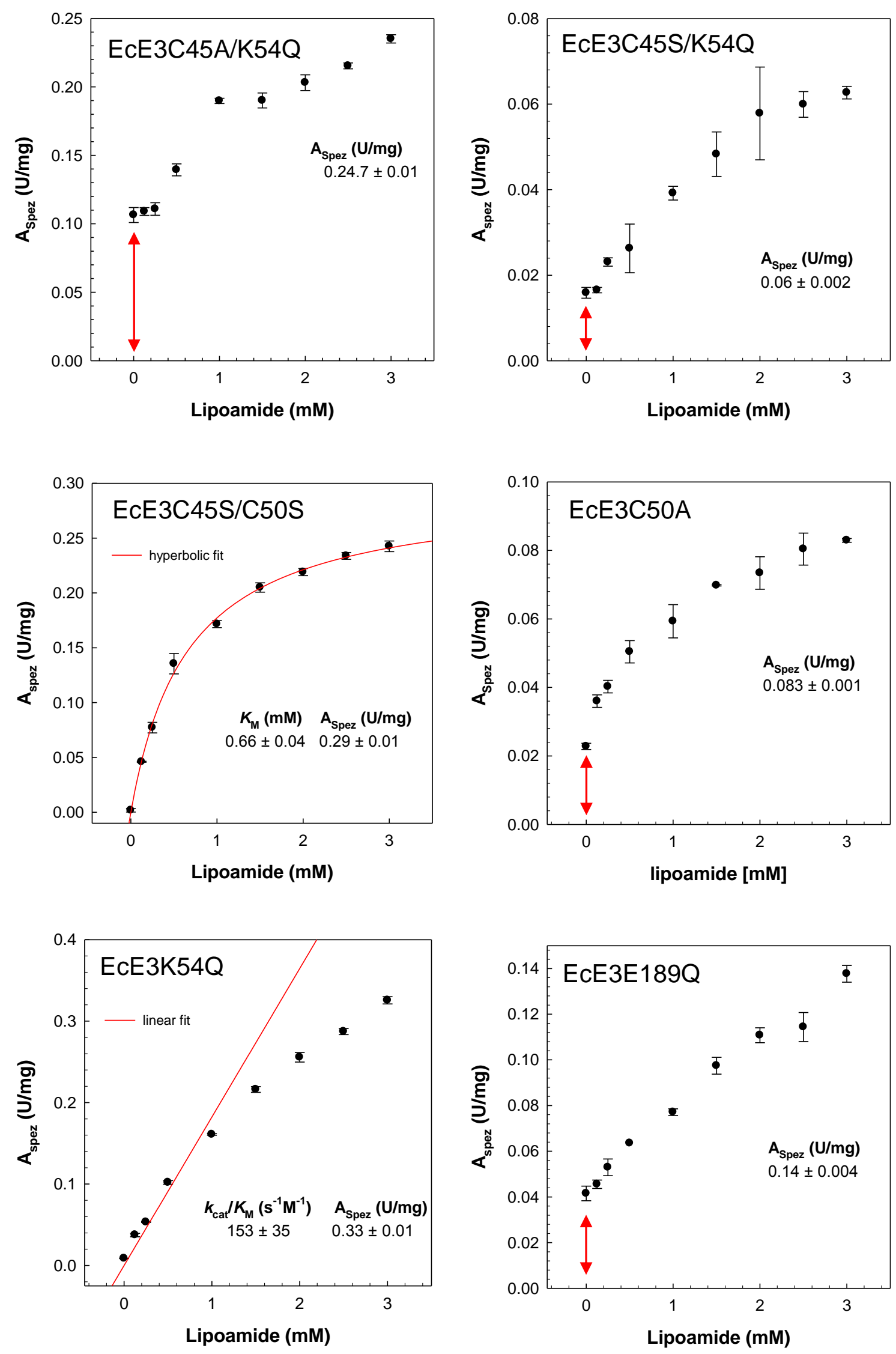

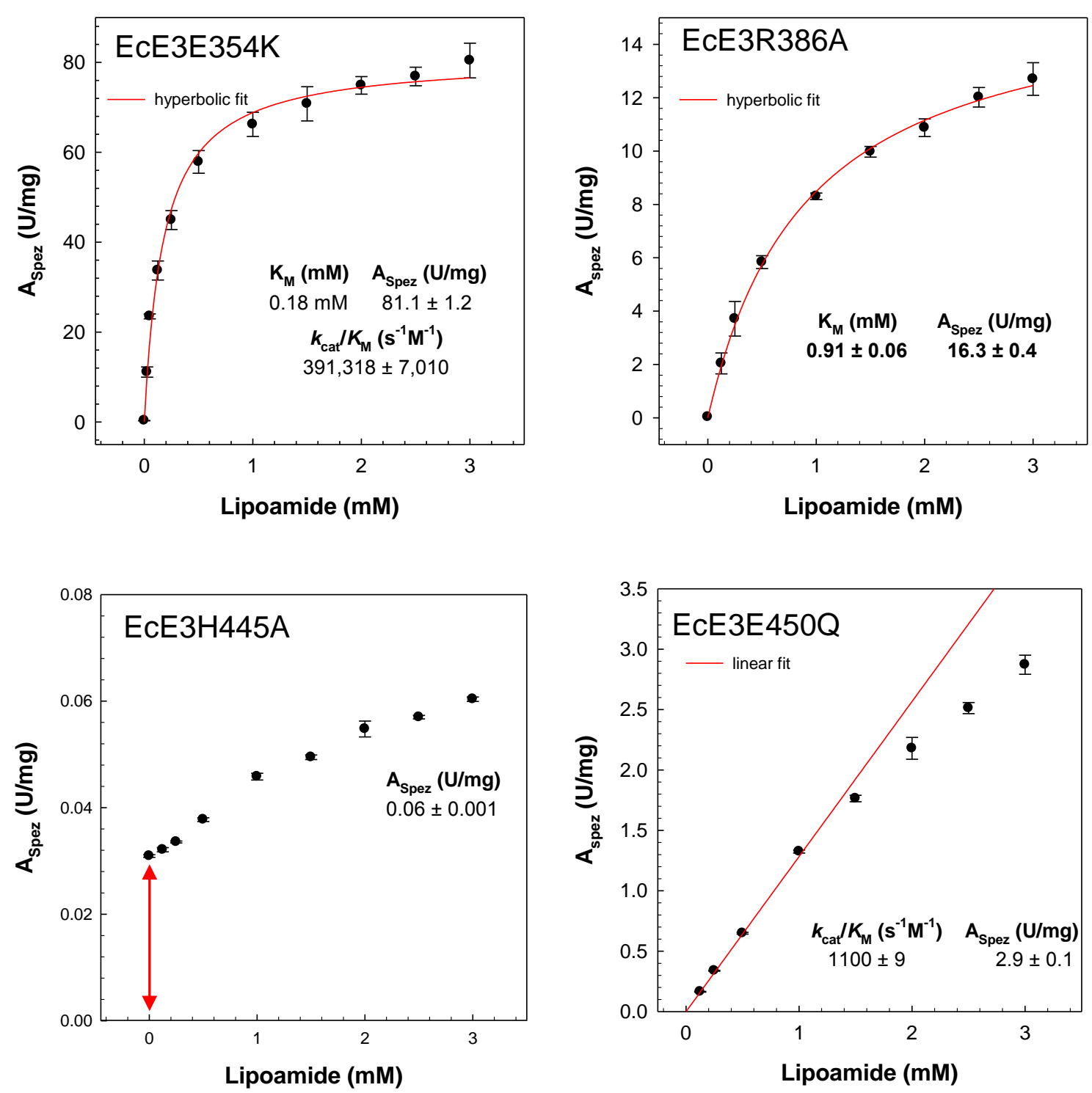


\subsection{Mechanistic Investigation of $E c \mathrm{E3}$}

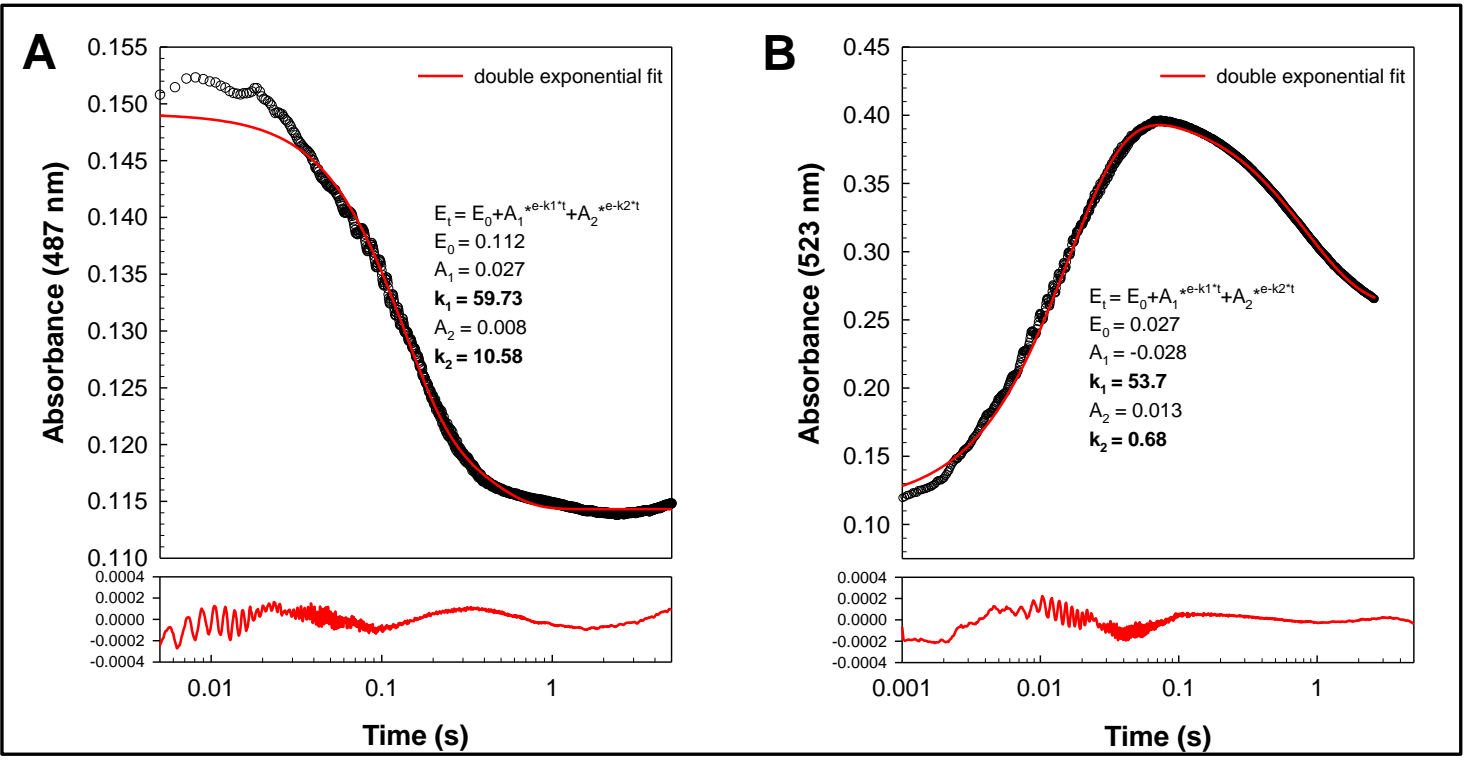

Figure 5.1 Reduction of $E c \mathrm{E} 3$ with NADH under single turnover conditions.

Stopped-flow absorbance traces after mixing $20 \mu \mathrm{M} \mathrm{EcE3} \mathrm{with} 20 \mu \mathrm{M}$ NADH at $487 \mathrm{~nm}$ (A) and at $523 \mathrm{~nm}(\mathbf{B})$ at $4{ }^{\circ} \mathrm{C}$ and at $\mathrm{pH}$ 7.6. The points are the experimental data, and the solid lines are the fits to a double exponential equation $\left(\mathrm{A}=\mathrm{A} 1 \cdot \mathrm{e}^{-\mathrm{k} 1 \cdot \mathrm{t}}+\mathrm{A} 2 \cdot \mathrm{e}^{-\mathrm{k} 2 \cdot \mathrm{t}}\right)$, respectively. Note the logarithmic time-scales. 

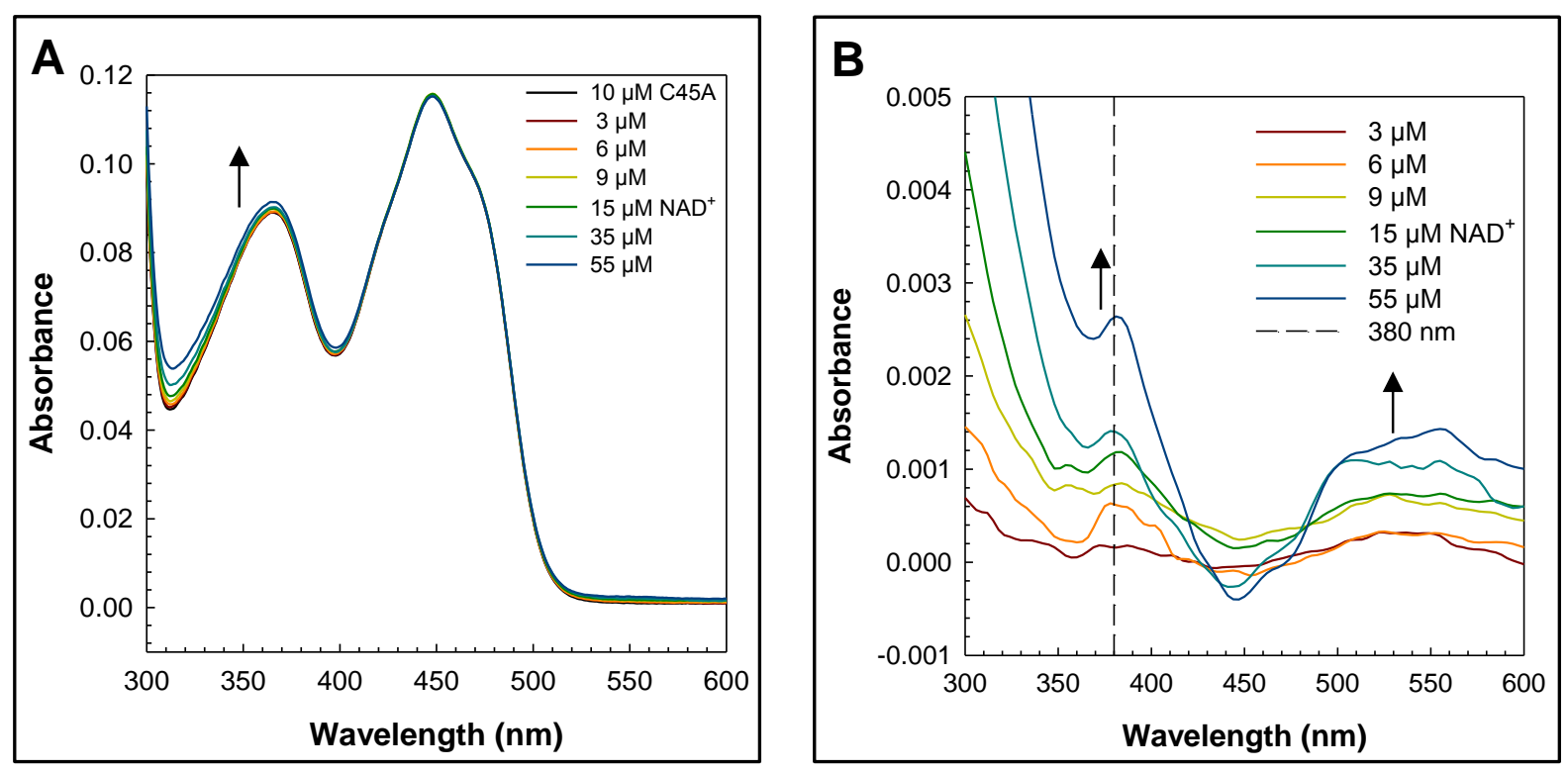

Figure 5.2 $\mathrm{NAD}^{+}$titration of $E c \mathrm{E} 3 \mathrm{EC} 45 \mathrm{~A}$.

(A) Lower temperature $\mathrm{NAD}^{+}$titration at $10{ }^{\circ} \mathrm{C}$ and in $100 \mathrm{mM}$ Tris $\mathrm{pH} 8.3$. Binding of $\mathrm{NAD}^{+}$does not significantly alter the spectrum. (B) Difference spectra of the same titration. NAD ${ }^{+}$induces a quenching of the flavin signature. Difference spectra were smoothed according to a bisquare weighting technique and a polynomial regression.
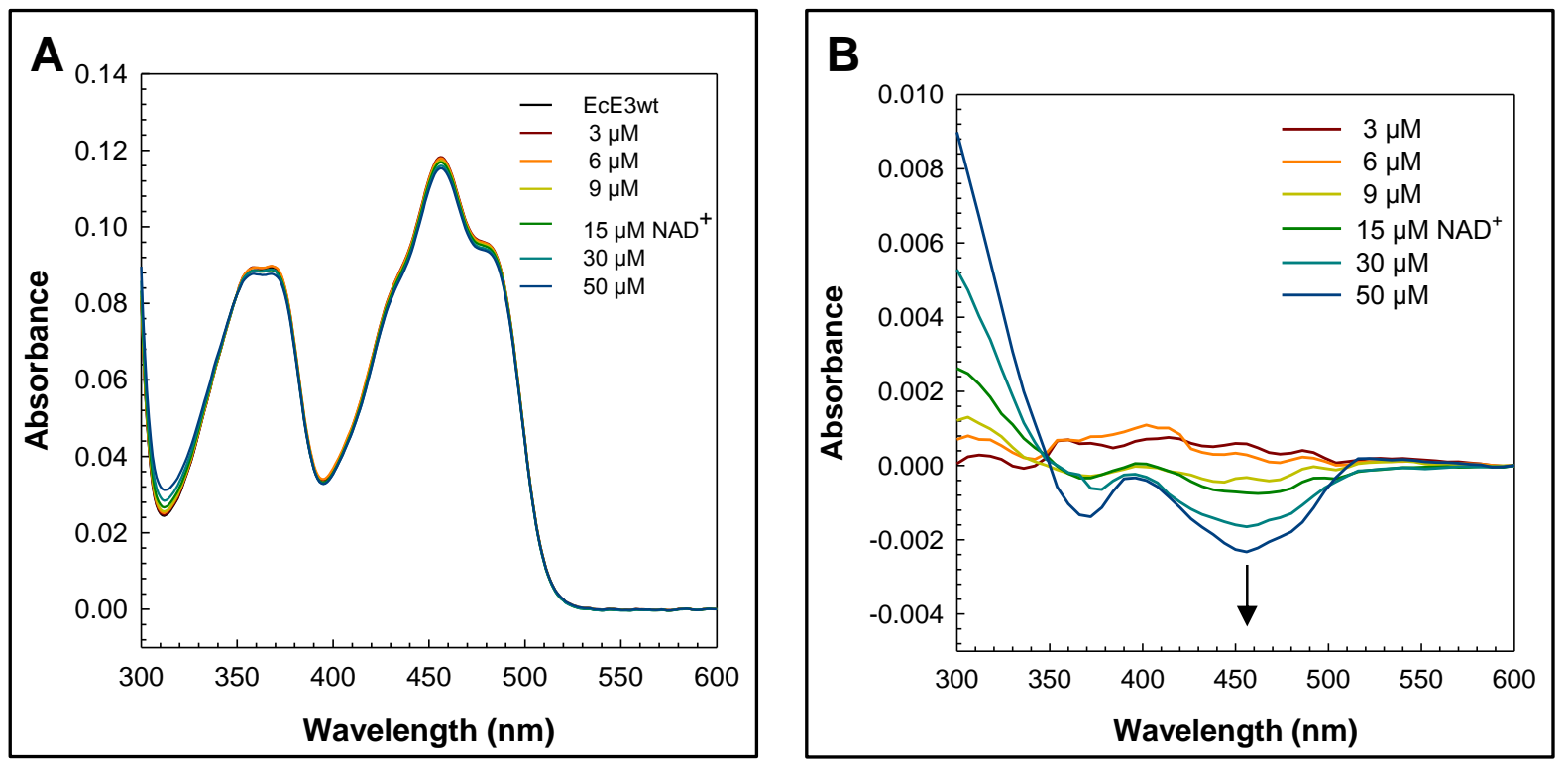

Figure 5.3 $\mathrm{NAD}^{+}$titration of $E c \mathrm{E} 3 \mathrm{wt}$.

(A) Lower temperature $\mathrm{NAD}^{+}$titration at $10{ }^{\circ} \mathrm{C}$ and in $100 \mathrm{mM}$ MES pH 6.0. Binding of $\mathrm{NAD}^{+}$does not significantly alter the spectrum. (B) Difference spectra of the same titration. NAD ${ }^{+}$induces a quenching of the flavin signature. Difference spectra were smoothed according to a bisquare weighting technique and a polynomial regression. 


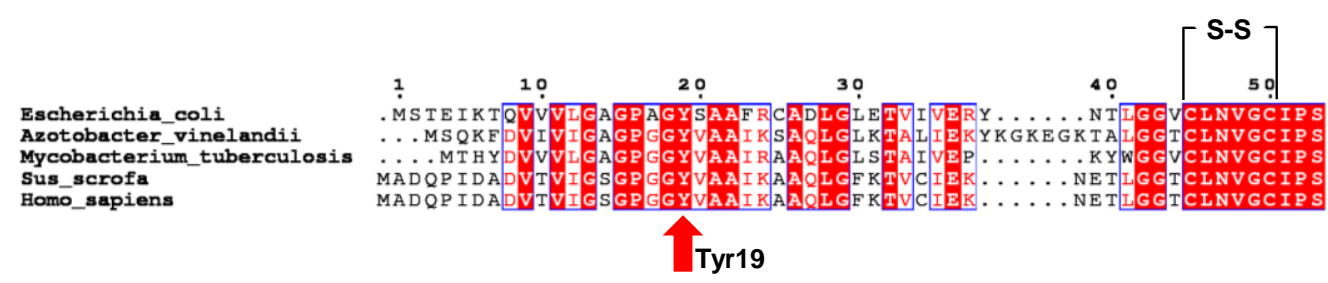

Escherichia_coli Azotobacter_vinelandii Mycobacterium_tuberculosis Sus_scrofa Homo_sapiens
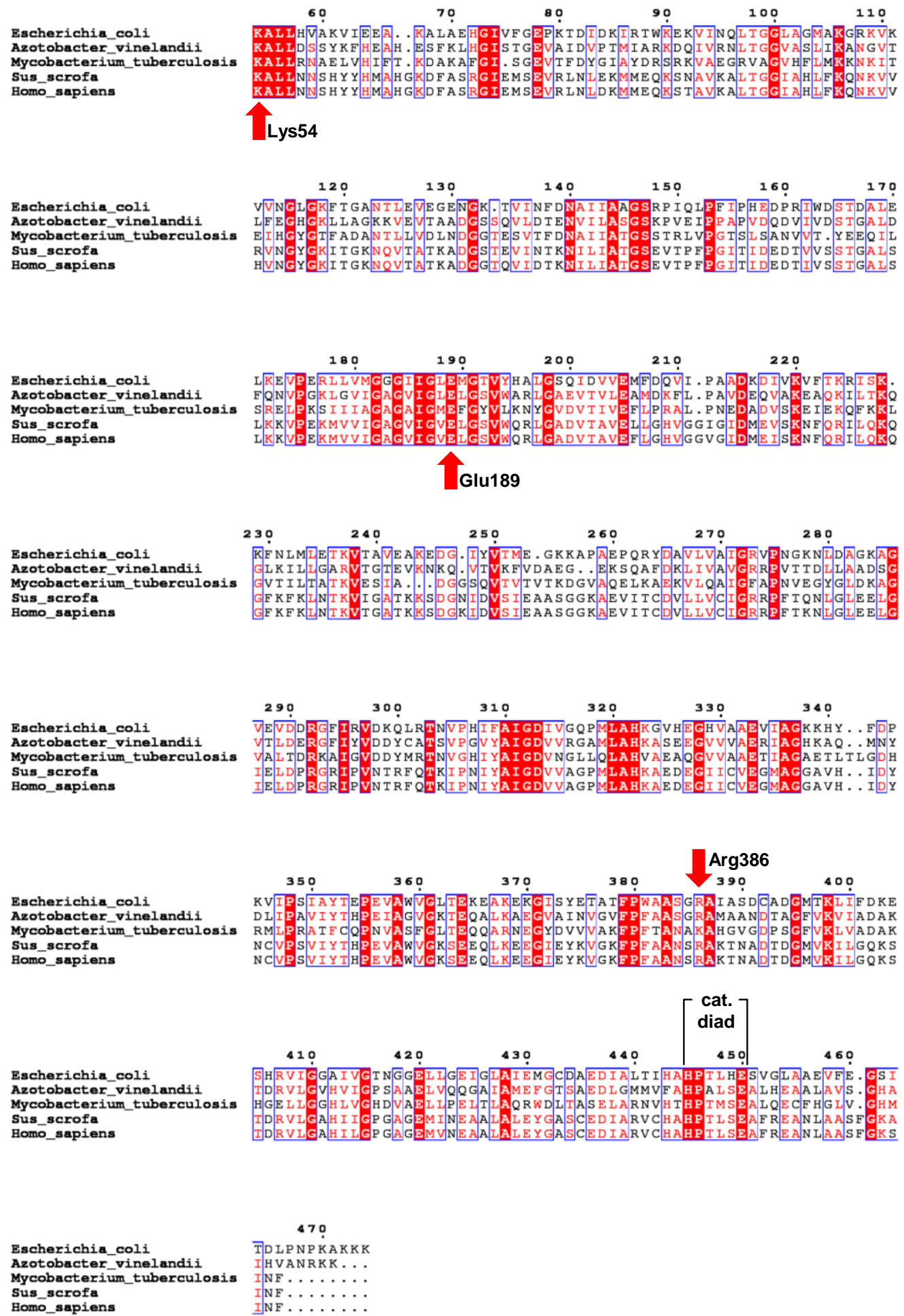

Figure 5.4 Primary amino acid sequences of E3 from different organisms.

The residues studied in this thesis are highlighted. Alignment was done with ClustalOmega. The figure was compiled with ESPript 3.0. 

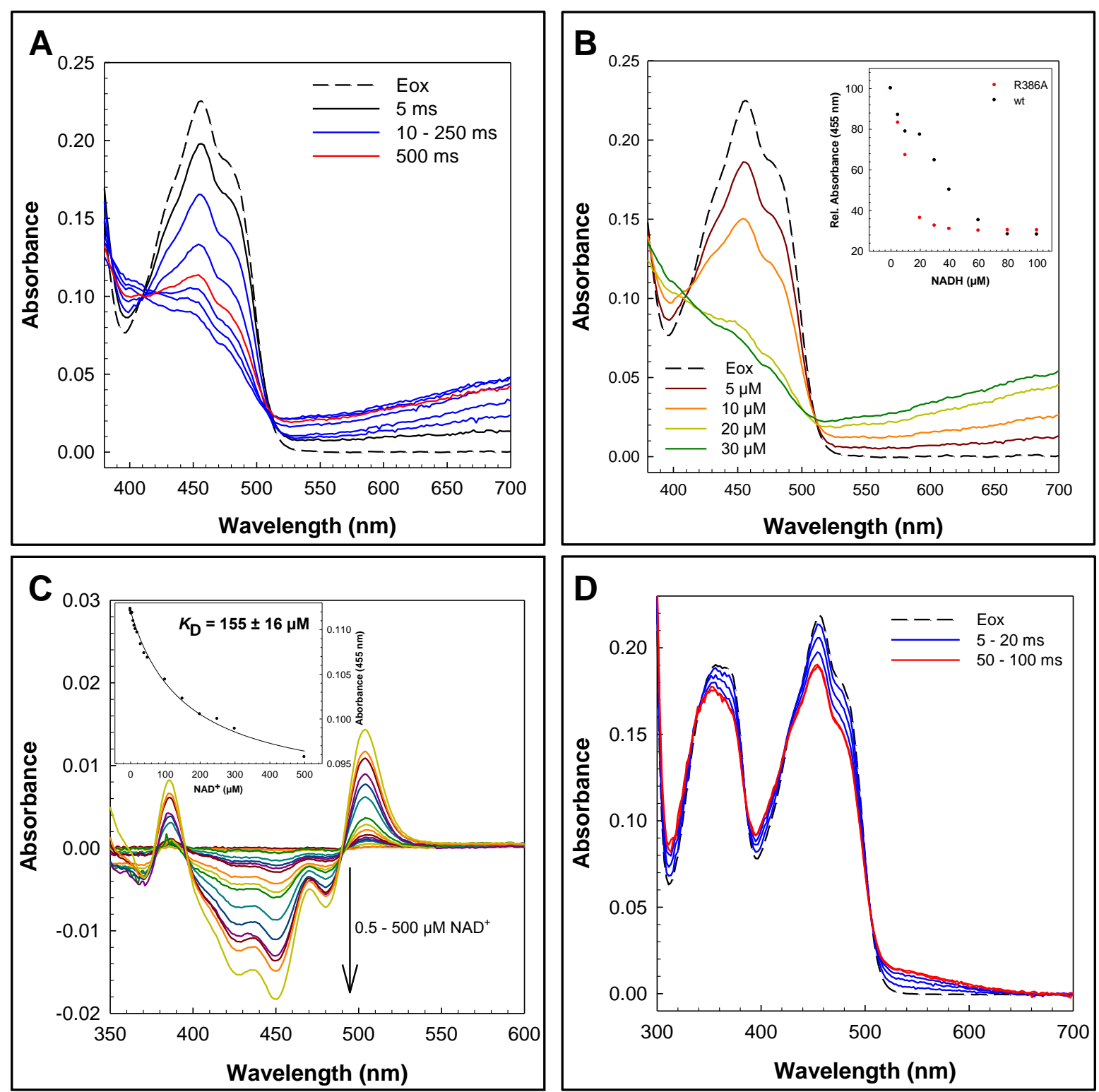

Figure 5.5 Spectral properties of $E c E 3 R 386 \mathrm{~A}$.

(A) Stopped-flow absorbance traces after mixing of $20 \mu \mathrm{M} \mathrm{EcE3R386A}$ with $100 \mu \mathrm{M}$ NADH at $4{ }^{\circ} \mathrm{C}$ and $\mathrm{pH}$ 7.6. The dashed line represents the oxidized form of the respective enzyme $\left(\mathrm{E}_{\mathrm{ox}}\right)$ derived from a single mixing event with buffer. The solid spectra were recorded using a diode-

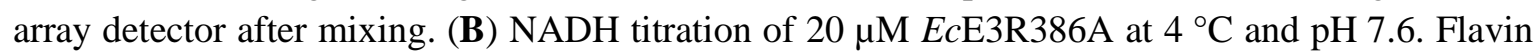
reduction is almost complete at an equimolar amount of NADH. The inset shows the relative absorbances at $455 \mathrm{~nm}$ plotted against the respective NADH concentration. (C) Lower temperature $\mathrm{NAD}^{+}$titration at $10{ }^{\circ} \mathrm{C}$ and $\mathrm{pH}$ 7.6. The inset shows a hyperbolic fit for decrease in absorbance at $455 \mathrm{~nm}$ giving an apparent binding constant of $\sim 155 \mu \mathrm{M}$ for the nucleotide. (D) Stopped-flow

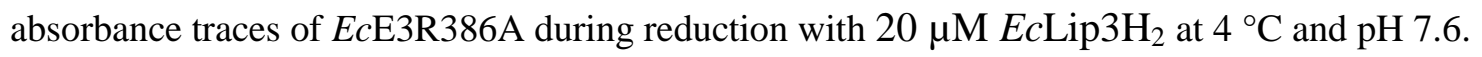



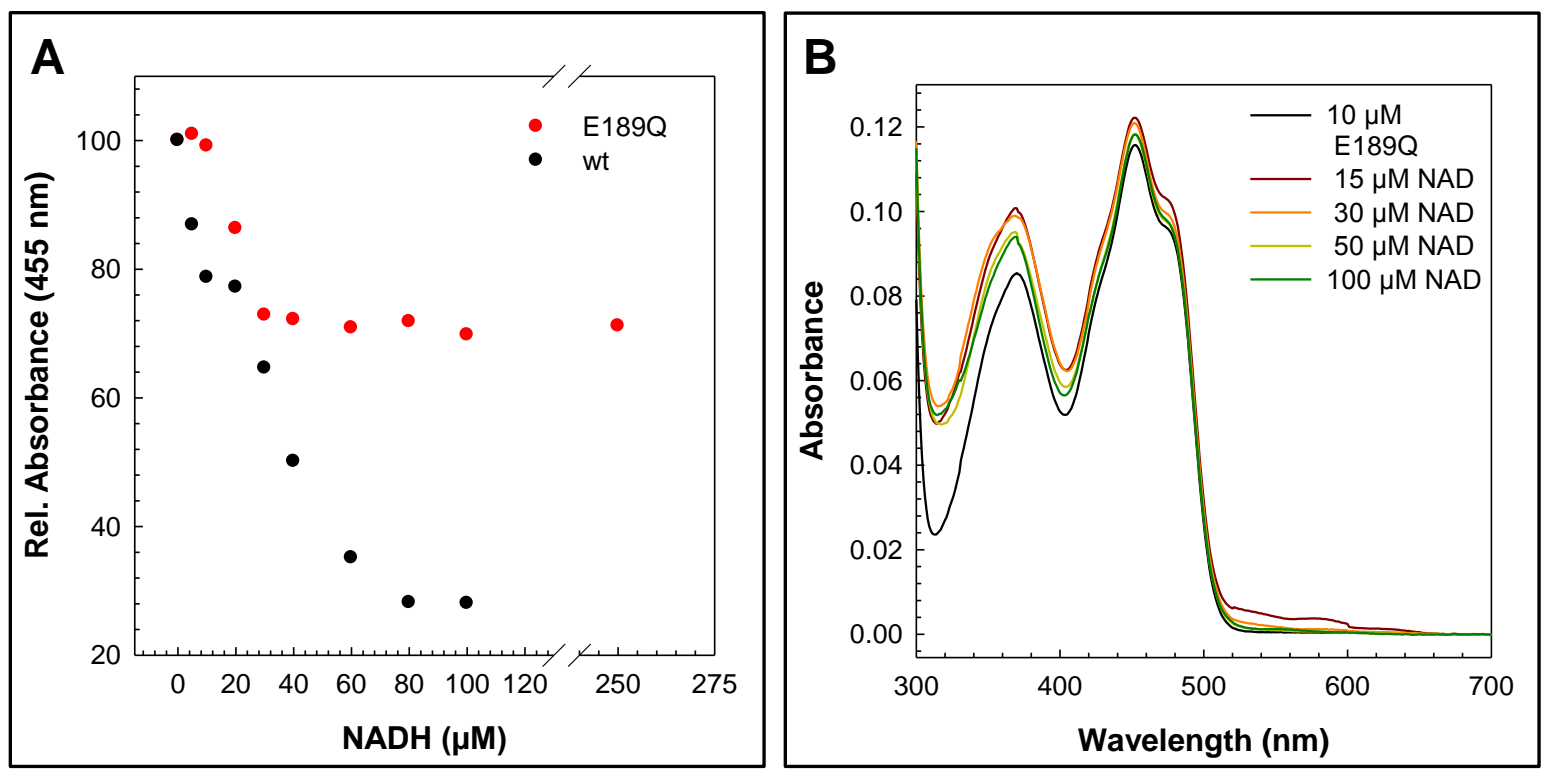

Figure 5.6 Further spectral properties of $E c \mathrm{E} 3 \mathrm{E} 189 \mathrm{Q}$.

(A) Relative absorbance at $455 \mathrm{~nm}$ derived from a stopped-flow NADH titration of EcE3E189Q at $4{ }^{\circ} \mathrm{C}$ and $\mathrm{pH}$ 7.6. No complete reduction of the flavin can be achieved with a 12.5 -fold molar excess of NADH in this variant. (B) Lower temperature $\mathrm{NAD}^{+}$titration (difference spectra) at $10{ }^{\circ} \mathrm{C}$ and $\mathrm{pH}$ $\mathrm{pH}$ 7.6. Binding of $\mathrm{NAD}^{+}$leads to an increase in absorbance at $360 \mathrm{~nm}$. 


\subsection{Far-UV CD Spectra}

Far-UV CD spectra of $E c \mathrm{E} 3$ and variants thereof were recorded as described in 2.2.5.2.
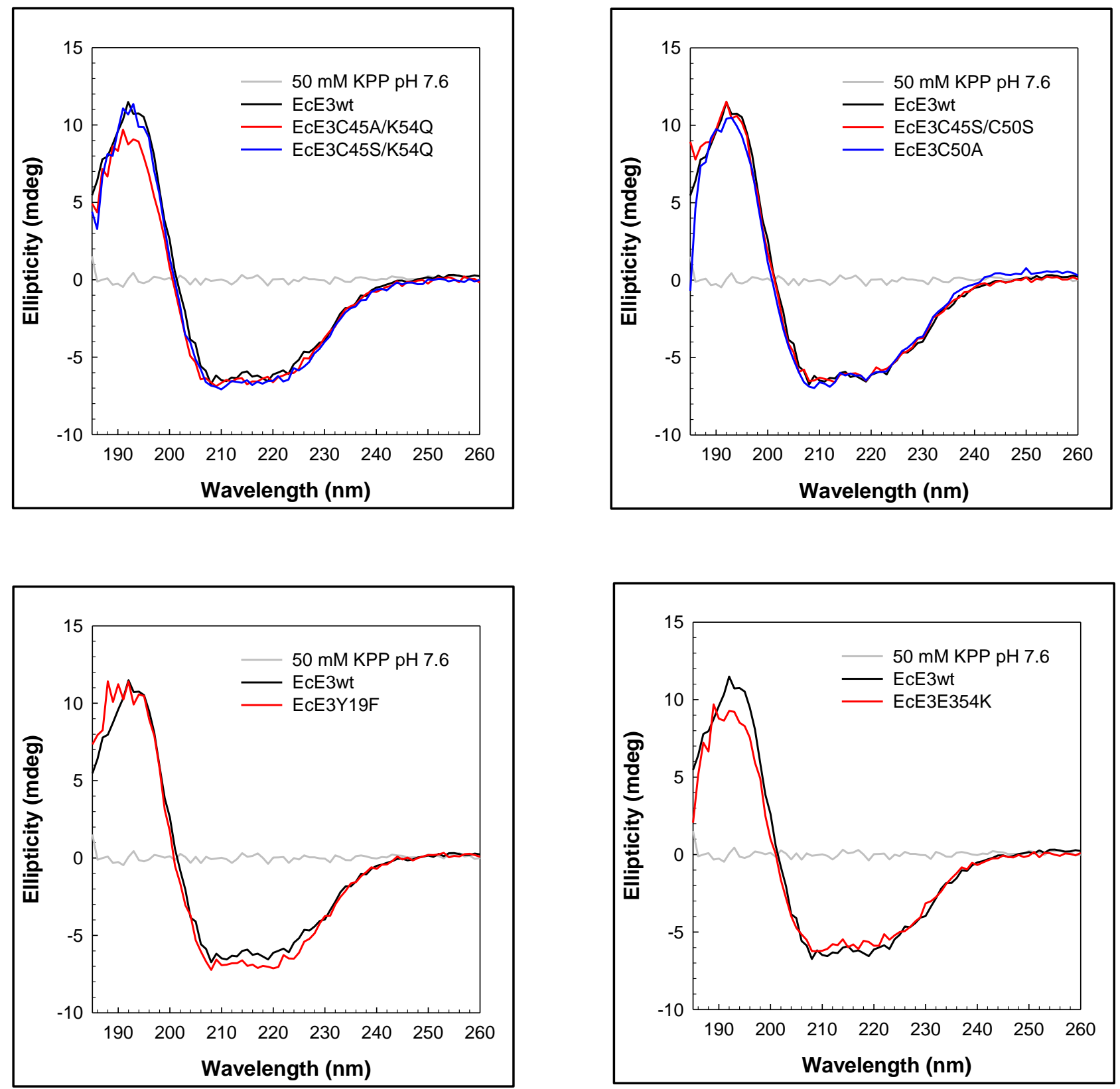


\subsection{Photobleaching of EcE3}

$E c \mathrm{E} 3$ variants were photobleached as described in 2.2.2.10.
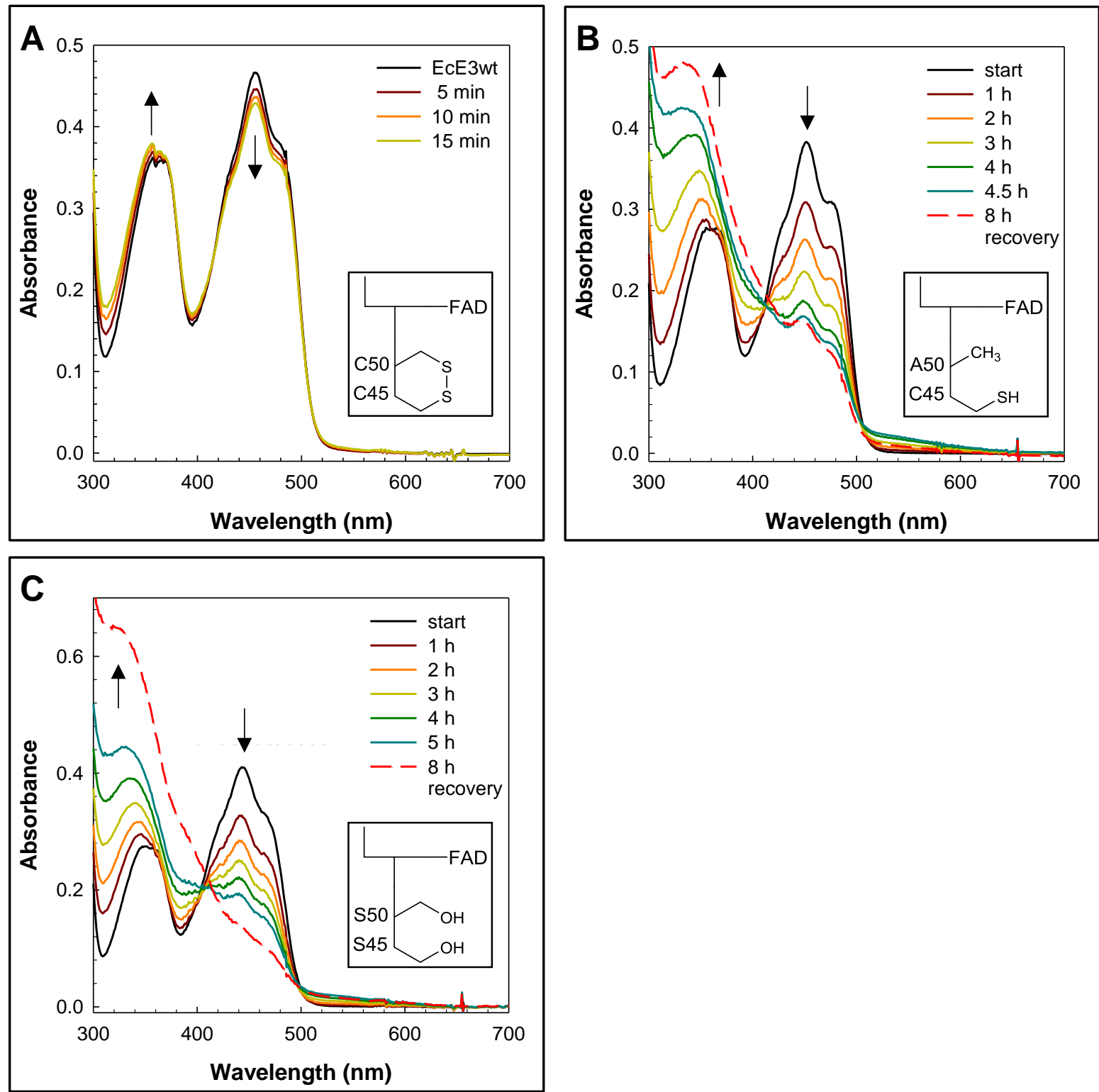

Figure 5.7 Photobleaching of wild type, monothiol or non-thiol variants of $E c E 3$.

Bleaching of EcE3wt (A), EcE3C50A (B) and of EcE3C45S/C50S (C). The insets show a highly simplified model of the respective active sites used for photobleaching control experiments. 


\subsection{EPR}

$E c \mathrm{E} 3 \mathrm{C} 45 \mathrm{~A} / \mathrm{K} 54 \mathrm{Q}$ (pseudoLOV) was subjected to EPR measurements as described in 2.2.5.4

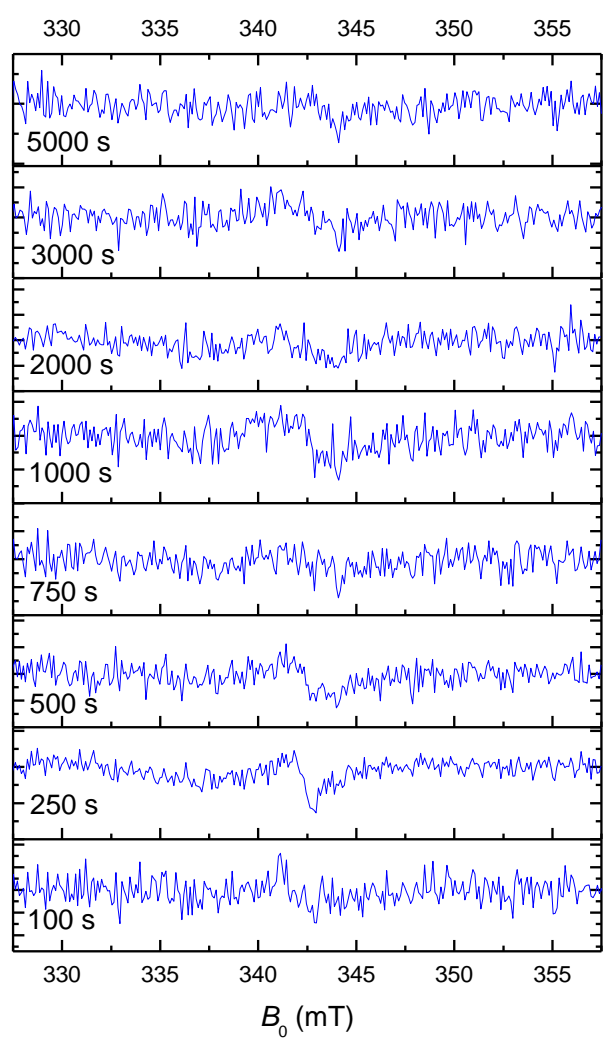




\subsection{Mass Spectrometry - Supplementary}

Table 5.1 Expected fragmentation pattern of EcLip3 after tryptic digestion

\begin{tabular}{cccc}
$\begin{array}{c}\text { Position of } \\
\text { cleavage site }\end{array}$ & Resulting peptide sequence & $\begin{array}{c}\text { Peptide length } \\
\text { [aa] }\end{array}$ & $\begin{array}{c}\text { Peptide mass } \\
\text { [Da] }\end{array}$ \\
\hline 7 & APAAGVK & 7 & 612.727 \\
27 & EVNVPDIGGDEVEVTEVMVK & 20 & 2158.406 \\
31 & VGDK & 4 & 417.462 \\
45 & VAAEQSLITVEGDK & 14 & 1459.616 \\
59 & ASMEVPAPFAGVVK & 14 & 1402.672 \\
62 & ELK & 3 & 388.464 \\
68 & VNVGDK & 6 & 630.699 \\
70 & VK & 2 & 245.322 \\
86 & TGSLIMIFEVEGAAPA & 16 & 1605.866 \\
\hline
\end{tabular}



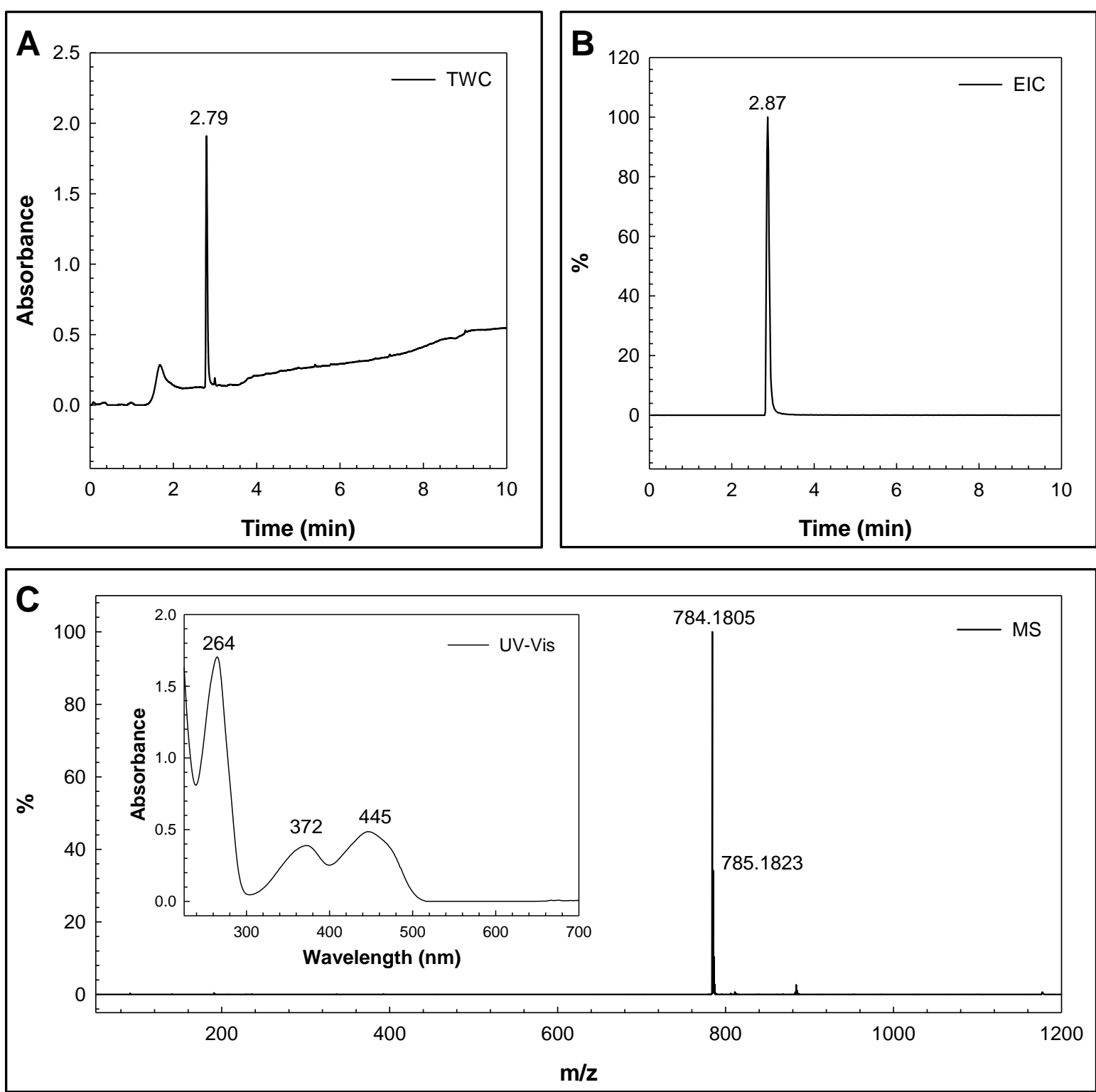

Figure 5.8 UPLC-ESI-TOF-MS analysis of FAD.

Flavin adenine dinucleotide disodium salt hydrate $\left(10 \mu \mathrm{g} / \mathrm{mL}\right.$ in $\left.\mathrm{ddH}_{2} \mathrm{O}\right)$ was purchased from AppliChem (Darmstadt). (A) Total wavelength chromatogram. (B) Extracted ion chromatogram for $\mathrm{m} / \mathrm{z}$ 784.150. (C) MS analysis (negative ionization mode) of B and corresponding UV-Vis spectrum of (inset). 

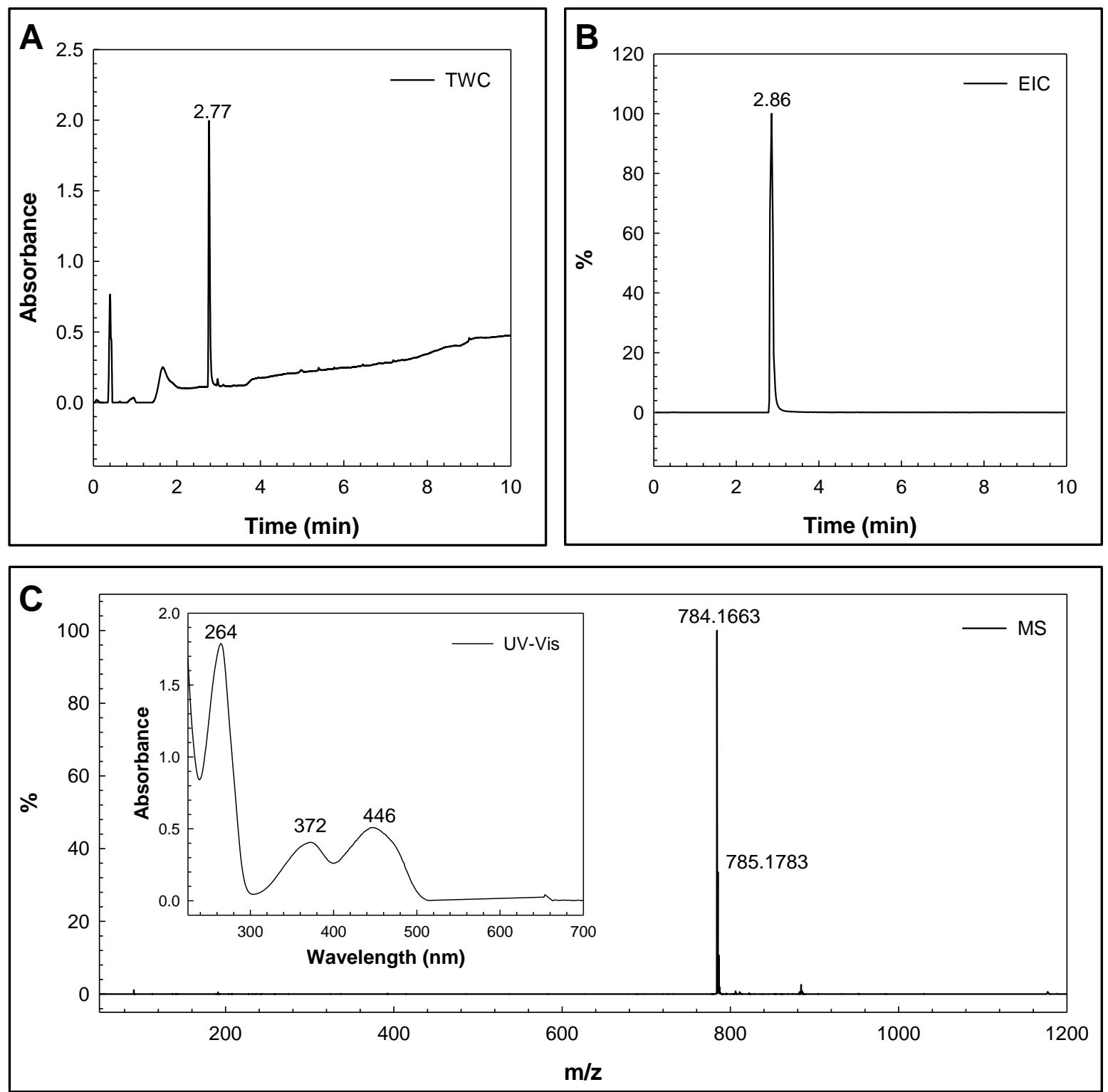

Figure 5.9 UPLC-ESI-TOF-MS analysis of FAD released of $E c \mathrm{E} 3 \mathrm{C} 45 \mathrm{~A} / \mathrm{K} 54 \mathrm{Q}$ by heat precipitation. (A) Total wavelength chromatogram. (B) Extracted ion chromatogram for $m / z 784.150$. (C) MS analysis (negative ionization mode) of B and corresponding UV-Vis spectrum of (inset). 

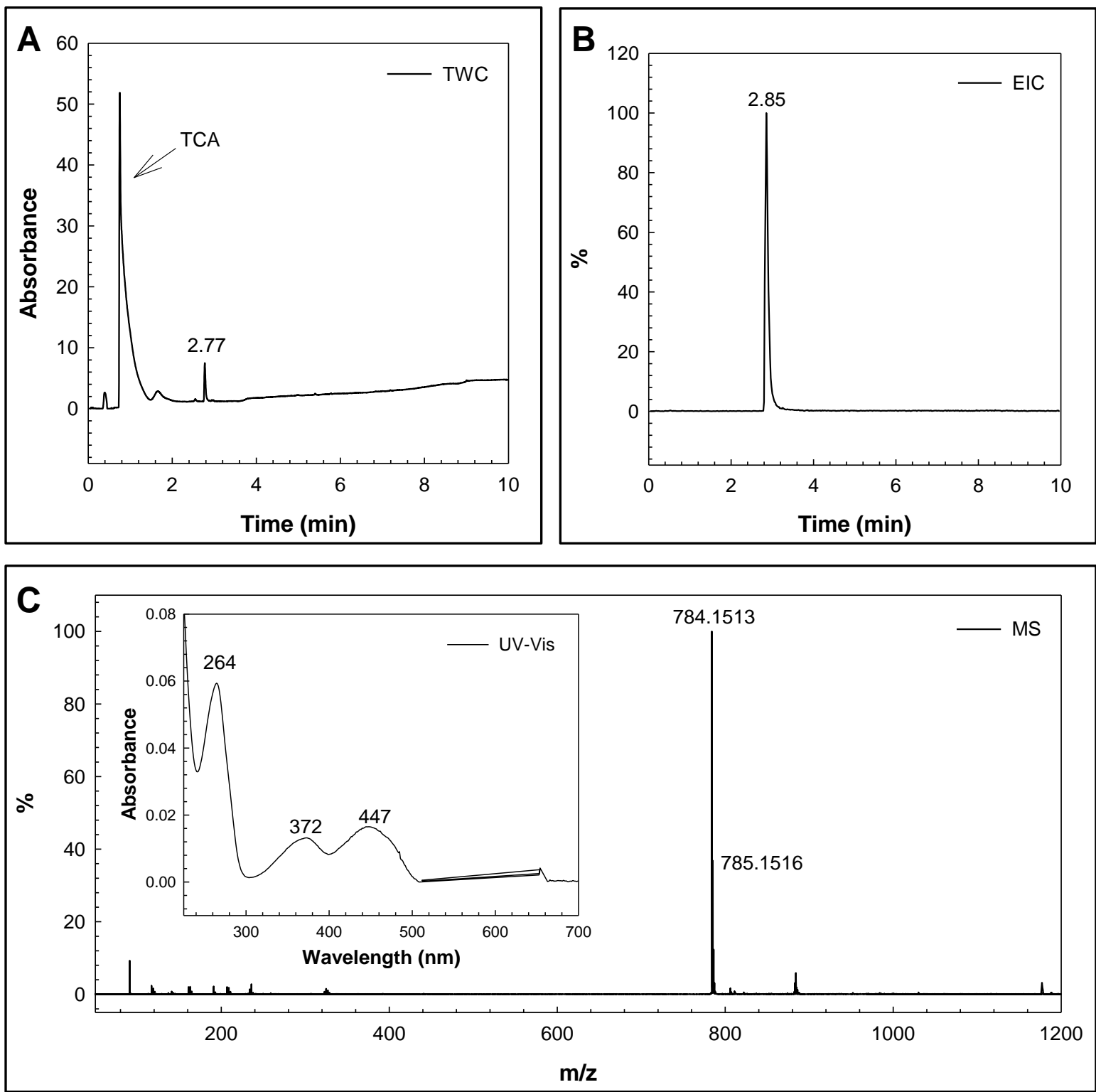

Figure 5.10 UPLC-ESI-TOF-MS analysis of FAD released of $E c$ E3C45A/K54Q by TCA precipitation.

(A) Total wavelength chromatogram. (B) Extracted ion chromatogram for $m / z$ 784.150. (C) MS analysis (negative ionization mode) of B and corresponding UV-Vis spectrum of (inset). 

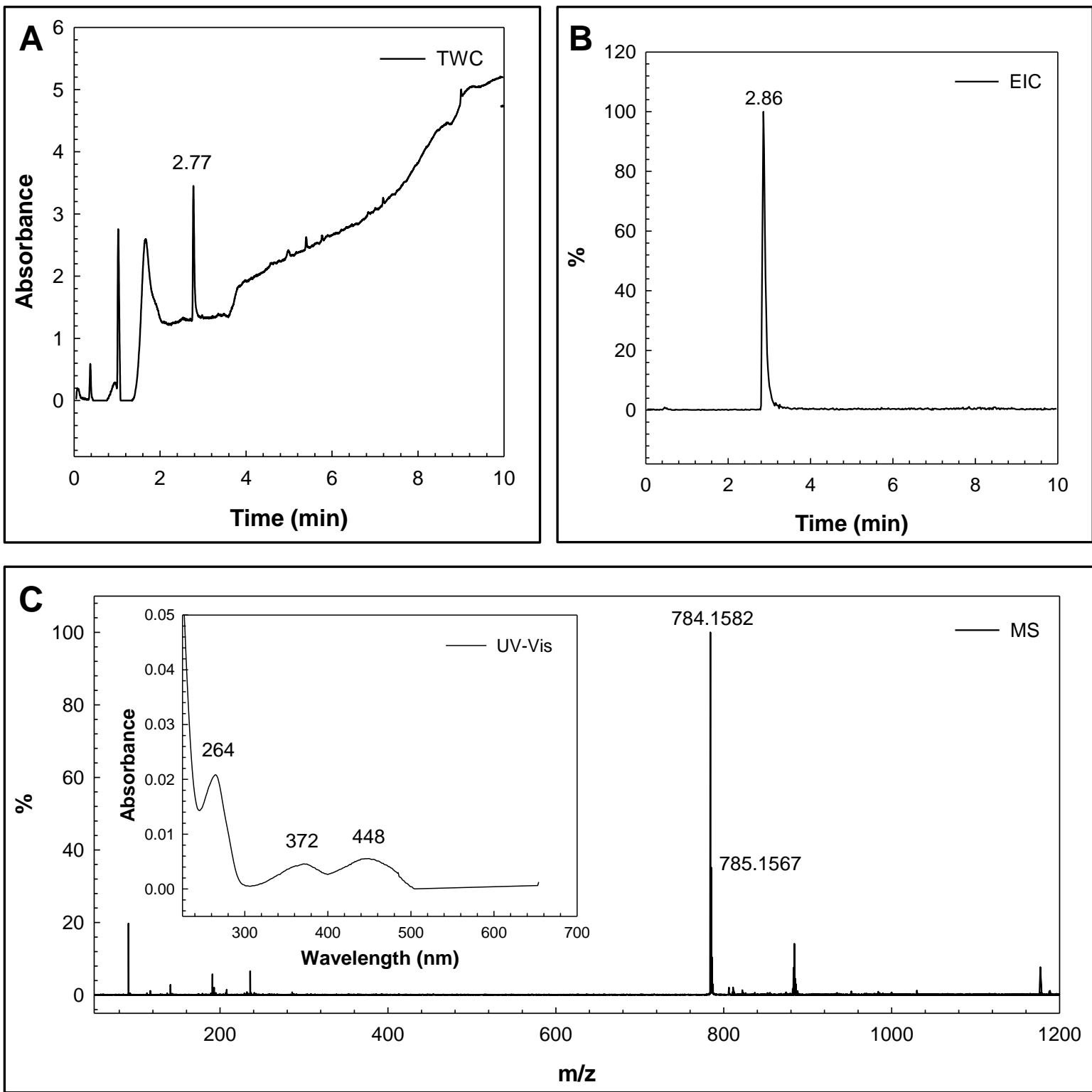

Figure 5.11 UPLC-ESI-TOF-MS analysis of FAD released of $E c E 3 C 45 \mathrm{~A} / \mathrm{K} 54 \mathrm{Q}$ by acetone precipitation.

(A) Total wavelength chromatogram. (B) Extracted ion chromatogram for $\mathrm{m} / \mathrm{z} 784.150$. (C) MS analysis (negative ionization mode) of B and corresponding UV-Vis spectrum of (inset). 

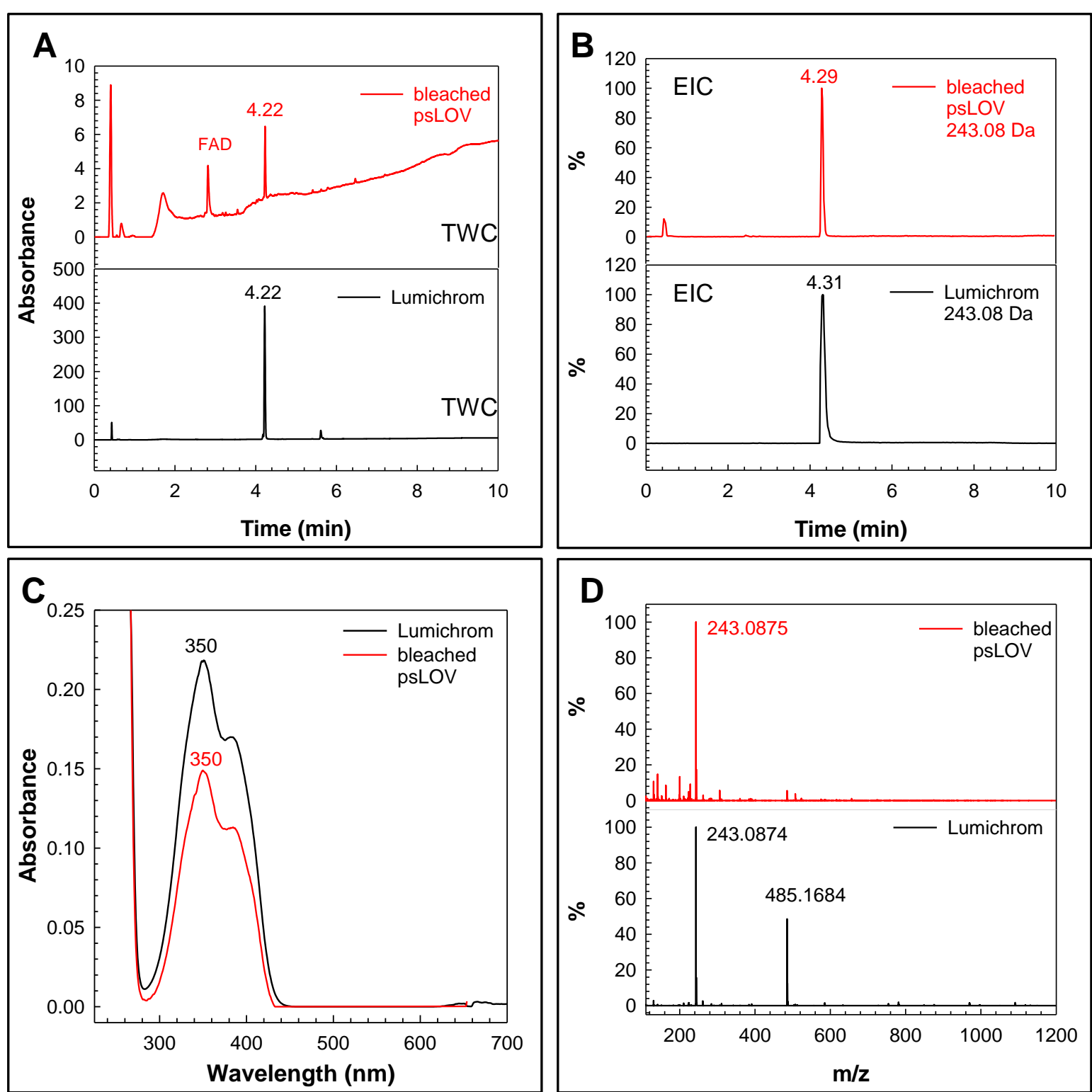

Figure 5.12 UPLC-ESI-TOF-MS comparison of lumichrom.

Released lumichrom from photobleached $E c \mathrm{E} 3 \mathrm{C} 45 \mathrm{~A} / \mathrm{K} 54 \mathrm{Q}$ by heat precipitation (red) and lumichrom $(100 \mu \mathrm{g} / \mathrm{mL}$ in $\mathrm{MeOH})$ purchased from Sigma (Deisenhofen) (black).

(A) Total wavelength chromatograms. (B) Extracted ion chromatograms for $m / z$ 243.087. (C) UVVis spectra extracted from (A). (D) MS analysis (positive ionization mode) of B. 

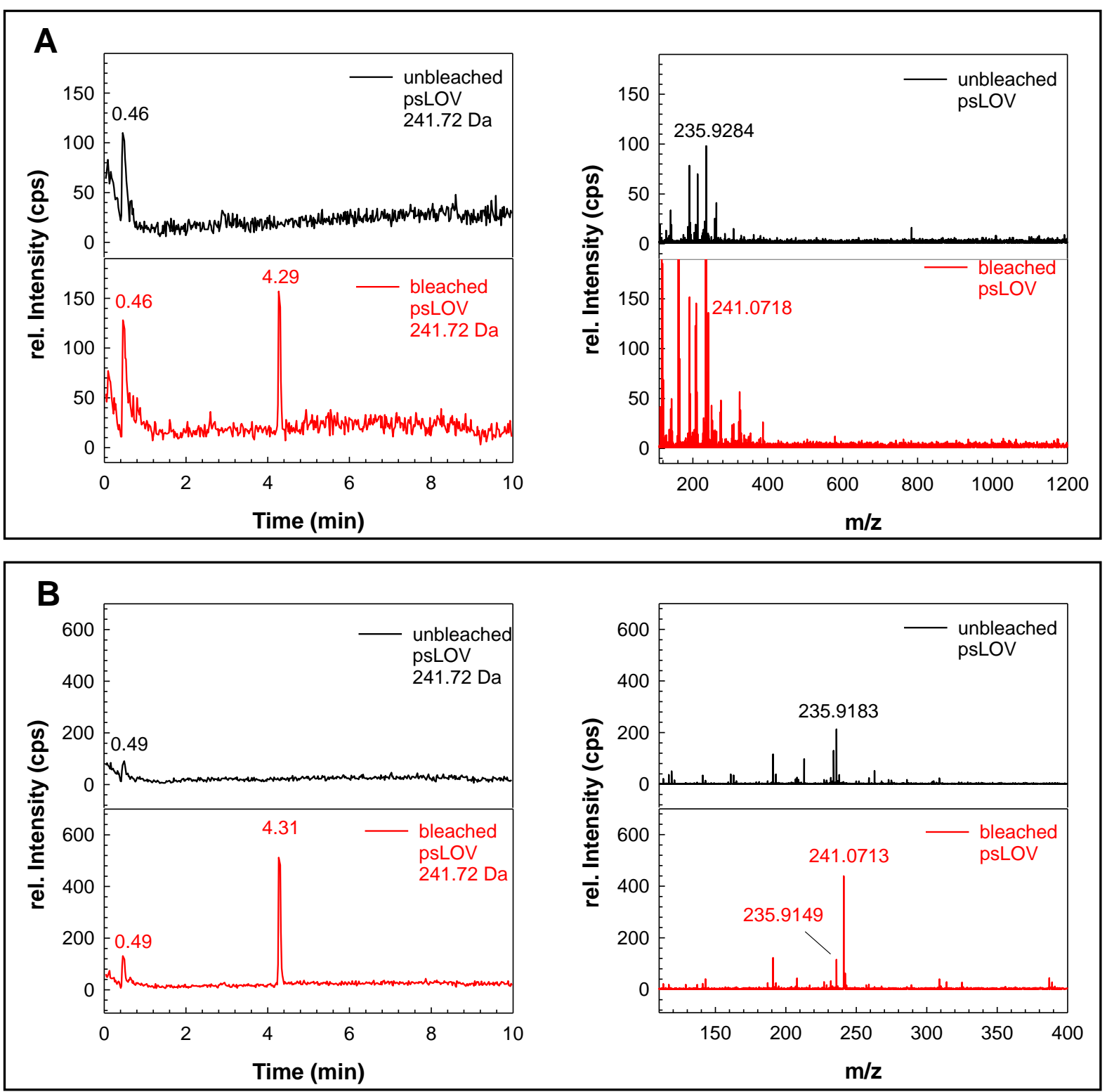

Figure 5.13 Evidence for lumichrom formation in EcE3C45A/K54Q by UPLC-ESI-TOF-MS.

Comparison of unbleached (black) and photobleached EcE3C45A/K54Q (red). (A) Extracted ion chromatograms (left) and corresponding MS analyses in negative ionization mode (right) of heat extracted samples $(\mathbf{A})$ and of TCA extracted samples $(\mathbf{B})$. 

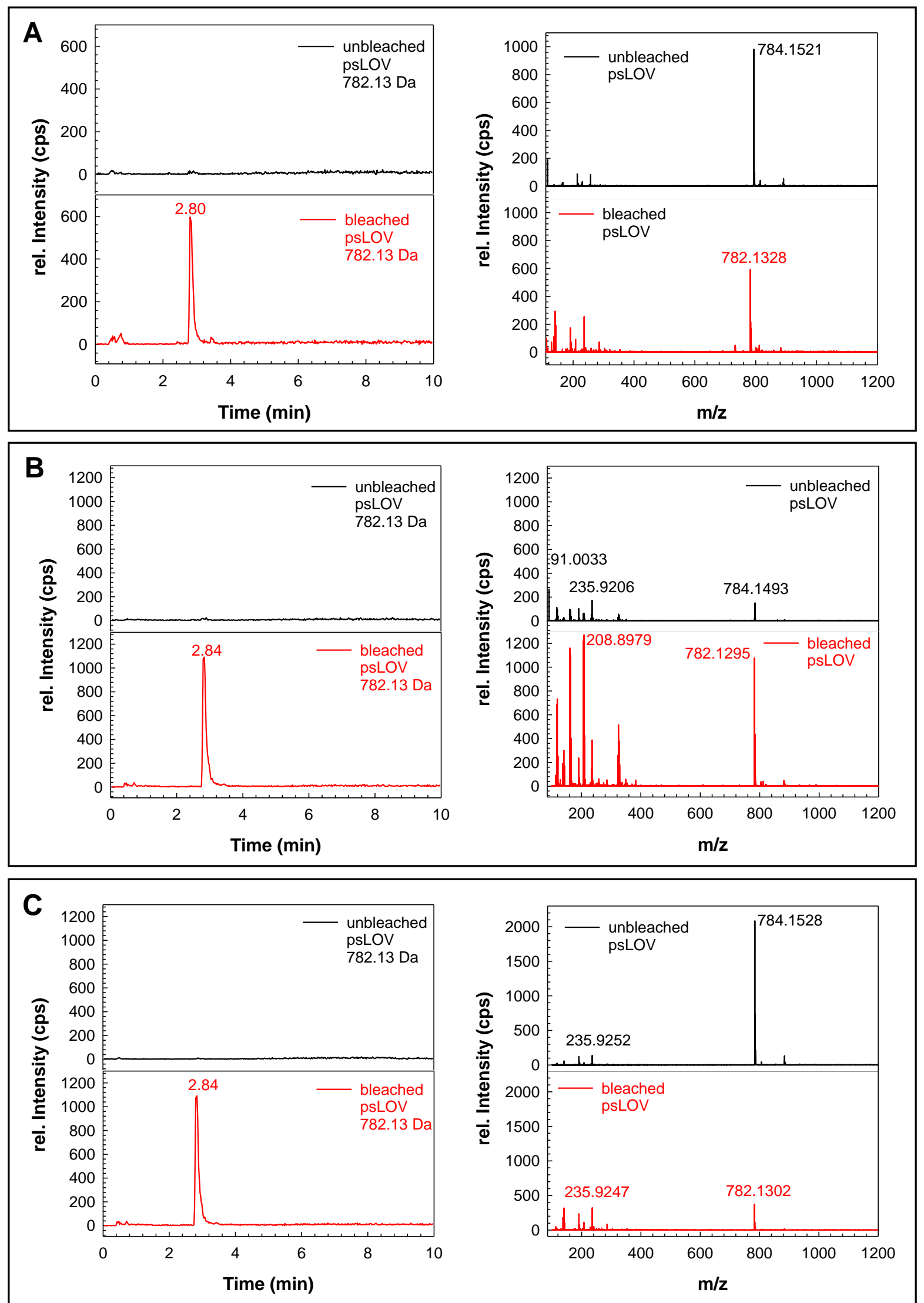

Figure 5.14 Evidence for FAD-2H formation in EcE3C45A/K54Q by UPLC-ESI-TOF-MS.

Comparison of unbleached (black) and photobleached $E c \mathrm{E} 3 \mathrm{C} 45 \mathrm{~A} / \mathrm{K} 54 \mathrm{Q}$ (red). Extracted ion chromatograms (left) and corresponding MS analyses in negative ionization mode (right) of heat extracted samples (A), of TCA extracted samples $(\mathbf{B})$ and of acetone extracted samples $(\mathbf{C})$. 

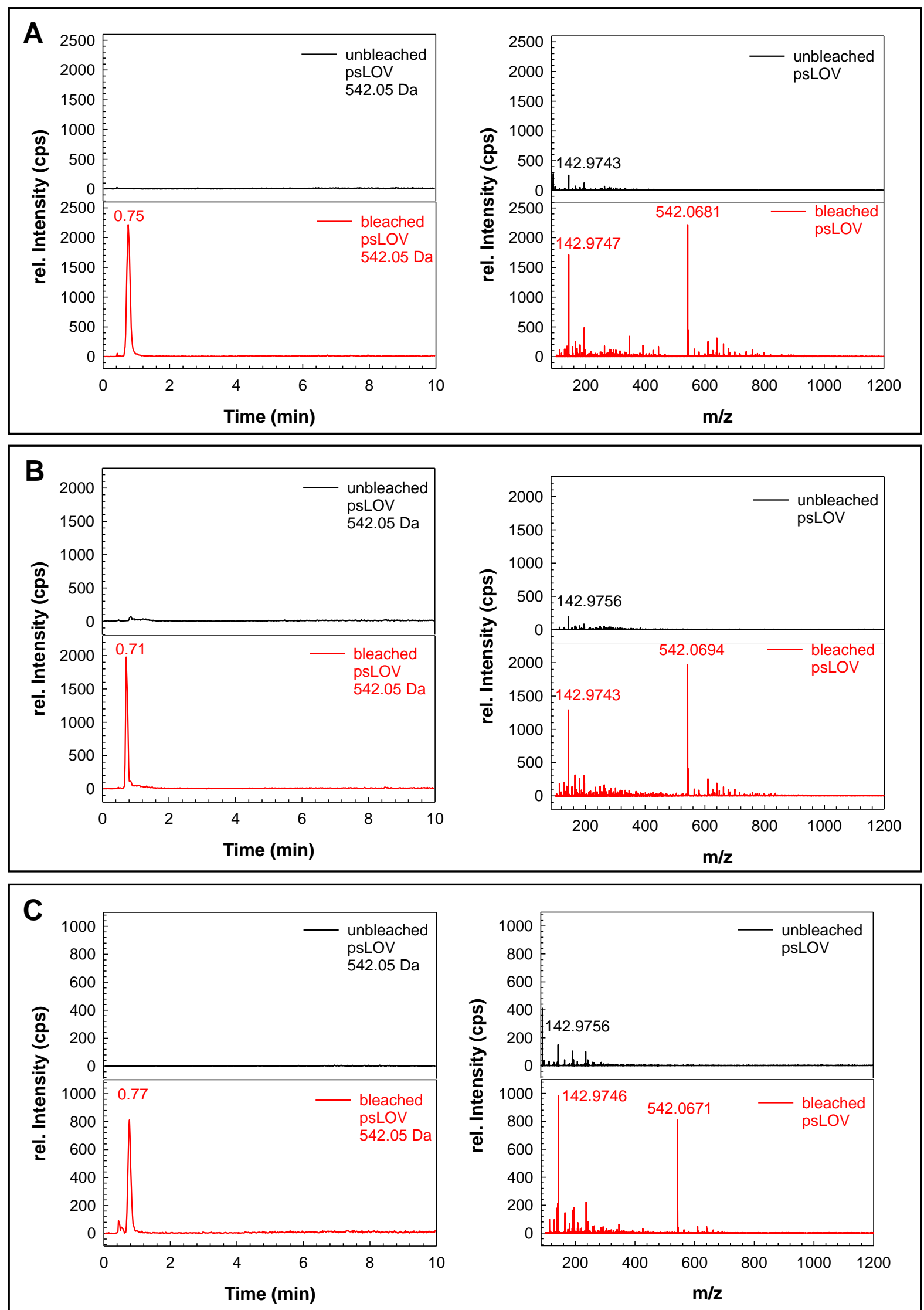

Figure 5.15 Ribityl-ADP-2H formation in EcE3C45A/K54Q by UPLC-ESI-TOF-MS.

Comparison of unbleached (black) and photobleached EcE3C45A/K54Q (red). Extracted ion chromatograms (left) and corresponding MS analyses in negative ionization mode (right) of heat extracted samples (A), of TCA extracted samples $(\mathbf{B})$ and of acetone extracted samples $(\mathbf{C})$. 


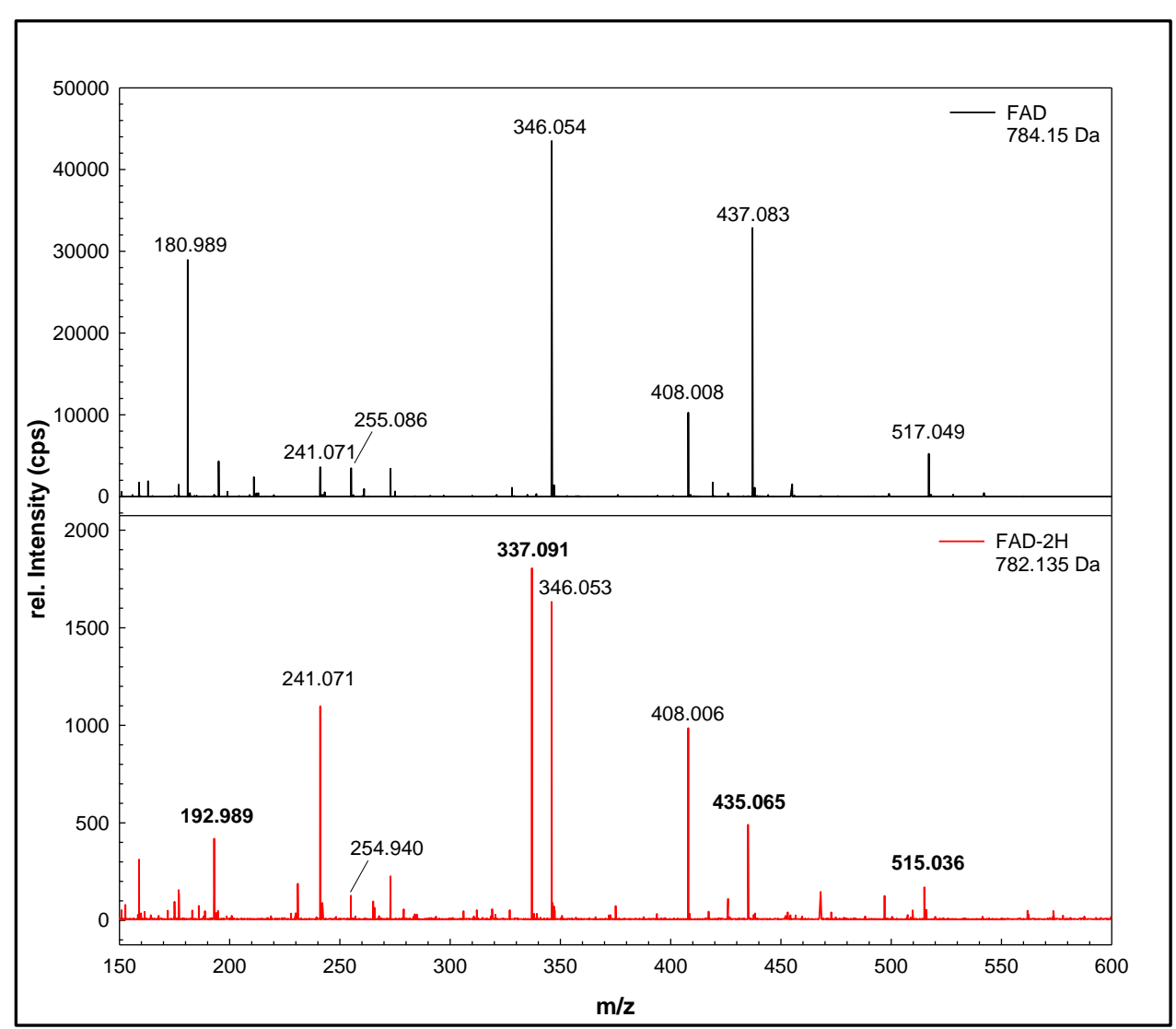

Figure 5.16 UHPLC-ESI-QTOF-MS/MS analysis of FAD and FAD-2H.

Extracted from photobleached $E c \mathrm{E} 3 \mathrm{C} 45 \mathrm{~A} / \mathrm{K} 54 \mathrm{Q}$.

MS/MS fragmentation pattern at $20 \mathrm{eV}$ collision energy at $\mathrm{m} / \mathrm{z} 784.150$ (black) and 782.135 (red), respectively. Deviating values are shown in bold face type. 


\subsection{Redox Potentials of selected EcE3 Variants}

Determination of the flavin redox potentials was performed as described in 2.2.2.9

Table 5.2 Redox potentials of relevant enzymes

\begin{tabular}{ccc} 
Enzyme & Redox potential $(\mathbf{m V})$ & Reference \\
\hline$E c$ E3wt & -314 & (Maeda-Yorita et al. 1994) \\
E3C45A/K54Q & -268 & This work \\
E3C50A & -345 & This work \\
E3C45S/C50S & -401 & This Work \\
LovK & -258 & (Purcell et al. 2010) \\
\hline
\end{tabular}
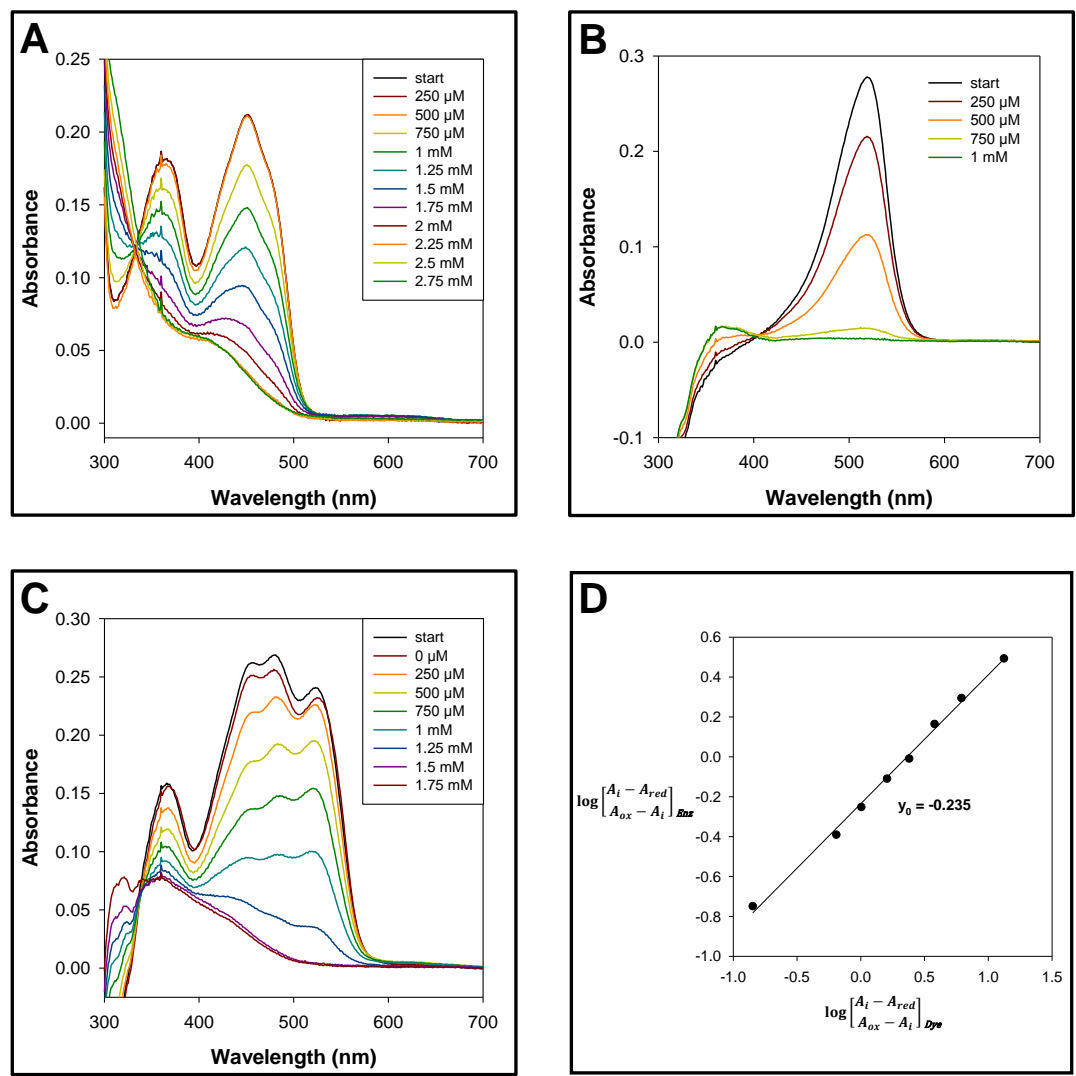

Figure 5.17 Determination of the redox potential of $E c \mathrm{E} 3 \mathrm{C} 45 \mathrm{~A} / \mathrm{K} 54 \mathrm{Q}$

Reduction of the enzyme (A) and of phenosafranin (B) under anaerobic conditions with dithionite. (C) Reduction of enzyme and dye under the same conditions. (D) Nernst analysis of the absorbance changes of the enzyme and dye according to 2.2.2.9. 

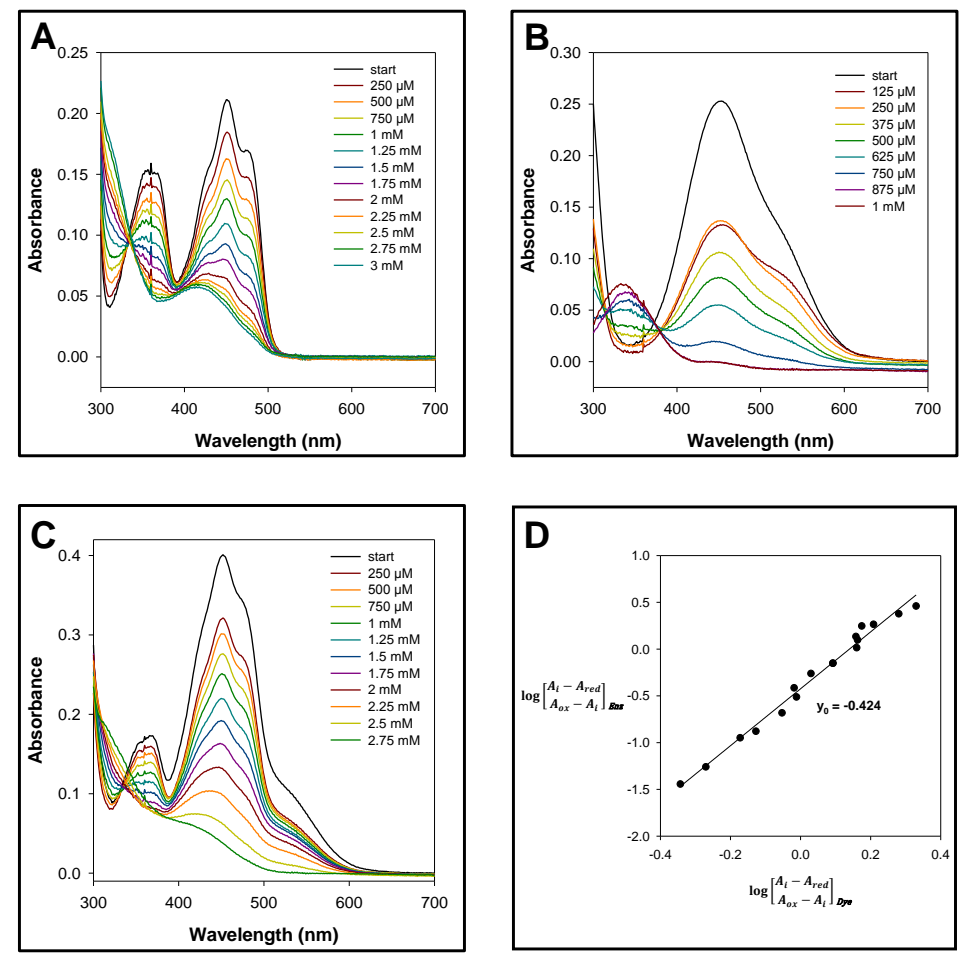

Figure 5.18 Determination of the redox potential of $E c$ E3C50A.

Reduction of the enzyme (A) and of neutral red (B) under anaerobic conditions with dithionite. (C) Reduction of enzyme and dye under the same conditions. (D) Nernst analysis of the absorbance changes of the enzyme and dye according to 2.2.2.9. 

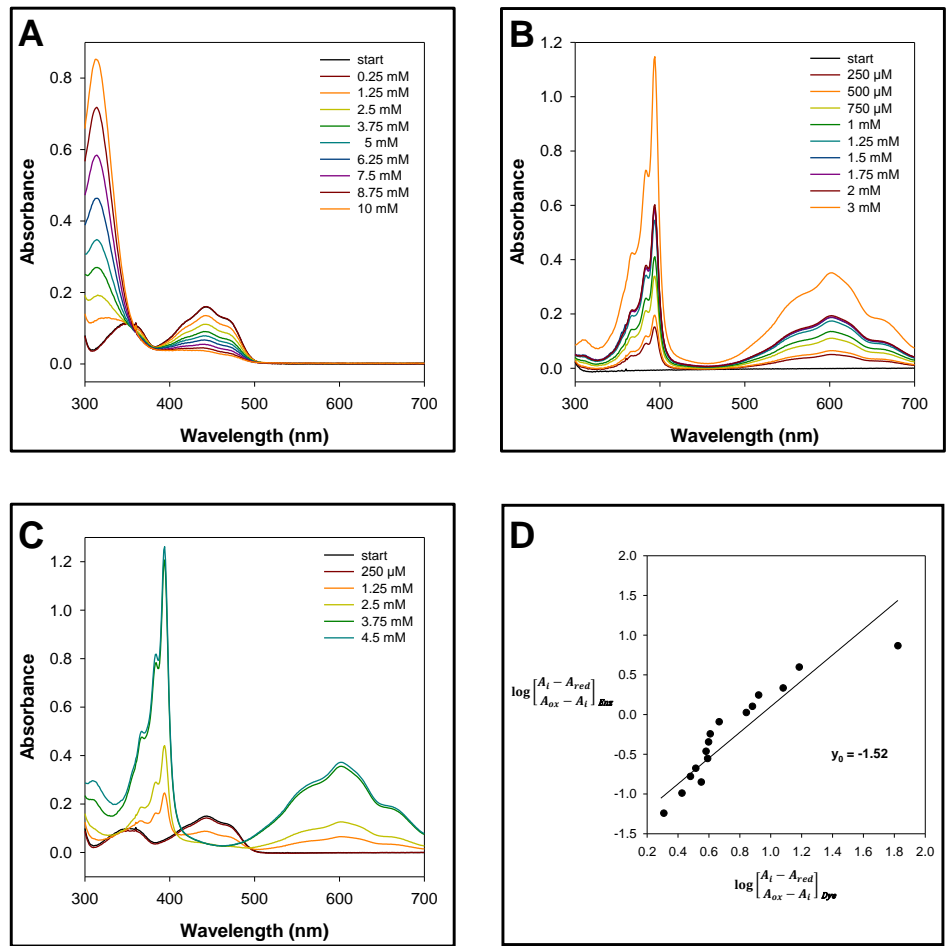

Figure 5.19 Determination of the redox potential of $E c \mathrm{E} 3 \mathrm{C} 45 \mathrm{~S} / \mathrm{C} 50 \mathrm{~S}$.

Reduction of the enzyme (A) and of methyl viologen (B) under anaerobic conditions with dithionite. (C) Reduction of enzyme and dye under the same conditions. (D) Nernst analysis of the absorbance changes of the enzyme and dye according to 2.2.2.9. 


\subsection{Isothermal Titration Calorimetry}

ITC experiments were performed according to 2.2.5.3.
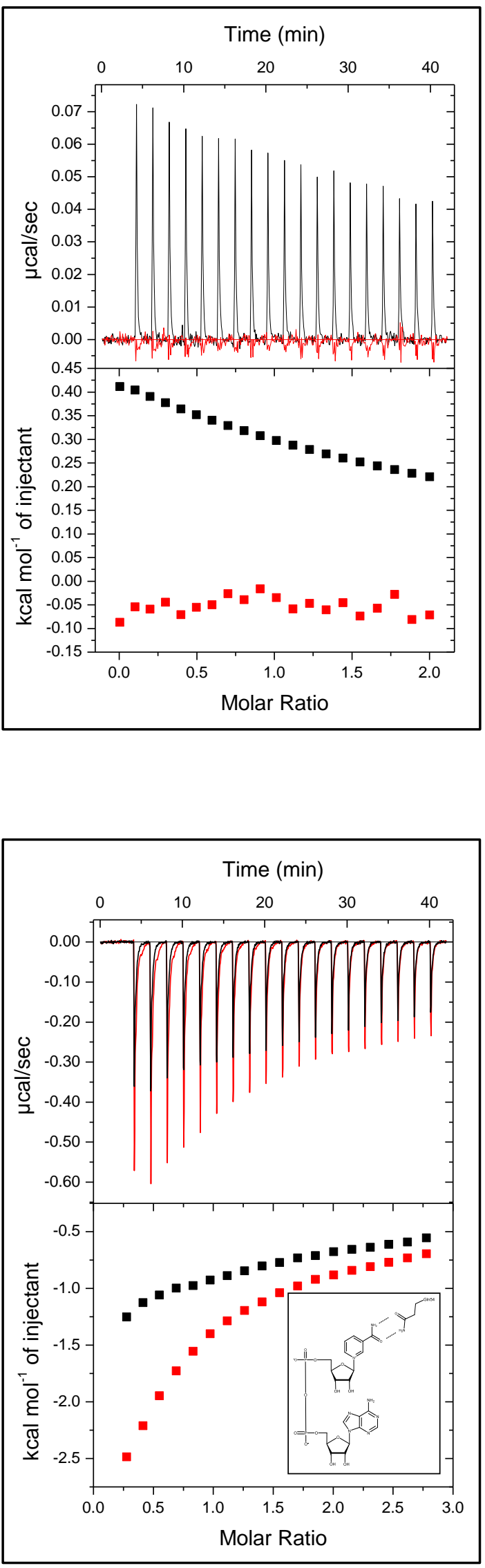

Figure 5.20 Titration of EcLip3 to $E c E 3$.

Both enzymes were desalted into the same buffer (50 mM KPP pH 7.6, $100 \mathrm{mM} \mathrm{NaCl}$ ). The ITC cell was filled with $100 \mu \mathrm{M}$ (active sites) EcE3. The injection syringe was filled with $1 \mathrm{mM}$ of EcLip3 Measurements were performed at $20{ }^{\circ} \mathrm{C}$ with a stirring speed of $500 \mathrm{~min}^{-1}$. A titration of $1 \mathrm{mM}$ EcLip3 into buffer served as control to check for heat effects due to dilution (red).

Figure 5.21 ITC titration of $E c E 3$ variants with $\mathrm{NAD}^{+}$

Titration of $\mathrm{NAD}^{+}$to $E c \mathrm{E} 3 \mathrm{wt}$ (black) and $E c \mathrm{E} 3 \mathrm{~K} 54 \mathrm{Q}$ (red).

$\mathrm{NAD}^{+}$was dissolved in $50 \mathrm{mM}$ KPP $\mathrm{pH} 7.6$, $100 \mathrm{mM} \mathrm{NaCl}$. The ITC cell was filled with $144 \mu \mathrm{M}$ (active sites) enzyme, respectively. The injection syringe was filled with $2 \mathrm{mM}$ of $\mathrm{NAD}^{+}$solution. Measurements were performed at $20^{\circ} \mathrm{C}$ with a stirring speed of $500 \mathrm{~min}^{-1}$.

The inset shows a putative interaction between Gln54 and the nicotine amide part of $\mathrm{NAD}^{+}$. 


\subsection{Crystallographic Tables}

Table 5.3 Crystallographic data and refinement statistics.

The highest resolution shell is shown in parenthesis.

$E c \mathrm{E} 3$ ground state $\quad E c \mathrm{E3}+\mathrm{NAD}^{+}$

$E c E 3 C 45 S$

Data collection

Wavelength $(\AA)$

Space Group

Cell dimensions

$\begin{array}{cc}\mathrm{a}(\AA) & 111.68 \\ \mathrm{~b}(\AA) & 129.32 \\ \mathrm{c}(\AA) & 258.34 \\ \mathrm{a}\left({ }^{\circ}\right) & 90.0 \\ \beta\left(^{\circ}\right) & 90.0 \\ \gamma\left({ }^{\circ}\right) & 90.0\end{array}$

Resolution range $(\AA)$

No. of reflections

50.00-2.17

(2.27-2.17)

743377

No. of unique reflections

Completeness (\%)

I/sigma (I)

$\mathrm{R}_{\text {merge }}(\%)$

$\mathrm{CC}_{1 / 2}$

Redundancy

B-factor from

Wilson Plot $\left(\AA^{2}\right)$

198344

$99.8(99.9)$

$19.3(2.7)$

$5.5(61.8)$

$99.9(73.3)$

$3.74(3.78)$

44.36

BESSYII PETRAIII

0.82480

$\mathrm{P} 2{ }_{1} 2_{1} 2_{1}$

I422

112.59

179.90

129.58

179.90

257.46

197.17

90.0

90.0

90.0

90.0

90.0

90.0

50.00-2.69

50.00-2.57

(2.81-2.69)

(2.67-2.57)

403884

1338625

103624

51245

98.5 (98.9)

99.7 (98.8)

$16.6(2.6)$

34.2 (6.7)

$6.4(69.2)$

$8.9(62.7)$

99.8 (70.3)

$100.0(96.8)$

3.89 (3.76)

$26.04(25.37)$

62.28

46.02 


\section{$E c$ E3 ground state $\quad E c E 3+\mathrm{NAD}^{+} \quad E c \mathrm{E} 3 \mathrm{C} 45 \mathrm{~S}$}

\section{Refinement}

Resolution Range

(A)

Monomers per AU

Reflections

$$
\mathrm{R}_{\text {work }} / \mathrm{R}_{\text {free }}(\%)
$$

Number of atoms

$$
\text { protein }
$$

ligands

water

B-factor protein

B-factor ligands

B-factor water

Deviations from

$$
\text { ideals (r.m.s.d) }
$$

Bond distances ( $\mathrm{A}$ )

Bond angles $\left({ }^{\circ}\right)$

Dihedrals $\left({ }^{\circ}\right)$
$42.51-2.17$

6

198214

$17.7 / 20.8$

21417

569

1752

38.89

41.65

40.42

0.006

1.083

13.902

96.73

98.12

88.47

3.24

0.04

21303

466

24

66.29

66.42

54.13

0.010

0.012

1.271

1.331

15.926

17.072
1.88

8.51

0.00

3.02
Ramachandran plot

favoured regions (\%)

allowed regions (\%)

outlier regions (\%) 


\subsection{X-ray Figures}

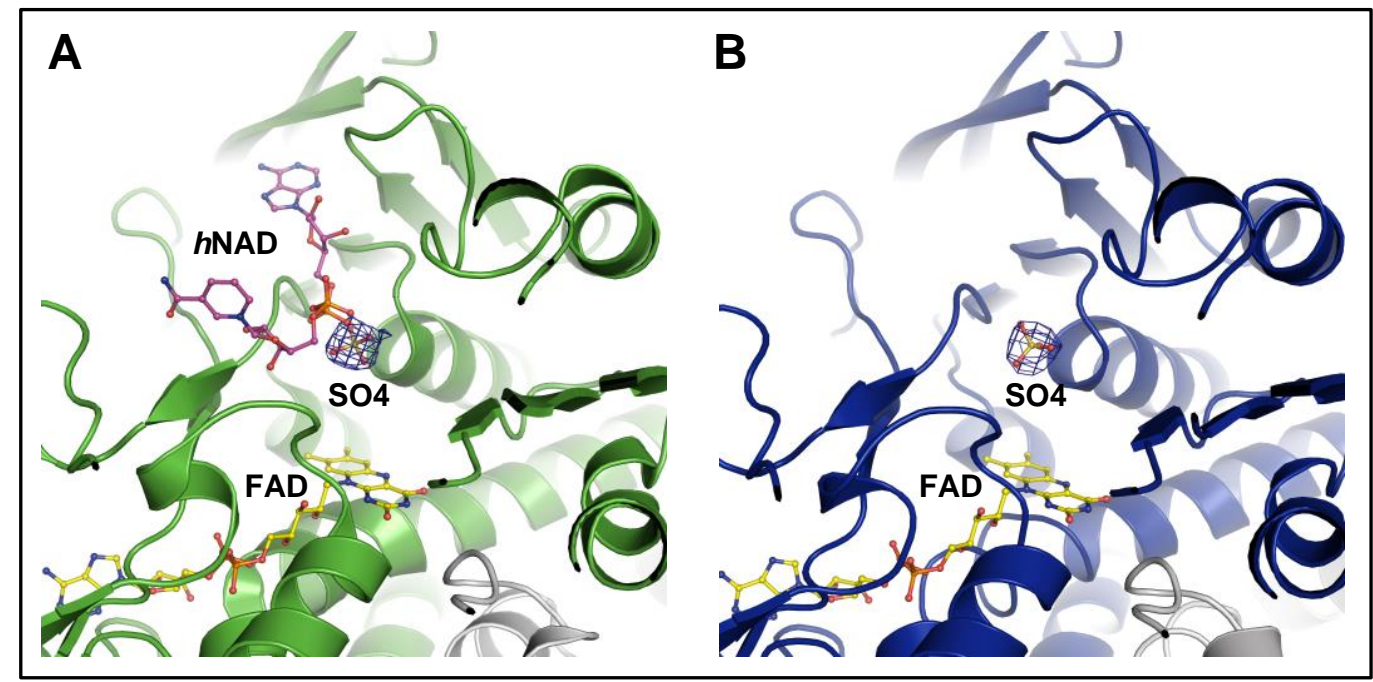

Figure 5.22 The placeholder ability of sulfate ions serving as precipitant.

Top views on the re face of the flavin showing the nucleotide binding site. (A) Cartoon representation of $E c \mathrm{E} 3$ in ground state showing the presence of a sulfate ion at the NAD-binding site. A superposition with the human E3 structure in complex with NAD ${ }^{+}$ (PDB: 1ZMC) confirms the placeholder abilities of the precipitant. For convenience only the nucleotide is presented. (B) Repeating motive of the precipitant in the adduct structure. $2 \mathrm{mFo}-\mathrm{DFc}$ maps are countered at $2 \sigma$ (blue mesh), respectively.

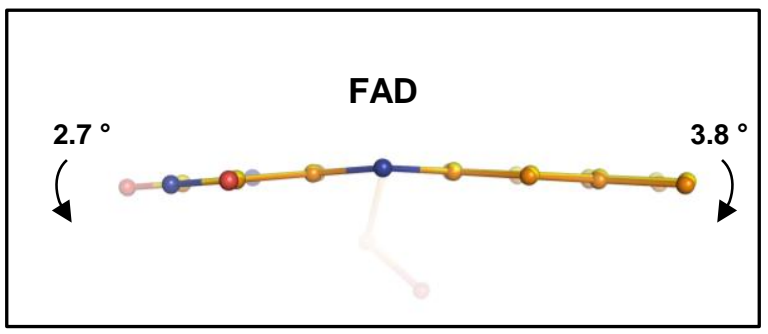

Figure 5.23 Further bending of the flavin cofactor at the two-electron reduced state.

Ball and stick representation of the FADs from $\mathrm{EcE} 3$ at ground state (yellow) and in EcE3C45S (orange). The dimethylbenzene part is $\sim 1.3^{\circ}$ further bent compared to the wild type FAD indicating its altered electronic state. 

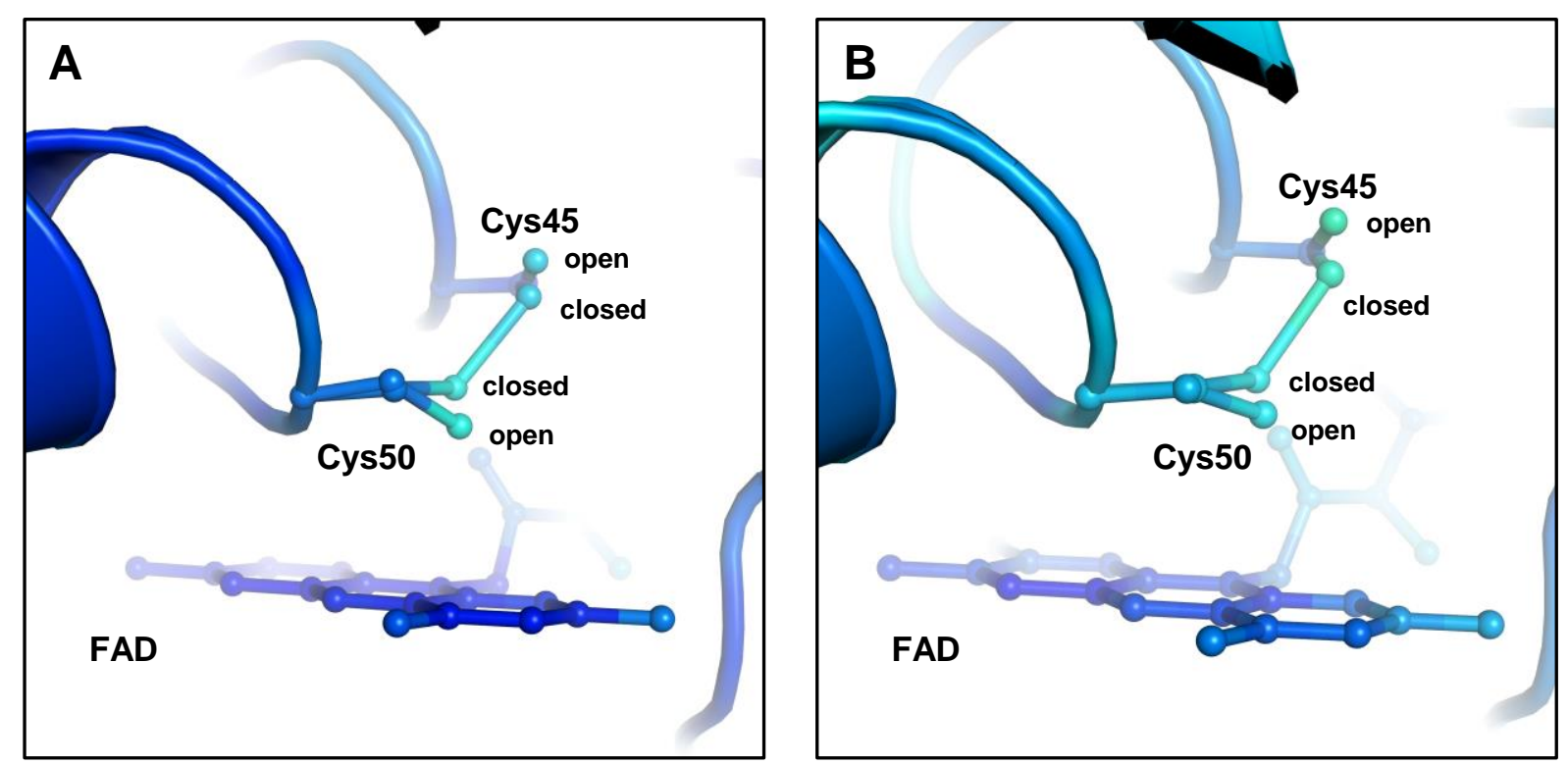

Figure 5.24 B-factor representation of the $E c E 3$ active site at $2.69 \AA$.

Atoms of the redox active cystine and the flavin cofactor are color-coded according to their individual B-factors. Both cysteine residues in molecule A (A) and in molecule F (B) of the asymmetric unit do not show elevated atomic displacement parameters relative to their enzyme surrounding. 

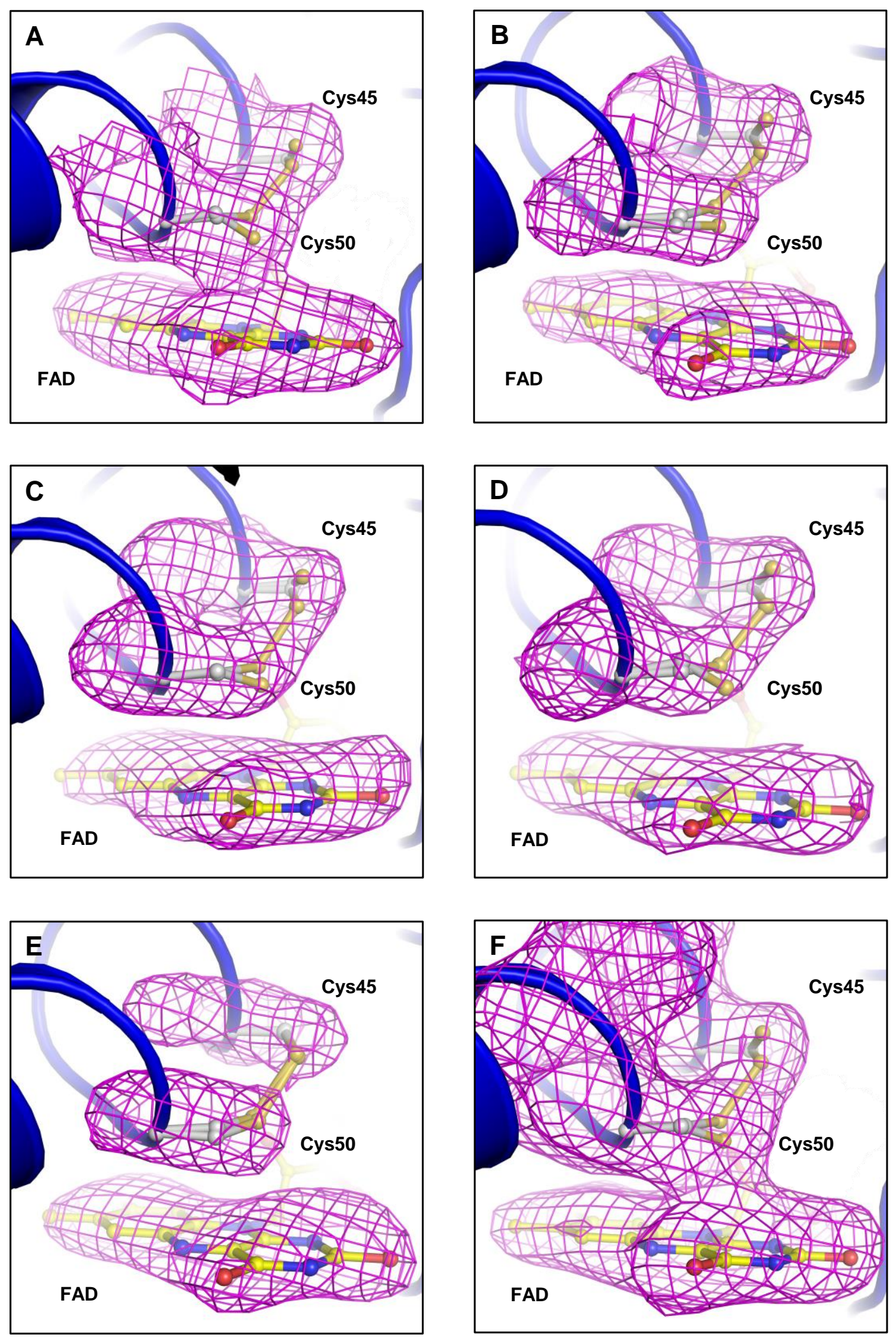

Figure 5.25 Active site of EcE3 at $2.69 \AA$.

Simulated annealing OMIT maps for all molecules in the asymmetric unit (mFo-DFc at $3 \sigma$ ). Figure numbering reflects the respective molecule label. Cohesive density is present in chain A and chain F, suggesting the formation of a covalent adduct. 


\section{Bibliography}

Adams, P. D. et al. 2010. "PHENIX: A Comprehensive Python-Based System for Macromolecular Structure Solution.” Acta Crystallographica Section D: Biological Crystallography 66:213-21.

Ahmad, I., S. Ahmed, M. A. Sheraz, F. H. M. Vaid, and I. A. Ansari. 2010. "Effect of Divalent Anions on Photodegradation Kinetics and Pathways of Riboflavin in Aqueous Solution." International Journal of Pharmaceutics 390(2):174-82.

Ahmad, I., Q. Fasihullah, A. Noor, A. Ansari, and Q. Nawab Manzar Ali. 2004. "Photolysis of Riboflavin in Aqueous Solution: A Kinetic Study." International Journal of Pharmaceutics 280(1-2):199-208.

Ahmad, R. and D. A. Armstrong. 1984. "Reduction of Lumichrome by the Radical Anions of CO2 and Lipoamide." International journal of radiation biology and related studies in physics, chemistry, and medicine 45:607-14.

Akiyama, S. K. and G. G. Hammes. 1981. "Elementary Steps in the Reaction Mechanism of Pyruvate Dehydrogenase Multienzyme Complex from Escherichia coli: Kinetics of Flavin Reduction.” Biochemistry 20(May):1491-97.

Alexandre, M.T.A., J. C. Arents, R. Van Grondelle, K.s J. Hellingwerf, and J. T. M. Kennis. 2007. “A Base-Catalyzed Mechanism for Dark State Recovery in the Avena Sativa Phototropin-1 LOV2 Domain.” Biochemistry 46:3129-37.

Alexandre, M.T.A. et al. 2009. "Primary Reactions of the LOV2 Domain of Phototropin Studied with Ultrafast Mid-Infrared Spectroscopy and Quantum Chemistry.” Biophysical Journal 97(1):22737.

Ali, S. T. and J. R. Guest. 1990. "Isolation and Characterization of Lipoylated and Unlipoylated Domains of the E2p Subunit of the Pyruvate Dehydrogenase Complex of Escherichia coli." The Biochemical Journal 271:139-45.

Aliverti, A., 2010. "Identifying and Quantitating FAD and FMN in Simple and in Iron-SulfurContaining Flavoproteins.” Pp. 9-23 in Flavoprotein Protocols. Springer Verlag GmbH.

Argyrou, A. and J. S. Blanchard. 2001. "Mycobacterium Tuberculosis Lipoamide Dehydrogenase Is Encoded by Rv0462 and Not by the lpdA or lpdB Genes." Biochemistry 40:11353-63. 
Argyrou, A., J S. Blanchard, and B. A. Palfey. 2002. "The Lipoamide Dehydrogenase from Mycobacterium Tuberculosis Permits the Direct Observation of Flavin Intermediates in Catalysis." Biochemistry 41:14580-90.

Argyrou, A., G. Sun, B. A. Palfey, and J. S. Blanchard. 2003. "Catalysis of Diaphorase Reactions by Mycobacterium Tuberculosis Lipoamide Dehydrogenase Occurs at the EH4 Level." Biochemistry 42:2218-28.

Bates, D. L., M. J. Danson, G. Hale, E. A. Hooper, and R. N. Perham. 1977. "Self-Assembly and Catalytic Activity of the Pyruvate Dehydrogenase Multienzyme Complex from Eschericia Coli." Nature 268:313-16.

Bauer, C., C. R. Rabl, J. Heberle, and T. Kottke. 2011. "Indication for a Radical Intermediate Preceding the Signaling State in the LOV Domain Photocycle." Photochemistry and Photobiology 87(26):548-53.

Beel, B. et al. 2012. "A Flavin Binding Cryptochrome Photoreceptor Responds to Both Blue and Red Light in Chlamydomonas Reinhardtii." The Plant Cell 24(July):2992-3008.

Benen, J. et al. 1991. "Lipoamide Dehydrogenase from Azotobacter-Vinelandii - Site-Directed Mutagenesis of the His450-Glu455 Diad - Spectral Properties of Wild Type and Mutated Enzymes." European Journal of Biochemistry 202:863-72.

Benen, J., W. Van Berkel, N. Dieteren, et al. 1992. "Lipoamide Dehydrogenase from Azotobacter Vinelandii: Site-Directed Mutagenesis of the His450-Glu455 Diad. Kinetics of Wild-Type and Mutated Enzymes.” European Journal of Biochemistry 207:487-97.

Benen, J., W. Van Berkel, C. Veeger, and A. de Kok. 1992. "Lipoamide Dehydrogenase from Azotobacter Vinelandii The Role of the C-Terminus in Catalysis and Dimer Stabilization." European Journal of Biochemistry 207:499-505.

Bird, C. L. and A. T. Kuhn. 1981. "Electrochemistry of the Viologens." Chemical Society Reviews $10: 49$.

Bisswanger, H. 1981. "Substrate Specificity of the Pyruvate Dehydrogenase Complex from Escherichia Coli." The Journal of Biological Chemistry 256(2):815-22.

Brautigam, C. A., J. L. Chuang, D. R. Tomchick, M. Machius, and D. T. Chuang. 2005. "Crystal Structure of Human Dihydrolipoamide Dehydrogenase: NAD +/NADH Binding and the Structural Basis of Disease-Causing Mutations.” Journal of Molecular Biology 350:543-52. 
Brünger, A.T. 1992. "Free R Value: Anovel Statistical Quantity for Assessing the Accurancy of Crystal Structures." Nature 355:472-75.

Brunger, A.T. 2007. "Version 1.2 of the Crystallography and NMR System." Nature protocols 2:2728-33.

Cairns, W. L. and D. E. Metzler. 1971. "Photochemical Degradation of Flavins. VI. A New Photoproduct and Its Use in Studying the Photolytic Mechanism." Journal of the American Chemical Society 93(5):2772-77.

Chandrasekhar, K. et al. 2013. "Insight to the Interaction of the Dihydrolipoamide Acetyltransferase (E2) Core with the Peripheral Components in the Escherichia Coli Pyruvate Dehydrogenase Complex via Multifaceted Structural Approaches.” Journal of Biological Chemistry 288:1540217.

Choong, Y. S. and V. Massey. 1980. "Stabilization of Lactate Oxidase Flavin Anion Radical by Complex Formation.” Journal of Biological Chemistry 255:8672-77.

Clark, DP. 1989. "The Fermentation Pathways of Escherichia Coli." FEMS Microbiology Reviews 63:223-34.

Conrad, K. S., C. C. Manahan, and B. R. Crane. 2014. "Photochemistry of Flavoprotein Light Sensors." Nature Chemical Biology 10(10):801-9.

Cooper, R. H., P. J. Randle, and R. M. Denton. 1974. "Regulation of Heart Muscle Pyruvate Dehydrogenase Kinase.” The Biochemical Journal 143:625-41.

Corpet, F. 2010. “Nucleic Acids Research.” Nucleic Acids Research 38(22).

Crosson, S. and K. Moffat. 2001. "Structure of a Flavin-Binding Plant Photoreceptor Domain: Insights into Light-Mediated Signal Transduction." Proceedings of the National Academy of Sciences of the United States of America 98(6):2995-3000.

Crosson, S. and K. Moffat. 2002. "Photoexcited Structure of a Plant Photoreceptor Domain Reveals a Light-Driven Molecular Switch.” The Plant Cell 14(May):1067-75.

Davis, I. W. et al. 2007. "MolProbity: All-Atom Contacts and Structure Validation for Proteins and Nucleic Acids." Nucleic Acids Research 35(13):375-83.

DeLano, W. L. 2003. “The PyMOL Molecular Graphics System.” http://www.pymol.org/. 
Diensthuber, R. P., M. Bommer, T. Gleichmann, and A. Möglich. 2013. "Full-Length Structure of a Sensor Histidine Kinase Pinpoints Coaxial Coiled Coils as Signal Transducers and Modulators." Structure 21:1127-36.

Ehrenberg, A., F. Müller, and P. Hemmerich. 1967. "Basicity, Visible Spectra, and Electron Spin Resonance of Flavosemiquinone Anions.” European journal of biochemistry / FEBS 2:286-93.

Ellis, K. J. and J. F. Morrison. 1982. "Buffers of Constant Ionic Strength for Studying pH-Dependent Processes." 87:405-26 in Methods in Enzymology.

Emsley, P., B. Lohkamp, W. G. Scott, and K. Cowtan. 2010. "Features and Development of Coot." Acta Crystallographica Section D: Biological Crystallography 66:486-501.

Fraenkel, DG. 1996. "Escherichia Coli and Salmonella: Cellular and Molecular Biology.” Pp. 189-98 in eds Neidhardt FC, et al. Washington, DC: ASM Press.

Ghisla, S., B. Entsch, V. Massey, and M. Husein. 1977. "On the Structure of Flavin-Oxygen Intermediates Involved in Enzymatic Reactions." European Journal of Biochemistry / FEBS 76:139-48.

Ghisla, S. and V. Massey. 1975. "Mechanism of Inactivation of the Flavoenzyme Lactate Oxidase by Oxalate." Journal of Biological Chemistry 250:577-84.

Ghisla, S., V. Massey, J. M. Lhoste, and S. G. Mayhew. 1974. "Fluorescence and Optical Characteristics of Reduced Flavines and Flavoproteins." Biochemistry 13(3):589-97.

Gibson, Q. H., V. Massey, and N. M. Atherton. 1962. "The Nature of Compounds Present in Mixtures of Oxidized and Reduced Flavin Mononucleotides." The Biochemical Journal 85(1958):369-83.

Graef, M. R. De, S. Alexeeva, J. L. Snoep, and M. J. Teixeira De Mattos. 1999. "The Steady-State Internal Redox State ( NADH/NAD ) Reflects the External Redox State and Is Correlated with Catabolic Adaptation in Escherichia Coli.” Journal of Bacteriology 181(8):2351-57.

Green, J. D., E. D. Laue, R. N. Perham, S. T. Ali, and J. R. Guest. 1995. "Three-Dimensional Structure of a Lipoyl Domain from the Dihydrolipoyl Acetyltransferase Component of the Pyruvate Dehydrogenase Multienzyme Complex of Escherichia Coli." Journal of Molecular Biology 248:328-43.

Güttler, B. H.-O. 2008. "Untersuchungen Zum Mechanismus Der Reduktiven Acetylierung Der Acyltransferase Des Humanen Pyruvat-Dehydrogenase-Komplexes.” Martin-Luther-Universität Halle-Wittenberg. 
Hald, E., P. Lehmann, and J. Ziegenhorn. 1975. "Molar Absorptivities of $\beta$-NADH and / $\beta$-NAD at 260 Nm." Clinical Chemistry 7:884-87.

Hansen, HG and U. Henning. 1966. "Regulation of Pyruvate Dehydrogenase Activity in Escherichia Coli K12.” Biochim Biophys Acta 122:355-58.

Hanukoglu, I. 2015. "Proteopedia: Rossmann Fold: A Beta-Alpha-Beta Fold at Dinucleotide Binding Sites.” Biochem Mol Biol Educ. 43:206-9.

Harbury, H., K. LaNoue, P. Loach, and R. Amick. 1959. "Molecular Interaction Of Isioalloxazine Derivatives." Proceedings of the National Academy of Sciences 45:1708-17.

Hasford, Justin J., W. Kemnitzer, and C. J. Rizzo. 1997. "Conformational Effects on Flavin Redox Chemistry." The Journal of Organic Chemistry 62(8):5244-45.

Hitomi, K., S. Kim, N. Harima, M. Ikenaga, and T. Todo. 1997. "Binding and Catalytic Properties of Xenopus (6-4) Photolyase.” 272(51):32591-98.

Hopkins, N. and C. H. Williams. 1995a. "Characterization of Lipoamide Dehydrogenase from Escherichia Coli Lacking the Redox Active Disulfide: C44S and C49S." Biochemistry 34(151):11757-65.

Hopkins, N. and C. H. Williams. 1995b. "Lipoamide Dehydrogenase from Escherichia Coli Lacking the Redox Active Disulfide: C44S and C49S. Redox Properties of the FAD and Interactions with Pyridine Nucleotides." Biochemistry 34(151):11766-76.

Hucho, F., D.D. Randall, T.E. Roche, M.W. Burgett, J.W. Pelley, L.J. Reed. 1972. “ $\alpha$-Keto acid dehydrogenase complexes. XVII. Kinetic and regulatory properties of pyruvate dehydrogenase kinase and pyruvate dehydrogenase phosphatase from bovine kidney and heart." Archives of Biochemistry and Biophysics 151:328-340.

Huang, R., J. K. Hyun, and D. B. Min. 2006. "Photosensitizing Effect of Riboflavin, Lumiflavin, and Lumichrome on the Generation of Volatiles in Soy Milk." Journal of Agricultural and Food Chemistry 54:2359-64.

Inoue, H., H. Nojima, and H. Okayama. 1990. "High Efficiency Transformation of Escherichia coli with Plasmids." Gene 96:23-28.

Jung, M. Y., Y. S. Oh, D. K. Kim, H.-J. Kim, and D. B. Min. 2007. "Photoinduced Generation of 2,3Butanedione from Riboflavin.” Journal of Agricultural and Food Chemistry 55:170-74.

Kabsch, W. 2010. “Xds.” Acta Crystallographica Section D: Biological Crystallography 66:125-32. 
Kato, Masato et al. 2008. "Structural Basis for Inactivation of the Human Pyruvate Dehydrogenase Complex by Phosphorylation: Role of Disordered Phosphorylation Loops." Structure 16(12):1849-59.

Ke, B. 1957. "The Polarographic Behavior of Alpha-Lipoic Acid.” Biochim Biophys Acta 25:650-51.

Kennis, J. T. M. and M.-L. Groot. 2007. "Ultrafast Spectroscopy of Biological Photoreceptors." Current Opinion in Structural Biology 17:623-30.

Kim, H. and M. S. Patel. 1992a. "Characterization of Two Site-Specifically Mutated Human Dihydrolipoamide Dehydrogenases (His-452-Gln and Glu-457-Gln)." Journal of Biological Chemistry 267:5128-32.

Kim, L. O. Ingram, and K. T. Shanmugam. 2007. "Construction of an Escherichia coli K-12 Mutant for Homoethanologenic Fermentation of Glucose or Xylose without Foreign Genes." Applied and Environmental Microbiology 73(6):1766-71.

Kim, L. O. Ingram, and K. T. Shanmugam. 2008. "Dihydrolipoamide Dehydrogenase Mutation Alters the NADH Sensitivity of Pyruvate Dehydrogenase Complex of Escherichia coli K-12.” Journal of Bacteriology 190(11):3851-58.

Klinman, J. P. 2009. “An Integrated Model for Enzyme Catalysis Emerges from Studies of Hydrogen Tunneling." Chemical Physics Letters 471(4-6):179-93.

Klinman, J. P. 2010. "Enzyme Dynamics: Control of Active-Site Compression." Nature Chemistry 2(11):907-9.

Korotchkina, L. G. and M. S. Patel. 2001. "Probing the Mechanism of Inactivation of Human Pyruvate Dehydrogenase by Phosphorylation of Three Sites." Journal of Biological Chemistry 276(8):5731-38.

Kottke, T., J. Heberle, D. Hehn, B. Dick, and P. Hegemann. 2003. "Phot-LOV1: Photocycle of a BlueLight Receptor Domain from the Green Alga Chlamydomonas reinhardtii." Biophysical Journal 84(February):1192-1201.

Koziol, J. 1971. "Fluorometric Analyses of Riboflavin and Its Coenzymes." Pp. 253-85 in Methods in Enzymology. McCormick, D. B. and Wright, L. D., eds.

Laemmli, U. K. 1970. "Cleavage of Structural Proteins during the Assembly of the Head of Bacteriophage T4." Nature 227:680-85. 
Ledwidge, R. et al. 2005. "NmerA, the Metal Binding Domain of Mercuric Ion Reductase, Removes Hg 2+ from Proteins, Delivers It to the Catalytic Core, and Protects Cells under GlutathioneDepleted Conditions." Biochemistry 44:11402-16.

Levitch, E. 1958. "Studies on the Nature Protein-Bound." The Journal of Biological Chemistry 232:143-58.

Lindsay, H. et al. 2000. "FAD Insertion Is Essential for Attaining the Assembly Competence of the Dihydrolipoamide Dehydrogenase (E3) Monomer from Escherichia coli." Journal of Biological Chemistry 275(47):36665-70.

Losi, A. and W. Gärtner. 2011. "Old Chromophores, New Photoactivation Paradigms, Trendy Applications: Flavins in Blue Light-Sensing Photoreceptors." Photochemistry and Photobiology 87:491-510.

Losi, A. and W. Gärtner. 2012. "The Evolution of Flavin-Binding Photoreceptors: An Ancient Chromophore Serving Trendy Blue-Light Sensors.” Annual Review of Plant Biology 63:49-72.

Losi, A, T. Kottke, and P. Hegemann. 2004. "Recording of Blue Light-Induced Energy and Volume Changes within the Wild-Type and Mutated Phot-LOV1 Domain from Chlamydomonas reinhardtii." Biophysical journal 86(2):1051-60.

Losi, A., B. Quest, and W. Gärtner. 2003. "Listening to the Blue: The Time-Resolved Thermodynamics of the Bacterial Blue-Light Receptor YtvA and Its Isolated LOV Domain." Photochemical \& photobiological sciences: Official Journal of the European Photochemistry Association and the European Society for Photobiology 2:759-66.

Lüdtke, S. et al. 2013. "Sub-Ångström-Resolution Crystallography Reveals Physical Distortions That Enhance Reactivity of a Covalent Enzymatic Intermediate." Nature chemistry 5(September):762-67.

Lyubimov, A. Y., K. Heard, H. Tang, N. S. Sampson, and A. Vrielink. 2007. "Distortion of Flavin Geometry Is Linked to Ligand Binding in Cholesterol Oxidase." Protein Science : A Publication of the Protein Society 16:2647-56.

Machado, R. S., D. P. Clark, and J. R. Guest. 1992. "Construction and Properties of Pyruvate Dehydrogenase Complexes with up to Nine Lipoyl Domains per Lipoate Acetyltransferase Chain." FEMS Microbiology Letters 79:243-48. 
Maeda-Yorita, K., G. C. Russell, J. R. Guest, V. Massey, and C. H. Williams. 1991. "Properties of Lipoamide Dehydrogenase Altered by Site-Directed Mutagenesis at a Key Residue (I184Y) in the Pyridine Nucleotide Binding Domain.” Biochemistry 30:11788-95.

Maeda-Yorita, K., G. C. Russell, J. R. Guest, V. Massey, and C. H. Williams. 1994. "Modulation of the Oxidation-Reduction Potential of the Flavin in Lipoamide Dehydrogenase from Escherichia Coli by Alteration of a Nearby Charged Residue, K53R.” Biochemistry 33:6213-20.

Mande, S. S., S. Sarfaty, M. D. Allen, R. N. Perham, and W. G. Hol. 1996. "Protein-Protein Interactions in the Pyruvate Dehydrogenase Multienzyme Complex: Dihydrolipoamide Dehydrogenase Complexed with the Binding Domain of Dihydrolipoamide Acetyltransferase." Structure 4:277-86.

Mansfield, C. W. 1960. Oxidation-Reduction Poentials Of Organic Systems. The Williams \& Wilkins Company.

Massey, V. and Q. H. Gibson. 1964. "Role Of Semiquinones In Flavoproteins Catalysis." Federation Proceedings 23:18-29.

Massey, V., Q. H. Gibson, and C. Veeger. 1960. "Intermediates in the Catalytic Action of Lipoyl Dehydrogenase (Diaphorase)." The Biochemical Journal 77(1958):341-51.

Massey, V. and G. Palmer. 1966. "On the Existence of Spectrally Distinct Classes of Flavoprotein Semiquinones. A New Method for the Quantitative Production of Flavoprotein Semiquinones." Biochemistry 5(10):3181-89.

Mattevi, A, G. Obmolova, K. H. Kalk, A. Teplyakov, and W. G. Hol. 1993. "Crystallographic Analysis of Substrate Binding and Catalysis in Dihydrolipoyl Transacetylase (E2p)." Biochemistry 32:3887-3901.

Mattevi, A, A.J. Schierbeek, and W.G. Hol. 1991. "Refined Crystal Structure of Lipoamide Dehydrogenase from Azotobacter Vinelandii at $2.2 \AA$ Resolution. A Comparison with the Structure of Glutathione Reductase.” Journal of Molecular Biology 220:975-94.

Matthews, G., P. Ballou, and C.H. Williams. 1979. "Reactions of Pig Hear Lipoamide Dehydrogenase with Pyridine Nucleotides." The Journal of Biological Chemistry 254(12):4974-81.

Matthews, G., and C.H. Williams. 1976. "Measurement of the Oxidation-Reduction Potentials for Two-Electron and Four-Electron Reduction of Lipoamide Dehydrogenase from Pig Heart." The Journal of Biological Chemistry 251(13):3956-64. 
McCoy, A. J. 2006. "Solving Structures of Protein Complexes by Molecular Replacement with Phaser." Acta Crystallographica Section D: Biological Crystallography 63:32-41.

McCoy, A. J. et al. 2007. "Phaser Crystallographic Software." Journal of Applied Crystallography 40:658-74.

McDonald, C. A., Y. Y. Liu, and B. A. Palfey. 2013. "Actin Stimulates Reduction of the MICAL-2 Monooxygenase Domain.” Biochemistry 52:6076-84.

Miller, S. M. et al. 1990. "Use of a Site-Directed Triple Mutant to Trap Intermediates: Demonstration That the Flavin C(4a)-Thiol Adduct and Reduced Flavin Are Kinetically Competent Intermediates in Mercuric Ion Reductase.” Biochemistry 29:2831-41.

Möglich, A., X. Yang, R. A. Ayers, and K. Moffat. 2010. "Structure and Function of Plant Photoreceptors." Annual Review of Plant Biology 61:21-47.

Müller, F., P. Hemmerich, A Ehrenberg, G. Palmer, and V. Massey. 1970. "The Chemical and Electronic Structure of the Neutral Flavin Radical as Revealed by Electron Spin Resonance Spectroscopy of Chemically and Isotopically Substituted Derivatives." European Journal of Biochemistry / FEBS 14:185-96.

Müller, Y. A., G. Schumacher, R. Rudolph, and G. F. Schulz. 1994. "The Refinded Structures of a Stabilized Mutant and of Wild-Type Pyruvate Oxidase from Lactobacillus plantarum.” Journal of Molecular Biology 237:315-35.

Neumann, P., A. Weidner, A. Pech, M. T. Stubbs, and K. Tittmann. 2008. "Structural Basis for Membrane Binding and Catalytic Activation of the Peripheral Membrane Enzyme Pyruvate Oxidase from Escherichia coli." Proceedings of the National Academy of Sciences of the United States of America 105(45):17390-95.

O'Donnell, M. and C. H. Williams. 1984. "Reconstitution of Escherichia coli Thioredoxin Reductase with 1-DeazaFAD.” The Journal of Biological Chemistry 259(4):2243-51.

Palfey, B. A. and R. Fagan. 2010. "Flavin-Dependent Enzymes.” Pp. 38-42 in Comprehensive Natural Products II, edited by L. Mader and L. Hung-Wen.

Patel, M. S. and L. G. Korotchkina. 2001. "Regulation of Mammalian Pyruvate Dehydrogenase Complex by Phosphorylation: Complexity of Multiple Phosphorylation Sites and Kinases.” Experimental \& Molecular Medicine 33(4):191-97.

Patel, M. S. and T. E. Roche. 1990. "Molecular Biology and Biochemistry of Pyruvate Dehydrogenase Complexes." The FASEB Journal 4:3224-33. 
Perczel, A. and G.D. Fasman. 1992. "Quantitative Analysis of Cyclic Beta-Turn Models." Protein Science: A Publication of the Protein Society 1:378-95.

Perham, R. N. 2000. "Swinging Arms And Swinging Domains In Multfunctional Enzymes : Catalytic Machines for Multistep Reactions.” Annu. Rev. Biochem. 69:961-1004.

Perham, R. N. and P.A. Reche. 1998. "Swinging Arms in Multifunctional Enzymes and the Specificity of Post-Translational Modification." Biochem Soc Trans 26:299-303.

Pfeifer, A. et al. 2009. "Time-Resolved Fourier Transform Infrared Study on Photoadduct Formation and Secondary Structural Changes within the Phototropin LOV Domain.” Biophysical Journal 96(4):1462-70.

Purcell, E. B., C. A. McDonald, B. A. Palfey, and S. Crosson. 2010. "An Analysis of the Solution Structure and Signaling Mechanism of LovK, a Sensor Histidine Kinase Integrating Light and Redox Signals." Biochemistry 49:6761-70.

Raffelberg, S., M. Mansurova, W. Gärtner, and Aba Losi. 2011. "Modulation of the Photocycle of a LOV Domain Photoreceptor by the Hydrogen-Bonding Network." Journal of the American Chemical Society 133(5):5346-56.

Reed, J. K. 1973. "Studies on the Kinetic Mechanism of Lipoamide Dehydrogenase from Rat Liver Mitochondria." The Journal of Biological Chemistry 248(11):4834-39.

Reed, L. J. 2001. “A Trail of Research from Lipoic Acid to Alpha-Keto Acid Dehydrogenase Complexes." The Journal of Biological Chemistry 276(42):38329-36.

Reed, Lester J. 1974. “Multienzyme Complexes.” Accounts of Chemical Research 7:40-46.

Reed, Lester J., Masahiko Koike, and P. C. Shah. 1960. "Alpha-Keto Acid Dehydrogenation Complexes - Purification and Propertie of Dihydrolipoic Dehydrogenase of Escherichia coli." The Journal of Biological Chemistry 235(7):1939-43.

Rizzo, Carmelo J. 2001. "Further Computational Studies on the Conformation of 1,5Dihydrolumiflavin.” Antioxidants \& Redox Signaling 3(5):737-46.

Rodríguez-Otero, J., E. Martínez-Núñez, A. Peña-Gallego, and S. A. Vázquez. 2002. "The Role of Aromaticity in the Planarity of Lumiflavin." Journal of Organic Chemistry 67(10):6347-52.

Rosentel, J. K., F. Healy, J.A. Maupin-Furlow, J. Ho Lee, and K. T. Shanmugam. 1995. "Molybdate and Regulation of Mod (molybdate Transport), fdhF, and Hyc (formate Hydrogenlyase) Operons in Escherichia coli." Journal of Bacteriology 177(17):4857-64. 
Sahlman, L., A.M. Lambeir, and S. Lindskog. 1986. "Rapid-Scan Stopped-Flow Studies of the pH Dependence of the Reaction between Mercuric Reductase and NADPH." European Journal of Biochemistry / FEBS 156:479-88.

Sahlman, L. and C.H. Williams. 1989. "Lipoamide Dehydrogenase from Escherichia coli Steady-State Kinetics of the Physiological Reaction.” Journal of Biological Chemistry 264(14):8039-45.

Sattar, A., J. M. deMan, and J. C. Alexander. 1977. "Light-Induced Degradation of Vitamins I. Kinetic Studies on Riboflavin Decomposition in Solution." Canadian Institute of Food Science and Technology Journal 10(1):61-64.

Schulz, George E. and Emil F. Pai. 1983. "The Catalytic Mechanism of Glutathione Reductase as Derived from X-Ray Diffraction Analyses of Reaction Intermediates.” The Journal of Biological Chemistry 258(24):1752-57.

Schuman Jorns, M., G. Schöllnhammer, and P. Hemmerich. 1975. "Intramolecular Addition of the Riboflavin Side Chain.” FEBS Journal 57(1):35-48.

Searls, R., J. Peters, and D. Sanadi. 1961. "Alpha-Ketoglutaric Dehydrogenase - On the Mechanism of Dihydrolipoyl Dehydrogenase Reaction.” The Journal of Biological Chemistry 236(8):2317-22.

Seifert, F. et al. 2007. "Phosphorylation of Serine 264 Impedes Active Site Accessibility in the E1 Component of the Human Pyruvate Dehydrogenase Multienzyme Complex." Biochemistry 46:6277-87.

Seifert, F. 2010. "Katalyse und Regulation Der E1-Komponente Des Humanen PyruvatDehydrogenase-Komplexes Auf Molekularer Ebene.” Martin-Luther-Universität HalleWittenberg.

Shen, L. C., L. Fall, G. M. Walton, and D. E. Atkinson. 1968. "Interaction between Energy Charge and Metabolite Modulation in the Regulation of Enzymes of Amphibolic Sequences. Phosphofructokinase and Pyruvate Dehydrogenase." Biochemistry 7:4041-45.

Sheraz, M. A., S. H. Kazi, S. Ahmed, T. Mirza, et al. 2014. "Effect of Phosphate Buffer on the Complexation and Photochemical Interaction of Riboflavin and Caffeine in Aqueous Solution: A Kinetic Study.” Journal of Photochemistry and Photobiology B: Biology 273:17-22.

Sheraz, M. A., S. H. Kazi, S. Ahmed, Z. Anwar, and I. Ahmad. 2014. "Photo, Thermal and Chemical Degradation of Riboflavin.” Beilstein Journal of Organic Chemistry 1999-2012. 
Shoyama, Y. et al. 2012. "Structure and Function of $\Delta 1$-Tetrahydrocannabinolic Acid (THCA) Synthase, the Enzyme Controlling the Psychoactivity of Cannabis sativa." Journal of Molecular Biology 423(1):96-105.

Smith, M. W. and F. C. Neidhardt. 1983. "2-Oxoacid Dehydrogenase Complexes of Escherichia coli: Cellular Amounts and Patterns of Synthesis.” Journal of Bacteriology 156(1):81-88.

Snoep, J.L. et al. 1993. "Differences in Sensitivity to NADH of Purified Pyruvate Dehydrogenase Complexes of Enterococcus faecalis, Lactococcus lactis, Azotobacter vinelandii and Escherichia coli: Implications for Their Activity in Vivo." FEMS Microbiology Letters 114(3):279-83.

Talfournier, F. et al. 2001a. " $\alpha \mathrm{Arg}-237$ in Methylophilus methylotrophus (sp. $\mathrm{W}_{3} \mathrm{~A}_{1}$ ) ElectronTransferring Flavoprotein Affords $\sim 200$-Millivolt Stabilization of the FAD Anionic Semiquinone and a Kinetic Block on Full Reduction to the Dihydroquinone." Journal of Biological Chemistry 276:20190-96.

Thorpe, C. and C. H. Williams. 1976a. "Differential Reactivity of the Two Active Site Cysteine Residues Generated on Reduction of Pig Heart Lipoamide Dehydrogenase." Journal of Biological Chemistry 251:3553-57.

Thorpe, C. and C. H. Williams. 1976b. "Spectral Evidence for a Flavin Adduct in a Monoalkylated Derivative of Pig Heart Lipoamide Dehydrogenase.” Journal of Biological Chemistry 251:772628.

Thorpe, C. and C. H. Williams. 1981. "Lipoamide Dehydrogenase from Pig Heart. Pyridine Nucleotide Induced Changes in Monoalkylated Two-Electron Reduced Enzyme." Biochemistry 20:1507-13.

Tittmann, K. 2014. "Bioorganic Chemistry Sweet Siblings with Different Faces : The Mechanisms of FBP and F6P Aldolase, Transaldolase, Transketolase and Phosphoketolase Revisited in Light of Recent Structural Data." Bioorganic Chemistry 57:263-80.

Unden, G. and J. Bongaerts. 1997. "Alternative Respiratory Pathways of Escherichia Coli: Energetics and Transcriptional Regulation in Response to Electron Acceptors." Biochimica et Biophysica Acta - Bioenergetics 1320:217-34.

Walsh, J. D. and A. F. Miller. 2003. "Flavin Reduction Potential Tuning by Substitution and Bending." Journal of Molecular Structure: THEOCHEM 623:185-95. 
Wang, J. et al. 2014. "Structure and Function of the Catalytic Domain of the Dihydrolipoyl Acetyltransferase Component in Escherichia coli Pyruvate Dehydrogenase Complex." Journal of Biological Chemistry 289:15215-30.

Wei, W., H. Li, N. Nemeria, and F. Jordan. 2003. "Expression and Purification of the Dihydrolipoamide Acetyltransferase and Dihydrolipoamide Dehydrogenase Subunits of the Escherichia coli Pyruvate Dehydrogenase Multienzyme Complex: A Mass Spectrometric Assay for Reductive Acetylation of Dihydrolipoamide." Protein Expression and Purification 28:14050.

Whitby, L.G. 1953. “A New Method for Preparing Flavin-Adenine Dinucleotide.” The Biochemical Journal 54(1946):437-42.

Wilkinson, K. D. and C.H. Williams. 1979. "Evidence for Multiple Electronic Forms of TwoElectron-Reduced Lipoamide Dehydrogenase from Escherichia coli." Journal of Biological Chemistry 254:852-62.

Wilkinson, K.D. and C.H. Williams. 1981. "NADH Inhibition and NAD Activation of Escherichia coli Lipoamide Dehydrogenase Catalyzing the NADH-Lipoamide Reaction." Journal of Biological Chemistry 256(5):2307-14.

Williams, C. H. 1965. "Studies on Lipoyl Dehydrogenase from Escherichia coli." Journal of Biological Chemistry 240(12):4793-4800.

Williams, C.H., G. Zanetti, L. D. Arscott, and J. K. McAllister. 1967. "Lipoamide Dehydrogenase, Glutathione Reductase, Thioredoxin Reductase, and Thioreoxin." The Journal of Biological Chemistry 242(22):5226-31.

Winn, M. D. et al. 2011. "Overview of the CCP4 Suite and Current Developments." Acta Crystallographica Section D: Biological Crystallography 67:235-42.

Zheng, Y. J. and R. L. Ornstein. 1996. "A Theoretical Study of the Structures of Flavin in Different Oxidation and Protonation States." Journal of the American Chemical Society 118(16):9402-8.

Zoltowski, B. D. and K. H. Gardner. 2011. "Tripping the Light Fantastic: Blue-Light Photoreceptors as Examples of Environmentally Modulated Protein-Protein Interactions." Biochemistry 50:4-16.

Zoltowski, B. D., B. Vaccaro, and B. R. Crane. 2009. "Mechanism-Based Tuning of a LOV Domain Photoreceptor." Nature Chemical Biology 5(11):827-34. 


\section{Curriculum Vitae}

\section{Michael Tietzel}

Georg-August-University Goettingen

Department of Molecular Enzymology

Justus-von-Liebig Weg 11

37077 Goettingen, Germany

Phone: +495513914000

e-mail: mtietze1@gwdg.de

Date of Birth: $\quad$ March $7^{\text {th }} 1986$

Place of Birth: Holzminden

Nationality: German

\section{Education}

1998-05/2005 Campe Gymnasium Holzminden

10/2005-09/2006 Military service

10/2006-09/2009 BSc Program Biology, Georg-August-Universität Göttingen

$09 / 2009$

Bachelor of Science

Department of Molecular Structural Biology: „Biochemische und kristallografische Charakterisierung des TRBP-Dicer-Komplexes“

10/2009-11/2011 MSc/PhD Program Microbiology and Biochemistry, Georg-August-Universität Göttingen

11/2011 Master of Science, Georg-August-Universität Göttingen

Department of Molecular Enzymology: „Biochemical analyses of proteinprotein interactions in the E.coli pyruvate dehydrogenase complex“

02/2012-07/2015 GGNB doctoral program Biomolecules: Structure - Function -Dynamics, Georg-August-Universität Göttingen, Department of Molecular Enzymology 